Can modern food retailers improve diets and nutrition in urban Africa?

Empirical evidence from Zambia

Dissertation

to obtain the Ph.D. degree

in the International Ph.D. Program for Agricultural Sciences in Goettingen (IPAG)

at the Faculty of Agricultural Sciences,

Georg-August-University Göttingen, Germany

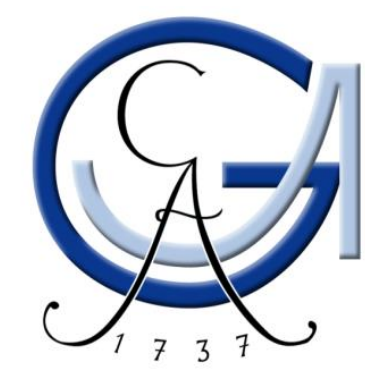

presented by

Makaiko Gonapanyanja Khonje

born in Mzimba (Lusangazi), Malawi

Göttingen, March 2020 
D7

1. Name of supervisor: Prof. Dr. Matin Qaim

2. Name of co-supervisor: Prof. Stephan Klasen, $\mathrm{PhD}$

3. Name of co-supervisor: Prof. Dr. Sebastian Vollmer

Date of dissertation: 19.05 .2020 


\section{Summary}

In many developing countries, food environments are changing rapidly, with modern food retailers - such as supermarkets and convenience stores - increasingly replacing or complementing traditional food retailers. In comparison to traditional food markets and shops, modern retailers often sell a different range of products, at different prices and packaging sizes, and in different shopping atmospheres, thus affecting people's food environments and potentially also their food choices, diets, and nutrition. Understanding the dietary and nutrition effects of a modernizing retail sector is important, as many countries in Africa suffer from a double or even a triple burden of malnutrition - i.e., the coexistence of undernutrition, micronutrient deficiencies, and overweight or obesity within the same communities, households, and even individuals - with serious negative health consequences. Recent research suggests that access to and affordability of healthy diets remain formidable challenges in many developing countries. However, empirical studies analyzing the effects of modern retailers on consumer diets, dietary costs, and nutrition remain scant.

A few recent studies showed that the growth of modern retailers, especially supermarkets, in developing countries contributes to higher consumption of ultra-processed foods and rising rates of overweight and obesity among adults. For children, research on the links between modern retailers and child nutrition is still limited; the few existing studies found mixed results. In any case, the available evidence suggests that modern retailers may have different effects on dietary quality and nutrition among adults and children. One major drawback of existing studies is that they analyzed individual-level anthropometric data, yet without having individual-level dietary data to explain some of the underlying mechanisms. Therefore, none of the previous studies analyzed the effects of modern retailers - such as supermarkets - on dietary quality (i.e., nutrient intake) with individual-level dietary data to account for intrahousehold food distribution. Another drawback is that previous studies mostly focused on supermarkets, without accounting for the fact that other types of modern retailers - such as convenience stores and fast-food restaurants - are also gaining in importance as sources of food in urban Africa. Finally, the role of supermarkets and other modern retailers on the affordability of nutritious diets was not analyzed in any of the existing studies.

We make several contributions to the existing literature by addressing the highlighted research gaps in the three papers of this dissertation. In the first paper, we examine the relationships between consumers' socioeconomic status, use of different modern and traditional retailers, and dietary patterns. The analysis uses household survey data from urban Zambia. We 
surveyed a total of 475 urban households in 2018. Results show that two-thirds of the households use modern and traditional retailers simultaneously, but that richer households are more likely than poorer ones to use supermarkets and hypermarkets. Use of modern retailers is positively associated with higher consumption of ultra-processed foods, after also controlling for income and other socioeconomic factors. However, the use of traditional stores and kiosks is also positively associated with the consumption of ultra-processed foods, suggesting that modern retailers are not the only drivers of dietary transitions.

In the second paper, we provide the first empirical study that analyzes effects of modern retailers on dietary quality and nutrition with individual-level food-intake/dietary and anthropometric data in a developing country. We collected data from 475 randomly selected households that use modern retailers at different intensities in Lusaka, Zambia. In these households, individual-level anthropometric and food-intake data were collected from 930 adults and 499 children. The data are analyzed with control function regression models to address potential endogeneity problems associated with food purchases made in modern retailers. We find that use of modern retailers is positively associated with overweight in adults, but not in children. For children, we find a positive effect on body height, also after controlling for income and other relevant factors. Use of modern retailers increases dietary diversity, calorie, protein, and micronutrient intakes among both adults and children. Effects on protein and micronutrient intakes are channeled primarily through higher consumption of meat and dairy products.

In the third paper, we analyze effects of using supermarkets on the affordability of recommended nutritious diets and dietary quality. We use individual-level food-intake data and food price data from our household survey conducted in Lusaka, Zambia, and control function regression models to account for the likely endogeneity of supermarket food purchases. We find that the cost of a recommended nutritious diet is US\$1.22 per day, of which the largest share is the cost of starchy staples (68\%), followed by fruits (11\%), and meat, eggs, and fish (9\%). However, this diet is not affordable to $41 \%$ of low-income group. Meat, fish, and dairy products are more expensive in supermarkets than in traditional retailers. Nevertheless, buying food in supermarkets increases dietary diversity and intake of nutritious diets, with varying effect sizes among demographic cohorts: men, women, boys, and girls. The positive effects of supermarkets on dietary quality largely come from animal source foods. 
We draw several conclusions and policy implications from the three papers in this dissertation. The findings underline that the growth of modern food retailers in developing countries influences people's diets and nutrition; the effects can be both positive and negative. The positive effects on child nutrition and dietary quality of both children and adults imply that further modernization of food environments should be promoted. However, due to higher quality food products and safety standards, modern retailers - such as supermarkets - offer higher prices for meat, fish, and dairy products than traditional retailers. Thus, the results suggest a need to shift food policy from focusing on energy-dense foods to affordable nutritious foods. Modern retailers could be one of the platforms to make nutritious foods i.e., meat, fish, eggs, milk, and other dairy products - more affordable especially among poor households. Lower prices could come from improvements in local production, higher efficiency in procurements, marketing and trade, and infrastructure developments especially cooling facilities and warehouses.

On the other hand, the increasing effect of modern retailers on the consumption of ultraprocessed foods and adult overweight is undesirable and calls for certain policy regulations. Possible policy interventions include regulation of advertisement and promotional campaigns for unhealthy foods, regulation of health labels and portion/packaging sizes, taxes on ultraprocessed foods and beverages with high contents of fats, added sugars and salts, among others. While the results cannot be generalized, effects may be similar also in other parts of Africa. Nevertheless, further research is needed to better understand the diet and nutrition effects of changing food environments in different geographical and socioeconomic contexts, and also focusing on long-run dynamics. 


\section{Acknowledgements}

First, I thank the Almighty God for giving me strength, good health, and the ability to successfully complete my Ph.D. studies. I also express my sincere gratitude to my supervisor Prof. Dr. Matin Qaim for offering me a doctoral research position in his research group, and for his precious academic guidance throughout my studies, and being a great mentor! His feedbacks were insightful and timely; making the burden of Ph.D. studies to be relatively lighter. My special thanks also go to Prof. Stephan Klasen and Prof. Dr. Sebastain Vollmer for co-supervising and serving on my dissertation examination committee.

I am grateful to Dr. Olivier Ecker, my counterpart at the International Food Policy Research Institute (IFPRI), who hosted me during my research stay at IFPRI in Washington, DC, and who closely cooperated with me especially on the second paper. I am very grateful to Dr. Melanie Grossie and Katrin Pape from the coordination office of GlobalFood Research Training Group (RTG 1666), and Ilona Hoepfner for their administrative assistance. I express my special thanks to Dr. Kibrom Sibhatu, Dr. Esther Gehrke, Dr. Bethelhem Legesse Debela, Dr. Dennis Ochieng, Dr. Haruna Sekabira, Dr. Zewdu Ayalew Abro, Dr. Sylvester O. Ogutu, and Dr. Rosina Wanyama for their excellent friendship. To all my fellow doctoral students from RTG 1666 and at the Chair of International Food Economics and Rural Development, your friendship and collaborations made my stay in Goettingen more enjoyable.

This research was financially supported by the German Research Foundation (DFG) as part of the RTG 1666. This financial support is gratefully acknowledged. Appreciations are due to Ms. Patricia Sakala and Mr. Boniface Kanjere from the National Food and Nutrition Commission of Zambia for providing height boards and weighing scales. Special thanks to our five enumerators (Eric Chikwalila, Eugine Kaango, Samba Manjolo, Brian Mpande, and Kelvin Sinyinza) and our field supervisor (Mully Phiri) who made the fieldwork possible. Thanks to all the respondents who voluntarily spared their time to participate in the survey.

Finally, I wish to express my deepest gratitude to my dear wife Maggie Musukwa Khonje, for her tireless support and encouragement throughout my Ph.D. studies, and for taking good care of our two sons - Jeremiah and Joshua - in my long absence. I also extend my special thanks to my parents, Mr. Stocker Gonapanyanja Khonje and Mrs. Selina Chihana Khonje for their love, prayers, encouragement, guidance, and inspiring me to value education and working hard in my undertakings. To my brothers and sisters, many thanks for your moral support and love. This dissertation is dedicated to everyone in my family, you are all special! 


\section{Table of contexts}

Summary

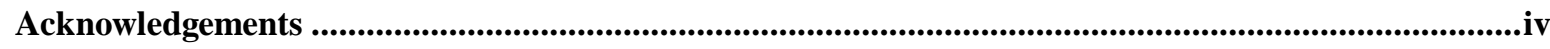

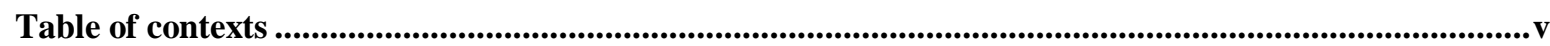

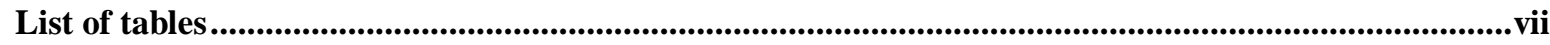

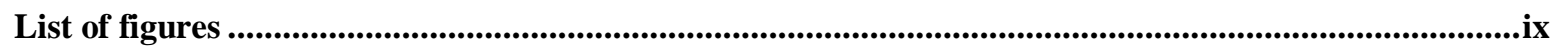

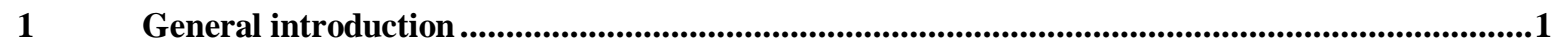

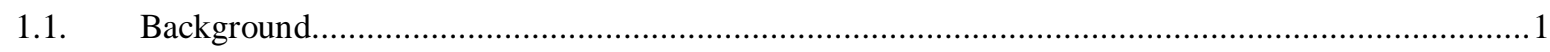

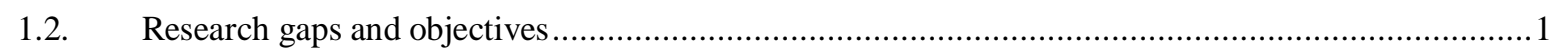

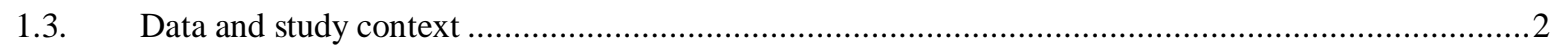

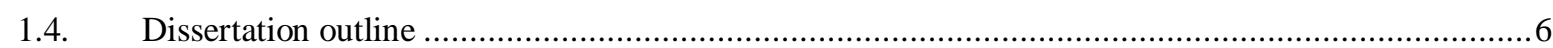

$2 \quad$ Modernization of African food retailing and (un)healthy food consumption ..............................7

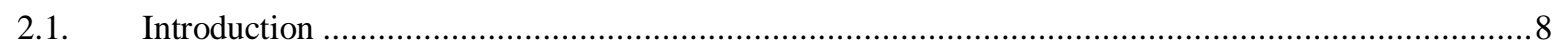

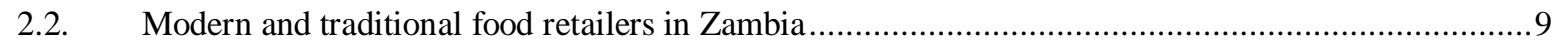

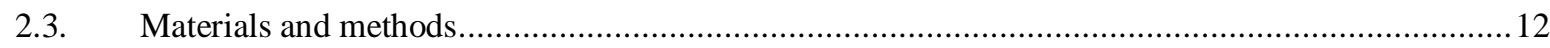

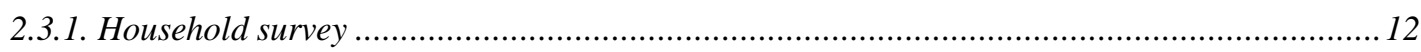

2.3.2. Measurement of key variables ......................................................................... 12

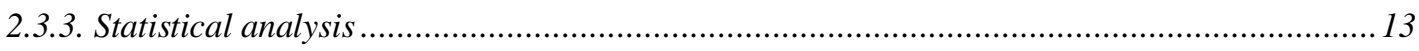

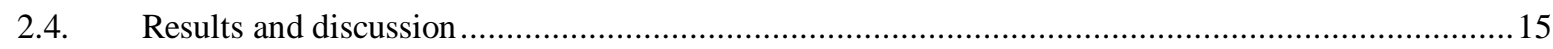

2.4.1. Household socioeconomic characteristics ............................................................... 15

2.4.2. Role of modern and traditional retailers...................................................................... 17

2.4.3. Factors influencing the use of modern retailers ............................................................ 19

2.4.4. Associations between retailers and food processing levels ..............................................22

2.4.5. Associations between retailers and food groups...........................................................24

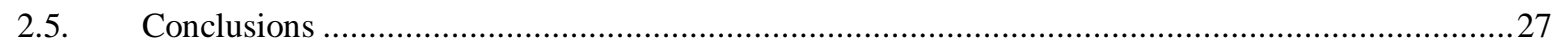

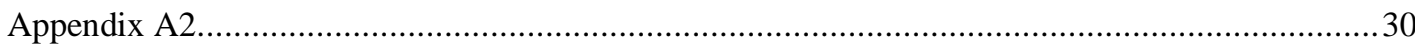

$3 \quad$ Effects of modern food retailers on adult and child diets and nutrition....................................40

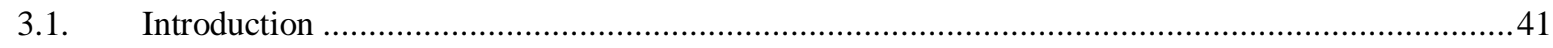

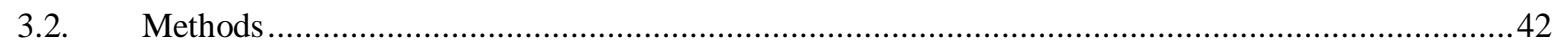

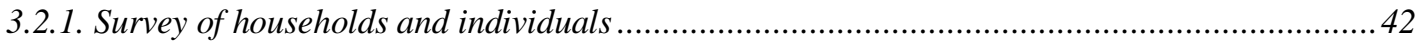

3.2.2. Measuring nutrition and dietary quality .............................................................. 43

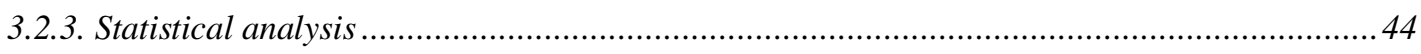

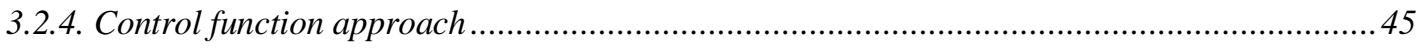


3.3. Results . .47

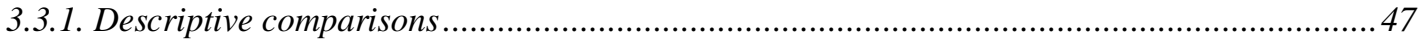

3.3.2. Effects of modern retailers on nutrition status ................................................................ 49

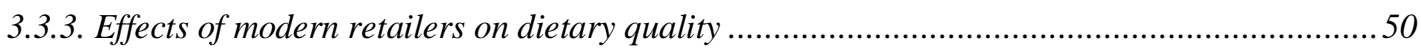

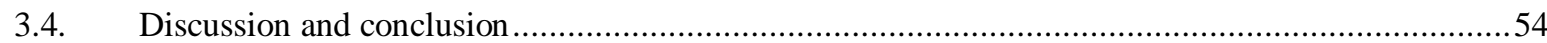

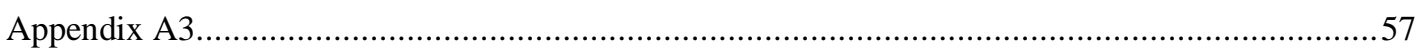

4 Supermarkets and affordability of nutritious diets: Evidence from urban Zambia .................73

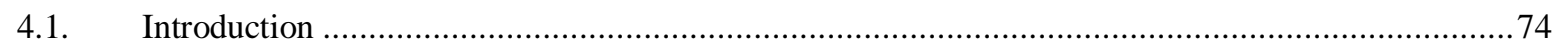

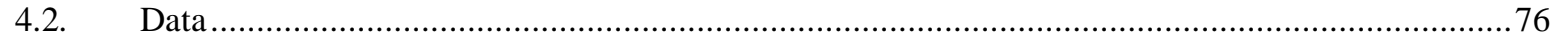

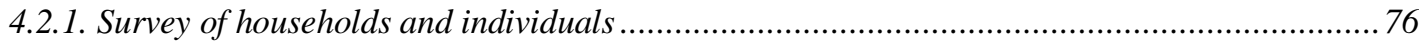

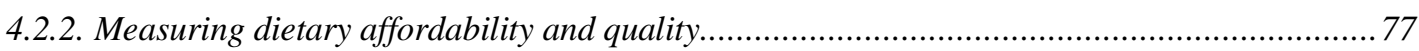

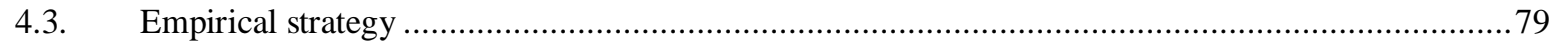

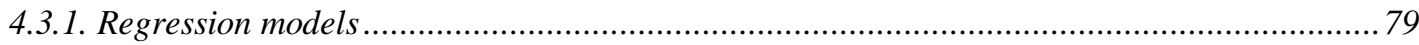

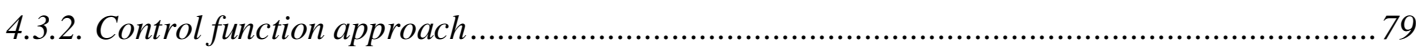

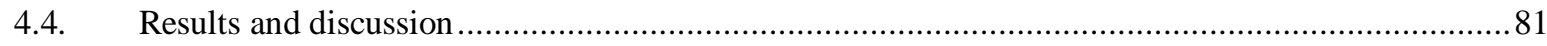

4.4.1. Cost of recommended nutritious diets ........................................................................ 81

4.4.2. Role of supermarkets on affordability of nutritious diets ...................................................83

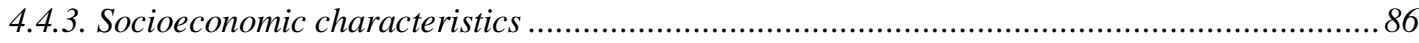

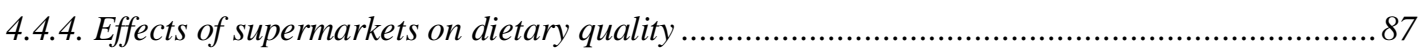

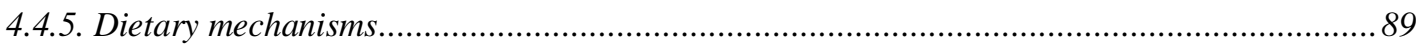

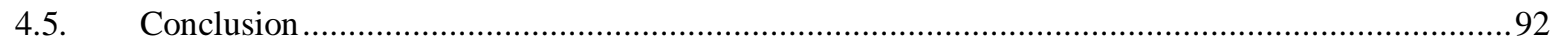

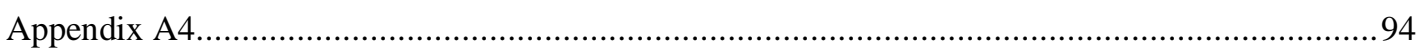

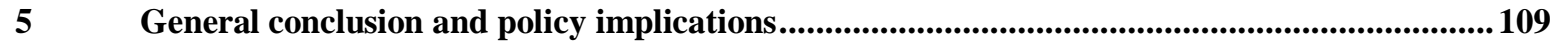

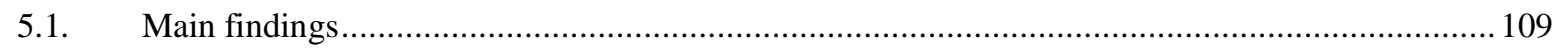

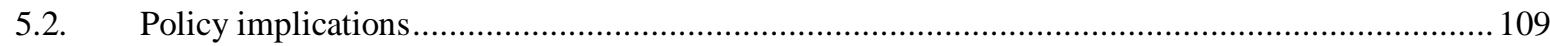

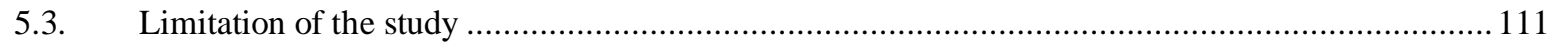

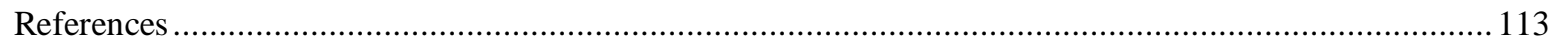

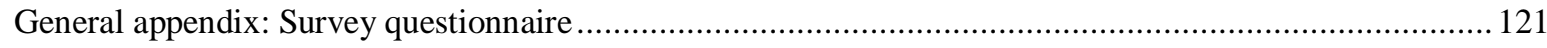




\section{List of tables}

Table 1.1: List of main shopping malls with modern food retailers in Lusaka City......

Table 2.1: Key characteristics of different food retailers in Lusaka City, Zambia

Table 2.2: Socioeconomic characteristics and food consumption patterns

Table 2.3: Proportion of households using different modern and traditional retailers

Table 2.4: Factors influencing the use of different food retailers (Multivariate Probit Model)

Table 2.5: Associations between the use of different retailers and food processing levels

Table 2.6: Associations between the use of different retailers and the consumption of selected food groups ...... 25

Table A2.1: Food processing levels by food groups and items 30

Table A2.2: Additional descriptive statistics

Table A2.3: Correlation matrix from Multivariate Probit Model.

Table A2.4: Associations between the use of retailers and food processing levels (full model results for Table 2.5)

Table A2.5: Associations between supermarket use and food processing levels (Seemingly unrelated regressions)

Table A2.6: Associations between the use of different retailers and food processing levels (absolute expenditures)

Table A2.7: Associations between the use of different retailers and food processing levels (by poverty status).. 36

Table A2.8: Associations between the use of different retailers and the consumption of food groups (full model results for Table 2.6, supermarkets only).

Table A2.9: Associations between the use of different retailers and the consumption of food groups (full model results for Table 2.6, all retailers).

Table A2.10: Associations between the use of different retailers and the consumption of food groups (in value terms)

Table 3.1: Per capita food intake of adults and children using and not using modern retailers

Table 3.2: Nutrition and dietary indicators for adults and children using and not using modern retailers. 48

Table 3.3: Effects of using modern retailers on nutrition status. 49

Table A3.1: Distribution of sampled individuals in Lusaka City. 57

Table A3.2: Food groups and components used for construction of dietary quality indicators ........................58

Table A3.3: Estimated average requirements of calories and nutrients by sex and age cohort ........................59

Table A3.4: First-stage estimation results on food purchases in modern retailers .................................... 60

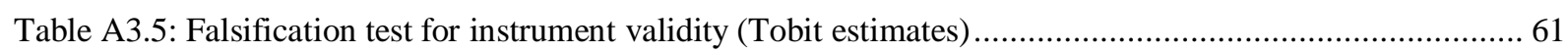

Table A3.6: Additional descriptive statistics for users and non-users of modern retailers ............................62 62

Table A3.7: Effects of modern retailers on nutritional status (full model results for Table 3.3) .....................63

Table A3.8: Effects of modern retailers on adult dietary diversity (full model results for Figure 3.1) ..............64

Table A3.9: Effects of modern retailers on child dietary diversity (full model results for Figure 3.1) ..............65

Table A3.10: Effects of modern retailers on adult calorie and nutrient intakes (full model results for Figure 3.2)

Table A3.11: Effects of modern retailers on child calorie and nutrient intakes (full model results for Figure 3.2) 
Table A3.12: Effects of modern retailers on adult micronutrient adequacy ratios

Table A3.13: Effects of modern retailers on child micronutrient adequacy ratios ....................................69

Table A3.14: Effects of supermarkets on child and adult calorie and nutrient intakes.................................. 70

Table A3.15: Effects of modern retailers on calorie and nutrient intakes of individuals from poor households (model results for Figure 3.3)

Table A3.16: Effects of modern retailers on calorie and nutrient intakes disaggregated by sex ...................... 72

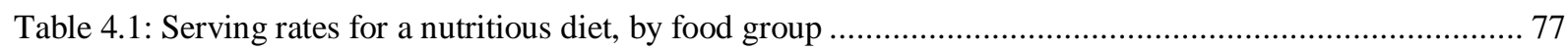

Table 4.2: Cost of recommended nutritious diets by supermarket users, demographic cohorts and income terciles 82

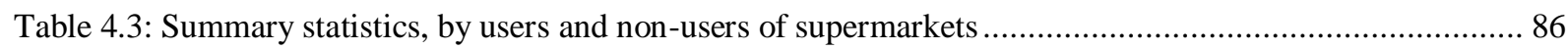

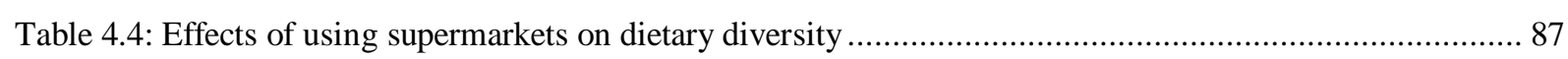

Table 4.5: Effects of using supermarkets on dietary intake ................................................................... 90

Table A4.1: The distribution of the sampled individuals in Lusaka City, Zambia.......................................94

Table A4.2: Descriptive statistics for food prices per gram in Lusaka City, Zambia (mid-2018).....................95

Table A4.3: First stage regression results on supermarket food purchases (GLM estimates) .........................97

Table A4.4: Falsification test for instrument validity (Poisson estimates) ............................................. 98

Table A4.5: Descriptive statistics for food prices (ZMW/gram), by food groups, users and non-users of

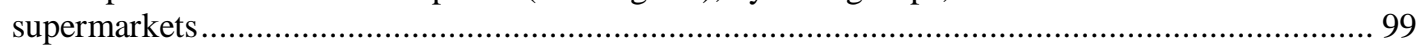

Table A4.6: Summary statistics on cost of recommended nutritious diets, by users and non-users of

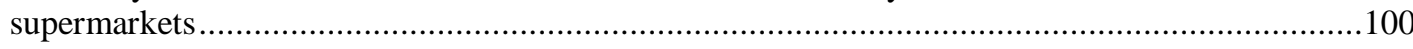

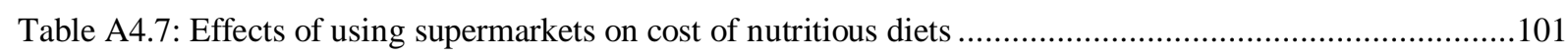

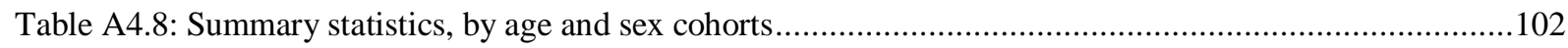

Table A4.9: Effects of using supermarkets on dietary diversity (full model results for panel A in Table 4.4) ....103

Table A4.10: Effects of using supermarkets on dietary diversity (full model results for panel B in Table 4.4) ..104

Table A4.11: Heterogeneous effects of using supermarkets on dietary diversity, by income terciles (full model

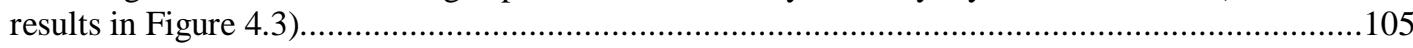

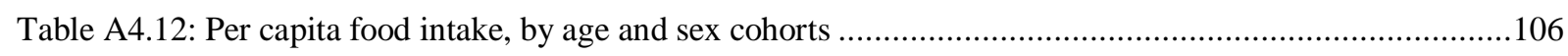

Table A4.13: Effects of using supermarkets on dietary intake (full model results in Table 4.5) .....................107

Table A4.14: Effects of using supermarkets on dietary intake: Robustness check ..................................108 


\section{List of figures}

Figure 1.1: Map of Lusaka City with sampled compounds/sections and households................................... 5

Figure 2.1: Frequency of use of modern and traditional retailers in Lusaka City ..................................... 17

Figure 2.2: Household food expenditure shares spent in different retail outlets in Lusaka City ...................... 18

Figure 3.1: Effects of using modern retailers on dietary diversity ................................................ 50

Figure 3.2: Effects of using modern retailers on calorie and nutrient intakes ............................................. 52

Figure 3.3: Effects of using modern retailers on calorie and nutrient intakes among poor households.............. 53

Figure 4.1: Cost of recommended nutritious diets, by users and non-users of supermarkets ........................ 84

Figure 4.2: Reasons for buying food in modern supermarkets ....................................................... 84

Figure 4.3: Heterogeneous effects of using supermarkets on dietary diversity, by income terciles .................. 89

Figure 4.4: Per capita food intake, by users and non-users of supermarkets ..........................................90 


\section{General introduction}

\subsection{Background}

Hunger and micronutrient malnutrition are global problems with serious negative health implications (Ruel et al., 2017; FAO et al., 2019; Swinburn et al., 2019). In many developing countries, undernutrition and micronutrient deficiencies typically coexist with rising rates of overweight and obesity within the same communities, households, and even individuals (Development Initiatives, 2018; FAO et al., 2019; Fongar et al., 2019; Harris et al., 2019; Hawkes et al., 2020; Popkin et al., 2020). Globally, it is estimated that 1.9 billion people are overweight or obese, and 600 million people have diabetes (IFPRI, 2017; Popkin, 2017). At least 2.8 million people die each year due to overweight or obesity (WHO, 2010). It is also estimated that over 800 million people are still chronically hungry, and at least 2 billion people suffer from micronutrient deficiencies worldwide (IFPRI, 2017). Approximately 45\% of all child deaths are linked to undernutrition (Black et al., 2013). Food environments, defined as the physical, economic, and sociocultural context in which consumers acquire their food, can influence dietary choices, dietary quality, and nutrition (Popkin, 2014; Popkin, 2017; Qaim, 2017; Hawkes et al., 2020).

In many developing countries, food environments are changing rapidly, with modern food retailers - such as supermarkets and convenience stores - increasingly replacing or complementing traditional food retailers (Tschirley et al., 2015; Ziba and Phiri, 2017; Lu and Reardon, 2018). Unlike traditional food markets and shops, modern retailers often sell a different range of products, at different prices and packaging sizes, and in different shopping atmospheres (Asfaw, 2008; Hawkes, 2008; Reardon and Timmer, 2014; IFPRI, 2017). Due to higher efficiency and economies-of-scale, modern retailers may contribute to a larger variety of food products becoming available and affordable for many population segments (Hawkes, 2008; Tessier et al., 2008; Popkin, 2014; Qaim, 2017). For instance, most urban consumers can access both unhealthy foods - e.g., ultra-processed foods which are often rich in fat, sugar, and salt, but poor in micronutrients - and nutritious foods from supermarkets (Asfaw, 2008; Reardon and Timmer, 2014; Rischke et al., 2015; Rupa et al., 2019; Debela et al., 2020). Recent research suggests that healthy diets are not affordable to more than 1.58 billion people (21\% of the world's population) worldwide, of which $72 \%$ are in South Asia and subSaharan Africa (Hirvonen et al., 2020). Changing food environments, especially in urban areas, may influence consumers' food choices, dietary costs, and nutrition. However, 
empirical studies analyzing the effects of modern retailers on food consumption, the affordability of recommended diets, and nutrition are relatively scant.

A few recent studies with data from Africa, Asia, and Latin America showed that the growth of modern retailers, especially supermarkets, contributes to higher consumption of highly processed foods and rising rates of overweight and obesity among adults. For instance, studies in Guatemala, Thailand, and Kenya showed that purchasing food in supermarkets leads to higher consumption of processed and highly-processed foods (Asfaw, 2008; Kelly et al., 2014; Rischke et al., 2015). In Guatemala and Kenya, supermarket food purchases were also shown to contribute to rising body mass index (BMI) and increased prevalence rates of overweight, obesity, and related chronic diseases among adults (Asfaw, 2008; Kimenju et al., 2015; Demmler et al., 2017; Demmler et al., 2018). For children, very few studies have analyzed effects of modern retailers on nutrition, and those that did found mixed results (Umberger et al., 2015; Debela et al., 2020).

\subsection{Research gaps and objectives}

While the existing research provides important evidence on the effects of modern retailers on diets and nutrition, several limitations exist. First, especially in Africa, relatively little is known about what type of consumers actually use modern supermarkets and to what extent. Another drawback is that most previous studies mostly focused on supermarkets, without accounting for the fact that other types of modern retailers - such as convenience stores and fast-food restaurants - are also gaining in importance as sources of food in urban Africa (Tschirley et al., 2015; Lu and Reardon, 2018). Moreover, focusing only on supermarkets may be misleading, as most consumers also obtain their food from various traditional retailers (Berger and van Helvoirt, 2018; Lu and Reardon, 2018; Vetter et al., 2019).

Second, one important shortcoming of all existing studies is that they did not collect individual-level food-intake/dietary data. Individual-level dietary data are important to analyze effects of modern retailers on dietary quality and better understand the mechanisms behind the nutrition impacts. Most existing studies (e.g., Asfaw, 2008; Rischke et al., 2015; Rupa et al., 2019; Debela et al., 2020) collected food consumption data at the household level, suggesting that purchasing food in supermarkets can lead to higher household dietary diversity in some situations. But household-level data neglect intra-household food distribution and are therefore not necessarily good proxies of individual-level dietary quality and micronutrient intakes. Hence, none of the available studies has analyzed effects of 
modern retailers on nutrition and dietary quality (i.e., nutrient intake) with individual-level anthropometric and food-intake data to account for intra-household food distribution in a developing country.

Third, none of the previous studies on retail modernization has analyzed effects of modern retailers on the affordability of recommended nutritious diets. Moreover, most previous studies analyzed the cost of nutritious diets only for particular target groups, such as women (e.g., Masters et al., 2018; Alemu et al., 2019; Hirvonen et al., 2020) or children (Headey et al., 2019). Yet, inequalities in dietary affordability could exist among various household members. More importantly, available studies analyzed the cost of nutritious diets using food price data from the World Bank's International Comparison Program, where only standardized food items are included (e.g., Alemu et al., 2019; Hirvonen et al., 2020). Hence, some of country-specific nutritious foods (e.g., local insects, fish, fruits, pulses and some dark green leafy vegetables) are omitted. Finally, none of the available studies on the cost of nutritious diets analyzed the role of modern retailers such as supermarkets.

This dissertation addresses the highlighted research gaps by analyzing effects of modern food retailers on consumer diets and nutrition in urban Africa. The specific objectives of the dissertation are:

1. To examine the relationships between consumers' socioeconomic status, use of different modern and traditional retailers, and dietary patterns.

2. To analyze effects of using modern food retailers on adult and child diets and nutrition.

3. To analyze effects of using supermarkets on the affordability of recommended nutritious diets and dietary quality among demographic cohorts.

\subsection{Data and study context}

The data used in this dissertation were collected through a household survey conducted by the author in Lusaka, the capital city of Zambia, between April and July 2018. Like many other countries in sub-Saharan Africa, Zambia is an ideal setting for this study for several reasons. First, it is one of the southern African countries with rapid growth in modern retailers (Tschirley et al., 2015; Ziba and Phiri, 2017). For instance, our own review of internet sources supplemented by key local informant interviews revealed that the number of large shopping malls in Lusaka City increased from one in 1995 to 25 in 2018 (Table 1.1). Moreover, a substantial share $(43 \%)$ of the food consumed by urban households in Lusaka is purchased 
from modern retailers such as supermarkets and fast-food restaurants (Khonje and Qaim, 2019).

\section{Table 1.1: List of main shopping malls with modern food retailers in Lusaka City}

\begin{tabular}{|c|c|c|c|}
\hline No. & $\begin{array}{l}\text { Name of shopping } \\
\text { mall }\end{array}$ & $\begin{array}{l}\text { Location and surrounding compounds } \\
\text { or sections }\end{array}$ & $\begin{array}{l}\text { Modern food retailers: Hypermarkets, Supermarkets } \\
\text { (Fast-food restaurants in parentheses) }\end{array}$ \\
\hline 1 & Arcades & Roma, University of Zambia (UNZA) & Spurs \\
\hline 2 & Cairo & Central Lusaka & Shoprite (Food Fayre, Hungry Lion, Machachos) \\
\hline 3 & Chawama & Chawama, John Haward, Kuku & Spur \\
\hline 4 & Chazanga Shoprite & Chazanga, SOS & Shoprite \\
\hline 5 & Chilenje Shoprite & Chalala, Chilenje, Woodlands & $\begin{array}{l}\text { Choppies, Shoprite (Debonairs Pizza, MM Chickens, } \\
\text { Naaz) }\end{array}$ \\
\hline 6 & Choppies Complex & Kabulonga, Sundel, Zamtel Flats & Choppies \\
\hline 7 & Cosmopolitan & $\begin{array}{l}\text { Chawama, John Howard, Jon-Lengi, } \\
\text { Makeni, Misisi }\end{array}$ & $\begin{array}{l}\text { Game Stores, Shoprite (Chicken Inn, Galito's, } \\
\text { Hungry Lion, Mochachos, Pizza Hut) }\end{array}$ \\
\hline 8 & Cross Roads & $\begin{array}{l}\text { Cross Road, Kabulonga, Nyumba } \\
\text { Yanga, Sundel }\end{array}$ & Spurs (Gigibonta, Major Meat) \\
\hline 9 & Down Town & $\begin{array}{l}\text { Chibolya, Jon-Lengi, Kabwata, } \\
\text { Kamwala, Misisi }\end{array}$ & $\begin{array}{l}\text { Spurs (Big Bite, Debonairs Pizza, Down Town } \\
\text { Foods) }\end{array}$ \\
\hline 10 & East Park & $\begin{array}{l}\text { Childley, Kalingalinga, Kalundu, } \\
\text { Ng'ombe, Roma, UNZA }\end{array}$ & $\begin{array}{l}\text { Food Lover's, PicknPay (Fishaways, Gigibonta, } \\
\text { GoatnChips, Hungry Lion, KEG, Pizza Hut) }\end{array}$ \\
\hline 11 & Embassy & Chawama, Jon-Lengi, Makeni, Misisi & Embassy, Spurs (Papas, Piatto, Zorbas) \\
\hline 12 & Garden City & Avondole, Chelston & Food Lover's, PicknPay (Bushman, Foodano) \\
\hline 13 & $\begin{array}{l}\text { Kabulonga and } \\
\text { Melissa }\end{array}$ & Kabulonga & $\begin{array}{l}\text { Melissa, PicknPay (Debonairs Pizza, KFC, Nando's, } \\
\text { Subway) }\end{array}$ \\
\hline 14 & Levy Junction & $\begin{array}{l}\text { Central Lusaka, Chilulu, Evelyn Home } \\
\text { College, Gardens, Nippa, North Mead, } \\
\text { Roads Park, Thorn Park }\end{array}$ & $\begin{array}{l}\text { Food Lover's, PicknPay (Chicken Inn, Hungry Lion, } \\
\text { KFC, Pizza Inn, Wimpy) }\end{array}$ \\
\hline 15 & Makeni & Chawama, Jon-Lengi, Makeni, Misisi & $\begin{array}{l}\text { Food Lover's, PicknPay (Debonairs Pizza, KFC, } \\
\text { Nando's) }\end{array}$ \\
\hline 16 & Mama Betty Foxydale & Ngo'mbe, Roma & Spur (Debonairs Pizza, Gigibonta) \\
\hline 17 & Manda Hill & $\begin{array}{l}\text { Central Lusaka, Chilulu, Gardens, } \\
\text { Longacres, Olympia, Roads Park }\end{array}$ & $\begin{array}{l}\text { Shoprite, Game Stores (Bread Café, Debonairs Pizza, } \\
\text { Galito's, Hungry Lion, Mugg and Bean, My Asia, } \\
\text { Nando's, Pizza Inn, Steers, Subway, Vasila) }\end{array}$ \\
\hline 18 & Matero & Matero & Shoprite (Hungry Lion) \\
\hline 19 & Novara Great North & Chazanga, SOS & PicknPay (GoatnChips, Hungry Lion) \\
\hline 20 & PHI & Kaunda Square, PHI, Mtendere & PicknPay (Debonairs Pizza, King-Pie) \\
\hline 21 & SOS Spurs & Chazanga, SOS & Spur \\
\hline 22 & Twin Palm & Avondole, Chelston, Ibex, Salama Park & $\begin{array}{l}\text { Shoprite (Chicken Inn, Debonairs Pizza, Hungry } \\
\text { Lion) }\end{array}$ \\
\hline 23 & Waterfalls & Avondole, Chelston & Shoprite (Gigibonta, Hungry Lion) \\
\hline 24 & Woodlands & Chilenje, Kabulonga, Woodlands & $\begin{array}{l}\text { PicknPay (Creamy, Debonairs Pizza, Galito’s, } \\
\text { Nachies, O. Hagans, Pizza Inn) }\end{array}$ \\
\hline 25 & Zappa & Chawama & (Debonairs Pizza) \\
\hline
\end{tabular}

Notes: The main shopping malls that were operating in 2018 are included. Very small shopping malls are not included. Likewise, malls that were still under construction in 2018 are not included. The list was compiled by the authors based on internet search, personal visits, and key informant interviews.

Second, Zambia is characterized by a high prevalence of micronutrient malnutrition (Harris et al., 2019; Kaliwile et al., 2019). For instance, among women and children; 98\%, 34-55\%, $26 \%$, and $19 \%$ are deficient in vitamin $\mathrm{B}_{12}$, zinc, vitamin $\mathrm{A}$, and iron, respectively (Harris et 
al., 2019). Third, the share of adults who are overweight/obese has increased from $12 \%$ in 2002 to 32\% in 2017 (Steyn and Mchiza, 2014; Harris et al., 2019). Finally, stunting rates in Zambia are high, ranging from $40 \%$ to $50 \%$ (Harris et al., 2019). Overall, different forms of malnutrition coexist with rising overweight and obesity in Zambia.

We surveyed a total of 475 households from several compounds/sections in Lusaka City using a two-stage random sampling procedure. At the first stage, we purposively selected 14 compounds/sections as primary sampling units based on population distributions, the locations of major shopping malls (Table 1.1), as well as information from the Lusaka City council on mean income levels in the different compounds/sections of the city. To ensure that the sample is fairly representative of households in the urban parts of Lusaka, we selected compounds/sections from high (Avondole, Chalala, Kabulonga, Woodlands), middle (Chelston, Chilenje, Kabwata, PHI), and low (Chawama, Chazanga, Gardens, Kalingalinga, Kaunda Square, Ng'ombe) mean income levels. A spatial distribution of the surveyed compounds/sections and households is shown in Figure 1.1.

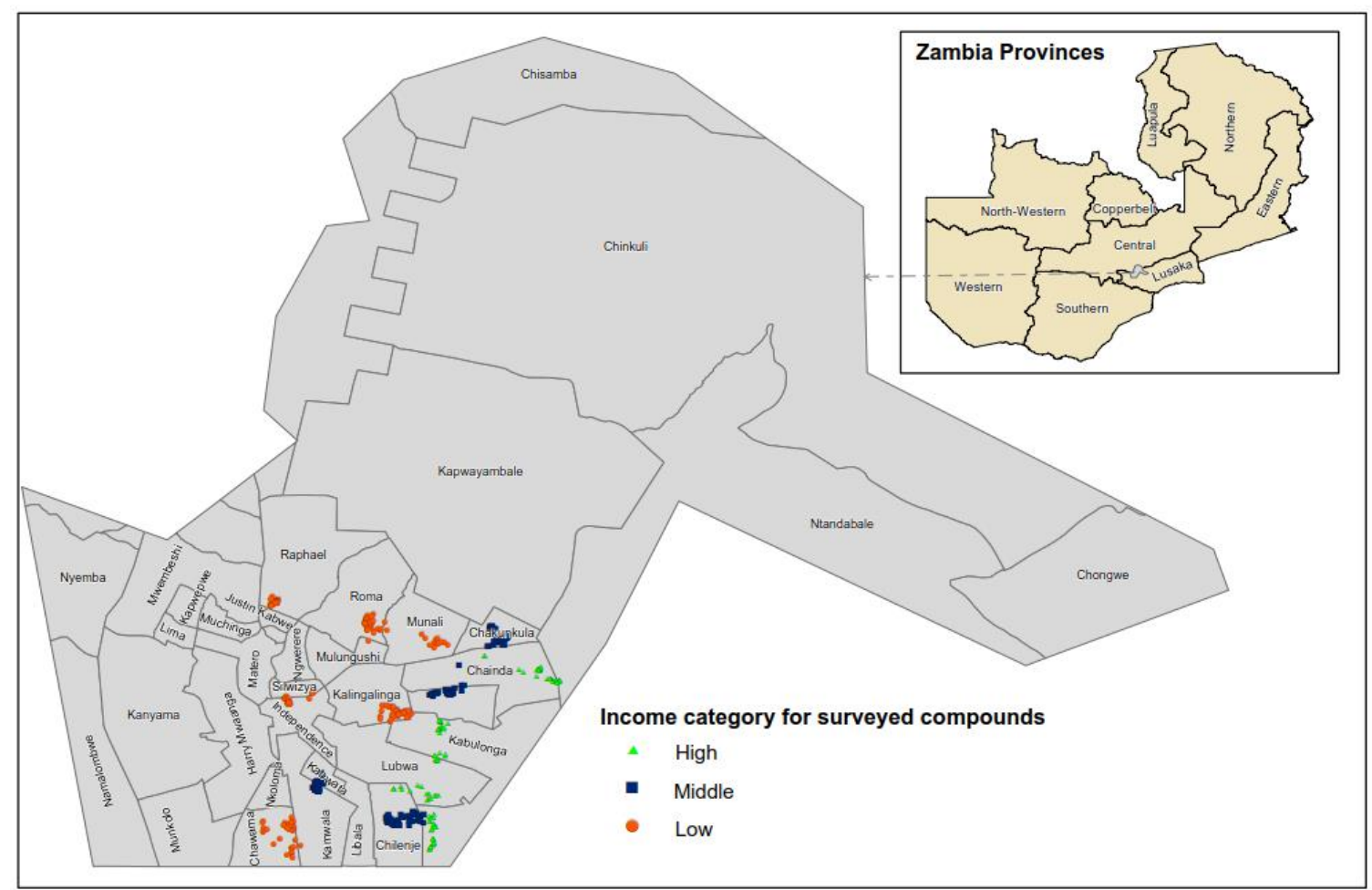

Figure 1.1: Map of Lusaka City with sampled compounds/sections and households 
At the second stage, depending on the size of the compound/section, we randomly sampled about 35 households from each compound/section for study participation. In each of the sampled household, we interviewed the household head or another adult respondent responsible for most food purchase decisions and food preparation. We recruited local enumerators to conduct face-to-face interviews in local languages. The enumerators were trained and supervised by the researchers. Food-intake data were captured at the individual level for up to four randomly selected members of each household: two adults ( $\geq 18$ years) and two children/adolescents ( 6 months - 18 years). Individual-level dietary data were collected through 24-hour dietary recalls; for small children the recall data were provided by the caregiver. Weight and height of all participating individuals were also measured. All participating adults provided written consent for themselves and for their children. The study was reviewed and approved by the Ethics Committee of the University of Goettingen.

In addition to the individual-level anthropometric and food-intake/dietary data, data on food consumption at the household level were collected through a seven-day recall using a list of 140 different food items typically consumed in the local setting, and capturing quantities and sources of each item. We also captured food price data for different food items at the household level through a seven-day recall. To account for individual and household level characteristics, our structured questionnaire also covered other sections including household demographic structure, economic activities, income, and consumption expenditures (see General Appendix).

\subsection{Dissertation outline}

The rest of this dissertation is organized as follows: Chapter 2 presents the first paper, analyzing the relationships between consumers' socioeconomic status, use of different modern and traditional retailers, and dietary patterns largely using the household level food consumption data from 475 households. The second paper is presented in Chapter 3, which analyzes effects of modern retailers on adult and child diets and nutrition. The second paper uses individual-level food-intake and anthropometric data from 930 adults and 499 children. The third paper, which analyzes effects of supermarkets on the affordability of recommended nutritious diets and dietary quality, is presented in Chapter 4 . The third paper uses food price data and the individual-level food-intake/dietary data from 1,429 observations: 295 men, 594 women, 240 boys, and 300 girls. Chapter 5 draws general conclusions and policy implications, based on all three papers. Limitations of the study are also discussed. The questionnaire developed for the study is presented in the General Appendix. 


\title{
2 Modernization of African food retailing and (un)healthy food consumption ${ }^{1}$
}

\begin{abstract}
Food environments in Africa are changing rapidly, with modern retailers - such as supermarkets, hypermarkets, and fast-food restaurants - gaining in importance. Changing food environments can influence consumers' food choices and dietary patterns. Recent research has suggested that the growth of supermarkets leads to more consumption of processed foods, less healthy diets, and rising obesity. However, relatively little is known about what type of consumers actually use modern supermarkets and to what extent. Moreover, focusing only on supermarkets may be misleading, as most consumers obtain their food from various modern and traditional retailers. We add to the literature by examining relationships between consumers' socioeconomic status, use of different modern and traditional retailers, and dietary patterns. The analysis uses household survey data from urban Zambia. Results show that two-thirds of the households use modern and traditional retailers simultaneously, but that richer households are more likely than poorer ones to use supermarkets and hypermarkets. Use of modern retailers is positively associated with higher consumption of ultra-processed foods, after also controlling for income and other socioeconomic factors. However, the use of traditional stores and kiosks is also positively associated with the consumption of ultra-processed foods, suggesting that modern retailers are not the only drivers of dietary transitions.
\end{abstract}

Keywords: Retail modernization; supermarkets; food consumption; diets; nutrition transition; Zambia.

\footnotetext{
${ }^{1}$ This paper has been co-authored with Matin Qaim (M.Q.). The research idea was jointly conceptualized and designed by I, Makaiko Gonapanyanja Khonje (M.G.K.) and M.Q.. M.G.K. collected, analyzed, and interpreted the data, and wrote the first draft of the manuscript. M.Q. commented on the paper at various stages and approved the final version - writing: review and editing. The paper is published in Sustainability, 11(16), 4306. DOI: https://doi.org/10.3390/su11164306.
} 


\subsection{Introduction}

Food systems in developing countries have been evolving rapidly in the last few decades, with a growing role played by modern retailers such as supermarkets, hypermarkets, convenience stores, and fast-food restaurants (Gómez and Ricketts, 2013; Popkin 2014; Reardon and Timmer, 2014; Andersson et al., 2015). The modernization of food systems is largely driven by consumer preference changes resulting from urbanization, income growth, and globalization (Tschirley et al., 2015; Minten et al., 2017; Qaim, 2017; Lu and Reardon, 2018; Reardon et al., 2019). However, at the same time consumer preferences and demand may also be shaped by changing food environments (Popkin, 2017; Laska et al., 2018; Popkin and Reardon, 2018). For example, a shift from traditional markets to modern supermarkets and hypermarkets has effects on the types of food offered, as well as on food variety, food prices, and shopping atmosphere, all of which may influence consumer choices (Asfaw, 2008; Hawkes, 2008; Reardon and Timmer, 2014; Odunitan-Wayas et al., 2018). Understanding the links between changing food environments and food consumption patterns is important to promote food security and healthy diets. This is especially true in Africa, where poverty and undernutrition are still widespread, but where being overweight and obesity are also on the rise (Ruel et al., 2017; Harris et al., 2019; Kroll et al., 2019).

Available research suggests that the modernization of food retailing may make calories more affordable for urban consumers but - at the same time - may foster the nutrition transition towards more highly processed foods that are rich in fat, sugar, and salt, but contain low amounts of micronutrients and other ingredients for healthy nutrition (Asfaw, 2011; Gómez and Ricketts, 2013; Popkin and Reardon, 2018; Freire and Rudkin, 2019). Recent studies with data from different countries in Africa, Asia, and Latin America suggest that the growth of supermarkets may contribute to increased consumption of processed foods and a higher body mass index (BMI), after also controlling for household income (Asfaw, 2008; Kimenju et al., 2015; Rischke et al., 2015; Umberger et al., 2015; Demmler et al., 2018; Kroll et al., 2019). However, especially in Africa, relatively little is known about what type of consumers actually use modern supermarkets and to what extent. Moreover, focusing only on supermarkets may be misleading, as most consumers obtain their food from various modern and traditional retailers (Berger and van Helvoirt, 2018; Lu and Reardon, 2018; Zhong et al., 2018; Vetter et al., 2019). 
Here, we add to the existing literature by analyzing more explicitly the associations between household socioeconomic status, the use of different types of retailers, and dietary patterns in urban Africa. In particular, we use household survey data from urban Zambia to analyze what type of socioeconomic characteristics are associated with the choice of modern and traditional food retailers, and to what extent the use of different retailers is associated with the consumption of processed and unprocessed foods, and products belonging to different healthy and unhealthy food groups. To our knowledge, this is the first study that looks into these issues with detailed data from Africa.

Zambia is an interesting empirical setting for this analysis, because it has recently experienced rapid growth of supermarkets, hypermarkets, and other modern retailers (Tschirley et al., 2015). Moreover, Zambia is experiencing a triple burden of malnutrition, where undernutrition and micronutrient malnutrition coexist with rising overweight and obesity (Steyn and Mchiza, 2014; Harris et al., 2019). Hence, our results may help to project how diets evolve with further changes in retail environments and what type of policy responses might be useful. We expect that the insights from Zambia can be useful also for other countries in Africa, where the modernization of the food retail sector is still in its earlier stages.

The rest of this paper is organized as follows. Section 2 provides an overview of the most important types of modern and traditional food retailers in Zambia. Section 3 explains materials and methods, including a description of the household survey, the measurement of key variables, and the econometric models used. Section 4 presents and discusses the results, while Section 5 provides the conclusions.

\subsection{Modern and traditional food retailers in Zambia}

Food retail environments in many African countries have been changing rapidly during the last 20 years, with a considerable growth of modern retailers such as supermarkets and hypermarkets (Tschirley et al., 2015; Ziba and Phiri, 2017). Zambia is one of the countries in the Southern African region with particularly high growth rates of modern retailers (PlanetRetail, 2017; Ziba and Phiri, 2017). For instance, our own review of internet sources supplemented by key informant interviews in the local context revealed that the number of large shopping malls in Lusaka City increased from one in 1995 to 25 in 2018 (Table 1.1). These shopping malls with a big variety of shops are also the main locations of supermarkets, hypermarkets, and fast-food restaurants. 
Most of these modern retailers are almost homogenous in product offerings across countries in Africa. For instance, supermarket retail giant like Shoprite; Africa's largest food retailer is operating more than 2738 outlets in 15 African countries (Angola, Botswana, Democratic Republic of Congo, eSwatini, Ghana, Lesotho, Madagascar, Malawi, Mauritius, Mozambique, Namibia, Nigeria, South Africa, Uganda and Zambia) (Shoprite Holdings, 2017). Smaller supermarkets and convenience stores are also found in other locations. In the following, we characterize the main types of modern food retailers that we also use in the empirical analysis below. We characterize the most important types of traditional food retailers as well. An overview of the key characteristics of each type of retailer is shown in Table 2.1. The classification builds on criteria similar to those used in previous studies (Rischke at al., 2015; Berger and Helvoirt, 2018; Demmler et al., 2018), partly adjusted to the local context based on key informant interviews.

The largest modern retailers are hypermarkets with a floor space of more than $200 \mathrm{~m}^{2}$. The main hypermarket chains in Lusaka are Game Stores, Cheers, and Choppies. Supermarkets are similar to hypermarkets, but are smaller with $100-200 \mathrm{~m}^{2}$ of floor space. Major supermarket chains in Lusaka include Shoprite and PicknPay, among others. Both hypermarkets and supermarkets are self-service stores with a wide range of fresh and processed products, including chilled and frozen foods. Convenience stores also belong to the category of modern retailers. They are also self-service in nature but are smaller $\left(<100 \mathrm{~m}^{2}\right)$ and offer a more limited range of food products. Finally, we include fast-food restaurants such as Hungry Lion, Debonairs Pizza, and KFC - in the group of modern retailers (Table 2.1).

Traditional food retailers include grocery stores, traditional markets, roadside markets, and neighborhood kiosks (Table 2.1). None of the traditional retailers have self-service options, all providing over-the-counter services. Traditional retailers are mostly owner-operated and do not belong to larger chains. Customers can negotiate prices to some extent and can usually also buy foods on credit. The range of products and brands offered by traditional retailers is smaller than that offered by modern retailers. Packaging sizes are also smaller. Sometimes traditional retailers repackage foods such as sugar, flour, or cooking oil, into very small packets, which are particularly demanded by poor customers. Traditional retailers rarely sell frozen and chilled foods, mostly due to lack of refrigeration facilities. 
Table 2.1: Key characteristics of different food retailers in Lusaka City, Zambia

\begin{tabular}{|c|c|c|c|c|c|c|c|c|}
\hline \multirow[b]{2}{*}{ Characteristic } & \multicolumn{4}{|c|}{ Modern retailers } & \multicolumn{4}{|c|}{ Traditional retailers } \\
\hline & Hypermarket & Supermarket & $\begin{array}{l}\text { Convenience } \\
\text { store }\end{array}$ & $\begin{array}{l}\text { Fast-Food } \\
\text { restaurant }\end{array}$ & $\begin{array}{l}\text { Grocery } \\
\text { store }\end{array}$ & $\begin{array}{l}\text { Traditional } \\
\text { market }\end{array}$ & $\begin{array}{l}\text { Roadside } \\
\text { market }\end{array}$ & $\begin{array}{l}\text { Neighborhood } \\
\text { kiosk }\end{array}$ \\
\hline Typical location & $\begin{array}{l}\text { Big shopping } \\
\text { mall }\end{array}$ & Big shopping mall & $\begin{array}{l}\text { Small shopping } \\
\text { mall }\end{array}$ & $\begin{array}{l}\text { Big shopping } \\
\text { mall or gas } \\
\text { station }\end{array}$ & $\begin{array}{l}\text { Very small } \\
\text { shopping mall }\end{array}$ & $\begin{array}{l}\text { Traditional } \\
\text { marketplace }\end{array}$ & Informal stall & $\begin{array}{l}\text { Formal or } \\
\text { informal stall }\end{array}$ \\
\hline Floor space $\left(\mathrm{m}^{2}\right)$ & $>200$ & $100-200$ & $<100$ & $10-30$ & $10-70$ & $1-10$ & $1-5$ & $1-5$ \\
\hline Modern cash tills & $4-15$ & $4-10$ & $<4$ & $<4$ & None & None & None & None \\
\hline Service type & Self-service & Self-service & Self-service & Pressing order & $\begin{array}{l}\text { Over the } \\
\text { counter }\end{array}$ & $\begin{array}{l}\text { Over the } \\
\text { counter }\end{array}$ & Over the counter & Over the counter \\
\hline Credit facility & No & No & No & No & Possible & Possible & Possible & Possible \\
\hline Promotions via media & Often & Often & Often & Often & Very rare & No & No & No \\
\hline Price discounts & $\begin{array}{l}\text { Occasional (e.g., } \\
\text { month ends) }\end{array}$ & $\begin{array}{l}\text { Occasional (e.g., } \\
\text { month ends) }\end{array}$ & $\begin{array}{l}\text { Occasional (e.g., } \\
\text { month ends) }\end{array}$ & $\begin{array}{l}\text { Occasional (e.g., } \\
\text { month ends) }\end{array}$ & Very rare & No & No & No \\
\hline Price negotiation & No & No & No & No & No & Often & Often & Often \\
\hline \multirow[t]{3}{*}{ Product range } & $\begin{array}{l}\text { Large variety of } \\
\text { food and non- } \\
\text { food products }\end{array}$ & $\begin{array}{l}\text { Large variety of } \\
\text { food and non-food } \\
\text { products }\end{array}$ & $\begin{array}{l}\text { Limited variety } \\
\text { of food and non- } \\
\text { food products }\end{array}$ & $\begin{array}{l}\text { Only fast food } \\
\text { products and } \\
\text { beverages }\end{array}$ & $\begin{array}{l}\text { Limited } \\
\text { variety of } \\
\text { food products }\end{array}$ & $\begin{array}{l}\text { Fairly large } \\
\text { variety of } \\
\text { legumes, } \\
\text { cereals, } \\
\text { vegetables }\end{array}$ & $\begin{array}{l}\text { Fairly large } \\
\text { variety of fruits } \\
\text { and vegetables }\end{array}$ & $\begin{array}{l}\text { Fairly large } \\
\text { variety of } \\
\text { legumes, cereals, } \\
\text { vegetables }\end{array}$ \\
\hline & $\begin{array}{l}\text { Large variety of } \\
\text { fruits and } \\
\text { vegetables }\end{array}$ & $\begin{array}{l}\text { Large variety of } \\
\text { fruits and } \\
\text { vegetables }\end{array}$ & $\begin{array}{l}\text { Limited variety } \\
\text { of fruits and } \\
\text { vegetables }\end{array}$ & $\begin{array}{l}\text { Limited variety } \\
\text { of vegetables }\end{array}$ & & & & \\
\hline & $\begin{array}{l}\text { Frozen, canned, } \\
\text { and cooked food }\end{array}$ & $\begin{array}{l}\text { Frozen, canned, } \\
\text { and cooked food }\end{array}$ & $\begin{array}{l}\text { Limited variety } \\
\text { of frozen and } \\
\text { canned food }\end{array}$ & & & & $\begin{array}{l}\text { Sometimes } \\
\text { cooked food }\end{array}$ & \\
\hline Packaging size & $\begin{array}{l}\text { Small to very } \\
\text { large }\end{array}$ & Small to very large & $\begin{array}{l}\text { Small to very } \\
\text { large }\end{array}$ & $\begin{array}{l}\text { Small to very } \\
\text { large }\end{array}$ & Small to large & $\begin{array}{l}\text { Very small to } \\
\text { small }\end{array}$ & $\begin{array}{l}\text { Very small to } \\
\text { small }\end{array}$ & $\begin{array}{l}\text { Very small to } \\
\text { small }\end{array}$ \\
\hline Repacking & No & No & No & No & No & Often & Often & Often \\
\hline $\begin{array}{l}\text { Key actors } \\
\text { (examples) }\end{array}$ & $\begin{array}{l}\text { Game Stores, } \\
\text { Cheers, Choppies }\end{array}$ & $\begin{array}{l}\text { Shoprite, } \\
\text { PicknPay, Food } \\
\text { Lover's, Spurs }\end{array}$ & Numerous & $\begin{array}{l}\text { Hungry Lion, } \\
\text { Debonairs Pizza, } \\
\text { KFC, KEG } \\
\end{array}$ & Numerous & $\begin{array}{l}\text { Soweto, } \\
\text { Compound } \\
\text { Markets } \\
\end{array}$ & Numerous & Numerous \\
\hline
\end{tabular}




\subsection{Materials and methods}

\subsubsection{Household survey}

The data used in this study were collected through a household survey in Lusaka, the capital city of Zambia, between April and July 2018. We surveyed a total of 475 households using a two-stage random sampling procedure. At the first stage, we purposively selected 14 compounds or sections within Lusaka urban. These compounds were selected based on the locations of major shopping malls as well as information provided by the City Council on mean income levels in the different compounds or sections. Based on population distributions, we selected four compounds with high mean income levels (Avondole, Chalala, Kabulonga, Woodlands), four compounds with medium income levels (Chelston, Chilenje, Kabwata, PHI), and six compounds with low income levels (Chawama, Chazanga, Gardens, Kalingalinga, Kaunda Square, Ng'ombe). At the second stage, depending on compound size, we randomly sampled around 35 households from each compound for study participation. The spatial distribution of selected compounds and households is shown in Figure 1.1. The sample should be fairly representative of households in the urban parts of Lusaka.

In each of the sample households, we carried out a face-to-face interview with the household head or another adult responsible for food purchase decisions. The computer-aided structured interviews were conducted in the local language by a small team of interviewers that we recruited, trained, and supervised. The questionnaire that we had developed for this purpose captured general economic and socio-demographic information of the household and its members. Food consumption data were collected through a 7-day household-level recall, using a detailed list of food items typically consumed in the local setting. In addition to food quantities and expenditures, we also collected data on the processing level and the source of each food item, focusing particularly on the different modern and traditional retailers. These data were used to construct various key variables, as discussed below.

\subsubsection{Measurement of key variables}

We are interested in analyzing the relationship between socioeconomic characteristics and use of different retailers. Socioeconomic characteristics of interest include household income levels, education, gender, and age of the household head, household size and structure, ethnicity, religion, car ownership, among others. Previous research showed that these 
characteristics can influence the decision of which retailers to use (e.g., Asfaw, 2008; Rischke et al., 2015; Umberger et al., 2015; Demmler et al., 2018; Odunitan-Wayas et al., 2018; Rupa et al., 2019). The use of different retailers is measured through a set of dummy variables capturing whether or not the household purchased any food from a particular type of retailer during the 7-day recall period. In addition to the retailer dummies, we also examine the share of the total household food budget spent in different retail outlets.

We are also interested in analyzing associations between the use of different retailers and dietary patterns. One way of looking at dietary patterns is through classifying all food items consumed by their level of processing. We differentiate between unprocessed foods, primary processed foods, and ultra-processed foods (Demmler et al., 2018). For these three processing levels, we calculate household expenditures and food expenditure shares. Unprocessed foods include wholegrain cereals and pulses, fresh fruits and vegetables, eggs and fresh milk, among others. Primary processed foods include milled cereals and fresh meat and fish. Ultraprocessed foods include bread, pasta, dairy products, sausages and meat products, soft drinks, sweets, and other ready-made dishes and snacks (Table A2.1 in the Supplementary material). Ultra-processed foods are generally considered less healthy than unprocessed foods, because they often have high sugar, fat, and salt contents, and low fiber and micronutrient contents. Research has shown that high consumption of ultra-processed foods is associated with obesity and increased risks of chronic diseases such as coronary heart diseases, stroke, and diabetes (Monteiro et al., 2010; Beatty et al., 2014; Steyn and Mchiza, 2014; Harris et al., 2019).

Separate indicators of dietary patterns that we use are the quantities of different food groups consumed by the households during the 7-day recall period. We use the following food groups: cereals and tubers, legumes, fruits, vegetables, meat and fish, dairy products, eggs, oils and fats, and sugar and sugar-sweetened beverages. While the last two food groups are considered as rather unhealthy, the others contain important nutrients and can therefore contribute to healthy nutrition.

\subsubsection{Statistical analysis}

We start the analysis by calculating descriptive statistics for the use of modern retailers and dietary patterns and comparing between households of different socioeconomic status. For this purpose, we subdivide the sample into three groups of almost equal size, namely the 
lower, middle, and upper income terciles. In addition, we use regression models to analyze the associations of interest more formally.

To analyze the socioeconomic factors that influence the use of different types of retailers, we estimate models of the following type:

$$
\boldsymbol{F} \boldsymbol{R}_{i}=\alpha+\beta^{\prime} \boldsymbol{X}_{i}+\varepsilon_{i}
$$

where $\boldsymbol{F} \boldsymbol{R}_{i}$ is a vector of the types of food retailers that household $i$ used during the 7-day recall period, $\boldsymbol{X}_{i}$ is a vector of socioeconomic variables, and $\varepsilon_{i}$ is a random error term. $\boldsymbol{F} \boldsymbol{R}_{i}$ is measured through a set of dummy variables, one for each of the modern and traditional retailers considered, so that we use a probit specification to estimate Equation (2.1). Households can use more than one type of retailer, and the decisions for different retailers are likely correlated. We use a multivariate probit model to account for possible error correlation between the equations for different retailers (Cappellari and Jenkins, 2003).

Next, we analyze how far the use of particular retailers is associated with more or less healthy dietary patterns by estimating regression models of the following type:

$$
\boldsymbol{D} \boldsymbol{P}_{i}=\gamma+\delta^{\prime} \boldsymbol{F} \boldsymbol{R} \boldsymbol{S}_{i}+\rho^{\prime} \boldsymbol{X}_{i}+u_{i}
$$

where $\boldsymbol{D} \boldsymbol{P}_{i}$ characterizes the observed dietary pattern of household $i$, and $u_{i}$ is the random error term. $\boldsymbol{F} \boldsymbol{R} \boldsymbol{S}_{i}$ is a vector of variables representing the food expenditure shares of each of the retailers, and $\boldsymbol{X}_{i}$ is a vector of socioeconomic characteristics. In one set of regressions, $\boldsymbol{D} \boldsymbol{P}_{i}$ will characterize expenditures for foods with different processing levels, while in another set of regressions $\boldsymbol{D} \boldsymbol{P}_{i}$ will characterize the consumption of different healthy and unhealthy food groups.

For the processing level equations, we use an ordinary least squares (OLS) estimator. As error term correlation between the different equations is possible, we also use a seemingly unrelated regression (SUR) estimator to compare the results. Furthermore, in addition to estimates with the full sample, we estimate separate models for households below and above the poverty line, as the role of modern retailers may potentially differ by socioeconomic status. For the food group equations, we use a Tobit estimator, because the consumption quantities are left-censored at zero. To account for the heterogeneity among the sampled households, for all models, standard errors are clustered at the level of city compounds. 
We start estimating the models in Equation (2.2) by only considering one food retailer in $\boldsymbol{F} \boldsymbol{R} \boldsymbol{S}_{i}$, namely supermarkets. This is similar to previous studies that have analyzed the effects of supermarkets on diets and nutrition (Asfaw, 2008; Rischke et al., 2015; Umberger et al., 2015; Demmler et al., 2018; Rupa et al., 2019). However, conclusions based on such models that only consider the use of supermarkets may be incomplete, as households typically use various types of retailers. To demonstrate this, we re-estimate the same models with all types of retailers included. We note that the use of food retailers (vector $\boldsymbol{F} \boldsymbol{R} \boldsymbol{S}_{i}$ ) is endogenous, so the estimated $\delta$ coefficients from Equation (2.2) should not be interpreted as causal effects. Using instruments to deal with possible endogeneity bias would be possible in principle, but is difficult in our case, with a total of eight endogenous variables. We were unable to identify eight valid instruments, which is why we interpret the estimated coefficients only in terms of associations.

\subsection{Results and discussion}

\subsubsection{Household socioeconomic characteristics}

Table 2.2 shows summary statistics for selected household socioeconomic variables (additional variables are shown in Table A2.2 in the Supplementary material). Average annual per capita incomes range from US\$ 410 in the lowest tercile to more than US\$5,000 in the highest tercile. Twenty-seven percent of the sample households fall below the international poverty line of US\$1.90 per capita in purchasing power parity terms (World Bank, 2019). We observe large differences between the income terciles in terms of education, occupation, and car ownership. While only $3 \%$ of the households in the lowest tercile own a car, in the highest tercile the share is $60 \%$.

The middle and lower parts of Table 2.2 show food consumption patterns. The average consumption of cereals, tubers, and legumes does not differ much between the three income terciles, whereas the consumption of most of the other food groups increases considerably with income, as one would expect. Noteworthy is the very low consumption of fruits in all three income terciles. Many of the households consume fruits only occasionally. In terms of processing levels, for the sample as a whole, $25 \%$ of the food expenditures are for unprocessed foods, $40 \%$ for primary processed foods, and $35 \%$ for ultra-processed foods. 
Table 2.2: Socioeconomic characteristics and food consumption patterns

\begin{tabular}{|c|c|c|c|c|}
\hline & \multirow{2}{*}{ Full sample } & \multicolumn{3}{|c|}{ By income tercile } \\
\hline & & Lowest & Middle & Highest \\
\hline \multicolumn{5}{|l|}{ Socioeconomic characteristics } \\
\hline \multirow[t]{2}{*}{ Household income (US\$/year) } & 10691.40 & 1855.67 & 7548.14 & 22920.93 \\
\hline & $(12163.16)$ & $(1036.68)$ & $(2134.58)$ & $(14347.06)$ \\
\hline \multirow[t]{2}{*}{ Household size (members) } & 4.52 & 4.53 & 4.47 & 4.56 \\
\hline & $(1.63)$ & $(1.79)$ & $(1.66)$ & $(1.43)$ \\
\hline \multirow[t]{2}{*}{ Male household head (dummy) } & 0.53 & 0.46 & 0.49 & 0.65 \\
\hline & $(0.50)$ & $(0.50)$ & $(0.50)$ & $(0.48)$ \\
\hline \multirow[t]{2}{*}{ Education of household head (dummy) } & 12.03 & 9.48 & 11.88 & 14.77 \\
\hline & $(3.93)$ & $(3.62)$ & $(3.46)$ & $(2.71)$ \\
\hline \multirow[t]{2}{*}{ Office job (dummy, any household member) } & 0.45 & 0.10 & 0.51 & 0.74 \\
\hline & $(0.50)$ & $(0.30)$ & $(0.50)$ & $(0.44)$ \\
\hline \multirow[t]{2}{*}{ Car ownership (dummy) } & 0.28 & 0.03 & 0.21 & 0.60 \\
\hline & $(0.45)$ & $(0.16)$ & $(0.41)$ & $(0.49)$ \\
\hline \multicolumn{5}{|l|}{ Food consumption } \\
\hline \multirow[t]{2}{*}{ Cereals and tubers (kg/week) } & 11.88 & 11.23 & 11.45 & 12.97 \\
\hline & $(5.20)$ & $(5.48)$ & $(4.56)$ & $(5.38)$ \\
\hline \multirow[t]{2}{*}{ Legumes (kg/week) } & 1.22 & 1.27 & 1.34 & 1.03 \\
\hline & (1.59) & $(1.55)$ & $(1.83)$ & $(1.34)$ \\
\hline \multirow[t]{2}{*}{ Fruits (kg/week) } & 0.28 & 0.22 & 0.26 & 0.37 \\
\hline & $(0.82)$ & $(0.73)$ & $(0.83)$ & $(0.89)$ \\
\hline \multirow[t]{2}{*}{ Vegetables (kg/week) } & 4.22 & 4.36 & 4.57 & 3.70 \\
\hline & $(3.74)$ & $(3.78)$ & $(3.87)$ & $(3.52)$ \\
\hline \multirow[t]{2}{*}{ Meat and fish (kg/week) } & 4.81 & 3.38 & 4.85 & 6.24 \\
\hline & $(3.45)$ & $(2.86)$ & $(3.24)$ & $(3.64)$ \\
\hline \multirow[t]{2}{*}{ Dairy products (kg/week) } & 0.61 & 0.25 & 0.48 & 1.11 \\
\hline & $(1.27)$ & $(0.65)$ & $(1.01)$ & $(1.74)$ \\
\hline \multirow[t]{2}{*}{ Eggs (kg/week) } & 0.44 & 0.28 & 0.34 & 0.69 \\
\hline & $(0.77)$ & $(0.64)$ & $(0.67)$ & $(0.92)$ \\
\hline \multirow[t]{2}{*}{ Oils and fats (kg/week) } & 0.69 & 0.65 & 0.72 & 0.70 \\
\hline & $(0.60)$ & $(0.58)$ & $(0.60)$ & $(0.62)$ \\
\hline \multirow[t]{2}{*}{ Sugar, sweetened beverages (kg/week) } & 1.68 & 1.28 & 1.65 & 2.13 \\
\hline & $(2.59)$ & (1.99) & $(2.31)$ & $(3.26)$ \\
\hline \multicolumn{5}{|l|}{ Food expenditures } \\
\hline \multirow[t]{2}{*}{ Total weekly food expenditure (ZMW/capita) } & 112.46 & 96.32 & 115.61 & 125.69 \\
\hline & $(62.98)$ & $(65.99)$ & $(59.37)$ & $(60.18)$ \\
\hline \multirow[t]{2}{*}{ Unprocessed foods (\%) } & 0.25 & 0.29 & 0.25 & 0.20 \\
\hline & $(0.14)$ & $(0.16)$ & $(0.13)$ & $(0.12)$ \\
\hline \multirow[t]{2}{*}{ Primary processed foods $(\%)$} & 0.40 & 0.35 & 0.40 & 0.45 \\
\hline & $(0.17)$ & $(0.18)$ & $(0.17)$ & $(0.15)$ \\
\hline \multirow[t]{2}{*}{ Ultra-processed foods (\%) } & 0.35 & 0.36 & 0.35 & 0.35 \\
\hline & $(0.14)$ & $(0.14)$ & $(0.14)$ & $(0.12)$ \\
\hline Observations & 475 & 159 & 160 & 156 \\
\hline
\end{tabular}

Notes: Mean values are shown with standard deviations in parentheses. ZMW, Zambia Kwacha (local currency). The average exchange rate was ZMW $9.87=$ US\$ 1 in mid-2018. Descriptive statistics of additional variables are shown in Table A2.2 in the Supplementary material.

Strikingly, the expenditure share for ultra-processed foods does not increase with income, emphasizing that the purchase and consumption of these types of foods are very common for all types of households in Lusaka City. 


\subsubsection{Role of modern and traditional retailers}

Table 2.3 shows the proportion of households using the different modern and traditional retailers. This refers to the sources of the foods consumed during the 7-day recall period used in the household survey. While the regular use of hypermarkets and fast-food restaurants is relatively low, the majority of all households (73\%) used supermarkets. Even more (75\%) used at least one of the modern food retailers. As expected, the use of modern retailers increases considerably from the lowest to the highest tercile. In the highest tercile, almost all

Table 2.3: Proportion of households using different modern and traditional retailers

\begin{tabular}{|c|c|c|c|c|}
\hline & \multirow{2}{*}{ Full sample } & \multicolumn{3}{|c|}{ By income tercile } \\
\hline & & Lowest & Middle & Highest \\
\hline \multicolumn{5}{|l|}{ Modern retailers } \\
\hline Hypermarkets & 0.05 & 0.01 & 0.04 & 0.12 \\
\hline Supermarkets & 0.73 & 0.48 & 0.78 & 0.92 \\
\hline Convenience store & 0.12 & 0.12 & 0.09 & 0.16 \\
\hline Fast-food restaurant & 0.02 & 0.01 & 0.01 & 0.04 \\
\hline \multicolumn{5}{|l|}{ Traditional retailers } \\
\hline Grocery stores & 0.45 & 0.64 & 0.43 & 0.28 \\
\hline Traditional market & 0.73 & 0.70 & 0.74 & 0.74 \\
\hline Roadside market & 0.36 & 0.54 & 0.33 & 0.20 \\
\hline Neighborhood kiosk & 0.20 & 0.17 & 0.20 & 0.23 \\
\hline Observations & 475 & 159 & 160 & 156 \\
\hline
\end{tabular}

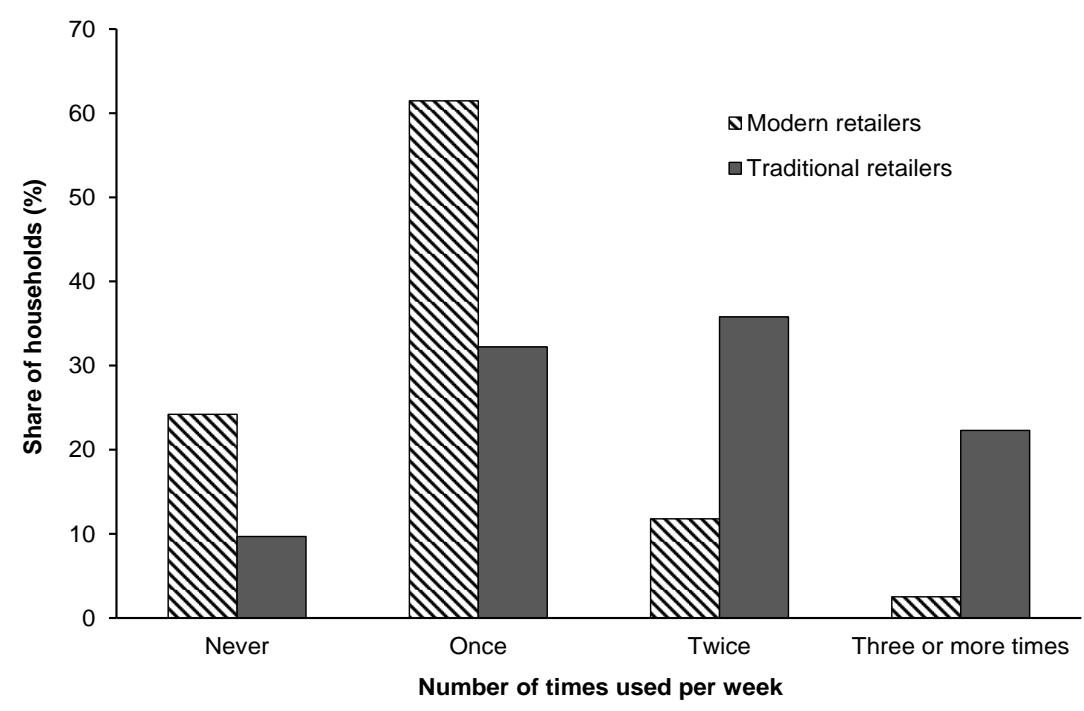

Figure 2.1: Frequency of use of modern and traditional retailers in Lusaka City 
households used at least one of the modern retailers. Most households in all income terciles used more than one type of retailer during the 7-day recall period. Two-thirds used both modern and traditional retailers.

Figure 2.1 shows that the average frequency of traditional retailer use is higher than that of modern retailer use. Many households make one larger purchase in a supermarket or hypermarket once a week and then purchase additional foods from traditional retailers whenever needed during the week.

The finding that many consumers use both modern and traditional retailers is consistent with a recent study for Nairobi (Berger and van Helvoirt, 2018) and also with theoretical predictions for a setting with large socioeconomic heterogeneity ( $\mathrm{Lu}$ and Reardon, 2018). The use of some traditional retailers decreases with rising household income, which is especially true for grocery stores and roadside markets. In contrast, the use of traditional markets and kiosks does not decrease with rising income (Table 2.3).

Figure 2.2 shows the distribution of household food expenditure shares by type of retailer. For the sample as a whole, $42 \%$ of the food expenditures are made for purchases from modern retailers. This is very high when compared to most other African countries, even when only looking at urban areas (Qaim, 2017). The rest of the household food budgets are spent (58\%) in traditional retail outlets. Notable differences are observed between the three income terciles. While households in the highest tercile make $63 \%$ of their food expenditures in modern retailers, for households in the lowest tercile this share is only around $20 \%$.

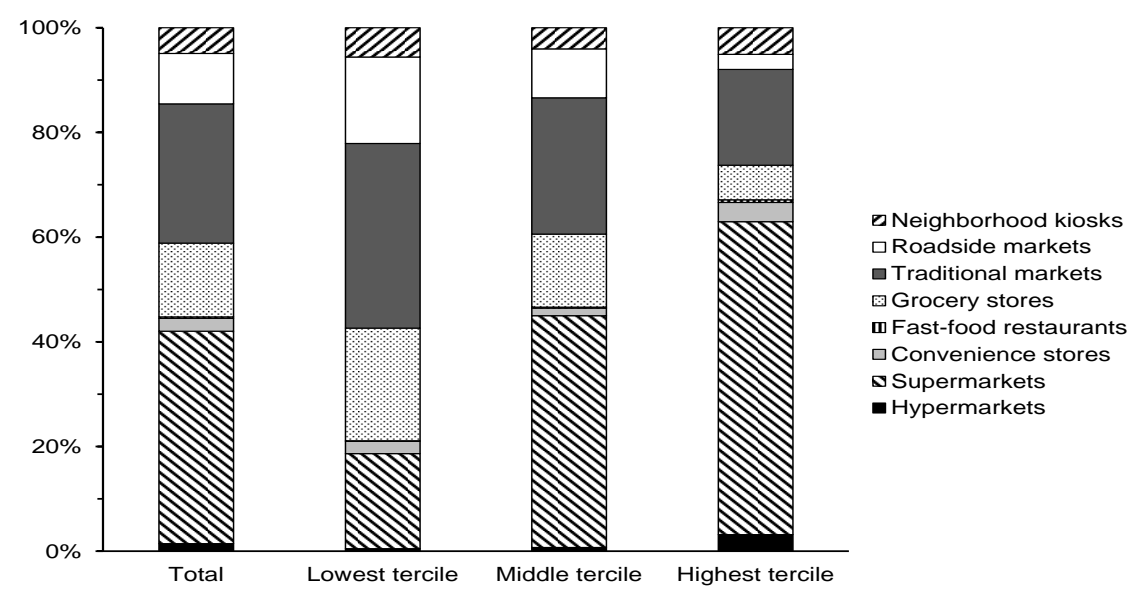

Figure 2.2: Household food expenditure shares spent in different retail outlets in Lusaka City 
This is in line with Figuié and Moustier (2009), and Berger and van Helvoirt (2018) who found that poor households use modern retailers less extensively than rich households in Vietnam, and Kenya, respectively. Among the modern retailers, supermarkets account for the lion's share of food expenditures for all households in Lusaka.

\subsubsection{Factors influencing the use of modern retailers}

We now look at the estimation results from the multivariate probit model to analyze factors influencing the household decision regarding whether or not to use particular types of retailers (see Equation (2.1) above). Average estimated marginal effects are shown in Table 2.4. Household income has a positive effect on the likelihood of using modern supermarkets and hypermarkets and a negative effect on the likelihood of traditional grocery stores and roadside markets, also after controlling for a number of other household characteristics. As mentioned earlier, occasionally, many households make one larger purchase in a modern retailer and often buy smaller food quantities from a traditional retailer (e.g., see Figure 2.1). In contrast, and consistent with the descriptive statistics above, the likelihood of using traditional markets and kiosks does not decrease with rising income. The use of traditional kiosks even increases when household income rises.

Education also affects the use of modern supermarkets positively. Similarly, more education tends to increase the use of fast-food restaurants. This latter result may be surprising, because fast food dishes are typically not very healthy, and better-educated households are generally expected to know more about healthy nutrition. On the other hand, education may also be a proxy of more exposure to global influences and lifestyles, which may contribute to a certain preference for westernized diets. Furthermore, better-educated consumers are often more conscious about food safety issues. In many developing countries, modern retailers and restaurants are perceived to fulfill higher food safety standards than their traditional counterparts (Mergenthaler et al., 2009; Gorton et al., 2011; Schipmann and Qaim, 2011; Wertheim-Heck et al., 2015). This could also explain why households with more education are significantly less likely to use traditional grocery stores, roadside markets, and kiosks. For instance, each additional year of schooling reduces the likelihood of purchasing food from a roadside market by 2.8 percentage points. 
Table 2.4: Factors influencing the use of different food retailers (Multivariate Probit Model)

\begin{tabular}{|c|c|c|c|c|c|c|c|c|}
\hline & \multicolumn{4}{|c|}{ Modern retailers } & \multicolumn{4}{|c|}{ Traditional retailers } \\
\hline & Hypermarket & Supermarket & $\begin{array}{c}\text { Convenience } \\
\text { store }\end{array}$ & $\begin{array}{l}\text { Fast-Food } \\
\text { restaurant }\end{array}$ & $\begin{array}{l}\text { Grocery } \\
\text { store }\end{array}$ & $\begin{array}{c}\text { Traditional } \\
\text { market }\end{array}$ & $\begin{array}{c}\text { Roadside } \\
\text { market }\end{array}$ & $\begin{array}{l}\text { Neighborhood } \\
\text { kiosk }\end{array}$ \\
\hline \multirow[t]{2}{*}{ Income (log) } & $0.031 * *$ & $0.063 * * *$ & 0.027 & 0.011 & $-0.045^{* *}$ & 0.015 & $-0.043 * *$ & $0.072 * * *$ \\
\hline & $(0.015)$ & $(0.017)$ & $(0.018)$ & $(0.009)$ & $(0.022)$ & $(0.022)$ & $(0.021)$ & $(0.022)$ \\
\hline \multirow[t]{2}{*}{ Household size } & -0.004 & $-0.031 * *$ & $0.019^{*}$ & $0.009 *$ & $0.054 * * *$ & 0.017 & $0.044 * * *$ & -0.001 \\
\hline & $(0.009)$ & $(0.012)$ & $(0.011)$ & $(0.005)$ & $(0.016)$ & $(0.016)$ & $(0.015)$ & $(0.026)$ \\
\hline \multirow[t]{2}{*}{ Education (years) } & -0.002 & $0.025 * * *$ & -0.007 & $0.006^{* *}$ & $-0.021 * * *$ & 0.000 & $-0.028 * * *$ & $-0.012 *$ \\
\hline & $(0.005)$ & $(0.006)$ & $(0.005)$ & $(0.003)$ & $(0.007)$ & $(0.007)$ & $(0.007)$ & $(0.006)$ \\
\hline \multirow[t]{2}{*}{ Age (years) } & 0.000 & 0.000 & 0.001 & -0.001 & -0.002 & -0.001 & -0.002 & 0.001 \\
\hline & $(0.001)$ & $(0.001)$ & $(0.001)$ & $(0.001)$ & $(0.002)$ & $(0.002)$ & $(0.002)$ & $(0.002)$ \\
\hline \multirow[t]{2}{*}{ Male (dummy) } & 0.007 & $-0.088 * *$ & 0.022 & -0.008 & $0.105^{* *}$ & 0.009 & $0.168 * * *$ & $0.091 * *$ \\
\hline & $(0.023)$ & $(0.035)$ & $(0.032)$ & $(0.015)$ & $(0.044)$ & $(0.041)$ & $(0.043)$ & $(0.038)$ \\
\hline \multirow{2}{*}{ Office job (dummy) } & 0.004 & $0.109 * *$ & -0.015 & $-0.033 *$ & -0.072 & $0.123^{* *}$ & $-0.125 * *$ & $-0.100 * *$ \\
\hline & $(0.027)$ & $(0.043)$ & $(0.038)$ & $(0.018)$ & $(0.052)$ & $(0.053)$ & $(0.051)$ & $(0.046)$ \\
\hline \multirow[t]{2}{*}{ Car ownership (dummy) } & $0.054 * *$ & $0.157 * * *$ & $0.086 * *$ & 0.010 & $-0.124 * *$ & $-0.113 * *$ & 0.008 & -0.012 \\
\hline & $(0.024)$ & $(0.058)$ & $(0.038)$ & $(0.017)$ & $(0.056)$ & $(0.054)$ & $(0.054)$ & $(0.048)$ \\
\hline \multirow[t]{2}{*}{ Adolescents (dummy) } & 0.014 & 0.053 & -0.003 & 0.010 & -0.017 & 0.050 & -0.060 & 0.043 \\
\hline & $(0.022)$ & $(0.037)$ & $(0.034)$ & $(0.015)$ & $(0.046)$ & $(0.045)$ & $(0.045)$ & $(0.040)$ \\
\hline \multirow[t]{2}{*}{ Children (dummy) } & -0.002 & -0.019 & 0.015 & 0.011 & 0.030 & 0.061 & 0.009 & 0.016 \\
\hline & $(0.022)$ & $(0.040)$ & $(0.034)$ & $(0.016)$ & $(0.048)$ & $(0.046)$ & $(0.046)$ & $(0.042)$ \\
\hline \multirow[t]{2}{*}{ Chewa (dummy) } & -0.035 & -0.011 & -0.008 & -0.176 & $0.107 *$ & -0.024 & -0.098 & 0.007 \\
\hline & $(0.047)$ & $(0.050)$ & $(0.048)$ & $(6.286)$ & $(0.064)$ & $(0.063)$ & $(0.062)$ & $(0.055)$ \\
\hline \multirow[t]{2}{*}{ Tonga (dummy) } & $0.058 * *$ & $-0.118 * *$ & 0.067 & 0.008 & 0.005 & 0.005 & -0.057 & -0.008 \\
\hline & $(0.024)$ & $(0.048)$ & $(0.041)$ & $(0.017)$ & $(0.058)$ & $(0.060)$ & $(0.056)$ & $(0.050)$ \\
\hline \multirow[t]{2}{*}{ Catholic (dummy) } & $0.039 *$ & $-0.089 * *$ & 0.052 & 0.020 & 0.078 & 0.036 & -0.041 & 0.067 \\
\hline & $(0.023)$ & $(0.039)$ & $(0.033)$ & $(0.016)$ & $(0.049)$ & $(0.047)$ & $(0.047)$ & $(0.041)$ \\
\hline Seventh Day Adventist & -0.017 & 0.031 & -0.059 & 0.001 & 0.010 & 0.083 & -0.049 & -0.007 \\
\hline (dummy) & $(0.028)$ & $(0.053)$ & $(0.049)$ & $(0.014)$ & $(0.060)$ & $(0.063)$ & $(0.059)$ & $(0.058)$ \\
\hline
\end{tabular}

Notes: Average marginal effects are shown with standard errors in parenthesis. Number of observations $=475$. Log pseudo likelihood $=-1460$, and Wald $\chi^{2}(104)=364$. Bemba and Protestant are used as a reference group for ethnicity - Chewa and Tonga, and religion status - Catholic and Seventh Day Adventist, respectively. * significant at the $10 \%$ level; ** significant at the $5 \%$ level; *** significant at the $1 \%$ level. 
The other results in Table 2.4 show that household size has a negative effect on using supermarkets and a positive effect on using traditional grocery stores and roadside markets. These results are probably related to shop opening hours and convenience. Supermarkets and hypermarkets have longer and more reliable opening hours than most traditional retailers. Furthermore, given the wide variety of products offered in supermarkets and hypermarkets, one-stop shopping is easily possible, which is much less the case for traditional retailers. These conditions make supermarkets particularly convenient for people with time constraints. In larger households, time constraints may be less severe, at least for some household members, so that the use of traditional retailers is more easily possible. Time constraints could also explain why people with an office job are more likely to use supermarkets and less likely to use roadside markets and kiosks. Also in line with this, is the fact that male-headed households are less likely to use supermarkets and more likely to use traditional retailers than female-headed households. Female household heads are typically the main income earners of the family and the main homemakers simultaneously, which means that only a small amount of time is available for shopping.

Other socioeconomic characteristics that seem to influence the choice of modern and traditional retailers include car ownership, ethnicity, and religion (Table 2.4). Car ownership increases the likelihood of using modern retailers and decreases the likelihood of using traditional retailers. This is unsurprising, given that most of the supermarkets and hypermarkets are located in larger shopping malls that typically also provide easy access by car and parking space. The patterns for ethnicity and religion are probably related to geographic clustering. On average, Tonga and Catholic households live more closely to shopping malls with a large hypermarket.

The error term correlation matrix for the multivariate probit model is shown in Table A2.3 in the Supplementary material. The null hypothesis of zero correlation between the equations for the different retailers is rejected, suggesting that the multivariate probit specification is preferred over separate single equation probit models. The correlation coefficients shown in Table A2.3 can also be interpreted economically. A positive correlation means that consumers use both retailers in a complementary way. This is observed, for instance, between hypermarkets and modern convenience stores. While the former are used for making largequantity purchases, the latter are used for making complementary smaller purchases. A 
positive correlation is also observed between traditional grocery stores and neighborhood kiosks.

On the other hand, we also observe negative correlations, for instance between modern supermarkets and traditional grocery stores, indicating that these types of retailers are rather considered substitutes. Both offer a similar range of products, but the variety in modern supermarkets is larger. These results indicate that traditional grocery stores may suffer the most from a shrinking customer base with the continued expansion of modern supermarkets. Other traditional retailers - such as traditional markets and neighborhood kiosks - may also be affected negatively by further supermarket expansion, but to a lesser extent than grocery stores. These types of competitive relationships between modern and traditional retailers are in line with earlier observations in Asia, Europe, and the USA (Suryadarma et al., 2010; Schipmann and Qaim, 2011; Stewart and Dong, 2018; Zhong et al., 2018; Hovhannisyan et al., 2019).

\subsubsection{Associations between retailers and food processing levels}

We now estimate the associations between the use of different retailers and household dietary patterns (see Equation (2.2) above), starting with the disaggregation of the foods consumed by processing level. Results are summarized in Table 2.5 (full estimation results are shown in Table A2.4 in the Supplementary material).

The results in Table 2.5 are single-equation OLS estimates. We also used SUR as an alternative estimator to account for possible correlation between the error terms. SUR results are shown in Table A2.5 in the Supplementary material. They are very similar to the OLS estimates, only that the SUR estimator cannot easily be combined with the cluster correction of standard errors. The upper part of Table 2.5 (panel A) shows models where supermarkets are considered as the only retailer variable.

The higher the share of food expenditures made in supermarkets, the higher the consumption of ultra-processed and primary processed foods, and the lower the consumption of unprocessed foods (panel A, Table 2.5). These results are consistent with previous studies in Guatemala and Kenya showing that the use of supermarkets contributes to a shift from the consumption of unprocessed to highly processed foods (Asfaw, 2008; Kimenju et al., 2015; Rischke et al., 2015). As mentioned, the consumption of ultra-processed foods was shown to 
Table 2.5: Associations between the use of different retailers and food processing levels

\begin{tabular}{lccc}
\hline & $\begin{array}{c}\text { Ultra-processed foods } \\
\text { (Expenditure share, } \%)\end{array}$ & $\begin{array}{c}\text { Primary processed foods } \\
\text { (Expenditure share, } \%)\end{array}$ & $\begin{array}{c}\text { Unprocessed foods } \\
\text { (Expenditure share, } \%)\end{array}$ \\
\hline Supermarket & Panel A: Only supermarkets considered & $-0.094 * * *$ \\
Other covariates & $\left(0.051^{* *}\right.$ & $0.043^{*}$ & $(0.027)$ \\
Yes & $(0.021)$ & Yes \\
Hypermarket & Panel B: Multiple food retailers considered & -0.128 \\
& $0.146^{*}$ & -0.018 & $(0.091)$ \\
Supermarket & $(0.071)$ & $(0.095)$ & $-0.143^{*}$ \\
& $0.196^{* * *}$ & -0.053 & $(0.075)$ \\
Convenience store & $(0.052)$ & $(0.075)$ & -0.026 \\
& $0.293^{* * *}$ & $-0.267 * *$ & $(0.097)$ \\
Fast-food restaurant & $(0.091)$ & $(0.110)$ & 0.060 \\
Grocery store & $0.611^{* * *}$ & $-0.671 * * *$ & $(0.168)$ \\
& $(0.109)$ & $(0.091)$ & $-0.174^{* *}$ \\
Traditional market & $0.217^{* * *}$ & -0.043 & $(0.066)$ \\
& $(0.055)$ & $(0.070)$ & 0.058 \\
Roadside market & 0.063 & $-0.122^{*}$ & $(0.070)$ \\
Neighborhood kiosk & $(0.044)$ & $(0.063)$ & $0.122^{*}$ \\
Other covariates & 0.041 & $-0.164^{* *}$ & $(0.063)$ \\
\hline Observations & $(0.054)$ & $(0.061)$ & $-0.173^{*}$
\end{tabular}

Notes: Ordinary least squares estimates are shown with robust standard errors clustered at compound level in parentheses. All types of retailers are represented by the household expenditure share for this retailer. Socioeconomic control variables are included in all models, but are not shown here for brevity. Full estimation results are shown in Table A2.4 in the Supplementary material. * significant at the $10 \%$ level; ** significant at the $5 \%$ level; *** significant at the $1 \%$ level.

be associated with increased risks of obesity and chronic diseases (Monteiro et al., 2010; Popkin, 2017).

The picture becomes more differentiated when also considering the other modern and traditional retailers, as shown in panel B of Table 2.5. The use of supermarkets (and hypermarkets) remains positively associated with the consumption of ultra-processed foods, and the size of the association is even larger than that evident in panel A. An increase in the expenditure share of supermarkets by 1 percentage point increases the expenditure share of ultra-processed foods by about 0.2 percentage points. Modern convenience stores and fastfood restaurants are also associated with higher consumption of ultra-processed foods. Interestingly, however, the same is true for some of the traditional retailers. For traditional grocery stores and neighborhood kiosks the size of the positive association is even somewhat larger than for modern supermarkets and hypermarkets. These results suggest that there is a general shift towards the consumption of ultra-processed foods that cannot be attributed to modern retailers alone. 
As a robustness check, we re-estimated the models in Table 2.5 by using absolute food expenditures for the three processing levels as dependent variables instead of expenditure shares. These alternative results also show that modern retailers as well as traditional grocery stores and kiosks are associated with higher consumption of ultra-processed foods (Table A2.6 in the Supplementary material). Furthermore, we estimated the same models by splitting the sample into poor and non-poor households, using the international poverty line of US\$1.90 a day (World Bank, 2019). Results in Table A2.7 of the Supplementary material suggest that the associations between the use of certain food retailers and the consumption of ultra-processed foods are more pronounced for non-poor than for poor households. This is plausible given that poor households' food choices are more constrained by income limitations. However, as was shown in Table 2.2, poor people also spend more than one-third of their food budget on ultra-processed foods.

\subsubsection{Associations between retailers and food groups}

Table 2.6 shows the associations between the use of different retailers and the consumption of various food groups. In these models, consumption is expressed in terms of the food quantities consumed by the household during the 7-day recall period. The upper part of Table 2.6 (panel A) includes supermarkets as the only retailer variable. The estimates suggest that the use of supermarkets is associated with higher consumption of meat, fish, and dairy products and lower consumption of sugar, sweets, and sweetened beverages.

However, the picture changes somewhat in the lower part of Table 2.6 (panel B), where the other retailers are also included as explanatory variables. The specifications in panel B show that the use of supermarkets and hypermarkets is associated with higher meat, fish, and dairy consumption, but also with higher consumption of sugar, sweets, and sweetened beverages. In addition, the use of modern convenience stores is associated with higher consumption of oils and fats. The higher consumption of animal-source products is likely related to better cooling facilities in modern retail outlets. This is generally positive from a dietary quality and nutrition perspective, as meat, fish, and dairy products are important sources of protein and micronutrients. Table 2.2 showed that the mean consumption of meat and fish in the sample households is not very low. 
Table 2.6: Associations between the use of different retailers and the consumption of selected food groups

\begin{tabular}{|c|c|c|c|c|c|c|c|c|c|}
\hline & \multicolumn{9}{|c|}{ Food quantity (kg/week) } \\
\hline & $\begin{array}{c}\text { Cereals and } \\
\text { Tubers }\end{array}$ & Legumes & Fruits & Vegetables & $\begin{array}{l}\text { Meat and } \\
\text { Fish }\end{array}$ & $\begin{array}{c}\text { Dairy } \\
\text { Products }\end{array}$ & Eggs & $\begin{array}{l}\text { Oils and } \\
\text { Fats }\end{array}$ & $\begin{array}{c}\text { Sugar, } \\
\text { Beverages }\end{array}$ \\
\hline \multicolumn{10}{|c|}{ Panel A: Only supermarkets considered } \\
\hline \multirow[t]{2}{*}{ Supermarket } & -0.003 & -0.001 & -0.005 & -0.001 & $0.015^{* * * *}$ & $0.014 *$ & -0.002 & -0.003 & $-0.010 * * *$ \\
\hline & $(0.006)$ & $(0.005)$ & $(0.004)$ & $(0.009)$ & $(0.006)$ & $(0.007)$ & $(0.001)$ & $(0.002)$ & $(0.004)$ \\
\hline Other covariates & Yes & Yes & Yes & Yes & Yes & Yes & Yes & Yes & Yes \\
\hline \multicolumn{10}{|c|}{ Panel B: Multiple food retailers considered } \\
\hline \multirow[t]{2}{*}{ Hypermarket } & 0.025 & -0.009 & -0.009 & 0.013 & $0.043^{*}$ & $0.053 *$ & 0.007 & 0.009 & $0.040 * * *$ \\
\hline & $(0.031)$ & $(0.019)$ & $(0.018)$ & $(0.020)$ & $(0.023)$ & $(0.029)$ & $(0.006)$ & $(0.005)$ & $(0.008)$ \\
\hline \multirow[t]{2}{*}{ Supermarket } & 0.011 & 0.003 & $-0.031 * *$ & 0.027 & $0.030^{*}$ & $0.055^{* * *}$ & 0.005 & 0.005 & $0.015^{*}$ \\
\hline & $(0.018)$ & $(0.012)$ & $(0.015)$ & $(0.021)$ & $(0.016)$ & $(0.020)$ & $(0.003)$ & $(0.004)$ & $(0.008)$ \\
\hline \multirow[t]{2}{*}{ Convenience store } & $0.058 * *$ & -0.007 & $-0.039 *$ & 0.012 & 0.022 & 0.014 & 0.002 & $0.014 * *$ & 0.020 \\
\hline & $(0.025)$ & $(0.011)$ & $(0.023)$ & $(0.019)$ & $(0.015)$ & $(0.050)$ & $(0.005)$ & $(0.006)$ & $(0.013)$ \\
\hline \multirow[t]{2}{*}{ Fast-food restaurant } & $-0.100 * * *$ & & & & $0.110^{*}$ & $0.132 * *$ & & & $0.105^{* *}$ \\
\hline & $(0.037)$ & & & & $(0.062)$ & $(0.055)$ & & & $(0.049)$ \\
\hline \multirow[t]{2}{*}{ Grocery store } & 0.013 & -0.003 & $-0.030 *$ & 0.016 & 0.026 & $0.063 * *$ & $0.008 * *$ & 0.005 & $0.028 * * *$ \\
\hline & $(0.016)$ & $(0.013)$ & $(0.017)$ & $(0.023)$ & $(0.016)$ & $(0.029)$ & $(0.004)$ & $(0.004)$ & $(0.007)$ \\
\hline \multirow[t]{2}{*}{ Traditional market } & 0.011 & 0.016 & $-0.033^{* *}$ & $0.058 * * *$ & 0.015 & 0.023 & 0.004 & $0.011^{* * *}$ & $0.024 * * *$ \\
\hline & $(0.018)$ & $(0.013)$ & $(0.015)$ & $(0.015)$ & $(0.015)$ & $(0.022)$ & $(0.003)$ & $(0.004)$ & $(0.008)$ \\
\hline \multirow[t]{2}{*}{ Roadside market } & 0.010 & 0.012 & $-0.038 * *$ & $0.038 * *$ & 0.007 & $0.038^{* *}$ & 0.006 & 0.005 & 0.010 \\
\hline & $(0.019)$ & $(0.013)$ & $(0.018)$ & $(0.016)$ & $(0.016)$ & $(0.015)$ & $(0.004)$ & $(0.004)$ & $(0.007)$ \\
\hline \multirow[t]{2}{*}{ Neighborhood kiosk } & 0.030 & -0.013 & -0.014 & -0.008 & -0.010 & $0.057 * *$ & $0.017 * * *$ & 0.007 & $0.027 *$ \\
\hline & $(0.027)$ & $(0.015)$ & $(0.017)$ & $(0.025)$ & $(0.019)$ & $(0.027)$ & $(0.004)$ & $(0.005)$ & $(0.015)$ \\
\hline Other covariates & Yes & Yes & Yes & Yes & Yes & Yes & Yes & Yes & Yes \\
\hline Observations & 475 & 475 & 475 & 475 & 475 & 475 & 475 & 475 & 475 \\
\hline
\end{tabular}

Notes: Tobit estimates are shown with robust standard errors clustered at compound level in parentheses. All types of retailers are represented by the household expenditure share for this retailer. Socioeconomic control variables are included in all models, but are not shown here, for purposes of brevity. Full estimation results are shown in Tables A2.8 and A2.9 in the Supplementary material. * significant at the $10 \%$ level; ** significant at the $5 \%$ level; *** significant at the $1 \%$ level. 
Very high meat consumption levels can also be associated with negative health and environmental externalities (Godfray et al., 2018). However, more sugar, sweets, oils, and fats may contribute to people being overweight and increasing obesity, and therefore, worsen dietary quality and nutrition. In other words, modern retailers seem to be associated with both positive and negative dietary effects.

Strikingly, however, mixed dietary effects are also observed for traditional retailers. On the positive side, the estimates in Table 2.6 suggest that the use of traditional grocery stores and neighborhood kiosks is associated with higher consumption of dairy products and eggs. The use of traditional markets is associated with higher vegetable consumption. On the negative side, the use of grocery stores, traditional markets, and neighborhood kiosks is also associated with higher consumption of sugar, sweets, and sweetened beverages. The use of traditional markets is further associated with higher consumption of oils and fats. These patterns suggest that the retail format and the product ranges offered by different types of retailers do influence consumer food choices and diets, but that there is no clear division between modern and traditional retailers. This finding is in line with the analysis of links between food retailing and processing levels discussed above.

Another noteworthy observation from the estimates in Table 2.6 is that all retailers seem to be associated with lower consumption of fruits; several of these negative associations are statistically significant. This is surprising because consumers actually buy fresh fruits in several of the retail outlets, especially in supermarkets, traditional markets, and roadside markets. However, some of the fruits are also obtained from own production, and we do not include own production as an explanatory variable. Households with own fruit production consume more fruits than households that fully rely on purchases, which can explain the negative associations between all retailers and fruit consumption in Table 2.6. Overall, the consumption of fruits is very low among the sample households from Lusaka City.

In a robustness check of the estimates in Table 2.6 we ran the same models, but used consumption expressed in value terms instead of quantities as dependent variables. These alternative estimates are shown in Table A2.10 in the Supplementary material. The results support the same general conclusions only that the associations with consumption expenditures for sugar, sweets, and sweetened beverages are not statistically significant for any of the modern and traditional retailers. 


\subsection{Conclusions}

Many countries in Africa are experiencing a rapid modernization of their food retail sector, with supermarkets, hypermarkets, modern convenience stores, and fast-food restaurants gaining in importance. These changing food environments, especially in urban areas, may influence consumers' food choices, dietary patterns, and nutrition. Previous research has suggested that the spread of modern retailers may contribute to less healthy diets, higher consumption of ultraprocessed foods, and rising rates of overweight and obesity. However, previous studies did not pay much attention to the question as to which socioeconomic groups use what type of retailers. Furthermore, the existing research on diet and nutrition effects focused primarily on the role of supermarkets, without accounting for the fact that most consumers obtain their foods from various types of retailers. We have added to this research direction by more explicitly analyzing the associations between household socioeconomic status, the use of different types of modern and traditional retailers, and dietary patterns. We have collected and used data from households in Lusaka City in Zambia, one of the places in Southern Africa where food environments have changed dramatically in recent years.

Our results show that almost all households use different types of retailers on a regular basis. Two-thirds of the households use modern and traditional retailers simultaneously. Among the modern retailers, supermarkets account for the largest share of the food purchases, followed by modern convenience stores and hypermarkets. Overall, in Lusaka City, modern retailers account for $42 \%$ of the household food expenditures on average, although with notable differences between poor and rich households. Modern retailers account for $20 \%$ and $63 \%$ of total food expenditures in the lowest and highest income tercile, respectively. Income is also an important predictor of the use of modern retailers after controlling for other socioeconomic variables. Other variables that increase the likelihood of using modern retailers are education, car ownership, having an office job, and female household heads. Supermarkets and hypermarkets, in particular, offer a large variety of products, which consumers perceive as safe and of high quality. Supermarkets and hypermarkets also have longer and more reliable opening hours than most traditional retailers. All of these factors make supermarkets and hypermarkets attractive shopping places especially for better-off households with high opportunity costs of time. 
The regression analysis also shows that using supermarkets is associated with a higher consumption of ultra-processed foods and a lower consumption of unprocessed foods, also after controlling for income and other socioeconomic variables. This is in line with earlier research on the dietary effects of supermarkets (Asfaw, 2011; Rischke et al., 2015; Kimenju et al., 2015; Demmler et al., 2018; Rupa et al., 2019). From a nutrition and health perspective, these dietary trends are undesirable, as high consumption of ultra-processed foods is associated with increased risks of obesity and chronic diseases (Monteiro et al., 2010; Beatty et al., 2014; Steyn and Mchiza, 2014; Popkin, 2017). However, unlike earlier studies, we also analyzed the role of other retailers and found that especially the use of traditional grocery stores and neighborhood kiosks is also associated with higher consumption of ultra-processed foods. These results suggest that there is a general shift towards the consumption of ultra-processed foods that cannot be attributed to modern retailers alone.

We also analyzed the consumption of different food groups and found that the use of modern retailers is associated with higher consumption of certain unhealthy food groups (sugar, sweets, oils, fats), but also with higher consumption of certain healthy food groups (meat, fish, dairy products). At the same time, the use of some of the traditional retailers - such as grocery stores, traditional markets, and kiosks - is also associated with higher consumption of unhealthy food groups.

Many countries in Africa are experiencing a nutrition transition with both positive and negative implications. On the positive side, the consumption of some nutritious foods is increasing. On the negative side, the consumption of sugar, fat, and salt is increasing as well. Changing food environments seem to influence and support these dietary trends and should, therefore, also be seen as potential entry points for public regulations and policies to support more healthy diets. Policy options to consider are regulations related to the advertisement and promotion of healthy and unhealthy foods and their strategic placement within shops. For instance, in studies referring to industrialized countries, Glanz et al. (2012) and Payne and Niculescu (2018) showed that changes in the placement of fruits and vegetables can positively influence consumer choices. Related regulations could also be relevant for countries in Africa. In urban Zambia, the consumption of fresh fruits is particularly low; policies to increase fruit consumption levels would be useful. Beyond advertisement, awareness campaigns, and nudges, taxes and subsidies 
could also be options to promote healthy diets. A detailed discussion of policy approaches is beyond the scope of this article. In any case, our results emphasize that modern retailers are not the only drivers of dietary transitions, so that a focus on regulating modern retailers alone would be insufficient to promote healthy eating.

In closing, three limitations of our research should briefly be discussed. First, we used processing level categories, which could not sufficiently classify the degree of healthfulness of a specific food. Moreover, the three categories (ultra-processed, primary processed and unprocessed foods) could not properly account for the overlap in nutritional attributes for some food products. Second, we used observational data and could not control for the endogeneity of households' decisions about which retailers to use. Therefore, our results are interpreted only in terms of associations, not as causal effects. Proper identification is difficult with observational data, but longer-term studies with panel data may possibly help. Third, results from Lusaka City in Zambia are not necessarily representative for other parts of Africa. Follow-up research in different geographical contexts would be interesting to further broaden the knowledge base. 


\section{Appendix A2}

\section{Table A2.1: Food processing levels by food groups and items}

\begin{tabular}{|c|c|c|}
\hline Processing level & Food group & Food items (Examples) \\
\hline \multirow{5}{*}{ Unprocessed foods } & Cereals and tubers & Maize (dry/green), cassava, Irish potato, sweet potato, yams \\
\hline & Eggs and milk & Eggs, fresh whole milk \\
\hline & Fruits & $\begin{array}{l}\text { Apples, avocado, banana (ripe/boiled), guava, mango, pawpaw, pineapple, } \\
\text { pumpkin, orange/tangerine, sugar plum, watermelon }\end{array}$ \\
\hline & Legumes & $\begin{array}{l}\text { Bean (fresh/dry), cowpea (fresh/dry), groundnut (fresh/dry), pigeonpea } \\
\text { (fresh/dry), soybean, velvet bean }\end{array}$ \\
\hline & Vegetables & $\begin{array}{l}\text { Bean leaves, blackjack, cabbage, carrot, cassava leaves, cowpea leaves, } \\
\text { cucumber, eggplant, garlic, greengram, lettuce, mushroom } \\
\text { (cultivated/wild), okra, onion, pepper, pumpkin leaves, } \\
\text { rape/mustard/chinese, tomato }\end{array}$ \\
\hline \multirow{3}{*}{ Primary processed foods } & Drinks and snacks & Bottled/clear beer, bottled water, roasted cashew/macadamia nuts \\
\hline & Meat and fish & $\begin{array}{l}\text { Beef, bush/game meat, chicken, duck, turkey, goat meat, sheep meat, pork, } \\
\text { fish (fresh/frozen/dried) }\end{array}$ \\
\hline & Cereals & Rice, millet, oats, sorghum \\
\hline \multirow{7}{*}{ Ultra-processed foods } & Bread and pasta & Bread, buns, pasta, instant noodles \\
\hline & Cereals and tubers & Maize flour, cornflakes, porridge mix, wheat flour, cassava flour \\
\hline & Dairy products & Cheese, milk, yoghurt \\
\hline & Oils and fats & Butter/margarine, coconut oil, cooking oil/fat \\
\hline & Meat and fish & Sausage (beef/chicken/pork), soya meat, canned meat and fish \\
\hline & Miscellaneous & $\begin{array}{l}\text { Canned foods, mandazi, mixed fruits/salads, pizza, samosa, ready-made } \\
\text { foods/dishes }\end{array}$ \\
\hline & $\begin{array}{l}\text { Sugar, sweetened drinks and } \\
\text { snacks }\end{array}$ & $\begin{array}{l}\text { Soft drinks, sweetened fruit juices, wine, jam, tomato sauce, salt, sugar, } \\
\text { biscuits/cookies, cake, chips, chocolate, crisps, puffed salted corn chips, } \\
\text { popcorn, salted nuts }\end{array}$ \\
\hline
\end{tabular}

Note: The same classifications of foods were also used by Demmler et al. (2018). 
Table A2.2: Additional descriptive statistics

\begin{tabular}{|c|c|c|c|c|}
\hline & \multirow{2}{*}{ Full sample } & \multicolumn{3}{|c|}{ By income tercile } \\
\hline & & Lowest & Middle & Highest \\
\hline \multicolumn{5}{|l|}{ Socioeconomic characteristics } \\
\hline \multirow[t]{2}{*}{ Age of household head (years) } & 43.83 & 45.13 & 41.98 & 44.40 \\
\hline & $(12.86)$ & $(13.67)$ & (12.68) & $(12.02)$ \\
\hline \multirow[t]{2}{*}{ Adolescent in household (dummy) } & 0.47 & 0.50 & 0.49 & 0.43 \\
\hline & $(0.50)$ & $(0.50)$ & $(0.50)$ & $(0.50)$ \\
\hline \multirow[t]{2}{*}{ Child in household (dummy) } & 0.59 & 0.71 & 0.53 & 0.54 \\
\hline & $(0.49)$ & $(0.45)$ & $(0.50)$ & $(0.50)$ \\
\hline \multirow[t]{2}{*}{ Bemba ethnicity (dummy) } & 0.29 & 0.28 & 0.24 & 0.36 \\
\hline & $(0.45)$ & $(0.45)$ & $(0.43)$ & $(0.48)$ \\
\hline \multirow[t]{2}{*}{ Tonga ethnicity (dummy) } & 0.19 & 0.15 & 0.21 & 0.21 \\
\hline & $(0.39)$ & $(0.36)$ & $(0.41)$ & $(0.41)$ \\
\hline \multirow[t]{2}{*}{ Protestant religion (dummy) } & 0.42 & 0.42 & 0.46 & 0.38 \\
\hline & $(0.49)$ & $(0.49)$ & $(0.50)$ & $(0.49)$ \\
\hline \multirow[t]{2}{*}{ Catholic religion (dummy) } & 0.26 & 0.31 & 0.19 & 0.29 \\
\hline & $(0.44)$ & $(0.47)$ & $(0.39)$ & $(0.45)$ \\
\hline \multicolumn{5}{|l|}{ Food expenditures } \\
\hline \multirow[t]{2}{*}{ Cereals and tubers (ZMW/week) } & 106.41 & 87.37 & 108.25 & 123.94 \\
\hline & $(57.02)$ & $(49.60)$ & $(55.01)$ & $(60.40)$ \\
\hline \multirow[t]{2}{*}{ Legumes (ZMW/week) } & 30.15 & 30.84 & 32.36 & 27.16 \\
\hline & $(43.99)$ & $(43.52)$ & $(49.17)$ & $(38.63)$ \\
\hline \multirow[t]{2}{*}{ Fruits (ZMW/week) } & 7.88 & 7.23 & 6.75 & 9.70 \\
\hline & (20.64) & (20.94) & $(17.37)$ & $(23.25)$ \\
\hline \multirow[t]{2}{*}{ Vegetables (ZMW/week) } & 59.63 & 57.99 & 64.98 & 55.82 \\
\hline & $(44.19)$ & $(39.59)$ & $(47.19)$ & $(45.21)$ \\
\hline \multirow[t]{2}{*}{ Meat and fish (ZMW/week) } & 172.84 & 126.04 & 178.54 & 214.69 \\
\hline & $(116.61)$ & (100.16) & $(107.59)$ & $(124.26)$ \\
\hline \multirow[t]{2}{*}{ Dairy products and eggs (ZMW/week) } & 23.53 & 14.45 & 18.54 & 37.90 \\
\hline & $(33.24)$ & $(18.70)$ & $(25.32)$ & $(45.25)$ \\
\hline \multirow[t]{2}{*}{ Oils and fats (ZMW/week) } & 9.82 & 9.14 & 10.28 & 10.05 \\
\hline & $(9.47)$ & $(8.65)$ & $(9.09)$ & $(10.61)$ \\
\hline \multirow[t]{2}{*}{ Sugar, sweetened beverages (ZMW/week) } & 33.86 & 27.55 & 32.23 & 41.96 \\
\hline & $(50.67)$ & $(42.59)$ & $(43.95)$ & $(62.54)$ \\
\hline Observations & 475 & 159 & 160 & 156 \\
\hline
\end{tabular}

Notes: Mean values are shown with standard deviations in parentheses. ZMW, Zambia Kwacha (local currency). The average exchange rate was ZMW 9.87 = US\$ 1 in mid-2018. 


\section{Table A2.3: Correlation matrix from Multivariate Probit Model}

\begin{tabular}{|c|c|c|c|c|c|c|c|c|}
\hline & \multicolumn{4}{|c|}{ Modern retailer } & \multicolumn{4}{|c|}{ Traditional retailer } \\
\hline & HM & SM & $\mathrm{CS}$ & $\mathrm{FF}$ & GS & $\mathrm{TM}$ & $\mathrm{RM}$ & NK \\
\hline $\begin{array}{l}\text { Hypermarket } \\
\text { (HM) }\end{array}$ & 1.000 & & & & & & & \\
\hline \multirow{2}{*}{$\begin{array}{l}\text { Supermarket } \\
\text { (SM) }\end{array}$} & 0.161 & 1.000 & & & & & & \\
\hline & $(0.122)$ & & & & & & & \\
\hline \multirow{2}{*}{$\begin{array}{l}\text { Convenience } \\
\text { store (CS) }\end{array}$} & $0.252^{* *}$ & 0.149 & 1.000 & & & & & \\
\hline & $(0.114)$ & $(0.099)$ & & & & & & \\
\hline \multirow{2}{*}{$\begin{array}{l}\text { Fast-food } \\
\text { restaurant (FF) }\end{array}$} & -0.088 & -0.047 & 0.198 & 1.000 & & & & \\
\hline & $(0.236)$ & $(0.220)$ & $(0.205)$ & & & & & \\
\hline \multirow{2}{*}{$\begin{array}{l}\text { Grocery store } \\
\text { (GS) }\end{array}$} & -0.098 & $-0.304 * * *$ & 0.009 & $0.388 * * *$ & 1.000 & & & \\
\hline & $(0.108)$ & $(0.073)$ & $(0.090)$ & $(0.122)$ & & & & \\
\hline \multirow{2}{*}{$\begin{array}{l}\text { Traditional } \\
\text { market (TM) }\end{array}$} & 0.074 & $-0.164^{*}$ & 0.064 & -0.046 & 0.022 & 1.000 & & \\
\hline & $(0.108)$ & $(0.084)$ & $(0.091)$ & $(0.127)$ & $(0.080)$ & & & \\
\hline \multirow{2}{*}{$\begin{array}{l}\text { Roadside market } \\
\text { (RM) }\end{array}$} & 0.060 & -0.040 & $0.163^{*}$ & $0.285^{* *}$ & $0.249 * * *$ & $-0.282 * * *$ & 1.000 & \\
\hline & $(0.105)$ & $(0.086)$ & $(0.091)$ & $(0.124)$ & $(0.076)$ & $(0.081)$ & & \\
\hline \multirow{2}{*}{$\begin{array}{l}\text { Neighborhood } \\
\text { kiosk (NK) }\end{array}$} & -0.003 & $-0.145^{*}$ & -0.086 & 0.137 & $0.222 * * *$ & -0.124 & -0.026 & 1.000 \\
\hline & $(0.117)$ & $(0.086)$ & $(0.096)$ & $(0.114)$ & $(0.081)$ & $(0.086)$ & $(0.083)$ & \\
\hline
\end{tabular}

Notes: Correlation coefficients are shown with standard errors in parentheses. The likelihood ratio test of zero correlation between the error terms is rejected at the $1 \%$ level; $\chi^{2}(28)=85$. * significant at the $10 \%$ level; ** significant at the $5 \%$ level; *** significant at the $1 \%$ level. 
Table A2.4: Associations between the use of retailers and food processing levels (full model results for Table 2.5)

\begin{tabular}{|c|c|c|c|c|c|c|}
\hline & \multicolumn{3}{|c|}{ Only supermarkets considered: Panel A } & \multicolumn{3}{|c|}{ Multiple food retailers considered: Panel B } \\
\hline & $\begin{array}{l}\text { Ultra-processed } \\
\text { foods }\end{array}$ & $\begin{array}{c}\text { Primary } \\
\text { processed foods }\end{array}$ & $\begin{array}{l}\text { Unprocessed } \\
\text { foods }\end{array}$ & $\begin{array}{l}\text { Ultra-processed } \\
\text { foods }\end{array}$ & $\begin{array}{c}\text { Primary } \\
\text { processed foods }\end{array}$ & $\begin{array}{c}\text { Unprocessed } \\
\text { foods }\end{array}$ \\
\hline & (1) & (2) & (3) & $(1)$ & $(2)$ & (3) \\
\hline \multirow[t]{2}{*}{ Hypermarket } & & & & $0.146^{*}$ & -0.018 & -0.128 \\
\hline & & & & $(0.071)$ & $(0.095)$ & $(0.091)$ \\
\hline \multirow[t]{2}{*}{ Supermarket } & $0.051^{* *}$ & $0.043 *$ & $-0.094 * * *$ & $0.196 * * *$ & -0.053 & $-0.143^{*}$ \\
\hline & $(0.022)$ & $(0.021)$ & $(0.027)$ & $(0.052)$ & $(0.075)$ & $(0.075)$ \\
\hline \multirow[t]{2}{*}{ Convenience store } & & & & $0.293 * * *$ & $-0.267 * *$ & -0.026 \\
\hline & & & & $(0.091)$ & $(0.110)$ & $(0.097)$ \\
\hline \multirow[t]{2}{*}{ Fast-food restaurant } & & & & $0.611^{* * *}$ & $-0.671^{* * *}$ & 0.060 \\
\hline & & & & $(0.109)$ & $(0.091)$ & $(0.168)$ \\
\hline \multirow[t]{2}{*}{ Grocery store } & & & & $0.217 * * *$ & -0.043 & $-0.174 * *$ \\
\hline & & & & $(0.055)$ & $(0.070)$ & $(0.066)$ \\
\hline \multirow[t]{2}{*}{ Traditional market } & & & & 0.063 & $-0.122 *$ & 0.058 \\
\hline & & & & $(0.044)$ & $(0.063)$ & $(0.070)$ \\
\hline \multirow[t]{2}{*}{ Roadside market } & & & & 0.041 & $-0.164 * *$ & $0.122 *$ \\
\hline & & & & $(0.054)$ & $(0.061)$ & $(0.063)$ \\
\hline \multirow[t]{2}{*}{ Neighborhood kiosk } & & & & $0.274 * * *$ & -0.101 & $-0.173^{*}$ \\
\hline & & & & $(0.079)$ & $(0.093)$ & $(0.098)$ \\
\hline \multirow[t]{2}{*}{ Male } & -0.174 & -0.374 & 0.548 & -0.648 & -0.313 & 0.961 \\
\hline & (1.404) & (1.996) & (1.911) & $(1.221)$ & (1.939) & $(1.646)$ \\
\hline \multirow[t]{2}{*}{ Age } & 0.001 & -0.057 & 0.056 & 0.015 & -0.071 & $0.056^{*}$ \\
\hline & $(0.050)$ & $(0.039)$ & $(0.050)$ & $(0.039)$ & $(0.043)$ & $(0.027)$ \\
\hline \multirow[t]{2}{*}{ Household size } & $0.707 *$ & $-1.254 * * *$ & 0.547 & $0.875^{* *}$ & $-1.063 * *$ & 0.188 \\
\hline & $(0.363)$ & $(0.404)$ & $(0.445)$ & $(0.333)$ & $(0.433)$ & $(0.304)$ \\
\hline \multirow[t]{2}{*}{ Education } & $-0.550^{* * *}$ & $0.700^{* * *}$ & -0.150 & $-0.601 * * *$ & $0.673 * * *$ & -0.072 \\
\hline & $(0.122)$ & $(0.214)$ & $(0.168)$ & $(0.126)$ & $(0.198)$ & $(0.182)$ \\
\hline \multirow[t]{2}{*}{ Income (log) } & 0.159 & $1.389 *$ & $-1.548^{* *}$ & -0.440 & $1.297 *$ & -0.857 \\
\hline & $(0.423)$ & $(0.714)$ & $(0.621)$ & $(0.458)$ & $(0.712)$ & $(0.599)$ \\
\hline \multirow[t]{2}{*}{ Chewa } & 0.089 & 2.757 & -2.846 & -0.241 & 2.327 & -2.086 \\
\hline & (1.643) & $(2.022)$ & $(2.565)$ & (1.589) & (2.188) & $(2.212)$ \\
\hline \multirow[t]{2}{*}{ Tonga } & 0.544 & 1.406 & -1.950 & -0.001 & 1.319 & -1.318 \\
\hline & (1.608) & $(2.057)$ & $(1.553)$ & (1.698) & (2.208) & $(1.581)$ \\
\hline \multirow[t]{2}{*}{ Catholic } & -0.977 & -0.171 & 1.148 & -1.150 & -0.697 & $1.847 * *$ \\
\hline & $(2.111)$ & $(1.847)$ & $(0.963)$ & (2.064) & $(1.968)$ & $(0.713)$ \\
\hline \multirow[t]{2}{*}{ Seventh Day Adventist } & -2.916 & 0.252 & $2.664^{*}$ & -2.075 & 0.570 & 1.505 \\
\hline & $(1.719)$ & $(1.770)$ & $(1.441)$ & $(1.566)$ & $(1.985)$ & $(1.413)$ \\
\hline \multirow[t]{2}{*}{ Constant } & $35.601 * * *$ & $22.579 * * *$ & $41.820^{* * *}$ & $29.124 * * *$ & $33.773 * * *$ & $37.103^{* * *}$ \\
\hline & $(5.417)$ & $(5.688)$ & (5.499) & $(7.932)$ & $(8.944)$ & $(9.491)$ \\
\hline R-squared & 0.035 & 0.122 & 0.146 & 0.116 & 0.149 & 0.256 \\
\hline Observations & 475 & 475 & 475 & 475 & 475 & 475 \\
\hline
\end{tabular}

Notes: Ordinary least squares estimates are shown with robust standard errors clustered at compound level in parentheses. All types of retailers are represented by the household expenditure share for this retailer. Bemba and Protestant are used as a reference group for ethnicity - Chewa and Tonga, and religion status - Catholic and Seventh Day Adventist, respectively. * significant at the $10 \%$ level; ** significant at the 5\% level; *** significant at the $1 \%$ level. 


\section{Table A2.5: Associations between supermarket use and food processing levels (Seemingly}

\section{unrelated regressions)}

\begin{tabular}{|c|c|c|c|}
\hline & $\begin{array}{l}\text { Ultra-processed foods } \\
\text { (Expenditure share) }\end{array}$ & $\begin{array}{l}\text { Primary processed foods } \\
\text { (Expenditure share) }\end{array}$ & $\begin{array}{l}\text { Unprocessed foods } \\
\text { (Expenditure share) }\end{array}$ \\
\hline \multirow[t]{2}{*}{ Supermarket } & $0.051 * *$ & 0.043 & $-0.094 * * *$ \\
\hline & $(0.023)$ & $(0.028)$ & $(0.023)$ \\
\hline \multirow{2}{*}{ Male } & -0.174 & -0.374 & 0.548 \\
\hline & (1.318) & (1.593) & (1.299) \\
\hline \multirow[t]{2}{*}{ Age } & 0.001 & -0.057 & 0.056 \\
\hline & $(0.051)$ & $(0.061)$ & $(0.050)$ \\
\hline \multirow[t]{2}{*}{ Household size } & $0.707 *$ & $-1.254 * *$ & 0.547 \\
\hline & $(0.412)$ & $(0.497)$ & $(0.406)$ \\
\hline \multirow[t]{2}{*}{ Education } & $-0.550^{* *}$ & $0.700 * * *$ & -0.150 \\
\hline & $(0.219)$ & $(0.264)$ & $(0.216)$ \\
\hline \multirow[t]{2}{*}{ Income $(\log )$} & 0.159 & $1.389^{*}$ & $-1.548^{* *}$ \\
\hline & $(0.645)$ & $(0.780)$ & $(0.636)$ \\
\hline \multirow[t]{2}{*}{ Chewa } & 0.089 & 2.757 & -2.846 \\
\hline & (1.932) & $(2.335)$ & (1.905) \\
\hline \multirow[t]{2}{*}{ Tonga } & 0.544 & 1.406 & -1.950 \\
\hline & (1.756) & $(2.122)$ & (1.731) \\
\hline \multirow[t]{2}{*}{ Catholic } & -0.977 & -0.171 & 1.148 \\
\hline & (1.474) & (1.781) & (1.453) \\
\hline \multirow[t]{2}{*}{ Seventh Day Adventist } & -2.916 & 0.252 & 2.664 \\
\hline & $(1.865)$ & $(2.254)$ & (1.839) \\
\hline \multirow[t]{2}{*}{ Constant } & $35.601^{* * * *}$ & $22.579^{* * *}$ & $41.820^{* * *}$ \\
\hline & (6.492) & $(7.845)$ & $(6.401)$ \\
\hline Observations & 475 & 475 & 475 \\
\hline
\end{tabular}

Notes: Seemingly unrelated regression estimates are shown with standard errors in parentheses. Supermarkets are represented by the household expenditure share for this retailer. * significant at the $10 \%$ level; ** significant at the 5\% level; *** significant at the $1 \%$ level. 
Table A2.6: Associations between the use of different retailers and food processing levels (absolute expenditures)

\begin{tabular}{|c|c|c|c|}
\hline & $\begin{array}{l}\text { Ultra-processed foods } \\
\text { (Expenditures, log) }\end{array}$ & $\begin{array}{l}\text { Primary processed foods } \\
\text { (Expenditures, log) }\end{array}$ & $\begin{array}{l}\text { Unprocessed foods } \\
\text { (Expenditures, log) }\end{array}$ \\
\hline \multicolumn{4}{|c|}{ Panel A: Supermarkets only } \\
\hline \multirow[t]{2}{*}{ Supermarket } & 0.002 & 0.002 & $-0.004 *$ \\
\hline & $(0.001)$ & $(0.001)$ & $(0.002)$ \\
\hline Other covariates & Yes & Yes & Yes \\
\hline \multicolumn{4}{|c|}{ Panel B: Multiple food retailers considered } \\
\hline \multirow[t]{2}{*}{ Hypermarket } & $0.012 * * *$ & $0.007 *$ & 0.006 \\
\hline & $(0.004)$ & $(0.004)$ & $(0.006)$ \\
\hline \multirow[t]{2}{*}{ Supermarket } & $0.009 * *$ & 0.003 & 0.0005 \\
\hline & $(0.003)$ & $(0.003)$ & $(0.006)$ \\
\hline \multirow[t]{2}{*}{ Convenience store } & $0.014 * * *$ & -0.003 & 0.009 \\
\hline & $(0.005)$ & $(0.003)$ & $(0.006)$ \\
\hline \multirow[t]{2}{*}{ Fast-food restaurant } & $0.041 * * *$ & 0.0005 & $0.029 * * *$ \\
\hline & $(0.006)$ & $(0.012)$ & $(0.006)$ \\
\hline \multirow[t]{2}{*}{ Grocery store } & $0.009 * *$ & 0.001 & -0.002 \\
\hline & $(0.003)$ & $(0.003)$ & $(0.006)$ \\
\hline \multirow[t]{2}{*}{ Traditional market } & $0.006^{*}$ & 0.002 & $0.011 * *$ \\
\hline & $(0.003)$ & $(0.003)$ & $(0.005)$ \\
\hline \multirow[t]{2}{*}{ Roadside market } & 0.003 & -0.002 & $0.010^{* *}$ \\
\hline & $(0.003)$ & $(0.003)$ & $(0.004)$ \\
\hline \multirow[t]{2}{*}{ Neighborhood kiosk } & $0.010^{* *}$ & 0.0002 & -0.004 \\
\hline & $(0.004)$ & $(0.003)$ & $(0.006)$ \\
\hline Other covariates & Yes & Yes & Yes \\
\hline Observations & 475 & 469 & 471 \\
\hline
\end{tabular}

Notes: Ordinary least squares estimates are shown with robust standard errors clustered at compound level in parentheses. All types of retailers are represented by the household expenditure share for this retailer. The same socioeconomic control variables are included as in Table A2.4. * significant at the $10 \%$ level; ** significant at the $5 \%$ level; *** significant at the $1 \%$ level. 
Table A2.7: Associations between the use of different retailers and food processing levels

\section{(by poverty status)}

\begin{tabular}{|c|c|c|c|c|}
\hline & \multicolumn{2}{|c|}{ Poor households } & \multicolumn{2}{|c|}{ Non-poor households } \\
\hline & $\begin{array}{l}\text { Ultra-processed } \\
\text { (Exp. share, \%) }\end{array}$ & $\begin{array}{l}\text { Unprocessed (Exp. } \\
\text { share, \%) }\end{array}$ & $\begin{array}{l}\text { Ultra-Processed (Exp. } \\
\text { share, \%) }\end{array}$ & $\begin{array}{c}\text { Unprocessed } \\
\text { (Exp. share, \%) }\end{array}$ \\
\hline \multicolumn{5}{|c|}{ Panel A: Only supermarkets considered } \\
\hline \multirow[t]{2}{*}{ Supermarket only } & 0.031 & -0.058 & $0.057 * *$ & $-0.111^{* * *}$ \\
\hline & $(0.050)$ & $(0.087)$ & $(0.026)$ & $(0.025)$ \\
\hline Other covariates & Yes & Yes & Yes & Yes \\
\hline \multicolumn{5}{|c|}{ Panel B: Multiple food retailers considered } \\
\hline \multirow[t]{2}{*}{ Hypermarket } & & & $0.165^{* *}$ & -0.118 \\
\hline & & & $(0.060)$ & $(0.087)$ \\
\hline \multirow[t]{2}{*}{ Supermarket } & 0.035 & -0.165 & $0.231 * * *$ & -0.144 \\
\hline & $(0.128)$ & $(0.205)$ & $(0.047)$ & $(0.095)$ \\
\hline \multirow[t]{2}{*}{ Convenience store } & $0.274 *$ & 0.114 & $0.329^{* *}$ & -0.076 \\
\hline & $(0.130)$ & $(0.187)$ & $(0.111)$ & $(0.125)$ \\
\hline \multirow[t]{2}{*}{ Fast-food restaurant } & -0.627 & -1.055 & $0.679 * * *$ & 0.071 \\
\hline & $(0.853)$ & $(1.003)$ & $(0.081)$ & $(0.219)$ \\
\hline \multirow[t]{2}{*}{ Grocery store } & 0.009 & -0.165 & $0.269^{* * *}$ & $-0.225^{*}$ \\
\hline & $(0.118)$ & $(0.163)$ & $(0.052)$ & $(0.106)$ \\
\hline \multirow[t]{2}{*}{ Traditional market } & -0.029 & -0.022 & 0.064 & 0.106 \\
\hline & $(0.098)$ & $(0.151)$ & $(0.048)$ & $(0.084)$ \\
\hline \multirow[t]{2}{*}{ Roadside market } & -0.073 & 0.020 & 0.056 & $0.158^{*}$ \\
\hline & $(0.075)$ & $(0.147)$ & $(0.067)$ & $(0.084)$ \\
\hline \multirow[t]{2}{*}{ Neighborhood kiosk } & 0.040 & $-0.375 * *$ & $0.358^{* * *}$ & -0.067 \\
\hline & $(0.147)$ & $(0.165)$ & $(0.053)$ & $(0.123)$ \\
\hline Other covariates & Yes & Yes & Yes & Yes \\
\hline Observations & 126 & 126 & 349 & 349 \\
\hline
\end{tabular}

Notes: Ordinary least squares estimates are shown with robust standard errors clustered at compound level in parentheses. Poor households are those with less than US\$1.90 per capita and day in purchasing power parity terms (World Bank, 2019). All types of retailers are represented by the household expenditure share for this retailer. For poor households, hypermarkets were dropped due to perfect collinearity. The same socioeconomic control variables are included as in Table A2.4. * significant at the $10 \%$ level; ** significant at the 5\% level; *** significant at the $1 \%$ level. 
Table A2.8: Associations between the use of different retailers and the consumption of food groups (full model results for Table 2.6, supermarkets only)

\begin{tabular}{|c|c|c|c|c|c|c|c|c|c|}
\hline & \multicolumn{9}{|c|}{ Household food consumption (kg/week) } \\
\hline & $\begin{array}{l}\text { Cereals and } \\
\text { Tubers }\end{array}$ & Legumes & Fruits & Vegetables & Meat and Fish & $\begin{array}{l}\text { Dairy } \\
\text { Products }\end{array}$ & Eggs & $\begin{array}{l}\text { Oils and } \\
\text { Fats }\end{array}$ & $\begin{array}{c}\text { Sugar, } \\
\text { Beverages }\end{array}$ \\
\hline \multirow[t]{2}{*}{ Supermarket } & -0.003 & -0.001 & -0.005 & -0.001 & $0.015^{* * *}$ & $0.014 *$ & -0.002 & -0.003 & $-0.010^{* * *}$ \\
\hline & $(0.006)$ & $(0.005)$ & $(0.004)$ & $(0.009)$ & $(0.006)$ & $(0.007)$ & $(0.001)$ & $(0.002)$ & $(0.004)$ \\
\hline \multirow[t]{2}{*}{ Male } & 0.281 & $0.646^{* * *}$ & -0.031 & 0.627 & 0.361 & -0.072 & 0.122 & $0.397 * * *$ & 0.150 \\
\hline & $(0.471)$ & $(0.160)$ & $(0.338)$ & $(0.483)$ & $(0.245)$ & $(0.552)$ & $(0.083)$ & $(0.085)$ & $(0.167)$ \\
\hline \multirow[t]{2}{*}{ Age } & 0.026 & 0.006 & 0.006 & -0.007 & -0.003 & 0.011 & 0.002 & $0.008^{* * *}$ & $0.019^{* * *}$ \\
\hline & $(0.021)$ & $(0.007)$ & $(0.012)$ & $(0.014)$ & $(0.008)$ & $(0.019)$ & $(0.004)$ & $(0.003)$ & $(0.006)$ \\
\hline \multirow[t]{2}{*}{ Household size } & $0.907 * * *$ & $0.233^{* * * *}$ & -0.074 & $0.384 * * *$ & $0.145^{* *}$ & 0.278 & -0.017 & $0.052^{*}$ & 0.121 \\
\hline & $(0.137)$ & $(0.078)$ & $(0.072)$ & $(0.112)$ & $(0.072)$ & $(0.211)$ & $(0.020)$ & $(0.029)$ & $(0.098)$ \\
\hline \multirow[t]{2}{*}{ Education } & 0.042 & 0.032 & $0.067 *$ & -0.038 & $0.150^{* * * *}$ & 0.121 & $0.050 * *$ & $-0.017^{*}$ & $0.103^{* * *}$ \\
\hline & $(0.048)$ & $(0.031)$ & $(0.037)$ & $(0.065)$ & $(0.043)$ & $(0.097)$ & $(0.020)$ & $(0.010)$ & $(0.022)$ \\
\hline \multirow[t]{2}{*}{ Income (log) } & $0.441 * *$ & -0.203 & 0.142 & -0.205 & $0.445^{* *}$ & $0.844 * * *$ & $0.121 * * *$ & $0.083^{* *}$ & 0.217 \\
\hline & $(0.203)$ & $(0.139)$ & $(0.133)$ & $(0.210)$ & $(0.174)$ & $(0.286)$ & $(0.044)$ & $(0.038)$ & $(0.134)$ \\
\hline \multirow[t]{2}{*}{ Chewa } & $0.933 *$ & 0.589 & 0.128 & -0.422 & $0.790^{* * *}$ & -0.619 & 0.065 & $0.468 * * *$ & 0.517 \\
\hline & $(0.555)$ & $(0.361)$ & $(0.328)$ & $(0.719)$ & $(0.309)$ & $(0.707)$ & $(0.126)$ & $(0.098)$ & $(0.360)$ \\
\hline \multirow[t]{2}{*}{ Tonga } & 0.269 & $0.438^{* *}$ & -0.402 & $1.042^{* *}$ & $0.581 *$ & 0.344 & 0.037 & $0.249^{* * *}$ & -0.095 \\
\hline & $(0.518)$ & $(0.197)$ & $(0.296)$ & $(0.497)$ & $(0.327)$ & $(0.513)$ & $(0.159)$ & $(0.067)$ & $(0.219)$ \\
\hline \multirow[t]{2}{*}{ Catholic } & 0.107 & 0.172 & 0.309 & 0.545 & -0.327 & -0.288 & 0.121 & -0.030 & 0.007 \\
\hline & $(0.386)$ & $(0.252)$ & $(0.305)$ & $(0.554)$ & $(0.345)$ & $(0.344)$ & $(0.087)$ & $(0.086)$ & $(0.205)$ \\
\hline \multirow[t]{2}{*}{$\begin{array}{l}\text { Seventh Day } \\
\text { Adventist }\end{array}$} & $0.964 * *$ & 0.367 & $0.632 * * * *$ & 0.704 & -0.465 & 0.363 & -0.114 & $0.165^{* *}$ & -0.295 \\
\hline & $(0.393)$ & $(0.272)$ & $(0.232)$ & $(0.588)$ & $(0.444)$ & $(0.512)$ & $(0.132)$ & $(0.077)$ & $(0.218)$ \\
\hline \multirow[t]{2}{*}{ Constant } & -0.035 & 0.813 & $-3.536^{*}$ & $4.512^{* *}$ & $-3.650^{* *}$ & $-15.110^{* * *}$ & $-1.964 * * *$ & $-1.043^{* *}$ & $-3.487^{* *}$ \\
\hline & $(2.355)$ & (1.145) & $(2.017)$ & $(2.238)$ & (1.496) & (4.112) & $(0.558)$ & $(0.428)$ & $(1.494)$ \\
\hline Pseudo-R-squared & 0.060 & 0.027 & 0.014 & 0.011 & 0.073 & 0.081 & 0.053 & 0.068 & 0.024 \\
\hline Observations & 475 & 475 & 475 & 475 & 475 & 475 & 475 & 475 & 475 \\
\hline
\end{tabular}

Notes: Tobit estimates are shown with robust standard errors clustered at compound level in parentheses. All types of retailers are represented by the household expenditure share for this retailer. * significant at the $10 \%$ level; ** significant at the $5 \%$ level; *** significant at the $1 \%$ level. 
Table A2.9: Associations between the use of different retailers and the consumption of food groups (full model results for Table 2.6, all retailers)

\begin{tabular}{|c|c|c|c|c|c|c|c|c|c|}
\hline & \multicolumn{9}{|c|}{ Household food consumption (kg/week) } \\
\hline & $\begin{array}{l}\text { Cereals and } \\
\text { Tubers }\end{array}$ & Legumes & Fruits & Vegetables & Meat and Fish & Dairy Products & Eggs & Oils and Fats & $\begin{array}{c}\text { Sugar, } \\
\text { Beverages }\end{array}$ \\
\hline Hypermarket & 0.025 & -0.009 & -0.009 & 0.013 & $0.043 *$ & $0.053^{*}$ & 0.007 & 0.009 & $0.040^{* * * *}$ \\
\hline Supermarket & $\begin{array}{c}(0.031) \\
0.011\end{array}$ & $\begin{array}{c}(0.019) \\
0.003\end{array}$ & $\begin{array}{c}(0.018) \\
-0.031^{* * *}\end{array}$ & $\begin{array}{c}(0.020) \\
0.027\end{array}$ & $\begin{array}{l}(0.023) \\
0.030^{*}\end{array}$ & $\begin{array}{c}(0.029) \\
0.055 * * *\end{array}$ & $\begin{array}{c}(0.006) \\
0.005\end{array}$ & $\begin{array}{c}(0.005) \\
0.005\end{array}$ & $\begin{array}{l}(0.008) \\
0.015 *\end{array}$ \\
\hline & $(0.018)$ & $(0.012)$ & $(0.015)$ & $(0.021)$ & $(0.016)$ & $(0.020)$ & $(0.003)$ & $(0.004)$ & $(0.008)$ \\
\hline Convenience store & $\begin{array}{l}0.058 * * \\
(0.025)\end{array}$ & $\begin{array}{l}-0.007 \\
(0.011)\end{array}$ & $\begin{array}{c}-0.039^{*} \\
(0.023)\end{array}$ & $\begin{array}{c}0.012 \\
(0.019)\end{array}$ & $\begin{array}{c}0.022 \\
(0.015)\end{array}$ & $\begin{array}{c}0.014 \\
(0.050)\end{array}$ & $\begin{array}{c}0.002 \\
(0.005)\end{array}$ & $0.014^{* * *}$ & $\begin{array}{c}0.020 \\
(0.013)\end{array}$ \\
\hline Fast-food restaurant & $\begin{array}{c}-0.100 * * * \\
(0.037)\end{array}$ & & & & $\begin{array}{l}0.110^{*} \\
(0.062)\end{array}$ & $\begin{array}{l}0.132 * * \\
(0.055)\end{array}$ & & & $\begin{array}{l}0.105 * * \\
(0.049)\end{array}$ \\
\hline Grocery store & $\begin{array}{c}0.013 \\
(0.016)\end{array}$ & $\begin{array}{l}-0.003 \\
(0.013)\end{array}$ & $\begin{array}{c}-0.030^{*} \\
(0.017)\end{array}$ & $\begin{array}{c}0.016 \\
(0.023)\end{array}$ & $\begin{array}{c}0.026 \\
(0.016)\end{array}$ & $\begin{array}{c}0.063 * * \\
(0.029)\end{array}$ & $\begin{array}{l}0.008^{* *} \\
(0.004)\end{array}$ & $\begin{array}{c}0.005 \\
(0.004)\end{array}$ & $\begin{array}{c}0.028 * * * \\
(0.007)\end{array}$ \\
\hline Traditional market & $\begin{array}{c}0.011 \\
(0.018)\end{array}$ & $\begin{array}{c}0.016 \\
(0.013)\end{array}$ & $\begin{array}{c}-0.033^{* * *} \\
(0.015)\end{array}$ & $\begin{array}{c}0.058^{* * * *} \\
(0.015)\end{array}$ & $\begin{array}{l}0.015 \\
(0.015)\end{array}$ & $\begin{array}{c}0.023 \\
(0.022)\end{array}$ & $\begin{array}{c}0.004 \\
(0.003)\end{array}$ & $\begin{array}{c}0.011^{* * * *} \\
(0.004)\end{array}$ & $\begin{array}{c}0.024 * * * \\
(0.008)\end{array}$ \\
\hline Roadside market & $\begin{array}{c}0.010 \\
(0.019)\end{array}$ & $\begin{array}{c}0.012 \\
(0.013)\end{array}$ & $\begin{array}{c}-0.038^{* *} \\
(0.018)\end{array}$ & $\begin{array}{c}0.038^{* *} \\
(0.016)\end{array}$ & $\begin{array}{c}0.007 \\
(0.016)\end{array}$ & $\begin{array}{c}0.038^{* * *} \\
(0.015)\end{array}$ & $\begin{array}{c}0.006 \\
(0.004)\end{array}$ & $\begin{array}{c}0.005 \\
(0.004)\end{array}$ & $\begin{array}{c}0.010 \\
(0.007)\end{array}$ \\
\hline Neighborhood kiosk & $\begin{array}{l}0.030 \\
(0.027)\end{array}$ & $\begin{array}{l}-0.013 \\
(0.015)\end{array}$ & $\begin{array}{l}-0.014 \\
(0.017)\end{array}$ & $\begin{array}{l}-0.008 \\
(0.025)\end{array}$ & $\begin{array}{l}-0.010 \\
(0.019)\end{array}$ & $\begin{array}{l}0.057 * * \\
(0.027)\end{array}$ & $\begin{array}{c}0.017 * * * \\
(0.004)\end{array}$ & $\begin{array}{c}0.007 \\
(0.005)\end{array}$ & $\begin{array}{l}0.027^{*} \\
(0.015)\end{array}$ \\
\hline Male & $\begin{array}{c}0.217 \\
(0.478)\end{array}$ & $\begin{array}{c}0.687 * * * * \\
(0.141)\end{array}$ & $\begin{array}{l}-0.020 \\
(0.308)\end{array}$ & $\begin{array}{c}0.765 \\
(0.469)\end{array}$ & $\begin{array}{l}0.471^{*} \\
(0.249)\end{array}$ & $\begin{array}{l}-0.148 \\
(0.531)\end{array}$ & $\begin{array}{c}0.053 \\
(0.085)\end{array}$ & $\begin{array}{c}0.421 * * * \\
(0.092)\end{array}$ & $\begin{array}{c}0.173 \\
(0.193)\end{array}$ \\
\hline Age & $\begin{array}{c}0.025 \\
(0.021)\end{array}$ & $\begin{array}{c}0.007 \\
(0.008)\end{array}$ & $\begin{array}{l}-0.002 \\
(0.011)\end{array}$ & $\begin{array}{l}-0.003 \\
(0.014)\end{array}$ & $\begin{array}{c}-0.0003 \\
(0.007)\end{array}$ & $\begin{array}{c}0.015 \\
(0.018)\end{array}$ & $\begin{array}{c}0.003 \\
(0.004)\end{array}$ & $\begin{array}{c}0.009 * * * \\
(0.003)\end{array}$ & $\begin{array}{c}0.021 * * * \\
(0.006)\end{array}$ \\
\hline Household size & $\begin{array}{c}0.955 * * * \\
(0.123)\end{array}$ & $\begin{array}{c}0.208 * * * \\
(0.073)\end{array}$ & $\begin{array}{l}-0.034 \\
(0.072)\end{array}$ & $\begin{array}{c}0.342 * * * \\
(0.088)\end{array}$ & $\begin{array}{l}0.128^{*} \\
(0.076)\end{array}$ & $\begin{array}{c}0.281 \\
(0.208)\end{array}$ & $\begin{array}{l}-0.004 \\
(0.020)\end{array}$ & $\begin{array}{l}0.056 * * \\
(0.028)\end{array}$ & $\begin{array}{c}0.139 \\
(0.101)\end{array}$ \\
\hline Education & $\begin{array}{c}0.049 \\
(0.049)\end{array}$ & $\begin{array}{c}0.034 \\
(0.032)\end{array}$ & $\begin{array}{l}0.064^{*} \\
(0.036)\end{array}$ & $\begin{array}{l}-0.045 \\
(0.065)\end{array}$ & $\begin{array}{c}0.121 * * * \\
(0.035)\end{array}$ & $\begin{array}{c}0.118 \\
(0.099)\end{array}$ & $\begin{array}{c}0.057 * * * \\
(0.020)\end{array}$ & $\begin{array}{c}-0.022^{*} \\
(0.012)\end{array}$ & $\begin{array}{c}0.086 * * * \\
(0.023)\end{array}$ \\
\hline Income $(\log )$ & $\begin{array}{l}0.374 * \\
(0.193)\end{array}$ & $\begin{array}{l}-0.098 \\
(0.127)\end{array}$ & $\begin{array}{c}0.016 \\
(0.114)\end{array}$ & $\begin{array}{c}0.032 \\
(0.197)\end{array}$ & $\begin{array}{c}0.462 * * * \\
(0.171)\end{array}$ & $\begin{array}{c}0.864 * * * \\
(0.263)\end{array}$ & $\begin{array}{l}0.106 * * \\
(0.043)\end{array}$ & $\begin{array}{l}0.089^{*} \\
(0.047)\end{array}$ & $\begin{array}{c}0.195 \\
(0.148)\end{array}$ \\
\hline Chewa & $\begin{array}{l}0.953^{*} \\
(0.537)\end{array}$ & $\begin{array}{c}0.646^{* * *} \\
(0.301)\end{array}$ & $\begin{array}{c}0.086 \\
(0.389)\end{array}$ & $\begin{array}{l}-0.287 \\
(0.718)\end{array}$ & $\begin{array}{c}0.725^{* *} \\
(0.292)\end{array}$ & $\begin{array}{l}-0.750 \\
(0.728)\end{array}$ & $\begin{array}{c}0.078 \\
(0.109)\end{array}$ & $\begin{array}{c}0.500^{* * * *} \\
(0.103)\end{array}$ & $\begin{array}{c}0.546 \\
(0.332)\end{array}$ \\
\hline Tonga & $\begin{array}{c}0.290 \\
(0.526)\end{array}$ & $\begin{array}{c}0.473^{* * *} \\
(0.204)\end{array}$ & $\begin{array}{l}-0.409 \\
(0.282)\end{array}$ & $\begin{array}{c}1.040 * * \\
(0.426)\end{array}$ & $\begin{array}{c}0.429 \\
(0.310)\end{array}$ & $\begin{array}{c}0.312 \\
(0.505)\end{array}$ & $\begin{array}{c}0.045 \\
(0.149)\end{array}$ & $\begin{array}{c}0.234 * * * \\
(0.073)\end{array}$ & $\begin{array}{l}-0.207 \\
(0.218)\end{array}$ \\
\hline Catholic & $\begin{array}{c}0.100 \\
(0.424)\end{array}$ & $\begin{array}{c}0.265 \\
(0.242)\end{array}$ & $\begin{array}{c}0.239 \\
(0.304)\end{array}$ & $\begin{array}{c}0.764 \\
(0.466)\end{array}$ & $\begin{array}{l}-0.297 \\
(0.340)\end{array}$ & $\begin{array}{l}-0.275 \\
(0.335)\end{array}$ & $\begin{array}{c}0.121 \\
(0.094)\end{array}$ & $\begin{array}{l}-0.003 \\
(0.084)\end{array}$ & $\begin{array}{c}0.039 \\
(0.167)\end{array}$ \\
\hline Seventh Day Adventist & $\begin{array}{c}1.025 * * * \\
(0.384)\end{array}$ & $\begin{array}{c}0.224 \\
(0.265)\end{array}$ & $\begin{array}{c}0.720 * * * \\
(0.219)\end{array}$ & $\begin{array}{c}0.366 \\
(0.574)\end{array}$ & $\begin{array}{l}-0.462 \\
(0.434)\end{array}$ & $\begin{array}{c}0.452 \\
(0.510)\end{array}$ & $\begin{array}{l}-0.078 \\
(0.119)\end{array}$ & $\begin{array}{l}0.129^{*} \\
(0.071)\end{array}$ & $\begin{array}{l}-0.312 \\
(0.211)\end{array}$ \\
\hline Constant & $\begin{array}{l}-0.852 \\
(3.222)\end{array}$ & $\begin{array}{l}-0.848 \\
(1.862)\end{array}$ & $\begin{array}{c}0.741 \\
(1.744)\end{array}$ & $\begin{array}{l}-1.149 \\
(2.243)\end{array}$ & $\begin{array}{c}-5.015^{*} \\
(2.774)\end{array}$ & $\begin{array}{c}-19.214 * * * \\
(5.136)\end{array}$ & $\begin{array}{c}-2.542 * * * \\
(0.711)\end{array}$ & $\begin{array}{c}-1.830 * * * \\
(0.563)\end{array}$ & $\begin{array}{c}-5.442 * * * \\
(1.490)\end{array}$ \\
\hline Pseudo-R-squared & 0.065 & 0.045 & 0.044 & 0.028 & 0.086 & 0.101 & 0.074 & 0.085 & 0.034 \\
\hline Observations & 475 & 475 & 475 & 475 & 475 & 475 & 475 & 475 & 475 \\
\hline
\end{tabular}

Notes: Tobit estimates are shown with robust standard errors clustered at compound level in parentheses. All types of retailers are represented by the household expenditure share for this retailer. * significant at the $10 \%$ level; ** significant at the $5 \%$ level; *** significant at the $1 \%$ level. 
Table A2.10: Associations between the use of different retailers and the consumption of food groups (in value terms)

\begin{tabular}{|c|c|c|c|c|c|c|c|c|}
\hline & \multicolumn{8}{|c|}{ Food expenditure (ZMW/week) } \\
\hline & $\begin{array}{l}\text { Cereals and } \\
\text { Tubers }\end{array}$ & Legumes & Fruits & Vegetables & Meat and Fish & $\begin{array}{c}\text { Dairy and } \\
\text { Eggs }\end{array}$ & Oils and Fats & Sugar, Beverages \\
\hline \multicolumn{9}{|c|}{ Panel A:Only supermarkets considered } \\
\hline \multirow[t]{2}{*}{ Supermarket } & 0.095 & -0.015 & $-0.315^{* *}$ & $-0.258 * * *$ & $0.445^{* * *}$ & -0.059 & -0.017 & -0.086 \\
\hline & $(0.069)$ & $(0.134)$ & $(0.140)$ & $(0.081)$ & $(0.167)$ & $(0.053)$ & $(0.032)$ & $(0.054)$ \\
\hline Other covariates & Yes & Yes & Yes & Yes & Yes & Yes & Yes & Yes \\
\hline \multicolumn{9}{|c|}{ Panel B: Multiple retailers considered } \\
\hline \multirow[t]{2}{*}{ Hypermarket } & $0.863^{* *}$ & 0.025 & 0.248 & -0.300 & $1.115^{*}$ & $0.895 * *$ & $0.237 * * *$ & 0.109 \\
\hline & $(0.358)$ & $(0.422)$ & $(0.813)$ & $(0.267)$ & $(0.664)$ & $(0.366)$ & $(0.066)$ & $(0.133)$ \\
\hline \multirow[t]{2}{*}{ Supermarket } & 0.233 & 0.197 & $-0.866^{* * *}$ & -0.066 & $1.093^{* * *}$ & $0.284^{*}$ & $0.159^{* *}$ & -0.011 \\
\hline & $(0.177)$ & $(0.258)$ & $(0.330)$ & $(0.244)$ & $(0.381)$ & $(0.152)$ & $(0.069)$ & $(0.149)$ \\
\hline \multirow[t]{2}{*}{ Convenience store } & $0.952 * *$ & $0.521^{*}$ & $-1.084 *$ & 0.446 & 0.436 & $0.384 * *$ & $0.255^{* * * *}$ & 0.024 \\
\hline & $(0.409)$ & $(0.284)$ & $(0.564)$ & $(0.410)$ & $(0.356)$ & $(0.173)$ & $(0.077)$ & $(0.220)$ \\
\hline \multirow[t]{2}{*}{ Fast-food restaurant } & 0.866 & & & & $4.527 * *$ & 1.518 & & 0.355 \\
\hline & (1.317) & & & & $(1.789)$ & $(1.231)$ & & $(0.369)$ \\
\hline \multirow[t]{2}{*}{ Grocery store } & 0.189 & -0.035 & $-0.740^{* *}$ & -0.021 & $0.879 * *$ & $0.348^{*}$ & $0.140^{* *}$ & 0.074 \\
\hline & $(0.180)$ & $(0.242)$ & $(0.342)$ & $(0.268)$ & $(0.400)$ & $(0.209)$ & $(0.065)$ & $(0.123)$ \\
\hline \multirow[t]{2}{*}{ Traditional market } & 0.008 & $0.546^{* *}$ & $-0.711 * *$ & $0.418^{* *}$ & $0.722 *$ & 0.203 & $0.220^{* * * *}$ & 0.054 \\
\hline & $(0.141)$ & $(0.254)$ & $(0.294)$ & $(0.196)$ & $(0.379)$ & $(0.150)$ & $(0.062)$ & $(0.130)$ \\
\hline \multirow[t]{2}{*}{ Roadside market } & -0.143 & $0.417 *$ & $-0.878 * * *$ & $0.593 * * *$ & 0.508 & $0.329 * *$ & $0.110^{*}$ & -0.111 \\
\hline & $(0.168)$ & $(0.225)$ & $(0.301)$ & $(0.173)$ & $(0.383)$ & $(0.164)$ & $(0.063)$ & $(0.129)$ \\
\hline \multirow[t]{2}{*}{ Neighborhood kiosk } & 0.229 & -0.239 & -0.108 & 0.017 & 0.146 & $0.670^{* * * *}$ & $0.219^{* *}$ & 0.282 \\
\hline & $(0.221)$ & $(0.279)$ & $(0.445)$ & $(0.310)$ & $(0.477)$ & $(0.177)$ & $(0.091)$ & $(0.262)$ \\
\hline Other covariates & Yes & Yes & Yes & Yes & Yes & Yes & Yes & Yes \\
\hline Observations & 475 & 475 & 475 & 475 & 475 & 475 & 475 & 475 \\
\hline
\end{tabular}

Notes: Tobit estimates are shown with robust standard errors clustered at compound level in parentheses. All types of retailers are represented by the household expenditure share for this retailer. Socioeconomic control variables are included in all models, but are not shown here for brevity. * significant at the $10 \%$ level; ** significant at the $5 \%$ level; *** significant at the $1 \%$ levels. 


\title{
3 Effects of modern food retailers on adult and child diets and nutrition ${ }^{2}$
}

\begin{abstract}
In many developing countries, food environments are changing rapidly, with modern retailers such as supermarkets and convenience stores - increasingly replacing traditional markets and shops. Previous studies suggested that the rise of modern retailers contributes to overweight and obesity. Effects on dietary quality were not analyzed before due to the unavailability of individuallevel dietary data. Here, we analyze effects of modern retailers on dietary quality and nutrition in Lusaka, Zambia. We collected data from randomly selected households that use modern retailers at different intensities. Individual-level anthropometric and food-intake data from 930 adults and 499 children are analyzed with control function regression models to estimate effects of modern retailers on body weight, height, dietary diversity, and nutrient intakes. Use of modern retailers is positively associated with overweight in adults, but not in children. For children, we find a positive effect on body height. Use of modern retailers increases dietary diversity, as well as calorie, protein, and micronutrient intakes among both adults and children. Effects on protein and micronutrient intakes are channeled primarily through higher consumption of meat and dairy products. The findings underline that the growth of modern retailers influences people's diets and nutrition; the effects can be both positive and negative. The positive effects on child nutrition imply that further modernization of food environments should be promoted. But the increasing effect on adult overweight is undesirable and calls for certain policy regulations. While the results cannot be generalized, effects may be similar also in other parts of Africa.
\end{abstract}

Keywords: Child undernutrition; overweight; obesity; food environments; supermarkets; Africa.

\footnotetext{
${ }^{2}$ This paper has been co-authored with Olivier Ecker (O.E.) and Matin Qaim (M.Q.). The research idea was jointly conceptualized and designed by I, Makaiko Gonapanyanja Khonje (M.G.K.) and M.Q.. O.E. contributed to the design. M.G.K. collected the data and did the data analysis in consultation with O.E., and M.Q.. M.G.K., and M.Q. wrote the manuscript. All authors read and approved the final version of the manuscript.
} 


\subsection{Introduction}

Malnutrition is a global problem with serious negative health implications (FAO et al., 2019; Swinburn et al., 2019). Especially in developing countries, different types of malnutrition typically coexist within the same communities, households, and even individuals (Development Initiatives, 2018; Fongar et al., 2019; Hawkes et al., 2020; Popkin et al., 2020). While undernutrition and micronutrient deficiencies remain widespread, overweight and obesity are rapidly on the rise (Development Initiatives, 2018; FAO et al., 2019; Fongar et al., 2019; Swinburn et al., 2019; Hawkes et al., 2020; Popkin et al., 2020). Food environments, defined as the physical, economic, and sociocultural context in which consumers acquire their food, can influence dietary choices and nutrition (HLPE, 2017; Popkin, 2017; Hawkes et al., 2020). And food environments are changing rapidly.

In many developing countries, modern retailers - including supermarkets, hypermarkets, convenience stores, and fast-food restaurants - are gaining in importance at the expense of traditional food markets and shops (Popkin, 2014; Reardon and Timmer, 2014; Qaim, 2017). Due to higher efficiency and economies-of-scale, modern retailers may improve consumers' access to affordable foods (Hawkes, 2008; Tessier et al., 2008; Popkin, 2014; Qaim, 2017). On the other hand, modern retailers tend to sell more processed foods than traditional markets (Popkin, 2017; Khonje and Qaim, 2019; Swinburn et al., 2019). Highly processed foods are often rich in fat, sugar, and salt, but poor in micronutrients. Hence, the growth of modern retailers in developing countries may increase calorie consumption without necessarily improving dietary quality. Possibly, modern retailers may even worsen dietary quality through promoting the consumption of unhealthy snacks, beverages, and convenience foods (Popkin, 2017; Popkin and Reardon, 2018; Hawkes et al., 2020).

A few recent studies analyzed the effects of modern retailers on diets and nutrition in developing countries. Most of these studies focused on the role of supermarkets. Studies in Guatemala, Indonesia, Kenya, and Thailand showed that purchasing food in supermarkets leads to more consumption of processed and highly-processed foods (Asfaw, 2008; Kelly et al., 2014; Rischke et al., 2015; Umberger et al., 2015). In Guatemala and Kenya, supermarket food purchases were also shown to contribute to rising body mass index (BMI) and increased prevalence rates of overweight, obesity, and related chronic diseases among adults (Asfaw, 2008; Kimenju et al., 2015; Demmler et al., 2017; Demmler et al., 2018). 
Effects of modern retailers on child nutrition were rarely analyzed. One recent study with data from Kenya showed that supermarkets do not contribute to childhood obesity but have a positive effect on child linear growth and height (Debela et al., 2020). Positive effects on child height are surprising, as linear growth is known to be closely related to dietary quality and balanced nutrient intakes (Ruel and Alderman, 2013; Development Initiatives, 2018). Do supermarkets and other modern retailers really contribute to improved nutrient intakes? This is an important question, which none of the previous studies was able to answer. Previous studies collected food consumption data at the household level, suggesting that purchasing food in supermarkets can lead to higher dietary diversity in some situations (Rischke et al., 2015; Rupa et al., 2019; Debela et al., 2020). But household-level data neglect intra-household food distribution and are therefore not necessarily good proxies of individual-level dietary quality and micronutrient intakes.

We provide the first study that analyzes effects of modern retailers on diets and nutrition with individual-level food-intake and anthropometric data from adults and children in a developing country. We use cross-section observational data collected in urban Zambia. Like many other countries in sub-Saharan Africa, Zambia is characterized by a high prevalence of different forms of malnutrition and a rapid modernization of food environments (Harris et al., 2019; Khonje and Qaim, 2019).

\subsection{Methods}

\subsubsection{Survey of households and individuals}

Data for this study were collected through a survey of households and individuals in Lusaka, the Capital City of Zambia. The survey was implemented between April and July 2018. As many other large cities in sub-Saharan Africa, Lusaka has recently experienced a rapid growth of modern retailers. The number of large shopping malls in Lusaka increased from one in 1995 to 25 in 2018 (Table 1.1). Shopping malls often include hypermarkets, supermarkets, and fast-food restaurants. In addition, the number of supermarkets and convenience stores outside of large shopping malls has also grown substantially.

We surveyed a total of 475 households from several compounds/sections in Lusaka City using a two-stage sampling procedure. At the first stage, we purposively selected 14 compounds/sections as primary sampling units based on population distributions and information from the Lusaka City 
Council on mean income levels in the different compounds or sections. To ensure that the sample is representative and covers a wide variation of socioeconomic situations, we selected four compounds/sections (Avondole, Chalala, Kabulonga, and Woodlands) with high mean income levels, four compounds/sections (Chelston, Chilenje, Kabwata, and PHI) with middle mean income levels, and six compounds/sections (Chawama, Chazanga, Gardens, Kalingalinga, Kaunda Square, and Ng'ombe) with low mean income levels (Figure 1.1).

At the second stage, depending on the size of the compound/section, we randomly sampled about 35 households from each compound/section for study participation. In each sampled household, we interviewed the household head or the adult responsible for food purchase decisions and food preparation. We recruited local enumerators to conduct face-to-face interviews in local languages. The enumerators were trained and supervised by the researchers. The structured questionnaire covered sections on the household demographic structure, economic activities, income, and consumption expenditures. Data on food consumption at the household level were collected through a seven-day recall using a list of 140 different food items and capturing quantities, prices, and sources of each item.

Food-intake and nutrition data were captured at the individual level for up to four randomly selected members of each household: two adults ( $\geq 18$ years) and two children/adolescents (6 months - 18 years). In this article, we use the term "children" for all individuals $<18$ years. Individual-level food-intake data were collected through 24-hour dietary recalls; for small children the recall questions were answered by the caregiver. Weight and height of all participating individuals were also measured. We have complete individual-level data for 930 adults (623

female and 307 male adults) and 499 children (Table A3.1 in the Appendix). All participating adults provided written informed consent for themselves and for their children. The study was reviewed and approved by the Ethics Committee of the University of Goettingen.

\subsubsection{Measuring nutrition and dietary quality}

Nutritional status of adults and children is evaluated with body height and weight measures. For adults, we calculate the body mass index (BMI), whereby $\mathrm{BMI}>25.0 \mathrm{~kg} / \mathrm{m}^{2}$ is defined as overweight or obese. For children, we calculate BMI-for-age Z-scores (BAZ) and height-for-age Z- 
scores (HAZ) (WHO, 2006). Child overweight/obesity is defined as BAZ>2 standard deviations (SD), child stunting as $\mathrm{HAZ}<-2 \mathrm{SD}$.

Dietary quality is evaluated with individual-level food-intake data. Simple dietary quality indicators are the food variety score (FVS), dietary diversity score (DDS), and healthy eating index (HEI) (Steyn et al., 2006; Demmler et al., 2018). FVS is as a simple count of the different food items eaten by the individual during the 24-hour recall period. DDS is a count of the number of different food groups eaten, considering a total of nine healthy food groups (Table A3.2 in the Appendix). FVS and DDS do not take into account the quantities of foods consumed. In contrast, HEI takes into account the quantities of 13 food components consumed, using a scoring method for the calculation (Table A3.2). HEI ranges from zero to 100, with higher values indicating better dietary quality (USDA, 2019). To our knowledge, HEI has been used as an indicator of dietary quality in the United States and other developed countries, but not yet in developing countries.

Other indicators of nutritional quality that we use are individual-level intakes of calories, protein, and several micronutrients, namely iron, zinc, and vitamin A. While the human body needs a large number of micronutrients for healthy nutrition, deficiencies in these three micronutrients are particularly common in developing countries and responsible for large health problems among children and adults (Development Initiatives, 2018). Calorie and nutrient intakes are calculated based on the quantities of the different food items consumed and local food composition tables (Nyirenda et al., 2009; FAO and Government of Kenya, 2018).

We also calculate micronutrient adequacy ratios for iron, zinc, and vitamin A, taking into account estimated average requirements for individual age and sex groups (Table A3.3 in the Appendix). Mean adequacy ratios are calculated by summing the adequacy ratios (truncated at 100\%) for the three micronutrients and dividing by three (Steyn et al., 2006)

\subsubsection{Statistical analysis}

We analyze the effect of purchasing food in modern retailers on individual-level diets and nutrition with regression models of the following type:

$$
N_{i}=\alpha+\beta M R_{h i}+\gamma X_{i}+\delta X_{h i}+\varepsilon_{i}
$$


where $N_{i}$ is the nutrition status or dietary quality indicator of individual $i$, and $M R_{h i}$ is the main explanatory variable of interest measuring the use of modern retailers for food purchases in household $h$ that individual $i$ belongs to. Modern retailers include hypermarkets, supermarkets, convenience stores, and fast-food restaurants. We define $M R_{h i}$ as the share of total household food expenditures made in modern retailers expressed in percent and referring to the seven-day food consumption recall at the household level. In some of the models, we alternatively define $M R_{h i}$ as a dummy variable that takes a value of one if the household purchased any of the food items consumed in a modern retailer, and zero if all of the foods were obtained from traditional sources (traditional markets, groceries, mom-and-pop-shops, kiosks, own production etc.). The estimation coefficient $\beta$ indicates whether food purchases in modern retailers influence individual diets and nutrition.

Diets and nutrition can also be influenced by several other factors that we control for in the regression models. We control for individual characteristics $\left(X_{i}\right)$, such as age, education, ethnicity, and religion, and also for relevant household characteristics $\left(X_{h i}\right)$, such as household size and income (Asfaw, 2008; Demmler et al., 2018; Debela et al., 2020). $\varepsilon_{i}$ is a random error term. We estimate all models separately for adults and children. For outcome variables that are measured with count data (FVS, DDS, HEI) we use Poisson models for estimation. Overweight/obese is a dummy variable, for which we use a probit specification. Several other outcomes are censored variables (calorie and nutrient intakes) for which we use Tobit specifications. For dependent variables that are continuous and normally distributed (BMI, HAZ), we use linear regression models. We use and report bootstrapped standard errors clustered at the compound level. All data analyses are performed with the software package Stata/SE 15.1.

\subsubsection{Control function approach}

In the regression models in equation (3.1), the main explanatory variable of interest ("treatment" variable) is food purchases in modern retailers $\left(M R_{h i}\right)$, which is likely endogenous. What type of retailers to use for food purchases is a household-level decision that may depend on various factors not all of which we can observe and control for. If unobserved factors are jointly correlated with $M R_{h i}$ and with the dietary and nutrition outcome variables, the coefficient estimate $\beta$ may be biased. 
We use a control function (CF) approach with instrumental variables (IV) to test and correct for such endogeneity bias (Wooldridge, 2010; 2015). In comparison to standard IV regressions, the CF estimator is more efficient and can also be used to control for unobserved heterogeneity in nonlinear models, such as probit and Poisson models (Wooldridge, 2015). The CF is estimated as a two-stage model, whereby the first-stage regression is represented as:

$$
M R_{h i}=\theta_{0}+\theta_{1} X_{i}+\theta_{2} X_{h i}+\theta_{3} Z_{h i}+\mu_{i}
$$

where $Z_{h i}$ is a vector of instrumental variables, and $\mu_{i}$ is a random error term. The other variables are defined as above. The second-stage model is the regression shown in equation (3.1) with the individual diet and nutrition outcomes as dependent variable but including the residuals from the first-stage model as an additional regressor. If the residual term is statistically insignificant in the second-stage regression, the null hypothesis of no endogeneity bias cannot be rejected, so that the $\mathrm{CF}$ approach is not required; in that case, regular one-stage models lead to unbiased and more efficient estimates. However, if the residual term turns out statistically significant, the CF approach is preferred and controls for endogeneity bias.

We use three instrumental variables for the CF models. First, distance from each household to the closest shopping mall, which is calculated using global positioning system (GPS) data collected during the survey. GPS-based data to measure distance to modern retailers were also used as instruments in several other studies (Kimenju et al., 2015; Rischke et al., 2015; Umberger et al., 2015; Debela et al., 2020). Second, whether or not the household feels that modern retailers sell food of higher quality than traditional retailers, which was also used by Umberger et al. (2015). Third, the number of visits to a shopping mall of the household's closest neighbor in the sample. Beyond distance and accessibility, the neighbor's behavior may capture influence through local social networks (Rupa et al., 2019). Tests for instrument validity are discussed below.

All three instruments are significantly correlated with the household's own use of modern retailers, $M R_{h i}$ (Table A3.4 in the Appendix). As expected, distance to the closest shopping mall is negatively correlated, while the other two instruments are positively correlated with the food expenditure share spent in modern retailers. Moreover, the Wald test for the joint significance of the three instruments is statistically significant at the $1 \%$ level for both adults and children (Table A3.4). This underlines that the instruments are relevant. A second important criterion for validity is that the instruments do not affect the dietary and nutrition outcome variables directly, other than 
through own use of modern retailers. We perform a simple falsification test following Di Falco et al. (2011). Results show that the three instruments are jointly insignificant in all models with dietary and nutrition outcomes as dependent variables (Table A3.5 in the Appendix). Hence, we conclude that the instruments are valid.

\subsection{Results}

\subsubsection{Descriptive comparisons}

Three-quarters of all households in the sample used modern retailers during the seven days prior to the survey, at least for some of their food purchases. The rest obtained all of the foods consumed from traditional sources. Users of modern retailers spend $59 \%$ of their total food expenditures in modern retailers on average. Table 3.1 shows individual-level food intakes of adults and children. Differentiation is made between individuals from households with and without the use of modern retailers. Table 3.1 show that users of modern retailers consume lower quantities of vegetables and pulses and higher quantities of meat, dairy, sugar, and beverages than non-users. This comparison points at notable dietary differences between the two groups.

Table 3.1: Per capita food intake of adults and children using and not using modern retailers

\begin{tabular}{|c|c|c|c|c|}
\hline \multirow{3}{*}{ Food intake (g/day) } & \multicolumn{2}{|c|}{ Adults $(\geq 18$ years) } & \multicolumn{2}{|c|}{ Children $(<18$ years $)$} \\
\hline & \multicolumn{2}{|c|}{ Modern retailers } & \multicolumn{2}{|c|}{ Modern retailers } \\
\hline & $\begin{array}{c}\text { Users } \\
(\mathrm{N}=713)\end{array}$ & $\begin{array}{c}\text { Non-users } \\
(\mathrm{N}=217)\end{array}$ & $\begin{array}{c}\text { Users } \\
(\mathrm{N}=358)\end{array}$ & $\begin{array}{c}\text { Non-users } \\
(\mathrm{N}=141)\end{array}$ \\
\hline Cereals and tubers & $\begin{array}{c}569.18 \\
(288.83)\end{array}$ & $\begin{array}{c}576.57 \\
(298.11)\end{array}$ & $\begin{array}{c}427.77 \\
(232.81)\end{array}$ & $\begin{array}{c}396.39 \\
(225.30)\end{array}$ \\
\hline Pulses & $\begin{array}{c}12.36 * * * \\
(39.41)\end{array}$ & $\begin{array}{c}24.07 \\
(89.14)\end{array}$ & $\begin{array}{l}9.18 * * \\
(25.95)\end{array}$ & $\begin{array}{c}22.77 \\
(100.90)\end{array}$ \\
\hline Vegetables & $\begin{array}{c}47.33 * * * \\
(71.80)\end{array}$ & $\begin{array}{c}78.05 \\
(123.14)\end{array}$ & $\begin{array}{c}31.82 * * * \\
(63.30)\end{array}$ & $\begin{array}{c}58.28 \\
(89.26)\end{array}$ \\
\hline Fruits & $\begin{array}{c}3.30 \\
(18.94)\end{array}$ & $\begin{array}{c}3.04 \\
(21.75)\end{array}$ & $\begin{array}{c}1.19 * * * \\
(5.47)\end{array}$ & $\begin{array}{c}4.09 \\
(17.41)\end{array}$ \\
\hline Meat & $\begin{array}{c}36.66 * * * \\
(43.80)\end{array}$ & $\begin{array}{c}22.64 \\
(47.43)\end{array}$ & $\begin{array}{c}26.82 * * * \\
(31.40)\end{array}$ & $\begin{array}{c}14.95 \\
(46.14)\end{array}$ \\
\hline Dairy products & $\begin{array}{c}19.76^{* *} \\
(76.96)\end{array}$ & $\begin{array}{c}7.85 \\
(47.41)\end{array}$ & $\begin{array}{l}22.22 * \\
(59.43)\end{array}$ & $\begin{array}{c}12.41 \\
(58.94)\end{array}$ \\
\hline Eggs & $\begin{array}{c}7.69 \\
(24.07)\end{array}$ & $\begin{array}{c}10.63 \\
(46.93)\end{array}$ & $\begin{array}{c}5.59 * \\
(19.85)\end{array}$ & $\begin{array}{c}10.80 \\
(42.16)\end{array}$ \\
\hline Fish & $\begin{array}{c}19.33 \\
(55.96)\end{array}$ & $\begin{array}{c}23.29 \\
(63.53)\end{array}$ & $\begin{array}{c}11.79 \\
(38.55)\end{array}$ & $\begin{array}{c}14.40 \\
(47.60)\end{array}$ \\
\hline Sugar, beverages & $\begin{array}{c}171.80 * * * \\
(196.37)\end{array}$ & $\begin{array}{c}124.83 \\
(173.95)\end{array}$ & $\begin{array}{c}140.79 * * \\
(171.75)\end{array}$ & $\begin{array}{c}101.25 \\
(130.71)\end{array}$ \\
\hline Oils and fats & $\begin{array}{c}0.65 \\
(2.28) \\
\end{array}$ & $\begin{array}{c}0.59 \\
(1.14) \\
\end{array}$ & $\begin{array}{c}0.56 \\
(2.95) \\
\end{array}$ & $\begin{array}{c}0.59 \\
(1.08) \\
\end{array}$ \\
\hline
\end{tabular}

Mean values are shown with standard deviations in parentheses. t-tests were carried out to test for mean differences between users and non-users of modern retailers. ${ }^{*} \mathrm{p}<0.10,{ }^{* *} \mathrm{p}<0.05,{ }^{* * *} \mathrm{p}<0.01 . \mathrm{N}$, number of observations. 
Table 3.2 shows individual diet and nutrition outcomes with and without modern retailer use. Overweight/obesity is fairly widespread, affecting $40-50 \%$ of the adults with no significant differences between the two groups. For children, overweight/obesity rates are much lower at 56\%. Children are more affected by undernutrition; the prevalence of child stunting is lower among modern retail users than among non-users, even though the difference is not statistically significant. For the dietary indicators, several significant differences are observed. Adults and

Table 3.2: Nutrition and dietary indicators for adults and children using and not using modern retailers

\begin{tabular}{|c|c|c|c|c|c|}
\hline \multirow[t]{2}{*}{ Variables } & \multirow[t]{2}{*}{ Units } & \multicolumn{2}{|c|}{$\begin{array}{c}\text { Adults }(\geq 18 \text { years) } \\
\text { Modern retailers }\end{array}$} & \multicolumn{2}{|c|}{$\begin{array}{c}\text { Children }(<18 \text { years }) \\
\text { Modern retailers } \\
\end{array}$} \\
\hline & & $\begin{array}{c}\text { Users } \\
(\mathrm{N}=713)\end{array}$ & $\begin{array}{l}\text { Non-users } \\
(\mathrm{N}=217)\end{array}$ & $\begin{array}{c}\text { Users } \\
(\mathrm{N}=358)\end{array}$ & $\begin{array}{c}\text { Non-users } \\
(\mathrm{N}=141)\end{array}$ \\
\hline \multirow[t]{2}{*}{ Body mass index (BMI) } & $\begin{array}{l}\mathrm{kg} / \mathrm{m}^{2} \text { or BMI-for-age } \mathrm{Z} \\
\text { score }\end{array}$ & 25.86 & 25.51 & 0.05 & -0.18 \\
\hline & & $(4.88)$ & $(5.65)$ & $(1.45)$ & $(1.72)$ \\
\hline \multirow[t]{2}{*}{ Overweight or obese } & $\begin{array}{l}1 \text { if } \mathrm{BMI} \geq 25 \text { or } \mathrm{BAZ}>2 \\
\mathrm{SD}\end{array}$ & 0.50 & 0.44 & 0.05 & 0.06 \\
\hline & & $(0.50)$ & $(0.50)$ & $(0.22)$ & $(0.24)$ \\
\hline \multirow[t]{2}{*}{$\begin{array}{l}\text { Height-for-age Z score } \\
\text { (HAZ) }\end{array}$} & Z score & NA & NA & -0.51 & -0.72 \\
\hline & & & & $(1.51)$ & $(1.59)$ \\
\hline Stunting & 1 if $\mathrm{HAZ}<-2 \mathrm{SD}$ & NA & NA & $\begin{array}{c}0.15 \\
(0.36)\end{array}$ & $\begin{array}{c}0.21 \\
(0.41)\end{array}$ \\
\hline Food variety score (FVS) & Score; range $(0-18)$ & $\begin{array}{c}6.64 * * \\
(1.85)\end{array}$ & $\begin{array}{l}6.26 \\
(2.11)\end{array}$ & $\begin{array}{l}6.69 * * \\
(1.94)\end{array}$ & $\begin{array}{c}6.28 \\
(1.49)\end{array}$ \\
\hline $\begin{array}{l}\text { Dietary diversity score } \\
\text { (DDS) }\end{array}$ & Score; range (0-9) & $\begin{array}{l}3.23 \\
(1.02)\end{array}$ & $\begin{array}{r}3.12 \\
(1.00)\end{array}$ & 3.02 & $\begin{array}{l}3.08 \\
(1.00)\end{array}$ \\
\hline $\begin{array}{l}\text { Healthy eating index } \\
\text { (HEI) }\end{array}$ & Score; range $(0-100)$ & $\begin{array}{c}32.58 * * * \\
(10.12)\end{array}$ & $\begin{array}{c}29.77 \\
(10.94)\end{array}$ & $\begin{array}{c}31.59 * * * \\
(10.88)\end{array}$ & $\begin{array}{c}28.41 \\
(10.73)\end{array}$ \\
\hline Calorie intake & kcal/day & $\begin{array}{l}2653.11 * * \\
(1161.83)\end{array}$ & $\begin{array}{l}2457.08 \\
(985.42)\end{array}$ & $\begin{array}{l}2006.76 \\
(936.00)\end{array}$ & $\begin{array}{l}1964.00 \\
(969.40)\end{array}$ \\
\hline Protein intake & g/day & $\begin{array}{c}81.28 \\
(35.49)\end{array}$ & $\begin{array}{c}80.96 \\
(39.30)\end{array}$ & $\begin{array}{c}60.44 \\
(33.10)\end{array}$ & $\begin{array}{c}60.62 \\
(34.37)\end{array}$ \\
\hline Iron intake & mg/day & $\begin{array}{c}23.88 \\
(11.71)\end{array}$ & $\begin{array}{c}24.61 \\
(12.84)\end{array}$ & $\begin{array}{l}17.05 \\
(9.50)\end{array}$ & $\begin{array}{c}18.41 \\
(12.19)\end{array}$ \\
\hline Zinc intake & mg/day & $\begin{array}{c}7.59 \\
(5.45)\end{array}$ & $\begin{array}{l}7.64 \\
(6.19)\end{array}$ & $\begin{array}{c}5.36 \\
(3.10)\end{array}$ & $\begin{array}{c}5.47 \\
(5.44)\end{array}$ \\
\hline Vitamin A intake & $\mu \mathrm{g}$ retinol/day & $\begin{array}{c}525.83 * * * \\
(499.93)\end{array}$ & $\begin{array}{c}409.33 \\
(454.70)\end{array}$ & $\begin{array}{l}473.48 * * \\
(487.48)\end{array}$ & $\begin{array}{c}380.22 \\
(428.93)\end{array}$ \\
\hline
\end{tabular}

Mean values are shown with standard deviations in parentheses. t-tests were carried out to test for mean differences between users and non-users of modern retailers. ${ }^{* *} \mathrm{p}<0.05,{ }^{* * *} \mathrm{p}<0.01 . \mathrm{N}$, number of observations; NA, not applicable. SD, standard deviation. Additional variables are shown in Table A3.6 in the Appendix.

children in households using modern retailers have higher food variety scores (FVS), a higher healthy eating index (HEI), and higher vitamin A intakes than their counterparts in households that 
obtain all of their foods from traditional sources. Nevertheless, mean micronutrient adequacy ratios are below 100\% for all subsamples (Table A3.6 in the Appendix).

The differences in dietary and nutrition outcomes between users and non-users of modern retailers observed in Tables 3.1 and 3.2 cannot be interpreted as effects of modern retailers, because the groups also differ in terms of various other characteristics. For instance, users of modern retailers have significantly higher incomes and education levels than non-users (Table A3.6). As explained above, we use regression models with a control function approach to control for such heterogeneity and be able to make causal inference.

\subsubsection{Effects of modern retailers on nutrition status}

Table 3.3 shows estimated net effects of using modern retailers on nutrition status. We start by interpreting the results for adults. After controlling for household income and other relevant factors, a one percentage point increase in the food expenditure share spent in modern retailers increases adult BMI by $0.012 \mathrm{~kg} / \mathrm{m}^{2}$. Equivalently, a 10 percentage point increase in the modern

Table 3.3: Effects of using modern retailers on nutrition status

\begin{tabular}{|c|c|c|c|c|c|}
\hline & \multicolumn{2}{|c|}{ Adults ( $\geq 18$ years) } & \multicolumn{3}{|c|}{ Children $(<18$ years) } \\
\hline & $\begin{array}{c}\text { BMI } \\
\left(\mathrm{kg} / \mathrm{m}^{2}\right)\end{array}$ & $\begin{array}{c}\text { Overweight/Obese } \\
(1,0)\end{array}$ & $\begin{array}{c}\mathbf{B A Z} \\
(\mathrm{Z} \text {-score })\end{array}$ & $\begin{array}{c}\text { Overweight/Obese } \\
(1,0)\end{array}$ & $\begin{array}{c}\text { HAZ } \\
\text { (Z-score) }\end{array}$ \\
\hline & $(1)$ & $(2)$ & (3) & (4) & $(5)$ \\
\hline Modern retail use & $0.012 * *$ & $0.004 * * *$ & -0.011 & $-0.016^{* *}$ & $0.026^{* * *}$ \\
\hline (expenditure share, \%) & $(0.005)$ & $(0.002)$ & $(0.008)$ & $(0.007)$ & $(0.008)$ \\
\hline Control variables & Yes & Yes & Yes & Yes & Yes \\
\hline Joint F-statistic/Wald $\chi^{2}$ & $761 * * *$ & $862 * * *$ & $66 * * *$ & $35 * * *$ & $117 * * *$ \\
\hline $\mathrm{N}$ & 863 & 863 & 458 & 458 & 472 \\
\hline
\end{tabular}

Marginal effects are shown with robust, bootstrapped standard errors clustered at compound level in parentheses. Full model results with all control variables are shown in Table A3.7 in the Appendix. For the adult sample, the null hypothesis of modern retailer use being exogenous could not be rejected, so that standard ordinary least squares and probit estimates are shown. For the child sample, the null hypothesis of exogeneity was rejected, so that control function estimates are shown. ${ }^{* *} \mathrm{p}<0.05,{ }^{* * *} \mathrm{p}<0.01$. BAZ, BMI-for-age Z-score; BMI, body mass index; HAZ, height-for-age Zscore; N, number of observations.

retailer share is associated with a 0.12 increase in adult BMI (the mean expenditure share among modern retail users is $59 \%$, implying a total effect on BMI of $0.7 \mathrm{~kg} / \mathrm{m}^{2}$ ). As mentioned, a considerable proportion of the adults are already overweight or obese. The risk of adult overweight/obesity further rises through the use of modern retailers: a 10 percentage point increase 
in the modern retailer expenditure share raises the likelihood of overweight/obesity by 4 percentage points (Table 3.3).

For children, the results are different. Using modern retailers has no significant effect on child BMI-for-age Z-scores (column 3 of Table 3.3), and the effect on child overweight/obesity is even negative (column 4). At the same time, we observe a statistically significant positive effect on child height (column 5). A 10 percentage point increase in the modern retail expenditure share raises child-for-age Z-scores (HAZ) by 0.26 . This points at clearly positive effects of modern retailers on child nutrition

\subsubsection{Effects of modern retailers on dietary quality}

Figure 3.1 shows effects of modern retailers on adult and child dietary diversity. After controlling for income and other relevant factors, use of modern retailers increases adult FVS and dietary diversity score (DDS) by 10-12\%. For children, the point estimates for FVS and DDS are positive

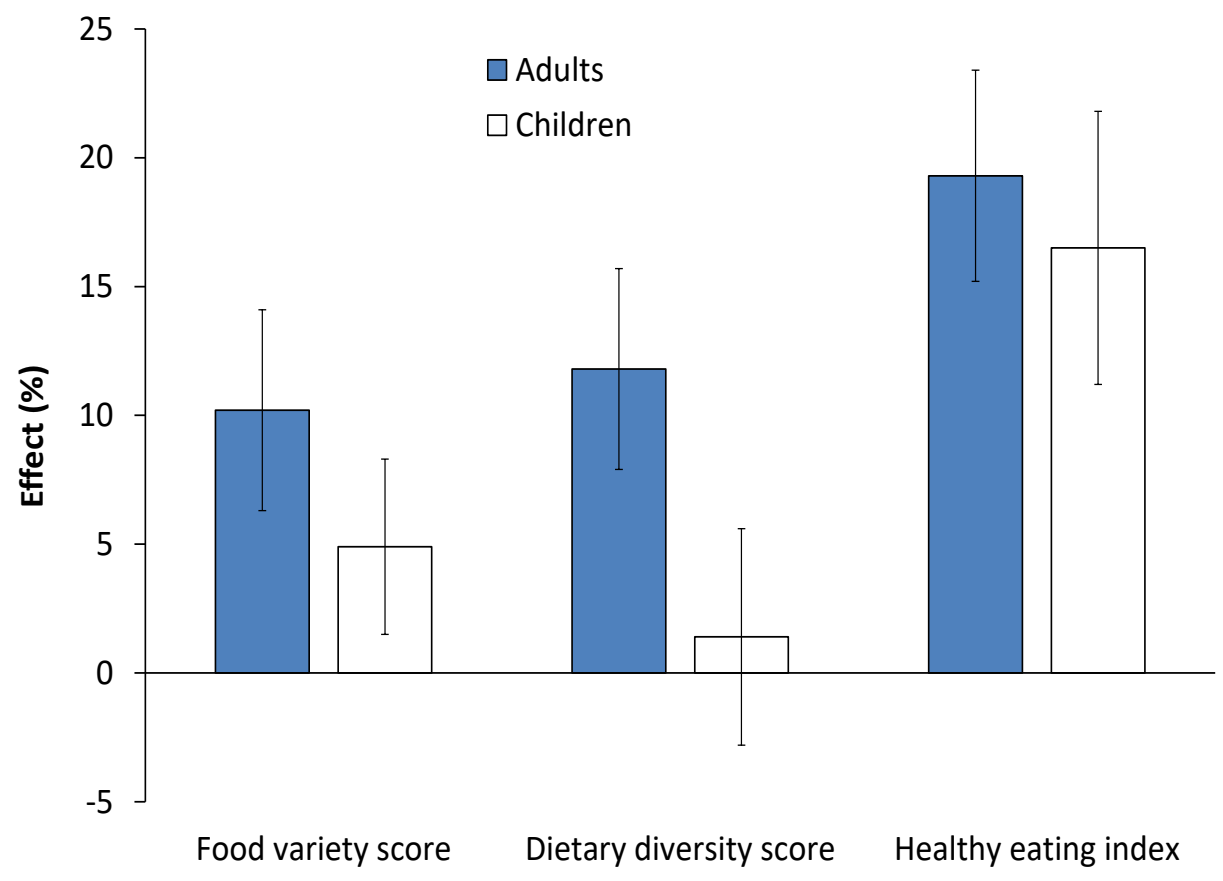

\section{Figure 3.1: Effects of using modern retailers on dietary diversity}

Percentage effects are shown with standard error bars. Use of modern retailers expressed as a dummy variable that takes a value of one if any of the food consumed was purchased in a modern retailer and zero if all of the foods consumed were obtained from traditional sources. Effects were estimated with control function models, controlling for income, education, age, and other relevant factors. Models for adults were estimated with 930 individual observations. Models for children were estimated with 499 individual observations. Full model results are shown in Tables A3.8 and A3.9 in the Appendix. 
but not statistically significant. However, FVS and DDS only count the number of food items and food groups consumed without considering quantities. When considering intake quantities of different food components through the HEI, the effects are larger and statistically significant. Use of modern retailers increases HEI by $19 \%$ and $17 \%$ for adults and children, respectively (Figure 3.1).

Figure 3.2 shows effects of modern retailers on calorie and nutrient intakes. All effects are positive and statistically significant. After controlling for other factors, a 10 percentage point increase in the modern retail expenditure share raises calorie intakes by 133 and $97 \mathrm{kcal} / \mathrm{day}$ for adults and children, respectively. For adults, the additional calorie intake probably also explains the increase in BMI through using modern retailers, as shown above.

Modern retailers also have positive effects on nutrient intakes (Figure 3.2). A 10 percentage point increase in the modern retail expenditure share augments adult and child protein, iron, and zinc intakes by 5-7\%; for vitamin $\mathrm{A}$ the increase is about $3 \%$ for both adults and children. These are sizeable effects, especially when considering that users of modern retailers spend $59 \%$ of their total food expenditures in modern retail outlets. Effects on mean micronutrient adequacy ratios are also positive and significant (Tables A3.12 and A3.13 in the Appendix). These findings underline that modern retailers improve adult and child micronutrient nutrition.

Most previous studies on the effects of modern retailers had focused on supermarkets only, not considering hypermarkets, convenience stores, and fast-food restaurants. In order to test whether our results change when only considering supermarkets, we reran the calorie and nutrient intake models with a modified modern retailer variable that only captures the supermarket food expenditure share. These alternative estimates are similar to those shown in Figure 3.2, only that the effect sizes are somewhat smaller (Table A3.14 in the Appendix), as one would expect. We infer that the different types of modern retailers have similar effects. 
A

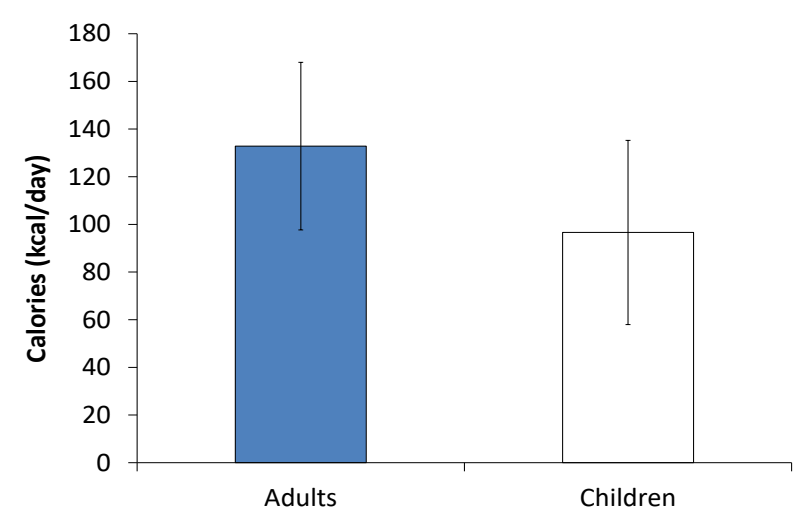

C

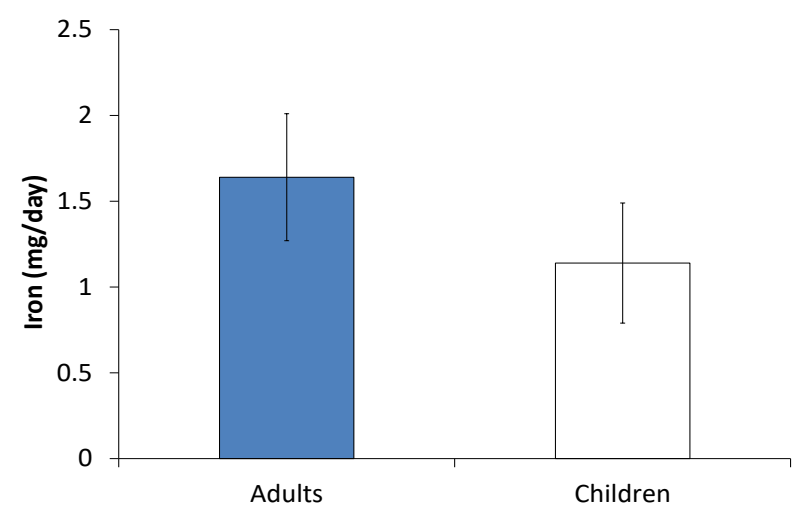

B

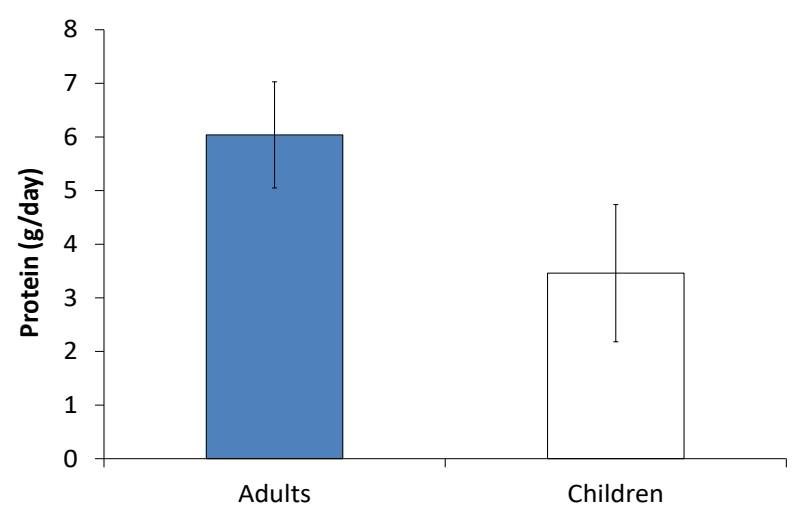

D

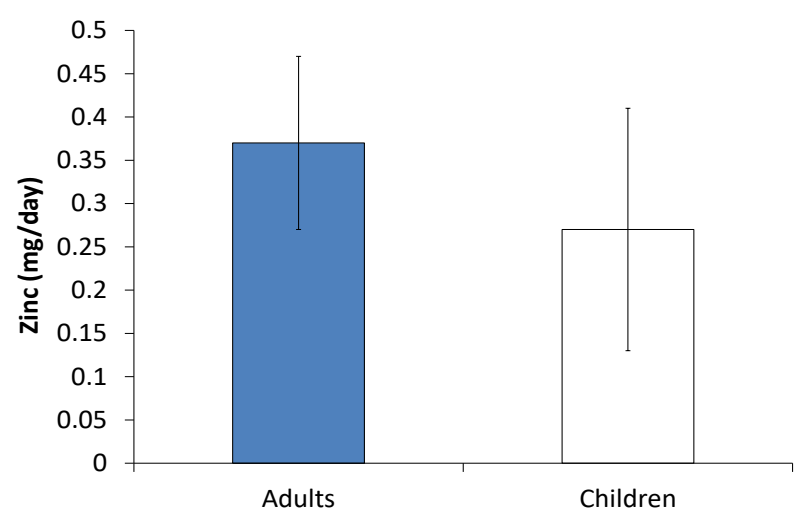

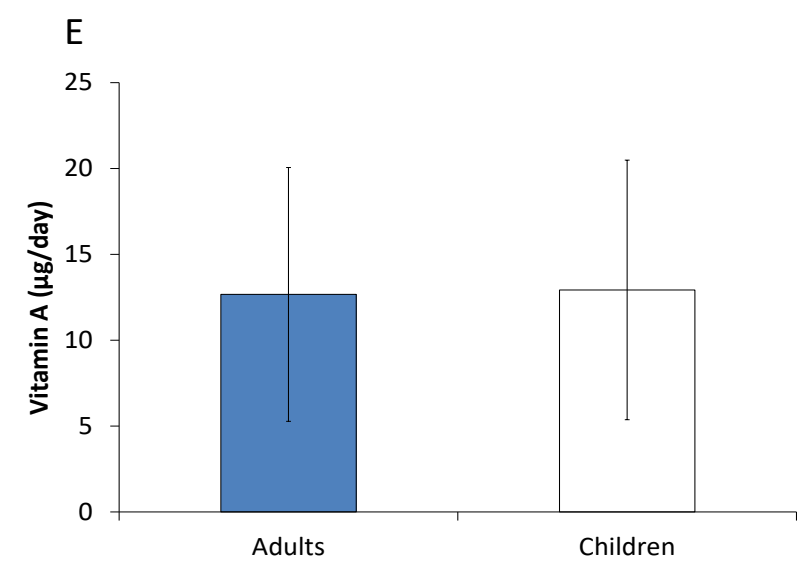

Figure 3.2: Effects of using modern retailers on calorie and nutrient intakes

Effects of a 10 percentage point increase in the household food expenditure share spent in modern retailers are shown with standard errors bars. Effects were estimated with control function models, controlling for income, education, age, and other relevant factors. Models for adults were estimated with 930 individual observations. Models for children were estimated with 499 individual observations. Full model results are shown in Tables A3.10 and A3.11 in the Appendix. (A) Effects on calorie intake in kcal/day. (B) Effects on protein intake in g/day. (C) Effects on iron intake in $\mathrm{mg} /$ day. (D) Effects on zinc intakes in $\mathrm{mg} / \mathrm{day}$. (E) Effects on vitamin A intakes in $\mu \mathrm{g}$ of retinol equivalents per day. 

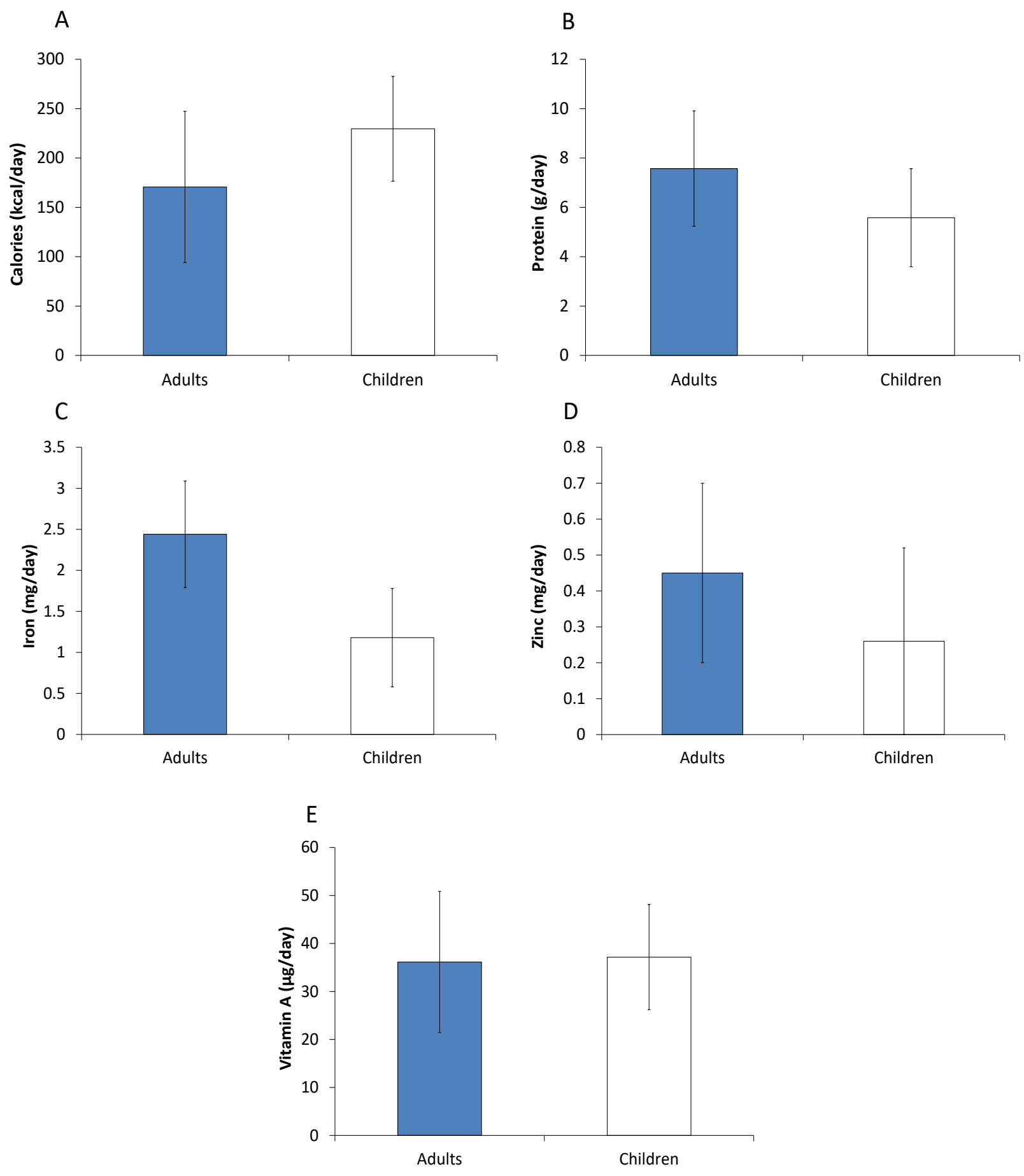

Figure 3.3: Effects of using modern retailers on calorie and nutrient intakes among poor

\section{households}

Effects of a 10 percentage point increase in the household food expenditure share spent in modern retailers are shown with standard errors bars. Effects were estimated with control function models, controlling for income, education, age, and other relevant factors. Models for adults were estimated with 930 individual observations. Models for children were estimated with 499 individual observations. Detailed model results are shown in Table A3.15 in the Appendix. (A) Effects on calorie intake in kcal/day. (B) Effects on protein intake in g/day. (C) Effects on iron intake in $\mathrm{mg} /$ day. (D) Effects on zinc intakes in mg/day. (E) Effects on vitamin A intakes in $\mu \mathrm{g}$ of retinol equivalents per day. 
In additional analyses, we looked specifically at effects on individuals living below the international poverty line of 1.90 US dollars per day. About $24 \%$ of the adults and $35 \%$ of the children in the sample are poor according to this definition. Interestingly, most of the effects of modern retailers on calorie and nutrient intakes are larger for poor individuals (Figure 3.3) than for the full adult and child samples (Figure 3.2). For vitamin A, the positive intake effects on poor individuals are almost three times larger. This is a welcome finding implying that poor people benefit over-proportionally from access to modern retailers. Finally, we disaggregated the adult and child samples by sex. While the estimates are generally less efficient, positive calorie and nutrient intake effects are observed for male and female adults and children (Table A3.16 in the Appendix). The estimated effects of modern retailers on micronutrient intakes are somewhat larger for girls than for boys.

\subsection{Discussion and conclusion}

In Zambia and many other developing countries, food environments are changing rapidly with modern retailers gaining in importance. Most households use both modern and traditional retailers for their food purchases, but the share of the food budget spent in modern retail outlets is rising. According to our data, the average household in Lusaka spends about $42 \%$ of its food budget for purchases in modern retail outlets. Excluding those that only use traditional sources, the modern retail share increases to $59 \%$. Changing food environments can influence people's dietary choices and nutrition. Modern retailers tend to sell more processed foods than traditional markets. Moreover, there are differences in terms of food variety, prices, packaging sizes, and shopping atmosphere. Previous studies suggested that modern retailers increase the consumption of calories from processed foods and therefore contribute to overweight, obesity, and related chronic diseases (Asfaw, 2008; Kimenju et al., 2015; Demmler et al., 2017; Demmler et al., 2018). These studies mostly looked at adult populations. Very few studies analyzed effects of modern retailers on child nutrition, and those that did found mixed results (Umberger et al., 2015; Debela et al., 2020). One shortcoming of all existing studies is that they did not collect individual-level food-intake data. Individual-level data are important to analyze effects of modern retailers on dietary quality and better understand the mechanisms behind the nutrition impacts. 
In this article, we have evaluated dietary and nutrition effects of modern retailers in Lusaka using individual-level food-intake and anthropometric data. The use of modern retailers is positively associated with BMI and the likelihood of being overweight and obese among adults. This is consistent with earlier results for adult populations in Guatemala (Asfaw, 2008) and Kenya (Kimenju et al., 2015; Demmler et al., 2018). For children, we did not find a significant relationship between the use of modern retailers and BMI-for-age Z-scores. Instead, we found that the use of modern retailers increases child height. This is consistent with recent results for children in urban Kenya (Debela et al., 2020). Gains in child height point at likely improvements in dietary quality, even though - due to data limitations - this could not be analyzed in previous studies.

Analysis of our individual-level dietary data confirms that the use of modern retailers improves dietary quality for both adults and children. First, modern retailers contribute to higher calorie intakes, resulting primarily from more consumption of sugar, meat, and dairy products. Second, especially the increase in the consumption of animal source foods also contributes to higher intakes of protein and micronutrients, such as iron, zinc, and vitamin A. These dietary effects are observed for both adults and children. For adults, this implies negative and positive nutrition effects at the same time: the growth of modern retailers is associated with a rise in overweight/obesity and a reduction in micronutrient deficiencies among adults. For children that have not yet reached their final body height, the mechanisms are different. Increases in calorie, protein, and micronutrient intakes contribute to linear growth, especially in situations where child stunting is still commonplace. Recent research with data from a large number of developing countries showed that consumption of animal source foods in particular is positively associated with child linear growth (Headey et al., 2018). This is especially true in situations where regular access to sufficient quantities of plant-based proteins and micronutrients is difficult.

Beyond nutrient intakes, our data from Lusaka show that the use of modern retailers is associated with higher consumption of processed foods (Khonje and Qaim, 2019). This is consistent with findings from other developing countries (Asfaw, 2008; Kelly et al., 2014; Kimenju et al., 2015; Demmler et al., 2018). Processed foods are often considered less healthy than unprocessed foods. However, differentiation is important. Ultra-processed foods with high sugar, fat, and salt, and low micronutrient contents are unhealthy (Popkin 2017; Swinburn et al., 2019; Popkin et al., 2020), whereas the same is not necessarily true for all processed foods. Processing can increase the hygiene and shelf-life of foods and therefore make nutritious, perishable products more accessible 
to consumers. Meat and dairy are good examples. Many poor households rarely buy fresh meat and milk, as these are expensive and highly perishable. Hence, access to processed versions with a longer shelf-life can increase the consumption of nutritious foods. This also explains why the effects of modern retailers on micronutrient intakes of individuals from poor households are particularly large.

A few policy implications emerge from these results. The growth of modern retailers influences people's diets and nutrition, and the effects can be positive and negative. The positive effects on child nutrition and dietary quality of both children and adults are not yet widely appreciated and speak in favor of supporting further modernization of food retail environments. On the other hand, the effect of increasing adult overweight and obesity is undesirable. Regulatory policies that can help to make food environments healthier would be useful. Possible policy interventions include regulation of advertisement and promotional campaigns for unhealthy foods, regulation of health labels and portion/packaging sizes, taxes on ultra-processed foods and beverages with high contents of added sugars, and incentives to offer more healthy foods, among others (Development Initiatives, 2018; Swinburn et al., 2019; Hawkes et al., 2020).

Our results are specific for Lusaka in Zambia and should not be generalized. It is likely that the diet and nutrition effects of modern retailers will be similar in situations where households are relatively poor, child stunting is widespread, and people only have limited or irregular access to healthy foods from traditional markets. This is probably the case in many parts of Africa, as recent research with data from Kenya also suggests (Kimenju et al., 2015; Rischke et al., 2015; Demmler et al., 2018; Debela et al., 2020). However, the effects of modern retailers may be different in situations where consumers are richer, micronutrient deficiencies are not a big problem, and child undernutrition rates are low. One study with data from Indonesia suggested that the use of modern retailers contributes to child overweight in high-income households (Umberger et al., 2015). More research is needed to better understand the diet and nutrition effects of changing food environments in different geographical and socioeconomic contexts. 


\section{Appendix A3}

Table A3.1: Distribution of sampled individuals in Lusaka City

\begin{tabular}{|c|c|c|c|c|c|}
\hline \multirow[b]{2}{*}{ Name of the surveyed compounds/sections } & \multirow{2}{*}{$\begin{array}{l}\text { Income } \\
\text { level }\end{array}$} & \multicolumn{3}{|c|}{ By sex and age cohort } & \multirow[b]{2}{*}{ All } \\
\hline & & $\begin{array}{c}\text { Adult females } \\
\text { ( } \geq 18 \text { years })\end{array}$ & $\begin{array}{c}\text { Adult males } \\
(\geq 18 \text { years })\end{array}$ & $\begin{array}{c}\text { Children/Adolescents } \\
(<18 \text { years })\end{array}$ & \\
\hline $\begin{array}{l}\text { Four: Avondole, Chalala, Kabulonga, and } \\
\text { Woodlands }\end{array}$ & High & 121 & 76 & 85 & 282 \\
\hline Four: Chelston, Chilenje, Kabwata, and PHI & Middle & 250 & 122 & 187 & 559 \\
\hline $\begin{array}{l}\text { Six: Chawama, Chazanga, Gardens, } \\
\text { Kalingalinga, Kaunda Square, and Ng'ombe }\end{array}$ & Low & 252 & 109 & 227 & 588 \\
\hline All & & 623 & 307 & 499 & 1429 \\
\hline
\end{tabular}


Table A3.2: Food groups and components used for construction of dietary quality indicators

\begin{tabular}{|c|c|c|c|}
\hline \multicolumn{4}{|c|}{ Dietary diversity score } \\
\hline Group & Food groups & Food items (examples) & Weight \\
\hline 1 & Cereals and tubers & $\begin{array}{l}\text { Maize, rice, sorghum, millet, bread, } \\
\text { cassava, potatoes, plantains }\end{array}$ & 1 \\
\hline 2 & Pulses & $\begin{array}{l}\text { Beans, cowpea, groundnuts, pigeonpea, } \\
\text { soybean, velvet beans }\end{array}$ & 1 \\
\hline 3 & Meat & $\begin{array}{l}\text { Beef, chicken, ducks, goat meat, sheep } \\
\text { meat, and pork }\end{array}$ & 1 \\
\hline 4 & Fish & Fish (fresh/frozen/dried/tinned) & 1 \\
\hline 5 & Eggs & Eggs & 1 \\
\hline 6 & Vitamin A rich vegetables & Cassava leaves, sweet potato leaves & 1 \\
\hline 7 & Other vegetables & Tomatoes, cabbage etc. & 1 \\
\hline 8 & Fruits & Fruits & 1 \\
\hline 9 & Dairy & Milk, yoghurt, and other dairy products & 1 \\
\hline \multicolumn{4}{|c|}{ Healthy eating index (HEI) } \\
\hline Component & Standard for maximum score & Standard for minimum score (zero) & Maximum points \\
\hline \multicolumn{4}{|l|}{ Adequacy } \\
\hline Total fruits & $\geq 0.8$ cup equivalent per $1000 \mathrm{kcal}$ & No fruit & 5 \\
\hline Whole fruits & $\geq 0 \cdot 4$ cup equivalent per $1000 \mathrm{kcal}$ & No whole fruit & 5 \\
\hline Total vegetables & $\geq 1 \cdot 1$ cup equivalent per $1000 \mathrm{kcal}$ & No vegetables & 5 \\
\hline Greens and beans & $\geq 0 \cdot 2$ cup equivalent per $1000 \mathrm{kcal}$ & No dark-green vegetables or pulses & 5 \\
\hline Whole grains & $\geq 1.5$ ounce equivalent per $1000 \mathrm{kcal}$ & No whole grains & 10 \\
\hline Dairy & $\geq 1 \cdot 3$ cup equivalent per $1000 \mathrm{kcal}$ & No dairy & 10 \\
\hline Total protein foods & $\geq 2.5$ ounce equivalent per $1000 \mathrm{kcal}$ & No protein foods & 5 \\
\hline $\begin{array}{l}\text { Seafood and plant } \\
\text { proteins }\end{array}$ & $\geq 0 \cdot 8$ ounce equivalent per $1000 \mathrm{kcal}$ & No seafood or plant proteins & 5 \\
\hline Fatty acids ${ }^{\text {a }}$ & $(\mathrm{PUFAs}+\mathrm{MUFAs}) / \mathrm{SFAs} \geq 2 \cdot 5$ & $(\mathrm{PUFAs}+\mathrm{MUFAs}) / \mathrm{SFAs} \leq 1 \cdot 2$ & 10 \\
\hline \multicolumn{4}{|l|}{ Moderation } \\
\hline Refined grains & $\leq 1 \cdot 8$ ounce equivalent per $1000 \mathrm{kcal}$ & $\geq 4 \cdot 3$ ounce equivalent per $1000 \mathrm{kcal}$ & 10 \\
\hline Sodium & $\leq 1 \cdot 1$ grams per $1000 \mathrm{kcal}$ & $\geq 2 \cdot 0$ grams per $1000 \mathrm{kcal}$ & 10 \\
\hline Added sugars & $\leq 6.5 \%$ of energy & $\geq 26 \%$ of energy & 10 \\
\hline Saturated fats & $\leq 8 \%$ of energy & $\geq 16 \%$ of energy & 10 \\
\hline
\end{tabular}


Table A3.3: Estimated average requirements of calories and nutrients by sex and age cohort

\begin{tabular}{|c|c|c|c|c|c|c|}
\hline \multirow{3}{*}{ Sex } & \multirow{3}{*}{$\begin{array}{c}\text { Age } \\
\text { (years) }\end{array}$} & \multicolumn{5}{|c|}{ Calorie and nutrients ${ }^{a}$} \\
\hline & & Calorie $^{b}$ & Protein $^{\mathrm{d}}$ & Iron & Zinc $^{\mathrm{e}}$ & Vitamin A \\
\hline & & (kcal/day) & (g/day) & (mg/day) & (mg/day) & ( $\mu$ g retinol/day) \\
\hline \multirow[t]{4}{*}{ Child } & $1-3$ & $531^{\mathrm{c}}$ & $10 \cdot 0$ & $5 \cdot 8$ & $2 \cdot 0$ & 200 \\
\hline & $4-6$ & $595^{\mathrm{c}}$ & $14 \cdot 8$ & $6 \cdot 3$ & $3 \cdot 0$ & 200 \\
\hline & $7-9$ & $680^{\mathrm{c}}$ & $21 \cdot 0$ & $8 \cdot 9$ & $5 \cdot 0$ & 250 \\
\hline & $10-18$ & 2200 & $36 \cdot 4$ & $8 \cdot 0$ & $8 \cdot 6$ & 330 \\
\hline \multirow[t]{3}{*}{ Males } & $19-65$ & 2400 & $55 \cdot 5$ & $11 \cdot 0$ & $10 \cdot 0$ & 300 \\
\hline & $\geq 66$ & 2200 & $54 \cdot 6$ & $8 \cdot 0$ & $10 \cdot 0$ & 300 \\
\hline & $10-18$ & 1600 & $38 \cdot 6$ & $8 \cdot 0$ & $7 \cdot 0$ & 330 \\
\hline \multirow[t]{2}{*}{ Females } & 19-65 non-lactating & 2000 & $54 \cdot 9$ & $18 \cdot 0$ & $6 \cdot 0$ & 270 \\
\hline & $\geq 66$ & 1800 & $57 \cdot 1$ & $8 \cdot 0$ & $6 \cdot 0$ & 300 \\
\hline
\end{tabular}

${ }^{a}$ Estimated average requirements for nutrients are based on FAO (2001), WHO and FAO (2004), and IOM (2006). ${ }^{\mathrm{b}}$ Estimated average energy (calories) requirements for adults are based on IOM (2002), assuming a moderate active individual. ${ }^{\mathrm{c}}$ Daily energy requirements obtained by averaging values for respective age groups from Table $3 \cdot 2$ in FAO (2001). ${ }^{\mathrm{d}}$ Anthropometric data (weight in $\mathrm{kg}$ ) and the reference body weight of $0.8 \mathrm{~g} / \mathrm{kg} /$ day $\left(\mathrm{IOM}, 2002\right.$ ) used as conversion factor to calculate daily protein requirements for individuals. ${ }^{\mathrm{e}}$ Zinc requirements are based in International Zinc Nutrition Consultative Group (IZiNCG) for mixed or refined vegetarian diets (IZiNCG et al., 2004). 
Table A3.4: First-stage estimation results on food purchases in modern retailers

\begin{tabular}{|c|c|c|}
\hline & \multicolumn{2}{|c|}{ Modern retail use (expenditure share, \%) } \\
\hline & Adults & Children \\
\hline & $(1)$ & $(2)$ \\
\hline \multirow[t]{2}{*}{ Male (dummy) } & $-4 \cdot 493 * *$ & $3 \cdot 666$ \\
\hline & $(1 \cdot 958)$ & $(2 \cdot 465)$ \\
\hline \multirow[t]{2}{*}{ Age of respondent (years) } & $-0 \cdot 430$ & $2 \cdot 284 * *$ \\
\hline & $(0 \cdot 312)$ & $(1 \cdot 161)$ \\
\hline \multirow[t]{2}{*}{ Age-squared (years) } & $0 \cdot 005$ & $-0 \cdot 095$ \\
\hline & $(0 \cdot 004)$ & $(0 \cdot 065)$ \\
\hline \multirow[t]{2}{*}{ Education of respondent (years) } & $2 \cdot 611^{* * *}$ & $-0 \cdot 435$ \\
\hline & $(0 \cdot 304)$ & $(0 \cdot 941)$ \\
\hline \multirow[t]{2}{*}{ Household size (individuals) } & $-1 \cdot 870^{* * * *}$ & $-0 \cdot 953$ \\
\hline & $(0 \cdot 561)$ & $(0 \cdot 840)$ \\
\hline \multirow[t]{2}{*}{ Income $(\log )$} & $10 \cdot 992 * * *$ & $12 \cdot 821 * * *$ \\
\hline & $(0 \cdot 835)$ & $(1 \cdot 100)$ \\
\hline \multirow[t]{2}{*}{ Chewa (dummy) } & $1 \cdot 347$ & $-5 \cdot 753$ \\
\hline & $(2 \cdot 682)$ & $(3 \cdot 646)$ \\
\hline \multirow[t]{2}{*}{ Tonga (dummy) } & $-4 \cdot 961 * *$ & $-0 \cdot 331$ \\
\hline & $(2 \cdot 268)$ & $(3 \cdot 459)$ \\
\hline \multirow[t]{2}{*}{ Catholic (dummy) } & $-3 \cdot 751^{*}$ & $-5 \cdot 233^{*}$ \\
\hline & $(2 \cdot 046)$ & $(2 \cdot 936)$ \\
\hline \multicolumn{3}{|l|}{ Instruments } \\
\hline \multirow[t]{2}{*}{ High quality food products (dummy) } & $14 \cdot 463 * * *$ & $16 \cdot 610 * * *$ \\
\hline & $(2 \cdot 370)$ & $(3 \cdot 571)$ \\
\hline \multirow[t]{2}{*}{ Neighbor's shopping mall usage (visits/week) } & $2 \cdot 397 * * *$ & $2 \cdot 146^{* * *}$ \\
\hline & $(0 \cdot 328)$ & $(0 \cdot 447)$ \\
\hline \multirow[t]{2}{*}{ Distance to a shopping mall $(\mathrm{km})$} & $-1 \cdot 132 * * *$ & $-1 \cdot 622 * * *$ \\
\hline & $(0 \cdot 360)$ & $(0 \cdot 493)$ \\
\hline \multirow[t]{2}{*}{ Constant } & $-75 \cdot 621 * * *$ & $-88 \cdot 611 * * *$ \\
\hline & $(10 \cdot 594)$ & $(12 \cdot 721)$ \\
\hline Joint significance of instruments: $\chi^{2}(3)$ & $120^{* * *}$ & $64 * * *$ \\
\hline $\mathrm{N}$ & 930 & 499 \\
\hline
\end{tabular}


Table A3.5: Falsification test for instrument validity (Tobit estimates)

\begin{tabular}{|c|c|c|c|c|c|c|c|}
\hline & $\begin{array}{c}\text { BMI } \\
\left(\mathrm{kg} / \mathrm{m}^{2} / \mathrm{Z} \text {-score }\right)\end{array}$ & $\begin{array}{c}\text { HAZ } \\
\text { (Z-score) }\end{array}$ & $\begin{array}{c}\text { Calorie } \\
\text { (kcal/day) }\end{array}$ & $\begin{array}{l}\text { Protein } \\
\text { (g/day) }\end{array}$ & $\begin{array}{c}\text { Iron } \\
\text { (mg/day) }\end{array}$ & $\begin{array}{c}\text { Zinc } \\
\text { (mg/day) }\end{array}$ & $\begin{array}{c}\text { Vitamin A } \\
(\mu \mathrm{g} \\
\text { retinol/day }) \\
\end{array}$ \\
\hline & $(1)$ & (2) & (3) & (4) & (5) & (6) & $(7)$ \\
\hline Adults & & NA & & & & & \\
\hline \multirow[t]{2}{*}{$\begin{array}{l}\text { High quality food products } \\
\text { (dummy) }\end{array}$} & $-0 \cdot 079$ & & $47 \cdot 512$ & $-0 \cdot 018$ & $-0 \cdot 427$ & $-0 \cdot 073$ & $0 \cdot 674$ \\
\hline & $(0 \cdot 358)$ & & $(96 \cdot 791)$ & $(0 \cdot 302)$ & $(1 \cdot 307)$ & $(0 \cdot 376)$ & $(0 \cdot 696)$ \\
\hline \multirow[t]{2}{*}{$\begin{array}{l}\text { Neighbor's shopping mall } \\
\text { usage (visits/week) }\end{array}$} & $-0 \cdot 004$ & & $24 \cdot 340$ & $0 \cdot 040$ & $0 \cdot 250$ & $0 \cdot 036$ & $-0 \cdot 057$ \\
\hline & $(0 \cdot 051)$ & & $(18 \cdot 738)$ & $(0 \cdot 053)$ & $(0 \cdot 205)$ & $(0 \cdot 058)$ & $(0 \cdot 090)$ \\
\hline \multirow[t]{2}{*}{$\begin{array}{l}\text { Household distance to a } \\
\text { shopping mall }(\mathrm{km})\end{array}$} & $0 \cdot 023$ & & $3 \cdot 231$ & $-0 \cdot 011$ & $0 \cdot 032$ & $-0 \cdot 012$ & $0 \cdot 012$ \\
\hline & $(0 \cdot 052)$ & & $(17 \cdot 223)$ & $(0 \cdot 049)$ & $(0 \cdot 240)$ & $(0 \cdot 040)$ & $(0 \cdot 065)$ \\
\hline Other controls & Yes & & Yes & Yes & Yes & Yes & Yes \\
\hline $\begin{array}{l}\text { Joint significance of } \\
\text { instruments: } F(3,853 / 919)\end{array}$ & $0 \cdot 10$ & & $1 \cdot 02$ & $0 \cdot 47$ & $1 \cdot 02$ & $0 \cdot 36$ & $0 \cdot 35$ \\
\hline $\begin{array}{l}\text { Joint significance of } \\
\text { instruments: } p \text {-value }\end{array}$ & $0 \cdot 958$ & & $0 \cdot 385$ & $0 \cdot 704$ & $0 \cdot 382$ & $0 \cdot 785$ & $0 \cdot 792$ \\
\hline Joint F-statistic & $355^{* * *} *$ & & $16 * * *$ & $3 * * *$ & $16^{* * *}$ & $15^{* * *}$ & $15^{* * *}$ \\
\hline Pseudo- $\mathrm{R}^{2}$ & $0 \cdot 038$ & & $0 \cdot 012$ & $0 \cdot 044$ & $0 \cdot 027$ & $0 \cdot 051$ & $0 \cdot 070$ \\
\hline $\mathrm{N}$ & 864 & & 930 & 930 & 930 & 930 & 930 \\
\hline \multicolumn{8}{|l|}{ Children } \\
\hline \multirow[t]{2}{*}{$\begin{array}{l}\text { High quality food products } \\
\text { (dummy) }\end{array}$} & $-0 \cdot 275$ & $0 \cdot 145$ & $-38 \cdot 936$ & $-0 \cdot 009$ & $1 \cdot 068$ & $-2 \cdot 286$ & $0 \cdot 456$ \\
\hline & $(0 \cdot 441)$ & $(0 \cdot 529)$ & $(157 \cdot 455)$ & $(0 \cdot 302)$ & $(1 \cdot 508)$ & $(2 \cdot 022)$ & $(0 \cdot 692)$ \\
\hline \multirow[t]{2}{*}{$\begin{array}{l}\text { Neighbor's shopping mall } \\
\text { usage (visits/week) }\end{array}$} & $-0 \cdot 124 * *$ & $0 \cdot 159 *$ & $31 \cdot 934$ & $0 \cdot 080^{*}$ & $0 \cdot 126$ & $0 \cdot 343 *$ & 0.036 \\
\hline & $(0 \cdot 062)$ & $(0 \cdot 083)$ & $(21 \cdot 533)$ & $(0 \cdot 045)$ & $(0 \cdot 143)$ & $(0 \cdot 191)$ & $(0 \cdot 076)$ \\
\hline \multirow[t]{2}{*}{$\begin{array}{l}\text { Household distance to a } \\
\text { shopping mall }(\mathrm{km})\end{array}$} & $-0 \cdot 080$ & $-0 \cdot 038$ & -6.909 & $-0 \cdot 025$ & $-0 \cdot 067$ & $0 \cdot 091$ & $-0 \cdot 034$ \\
\hline & $(0 \cdot 054)$ & $(0 \cdot 067)$ & $(15 \cdot 427)$ & $(0 \cdot 033)$ & $(0 \cdot 131)$ & $(0 \cdot 179)$ & $(0 \cdot 054)$ \\
\hline Other controls & Yes & Yes & Yes & Yes & Yes & Yes & Yes \\
\hline $\begin{array}{l}\text { Joint significance of } \\
\text { instruments: } F(3,449 / 461 / 488)\end{array}$ & $1 \cdot 66$ & $1 \cdot 23$ & $0 \cdot 99$ & $1 \cdot 53$ & $0 \cdot 46$ & $1 \cdot 73$ & $0 \cdot 47$ \\
\hline $\begin{array}{l}\text { Joint significance of } \\
\text { instruments: } p \text {-value }\end{array}$ & $0 \cdot 18$ & $0 \cdot 298$ & $0 \cdot 398$ & $0 \cdot 205$ & $0 \cdot 712$ & $0 \cdot 159$ & $0 \cdot 704$ \\
\hline Joint F-statistic & $355^{* * * *}$ & & $145^{* * * *}$ & $202 * * *$ & $48 * * *$ & $3 * * *$ & $39 * * *$ \\
\hline Pseudo- $\mathrm{R}^{2}$ & $0 \cdot 038$ & $0 \cdot 165$ & $0 \cdot 050$ & $0 \cdot 172$ & $0 \cdot 139$ & $0 \cdot 044$ & $0 \cdot 061$ \\
\hline $\mathrm{N}$ & 460 & 472 & 499 & 499 & 499 & 499 & 499 \\
\hline
\end{tabular}

NA, not applicable. Protein and vitamin A intakes were transformed using an inverse hyperbolic sine transformation: $\ln \left[\left\{y+\left(y^{2}+1\right)^{0.5}\right\}\right]$, in order to retain zero-valued observations. Coefficient estimates are shown with robust standard errors clustered at compound level in parentheses. Other controls: age, age-squared, education, household size, log of income, a dummy $(1,0)$ variable for male, ethnic groups - Chewa, and Tonga, and religion status - Catholic. ${ }^{*} \mathrm{p}<0 \cdot 10,{ }^{* * *} \mathrm{p}<0 \cdot 01$ 
Table A3.6: Additional descriptive statistics for users and non-users of modern retailers

\begin{tabular}{|c|c|c|c|c|c|}
\hline \multirow{3}{*}{ Variables } & \multirow{3}{*}{ Units } & \multirow{2}{*}{\multicolumn{2}{|c|}{$\begin{array}{c}\text { Adults }(\geq 18 \text { years) } \\
\text { Modern retailers }\end{array}$}} & \multirow{2}{*}{\multicolumn{2}{|c|}{$\begin{array}{c}\text { Children }(<18 \text { years }) \\
\text { Modern retailers }\end{array}$}} \\
\hline & & & & & \\
\hline & & $\begin{array}{c}\text { Users } \\
(\mathrm{N}=713)\end{array}$ & $\begin{array}{c}\text { Non-users } \\
(\mathrm{N}=217)\end{array}$ & $\begin{array}{c}\text { Users } \\
(\mathrm{N}=358)\end{array}$ & $\begin{array}{c}\text { Non-users } \\
(\mathrm{N}=141)\end{array}$ \\
\hline \multicolumn{6}{|l|}{ Dependent variables } \\
\hline Mean adequacy ratio & Percent, $\%$ & $\begin{array}{l}91 \cdot 51^{* * *} \\
(17 \cdot 68)\end{array}$ & $\begin{array}{c}86.99 \\
(21 \cdot 51)\end{array}$ & $\begin{array}{c}93.44 \\
(16 \cdot 03)\end{array}$ & $\begin{array}{l}92 \cdot 22 \\
(17 \cdot 92)\end{array}$ \\
\hline Iron & Percent, $\%$ & $\begin{array}{c}171 \cdot 96 \\
(103 \cdot 61)\end{array}$ & $\begin{array}{c}175 \cdot 87 \\
(101 \cdot 25)\end{array}$ & $\begin{array}{c}227 \cdot 98 \\
(122 \cdot 89)\end{array}$ & $\begin{array}{c}245 \cdot 23 \\
(157 \cdot 20)\end{array}$ \\
\hline Zinc & Percent, \% & $\begin{array}{l}108 \cdot 69 \\
(85 \cdot 02)\end{array}$ & $\begin{array}{l}108 \cdot 93 \\
(84 \cdot 95)\end{array}$ & $\begin{array}{l}116 \cdot 23 \\
(77 \cdot 49)\end{array}$ & $\begin{array}{c}119 \cdot 10 \\
(136 \cdot 35)\end{array}$ \\
\hline Vitamin A & Percent, \% & $\begin{array}{c}185 \cdot 79 * * * \\
(176 \cdot 86)\end{array}$ & $\begin{array}{c}146 \cdot 75 \\
(165 \cdot 23)\end{array}$ & $\begin{array}{l}185 \cdot 80^{*} \\
(202 \cdot 58)\end{array}$ & $\begin{array}{c}150 \cdot 76 \\
(176 \cdot 71)\end{array}$ \\
\hline \multicolumn{6}{|l|}{ Treatment variable } \\
\hline Modern retailer use & Expenditure share, $\%$ & $\begin{array}{c}59 \cdot 30 * * * \\
(31 \cdot 13)\end{array}$ & $\begin{array}{c}0 \cdot 00 \\
(0 \cdot 00)\end{array}$ & $\begin{array}{l}51 \cdot 59 * * * \\
(30 \cdot 49)\end{array}$ & $\begin{array}{c}0 \cdot 00 \\
(0 \cdot 00)\end{array}$ \\
\hline \multicolumn{6}{|l|}{ Independent variables } \\
\hline Household income & US\$/capita/year & $\begin{array}{l}3265 \cdot 18 * * * \\
(3306 \cdot 72)\end{array}$ & $\begin{array}{c}1041 \cdot 53 \\
(1212 \cdot 42)\end{array}$ & $\begin{array}{l}2575 \cdot 76 * * * \\
(3454 \cdot 14)\end{array}$ & $\begin{array}{c}866 \cdot 82 \\
(1141 \cdot 88)\end{array}$ \\
\hline Male & $1=$ Yes, 0 otherwise & $\begin{array}{c}0 \cdot 33 \\
(0 \cdot 47)\end{array}$ & $\begin{array}{c}0 \cdot 33 \\
(0 \cdot 47)\end{array}$ & $\begin{array}{c}0 \cdot 46 \\
(0 \cdot 50)\end{array}$ & $\begin{array}{c}0 \cdot 45 \\
(0 \cdot 50)\end{array}$ \\
\hline Age of household respondent & Years & $\begin{array}{c}34 \cdot 84 \\
(13 \cdot 97)\end{array}$ & $\begin{array}{c}36 \cdot 56 \\
(15 \cdot 08)\end{array}$ & $\begin{array}{c}8 \cdot 98 \\
(4 \cdot 87)\end{array}$ & $\begin{array}{c}8 \cdot 59 \\
(4 \cdot 73)\end{array}$ \\
\hline Education of respondent & Schooling years & $\begin{array}{c}12 \cdot 27 * * * \\
(3 \cdot 18)\end{array}$ & $\begin{array}{c}9 \cdot 32 \\
(3 \cdot 39)\end{array}$ & $\begin{array}{l}4 \cdot 07^{* *} \\
(3 \cdot 93)\end{array}$ & $\begin{array}{c}3 \cdot 29 \\
(3 \cdot 39)\end{array}$ \\
\hline Household size & Number of members & $\begin{array}{c}4 \cdot 47 * * * \\
(1 \cdot 60)\end{array}$ & $\begin{array}{c}4 \cdot 89 \\
(1 \cdot 89)\end{array}$ & $\begin{array}{c}4.93 \\
(1 \cdot 45)\end{array}$ & $\begin{array}{c}5 \cdot 16 \\
(1 \cdot 60)\end{array}$ \\
\hline Bemba as ethnicity & $1=$ Yes, 0 otherwise & $\begin{array}{c}0 \cdot 29 * * \\
(0 \cdot 45)\end{array}$ & $\begin{array}{c}0 \cdot 20 \\
(0 \cdot 40)\end{array}$ & $\begin{array}{l}0 \cdot 34 * \\
(0 \cdot 47)\end{array}$ & $\begin{array}{c}0 \cdot 26 \\
(0 \cdot 44)\end{array}$ \\
\hline Chewa as ethnicity & $1=$ Yes, 0 otherwise & $\begin{array}{l}0 \cdot 12 * * \\
(0 \cdot 33)\end{array}$ & $\begin{array}{c}0 \cdot 18 \\
(0 \cdot 38)\end{array}$ & $\begin{array}{l}0 \cdot 12 * * \\
(0 \cdot 32)\end{array}$ & $\begin{array}{c}0 \cdot 18 \\
(0 \cdot 39)\end{array}$ \\
\hline Tonga as ethnicity & $1=$ Yes, 0 otherwise & $\begin{array}{c}0 \cdot 21 \\
(0 \cdot 41)\end{array}$ & $\begin{array}{c}0 \cdot 20 \\
(0 \cdot 40)\end{array}$ & $\begin{array}{c}0 \cdot 15 \\
(0 \cdot 35)\end{array}$ & $\begin{array}{c}0 \cdot 17 \\
(0 \cdot 38)\end{array}$ \\
\hline Protestant as a religion & $1=$ Yes, 0 otherwise & $\begin{array}{c}0 \cdot 44 \\
(0 \cdot 50)\end{array}$ & $\begin{array}{c}0 \cdot 41 \\
(0 \cdot 49)\end{array}$ & $\begin{array}{c}0.49 \\
(0 \cdot 50)\end{array}$ & $\begin{array}{c}0 \cdot 45 \\
(0 \cdot 50)\end{array}$ \\
\hline Catholic as a religion & $1=$ Yes, 0 otherwise & $\begin{array}{l}0 \cdot 25^{* * *} \\
(0 \cdot 43)\end{array}$ & $\begin{array}{c}0 \cdot 32 \\
(0 \cdot 47)\end{array}$ & $\begin{array}{c}0 \cdot 18^{* * * *} \\
(0 \cdot 39)\end{array}$ & $\begin{array}{c}0 \cdot 33 \\
(0 \cdot 47)\end{array}$ \\
\hline Physical activity ratio & Ratio; range $(0 \cdot 03-15.95)$ & $\begin{array}{c}2 \cdot 74 \\
(1.45)\end{array}$ & $\begin{array}{c}2 \cdot 76 \\
(1 \cdot 71)\end{array}$ & $\begin{array}{c}3 \cdot 52 \\
(2 \cdot 26)\end{array}$ & $\begin{array}{c}3 \cdot 58 \\
(2 \cdot 35)\end{array}$ \\
\hline Piped or tap drinking water & $1=$ Yes, 0 otherwise & $\begin{array}{l}0 \cdot 94 * * \\
(0 \cdot 24)\end{array}$ & $\begin{array}{c}0 \cdot 88 \\
(0 \cdot 32)\end{array}$ & $\begin{array}{l}0.92 * \\
(0 \cdot 27)\end{array}$ & $\begin{array}{c}0 \cdot 87 \\
(0 \cdot 34)\end{array}$ \\
\hline Non-chronic infections & $1=$ Yes, 0 otherwise & $\begin{array}{c}0 \cdot 27 \\
(0 \cdot 44)\end{array}$ & $\begin{array}{c}0 \cdot 26 \\
(0 \cdot 44)\end{array}$ & $\begin{array}{l}0 \cdot 27 * \\
(0 \cdot 45)\end{array}$ & $\begin{array}{c}0 \cdot 19 \\
(0 \cdot 39)\end{array}$ \\
\hline Distance to the nearest hospital & $\mathrm{km}$; range $(0 \cdot 01-30)$ & $\begin{array}{c}2 \cdot 38 * * * \\
(2 \cdot 06)\end{array}$ & $\begin{array}{c}1.90 \\
(1 \cdot 81)\end{array}$ & $\begin{array}{c}2 \cdot 32 \\
(2 \cdot 01)\end{array}$ & $\begin{array}{c}2.07 \\
(3 \cdot 00)\end{array}$ \\
\hline High quality food products & $1=$ Yes, 0 otherwise & $\begin{array}{c}0 \cdot 23^{* * *} \\
(0 \cdot 42)\end{array}$ & $\begin{array}{c}0 \cdot 01 \\
(0 \cdot 12)\end{array}$ & $\begin{array}{c}0 \cdot 18^{* * * *} \\
(0 \cdot 38)\end{array}$ & $\begin{array}{c}0 \cdot 04 \\
(0 \cdot 19)\end{array}$ \\
\hline Neighbor's shopping mall usage & Number of visits per week & $\begin{array}{c}2 \cdot 88^{* * *} \\
(2 \cdot 91)\end{array}$ & $\begin{array}{c}1 \cdot 68 \\
(2 \cdot 25)\end{array}$ & $\begin{array}{c}3 \cdot 00 * * * \\
(3 \cdot 04)\end{array}$ & $\begin{array}{c}1 \cdot 48 \\
(2 \cdot 31)\end{array}$ \\
\hline $\begin{array}{l}\text { Household distance to a } \\
\text { shopping mall }\end{array}$ & GPS-measured distance in $\mathrm{km}$ & $\begin{array}{l}2 \cdot 57^{* * * *} \\
(2 \cdot 57)\end{array}$ & $\begin{array}{l}3 \cdot 59 \\
(2 \cdot 26)\end{array}$ & $\begin{array}{c}2 \cdot 53^{* * * *} \\
(2 \cdot 50)\end{array}$ & $\begin{array}{l}3 \cdot 88 \\
(2 \cdot 49)\end{array}$ \\
\hline
\end{tabular}

Mean values are shown with standard deviations in parentheses. The average exchange rate was ZMW 9.87 = US\$ 1 in mid-2018. t-tests were used to test for mean differences between users and non-users of modern retailers. ${ }^{*} \mathrm{p}<0.10,{ }^{* * *} \mathrm{p}<0.05,{ }^{* * *} \mathrm{p}<0.01$. 
Table A3.7: Effects of modern retailers on nutritional status (full model results for Table 3.3)

\begin{tabular}{|c|c|c|c|c|c|c|c|c|c|c|}
\hline & \multicolumn{2}{|c|}{ Adults ( $\geq 18$ years) } & \multicolumn{3}{|c|}{ Children $(<18$ years $)$} & \multicolumn{2}{|c|}{ Adults $(\geq 18$ years) } & \multicolumn{3}{|c|}{ Children (<18 years) } \\
\hline & $\begin{array}{c}\text { BMI } \\
\text { OLS } \\
(1)\end{array}$ & $\begin{array}{c}\begin{array}{c}\text { Overweight } \\
\text { /Obese } \\
\text { Probit }\end{array} \\
(2) \\
\end{array}$ & $\frac{\text { OLS }}{(3)}$ & $\begin{array}{c}\begin{array}{c}\text { Overweight } \\
\text { /Obese } \\
\text { Probit }\end{array} \\
(4) \\
\end{array}$ & $\begin{array}{c}\text { OLS } \\
(5)\end{array}$ & $\begin{array}{l}\text { CF } \\
(6)\end{array}$ & $\begin{array}{c}\text { Overweight } \\
\text { /Obese } \\
\text { CF } \\
(7) \\
\end{array}$ & $\begin{array}{l}\mathrm{CF} \\
(8)\end{array}$ & 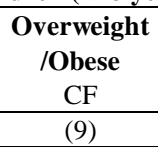 & $\begin{array}{c}\text { CF } \\
(10)\end{array}$ \\
\hline $\begin{array}{l}\text { Modern retail use } \\
\text { (expenditure share, \%) }\end{array}$ & $\begin{array}{c}0 \cdot 012^{* *} \\
(0 \cdot 005)\end{array}$ & $\begin{array}{c}0 \cdot 004 * * * \\
-0.002\end{array}$ & $\begin{array}{c}0 \cdot 001 \\
(0 \cdot 002)\end{array}$ & $\begin{array}{l}-0 \cdot 002 \\
(0 \cdot 004)\end{array}$ & $\begin{array}{l}0 \cdot 006^{* *} \\
(0 \cdot 003)\end{array}$ & $\begin{array}{c}0.004 \\
(0 \cdot 014)\end{array}$ & $\begin{array}{c}0.003 \\
(0.005)\end{array}$ & $\begin{array}{l}-0 \cdot 011 \\
(0 \cdot 008)\end{array}$ & $\begin{array}{c}-0 \cdot 016^{* *} \\
(0 \cdot 007)\end{array}$ & $\begin{array}{c}0 \cdot 026^{* * * *} \\
(0.008)\end{array}$ \\
\hline Male (dummy) & $\begin{array}{c}-0.900 * * \\
(0.349)\end{array}$ & $\begin{array}{l}-0 \cdot 284^{* * * *} \\
(0 \cdot 104)\end{array}$ & $\begin{array}{c}0.558^{* *} \\
(0 \cdot 217)\end{array}$ & $\begin{array}{l}0.410^{*} \\
(0.228)\end{array}$ & $\begin{array}{l}-0.083 \\
(0.186)\end{array}$ & $\begin{array}{c}-0 \cdot 930 * * * \\
(0 \cdot 341)\end{array}$ & $\begin{array}{c}-0 \cdot 290^{* *} \\
(0 \cdot 117)\end{array}$ & $\begin{array}{c}0 \cdot 566 * * * \\
(0 \cdot 190)\end{array}$ & $\begin{array}{l}0 \cdot 440 * * \\
(0 \cdot 179)\end{array}$ & $\begin{array}{l}-0.097 \\
(0.206)\end{array}$ \\
\hline Age (years) & $\begin{array}{c}0 \cdot 374 * * * \\
(0 \cdot 062)\end{array}$ & $\begin{array}{c}0 \cdot 101^{* * * *} \\
(0 \cdot 022)\end{array}$ & $\begin{array}{c}-0 \cdot 154 * * \\
(0 \cdot 067)\end{array}$ & $\begin{array}{l}-0 \cdot 144 \\
(0 \cdot 094)\end{array}$ & $\begin{array}{l}0 \cdot 273^{* * * *} \\
(0 \cdot 063)\end{array}$ & $\begin{array}{l}0 \cdot 371 * * * \\
(0.054)\end{array}$ & $\begin{array}{l}0 \cdot 101^{* * * *} \\
(0 \cdot 016)\end{array}$ & $\begin{array}{c}-0.132^{*} \\
(0.074)\end{array}$ & $\begin{array}{l}-0 \cdot 116 \\
(0 \cdot 076)\end{array}$ & $\begin{array}{c}0 \cdot 236^{* * * *} \\
(0 \cdot 077)\end{array}$ \\
\hline Age-squared (years) & $\begin{array}{c}-0.003 * * * \\
(0.001)\end{array}$ & $\begin{array}{l}-0 \cdot 001 * * * * \\
(0 \cdot 0002)\end{array}$ & $\begin{array}{c}0.003 \\
(0.003)\end{array}$ & $\begin{array}{c}0.003 \\
(0.004)\end{array}$ & $\begin{array}{l}-0.022 * * * \\
\quad(0.002)\end{array}$ & $\begin{array}{l}-0.003 * * * \\
\quad(0 \cdot 001)\end{array}$ & $\begin{array}{l}-0.001 * * * \\
\quad(0.000)\end{array}$ & $\begin{array}{c}0.002 \\
(0.004)\end{array}$ & $\begin{array}{c}0.002 \\
(0.004)\end{array}$ & $\begin{array}{c}-0 \cdot 020^{* * * *} \\
(0.004)\end{array}$ \\
\hline Education (years) & $\begin{array}{l}-0.099 \\
(0.080)\end{array}$ & $\begin{array}{c}-0.0003 \\
(0 \cdot 017)\end{array}$ & $\begin{array}{l}0 \cdot 127^{* *} \\
(0 \cdot 045)\end{array}$ & $\begin{array}{c}0 \cdot 111^{* *} \\
(0 \cdot 050)\end{array}$ & $\begin{array}{c}0 \cdot 114 * * \\
(0 \cdot 053)\end{array}$ & $\begin{array}{l}-0.077 \\
(0.070)\end{array}$ & $\begin{array}{c}0.004 \\
(0.023)\end{array}$ & $\begin{array}{c}0 \cdot 130 * * * \\
(0 \cdot 040)\end{array}$ & $\begin{array}{l}0 \cdot 113^{*} \\
(0 \cdot 060)\end{array}$ & $\begin{array}{l}0 \cdot 109 * * \\
(0 \cdot 049)\end{array}$ \\
\hline Household size (individuals) & $\begin{array}{l}0.086 \\
(0 \cdot 073)\end{array}$ & $\begin{array}{c}0.020 \\
(0 \cdot 030)\end{array}$ & $\begin{array}{l}-0 \cdot 022 \\
(0 \cdot 041)\end{array}$ & $\begin{array}{l}-0.036 \\
(0.049)\end{array}$ & $\begin{array}{l}0.005 \\
(0.047)\end{array}$ & $\begin{array}{l}0 \cdot 071 \\
(0 \cdot 104)\end{array}$ & $\begin{array}{c}0.017 \\
(0.029)\end{array}$ & $\begin{array}{l}-0 \cdot 038 \\
(0 \cdot 047)\end{array}$ & $\begin{array}{l}-0 \cdot 058 \\
(0 \cdot 052)\end{array}$ & $\begin{array}{c}0.029 \\
(0 \cdot 047)\end{array}$ \\
\hline Income $(\log )$ & $\begin{array}{l}0 \cdot 079 \\
(0 \cdot 206)\end{array}$ & $\begin{array}{l}0 \cdot 039 \\
(0 \cdot 056)\end{array}$ & $\begin{array}{l}0 \cdot 025 \\
(0 \cdot 112)\end{array}$ & $\begin{array}{c}0.064 \\
(0.094)\end{array}$ & $\begin{array}{l}-0.055 \\
(0.066)\end{array}$ & $\begin{array}{c}0.172 \\
(0 \cdot 247)\end{array}$ & $\begin{array}{c}0.056 \\
(0.077)\end{array}$ & $\begin{array}{c}0.205 \\
(0 \cdot 146)\end{array}$ & $\begin{array}{c}0.283^{* *} \\
(0 \cdot 127)\end{array}$ & $\begin{array}{c}-0 \cdot 365^{* * *} \\
(0 \cdot 140)\end{array}$ \\
\hline Chewa (dummy) & $\begin{array}{c}0.061 \\
(0.555)\end{array}$ & $\begin{array}{c}0 \cdot 278 \\
(0 \cdot 183)\end{array}$ & $\begin{array}{l}-0 \cdot 220 \\
(0 \cdot 182)\end{array}$ & $\begin{array}{c}-0.315^{*} \\
(0.165)\end{array}$ & $\begin{array}{l}-0.085 \\
(0.136)\end{array}$ & $\begin{array}{c}0 \cdot 061 \\
(0.412)\end{array}$ & $\begin{array}{c}0.278^{* * *} \\
(0.134)\end{array}$ & $\begin{array}{l}-0.263 \\
(0.214)\end{array}$ & $\begin{array}{l}-0 \cdot 396 \\
(0 \cdot 250)\end{array}$ & $\begin{array}{l}-0 \cdot 006 \\
(0 \cdot 148)\end{array}$ \\
\hline Tonga (dummy) & $\begin{array}{c}0.373 \\
(0.449)\end{array}$ & $\begin{array}{c}0 \cdot 145 \\
(0 \cdot 106)\end{array}$ & $\begin{array}{c}0.088 \\
(0 \cdot 193)\end{array}$ & $\begin{array}{l}-0 \cdot 151 \\
(0 \cdot 211)\end{array}$ & $\begin{array}{c}0.014 \\
(0.236)\end{array}$ & $\begin{array}{c}0.334 \\
(0.404)\end{array}$ & $\begin{array}{c}0.137 \\
(0 \cdot 119)\end{array}$ & $\begin{array}{c}0.092 \\
(0.217)\end{array}$ & $\begin{array}{l}-0 \cdot 152 \\
(0.203)\end{array}$ & $\begin{array}{c}0.015 \\
(0.249)\end{array}$ \\
\hline Catholic (dummy) & $\begin{array}{c}0.387 \\
(0 \cdot 275)\end{array}$ & $\begin{array}{c}0.025 \\
(0.113)\end{array}$ & $\begin{array}{c}0.018 \\
(0 \cdot 174)\end{array}$ & $\begin{array}{c}0.018 \\
(0 \cdot 200)\end{array}$ & $\begin{array}{l}-0 \cdot 105 \\
(0 \cdot 170)\end{array}$ & $\begin{array}{c}0 \cdot 367 \\
(0 \cdot 301)\end{array}$ & $\begin{array}{c}0.022 \\
(0.110)\end{array}$ & $\begin{array}{l}-0.061 \\
(0.158)\end{array}$ & $\begin{array}{l}-0.068 \\
(0.197)\end{array}$ & $\begin{array}{c}0.036 \\
(0.146)\end{array}$ \\
\hline Physical activity ratio & $\begin{array}{c}-0.843 * * * \\
(0.120)\end{array}$ & $\begin{array}{c}-0.215 * * * \\
(0.031)\end{array}$ & $\begin{array}{c}-0.140 * * * \\
(0.038)\end{array}$ & $\begin{array}{c}-0.111 * * \\
(0.052)\end{array}$ & $\begin{array}{c}-0.086^{*} \\
(0.048)\end{array}$ & $\begin{array}{c}-0.840 * * * \\
(0 \cdot 116)\end{array}$ & $\begin{array}{c}-0.215 * * * \\
(0.039)\end{array}$ & $\begin{array}{c}-0.132 * * * \\
(0.036)\end{array}$ & $\begin{array}{c}-0.107 * * \\
(0.043)\end{array}$ & $\begin{array}{c}-0.098^{* *} \\
(0.041)\end{array}$ \\
\hline Piped drinking water (dummy) & & & & & $\begin{array}{c}0.263 \\
(0.335)\end{array}$ & & & & & $\begin{array}{c}0 \cdot 214 \\
(0 \cdot 222)\end{array}$ \\
\hline Non-chronic infections (dummy) & & & & & $\begin{array}{l}-0.239 \\
(0.139)\end{array}$ & & & & & $\begin{array}{l}-0 \cdot 182 \\
(0 \cdot 149)\end{array}$ \\
\hline $\begin{array}{l}\text { Distance to the nearest hospital } \\
(\mathrm{km})\end{array}$ & & & & & $\begin{aligned}-0.039 * \\
(0.021)\end{aligned}$ & & & & & $\begin{array}{l}-0.038^{*} \\
(0.022)\end{array}$ \\
\hline Constant & $\begin{array}{c}19 \cdot 016^{* * * *} \\
(2 \cdot 341)\end{array}$ & $\begin{array}{l}-2 \cdot 420 * * * \\
(0 \cdot 595)\end{array}$ & $\begin{array}{c}0.628 \\
(1 \cdot 068)\end{array}$ & $\begin{array}{l}-0 \cdot 549 \\
(1.096)\end{array}$ & $\begin{array}{l}-0.631 \\
(0 \cdot 807)\end{array}$ & $\begin{array}{c}18 \cdot 353 * * * \\
(2 \cdot 360)\end{array}$ & $\begin{array}{c}-2.548 * * * \\
(0.756)\end{array}$ & $\begin{array}{l}-0 \cdot 613 \\
(1 \cdot 223)\end{array}$ & $\begin{array}{c}-2 \cdot 049 * * \\
(0 \cdot 997)\end{array}$ & $\begin{array}{c}1.527 \\
(1.062)\end{array}$ \\
\hline First-stage residual & & & & & & $\begin{array}{l}0.008 \\
(0 \cdot 015)\end{array}$ & $\begin{array}{l}0.002 \\
(0 \cdot 005)\end{array}$ & $\begin{array}{l}0 \cdot 013^{*} \\
(0.007)\end{array}$ & $\begin{array}{c}0 \cdot 016^{* *} \\
(0 \cdot 007)\end{array}$ & $\begin{array}{c}-0 \cdot 023 * * * \\
(0 \cdot 008)\end{array}$ \\
\hline Joint F-statistic/Wald $\chi^{2}$ & $761 * * *$ & $862 * * *$ & $37 * * *$ & $192 * * *$ & & $327 * * *$ & $162 * * *$ & $66^{* * *}$ & $35 * * *$ & $117 * * *$ \\
\hline $\mathrm{R}^{2} /$ Pseudo- $\mathrm{R}^{2}$ & $0 \cdot 220$ & $0 \cdot 146$ & $0 \cdot 057$ & $0 \cdot 046$ & $0 \cdot 133$ & $0 \cdot 220$ & $0 \cdot 147$ & $0 \cdot 063$ & $0 \cdot 055$ & $0 \cdot 151$ \\
\hline $\mathrm{N}$ & 863 & 863 & 458 & 458 & 472 & 863 & 863 & 458 & 458 & 472 \\
\hline
\end{tabular}

Coefficient estimates are shown with robust, bootstrapped standard errors clustered at compound level in parentheses. OLS, ordinary least-squares estimator. Bemba and Protestant are used as reference group for ethnicity - Chewa and Tonga, and religion status - Catholic, respectively. ${ }^{*} \mathrm{p}<0 \cdot 10,{ }^{* *} \mathrm{p}<0 \cdot 05,{ }^{* * *} \mathrm{p}<0 \cdot 01$. 
Table A3.8: Effects of modern retailers on adult dietary diversity (full model results for

Figure 3.1)

\begin{tabular}{|c|c|c|c|c|c|c|}
\hline & HEI & FVS & DDS & HEI & FVS & DDS \\
\hline & $\begin{array}{c}\text { Score } \\
(0-100)\end{array}$ & $\begin{array}{l}\text { Score } \\
(0-18)\end{array}$ & $\begin{array}{l}\text { Score } \\
(0-9)\end{array}$ & $\begin{array}{c}\text { Score } \\
(0-100)\end{array}$ & $\begin{array}{l}\text { Score } \\
(0-18)\end{array}$ & $\begin{array}{l}\text { Score } \\
(0-9)\end{array}$ \\
\hline & Poisson & Poisson & Poisson & $\mathrm{CF}$ & $\mathrm{CF}$ & $\mathrm{CF}$ \\
\hline & (1) & (2) & (3) & (4) & (5) & (6) \\
\hline \multirow{2}{*}{$\begin{array}{l}\text { Modern retail use } \\
\text { (dummy) }\end{array}$} & $1 \cdot 109^{* * *}$ & $1 \cdot 038$ & $1 \cdot 040$ & $1 \cdot 193 * * *$ & $1 \cdot 102 * *$ & $1 \cdot 118^{* *}$ \\
\hline & $(0 \cdot 039)$ & $(0 \cdot 026)$ & $(0 \cdot 041)$ & $(0 \cdot 041)$ & $(0 \cdot 039)$ & $(0.039)$ \\
\hline \multirow[t]{2}{*}{ Male } & -0.994 & $-0 \cdot 995$ & $1 \cdot 019$ & 1.001 & $1 \cdot 001$ & $1 \cdot 025$ \\
\hline & $(0 \cdot 027)$ & $(0 \cdot 021)$ & $(0 \cdot 027)$ & $(0 \cdot 022)$ & $(0 \cdot 020)$ & $(0 \cdot 024)$ \\
\hline \multirow[t]{2}{*}{ Age } & -0.999 & $1 \cdot 001$ & -0.999 & -0.999 & $1 \cdot 001$ & -0.999 \\
\hline & $(0 \cdot 004)$ & $(0 \cdot 003)$ & $(0 \cdot 003)$ & $(0.004)$ & $(0 \cdot 003)$ & $(0 \cdot 004)$ \\
\hline \multirow[t]{2}{*}{ Age-squared } & $1 \cdot 000$ & -1.000 & $1 \cdot 000$ & $-1 \cdot 000$ & $-1 \cdot 000$ & $1 \cdot 000$ \\
\hline & $(0 \cdot 00005)$ & $(0 \cdot 003)$ & $(0 \cdot 00004)$ & $(0 \cdot 00004)$ & $(0 \cdot 00003)$ & $(0 \cdot 00004)$ \\
\hline \multirow[t]{2}{*}{ Education } & $-0.990^{*}$ & -0.999 & -0.999 & $-0 \cdot 988^{* *}$ & $-0 \cdot 997$ & -0.997 \\
\hline & $(0.005)$ & $(0 \cdot 004)$ & $(0 \cdot 005)$ & $(0.005)$ & $(0 \cdot 003)$ & $(0 \cdot 003)$ \\
\hline \multirow{2}{*}{ Household size } & -0.994 & $1 \cdot 004$ & 1.008 & -0.993 & 1.003 & $1 \cdot 007$ \\
\hline & $(0 \cdot 008)$ & $(0 \cdot 008)$ & $(0 \cdot 006)$ & $(0 \cdot 008)$ & $(0 \cdot 007)$ & $(0 \cdot 007)$ \\
\hline \multirow[t]{2}{*}{ Income } & $1 \cdot 012$ & $1 \cdot 023^{*}$ & $1 \cdot 007$ & 1.003 & $1 \cdot 016$ & -0.998 \\
\hline & $(0 \cdot 016)$ & $(0 \cdot 013)$ & $(0 \cdot 013)$ & $(0 \cdot 011)$ & $(0 \cdot 010)$ & $(0 \cdot 012)$ \\
\hline \multirow[t]{2}{*}{ Chewa } & 1.027 & $1 \cdot 049$ & 1.057 & 1.029 & $1 \cdot 050$ & 1.059 \\
\hline & $(0 \cdot 036)$ & $(0.031)$ & $(0.039)$ & $(0 \cdot 033)$ & $(0 \cdot 034)$ & $(0 \cdot 038)$ \\
\hline \multirow[t]{2}{*}{ Tonga } & $1.078^{* * *}$ & $1 \cdot 049$ & $1 \cdot 024$ & $1.084 * * *$ & $1.054^{* * *}$ & $1 \cdot 030$ \\
\hline & $(0.037)$ & $(0.033)$ & $(0 \cdot 036)$ & $(0 \cdot 026)$ & $(0 \cdot 023)$ & $(0 \cdot 024)$ \\
\hline \multirow[t]{2}{*}{ Catholic } & $1 \cdot 053$ & $1 \cdot 027$ & $1.067^{* *}$ & $1.056^{* *}$ & $1 \cdot 030$ & $1.070 * * *$ \\
\hline & $(0.034)$ & $(0.026)$ & $(0 \cdot 021)$ & $(0 \cdot 028)$ & $(0 \cdot 026)$ & $(0 \cdot 024)$ \\
\hline \multirow[t]{2}{*}{ Constant } & $30 \cdot 469^{* * *}$ & $4 \cdot 845^{* * *}$ & $2 \cdot 777 * * *$ & $32 \cdot 133 * * *$ & $5 \cdot 061 * * *$ & $2.931 * * *$ \\
\hline & $(0.034)$ & $(0 \cdot 699)$ & $(0 \cdot 354)$ & $(4 \cdot 518)$ & $(0.543)$ & $(0 \cdot 391)$ \\
\hline \multirow[t]{2}{*}{ First-stage residual } & & & & $-0.998^{* * *}$ & $-0.998 * * *$ & $-0.998^{* * * *}$ \\
\hline & & & & $(0 \cdot 0004)$ & $(0 \cdot 0004)$ & $(0 \cdot 001)$ \\
\hline Wald $\chi^{2}$ & $123^{* * *}$ & $130 * * *$ & $56 * * *$ & $56^{* * *}$ & $41^{* * *}$ & $43^{* * * *}$ \\
\hline Pseudo- $\mathrm{R}^{2}$ & $0 \cdot 013$ & 0.003 & 0.002 & $0 \cdot 021$ & 0.005 & $0 \cdot 004$ \\
\hline $\mathrm{N}$ & 930 & 930 & 930 & 930 & 930 & 930 \\
\hline
\end{tabular}

Incidence rate ratios are shown with bootstrapped standard errors clustered at compound level in parentheses. For Figure 3.1, incidence ratios and standard errors were converted for percentage interpretation. CF, control function approach; HEI, healthy eating index; FVS, food variety score; DDS, dietary diversity score. Bemba and Protestant are used as a reference group for ethnicity - Chewa and Tonga, and religion status - Catholic, respectively. ${ }^{*} \mathrm{p}<0 \cdot 10,{ }^{* *} \mathrm{p}<0 \cdot 05,{ }^{* * *} \mathrm{p}<0 \cdot 01$. 
Table A3.9: Effects of modern retailers on child dietary diversity (full model results for

Figure 3.1)

\begin{tabular}{|c|c|c|c|c|c|c|}
\hline & HEI & FVS & DDS & HEI & FVS & DDS \\
\hline & $\begin{array}{c}\text { Score } \\
(0-100)\end{array}$ & $\begin{array}{l}\text { Score } \\
(0-18)\end{array}$ & $\begin{array}{l}\text { Score } \\
(0-9)\end{array}$ & $\begin{array}{c}\text { Score } \\
(0-100)\end{array}$ & $\begin{array}{l}\text { Score } \\
(0-18)\end{array}$ & $\begin{array}{l}\text { Score } \\
(0-9)\end{array}$ \\
\hline & Poisson & Poisson & Poisson & $\mathrm{CF}$ & $\mathrm{CF}$ & $\mathrm{CF}$ \\
\hline & (1) & (2) & (3) & (4) & (5) & (6) \\
\hline Modern retail use & $1 \cdot 109 * * *$ & 1.049 & -0.974 & $1 \cdot 165^{* * *}$ & 1.049 & 1.014 \\
\hline (dummy) & $(0 \cdot 033)$ & $(0 \cdot 036)$ & $(0 \cdot 039)$ & $(0 \cdot 053)$ & $(0 \cdot 034)$ & $(0 \cdot 042)$ \\
\hline \multirow{2}{*}{ Male } & $1.075^{* * *}$ & -0.998 & $1 \cdot 054^{*}$ & $1 \cdot 075^{* *}$ & -0.998 & $1 \cdot 054$ \\
\hline & $(0 \cdot 025)$ & $(0 \cdot 019)$ & $(0 \cdot 031)$ & $(0 \cdot 033)$ & $(0 \cdot 025)$ & $(0 \cdot 034)$ \\
\hline \multirow[t]{2}{*}{ Age } & $1 \cdot 061^{* * * *}$ & $1 \cdot 025^{* *}$ & $1 \cdot 032 * *$ & $1 \cdot 061^{* * * *}$ & $1 \cdot 025^{*}$ & $1 \cdot 031$ \\
\hline & $(0 \cdot 021)$ & $(0 \cdot 010)$ & $(0 \cdot 014)$ & $(0 \cdot 018)$ & $(0 \cdot 015)$ & $(0 \cdot 017)$ \\
\hline \multirow[t]{2}{*}{ Age-squared } & $-0.998^{*}$ & -0.999 & -0.999 & $-0 \cdot 998^{* *}$ & -0.999 & -0.999 \\
\hline & $(0 \cdot 001)$ & $(0 \cdot 001)$ & $(0 \cdot 001)$ & $(0 \cdot 001)$ & $(0 \cdot 001)$ & $(0 \cdot 001)$ \\
\hline \multirow[t]{2}{*}{ Education } & -0.997 & -0.983 & -0.980 & -0.996 & -0.983 & $-0.979^{*}$ \\
\hline & $(0 \cdot 018)$ & $(0 \cdot 015)$ & $(0 \cdot 014)$ & $(0 \cdot 012)$ & $(0 \cdot 014)$ & $(0 \cdot 011)$ \\
\hline \multirow[t]{2}{*}{ Household size } & -0.999 & $1.021 * * *$ & $1 \cdot 020^{* *}$ & -0.999 & $1 \cdot 021 * *$ & $1.020^{* *}$ \\
\hline & $(0 \cdot 010)$ & $(0 \cdot 007)$ & $(0 \cdot 009)$ & $(0 \cdot 012)$ & $(0 \cdot 009)$ & $(0 \cdot 010)$ \\
\hline \multirow[t]{2}{*}{ Income } & $1 \cdot 017$ & $1 \cdot 040^{* *}$ & $1 \cdot 030$ & $1 \cdot 010$ & $1 \cdot 040^{* * * *}$ & $1.023 * *$ \\
\hline & $(0 \cdot 020)$ & $(0 \cdot 018)$ & $(0 \cdot 021)$ & $(0 \cdot 013)$ & $(0 \cdot 011)$ & $(0 \cdot 012)$ \\
\hline \multirow[t]{2}{*}{ Chewa } & $1 \cdot 118^{* *}$ & $1 \cdot 097^{*}$ & $1 \cdot 162^{* * * *}$ & $1 \cdot 122 * *$ & $1.097^{* * * *}$ & $1 \cdot 165^{* * * *}$ \\
\hline & $(0 \cdot 060)$ & $(0 \cdot 052)$ & $(0 \cdot 062)$ & $(0 \cdot 051)$ & $(0 \cdot 034)$ & $(0 \cdot 054)$ \\
\hline \multirow[t]{2}{*}{ Tonga } & $1 \cdot 086$ & $1.095 * * *$ & $1 \cdot 068^{* *}$ & $1.092 *$ & $1.095^{* * * *}$ & $1.072 *$ \\
\hline & $(0 \cdot 065)$ & $(0 \cdot 034)$ & $(0 \cdot 031)$ & $(0 \cdot 049)$ & $(0 \cdot 037)$ & $(0 \cdot 043)$ \\
\hline \multirow[t]{2}{*}{ Catholic } & $1 \cdot 055^{* *}$ & $1 \cdot 053^{* *}$ & $1 \cdot 019$ & $1.063^{*}$ & $1.053^{* *}$ & $1 \cdot 025$ \\
\hline & $(0 \cdot 027)$ & $(0 \cdot 026)$ & $(0.032)$ & $(0 \cdot 035)$ & $(0 \cdot 025)$ & $(0.031)$ \\
\hline \multirow[t]{2}{*}{ Constant } & $17 \cdot 303^{* * * *}$ & $3.495 * * *$ & $1.745^{* * * *}$ & $17 \cdot 861 * * *$ & $3 \cdot 493 * * *$ & $1.794 * * *$ \\
\hline & $(3 \cdot 180)$ & $(0 \cdot 510)$ & $(0 \cdot 339)$ & $(2 \cdot 673)$ & $(0 \cdot 440)$ & $(0 \cdot 241)$ \\
\hline \multirow[t]{2}{*}{ First-stage residual } & & & & $-0.999 * *$ & $1 \cdot 000$ & $-0.999 *$ \\
\hline & & & & $(0 \cdot 001)$ & $(0 \cdot 001)$ & $(0.001)$ \\
\hline Wald $\chi^{2}$ & $216^{* * * *}$ & $47 * * *$ & $259^{* * * *}$ & $53 * * *$ & $57 * * *$ & $42 * * *$ \\
\hline Pseudo- $\mathrm{R}^{2}$ & $0 \cdot 040$ & 0.009 & $0 \cdot 007$ & $0 \cdot 043$ & 0.009 & $0 \cdot 008$ \\
\hline $\mathrm{N}$ & 499 & 499 & 499 & 499 & 499 & 499 \\
\hline
\end{tabular}

Incidence rate ratios are shown with bootstrapped standard errors clustered at compound level in parentheses. For Figure 3.1, incidence ratios and standard errors were converted for percentage interpretation. CF, control function approach; HEI, healthy eating index; FVS, food variety score; DDS, dietary diversity score. Bemba and Protestant are used as a reference group for ethnicity - Chewa and Tonga, and religion status - Catholic, respectively. ${ }^{*} \mathrm{p}<0 \cdot 10,{ }^{* *} \mathrm{p}<0 \cdot 05,{ }^{* * *} \mathrm{p}<0 \cdot 01$. 
Table A3.10: Effects of modern retailers on adult calorie and nutrient intakes (full model results for Figure 3.2)

\begin{tabular}{|c|c|c|c|c|c|c|c|c|c|c|}
\hline & $\begin{array}{c}\text { Calorie } \\
\text { (kcal/day) } \\
\text { Tobit }\end{array}$ & $\begin{array}{l}\text { Protein } \\
\text { (g/day) } \\
\text { Tobit }\end{array}$ & $\begin{array}{c}\text { Iron } \\
\text { (mg/day) } \\
\text { Tobit }\end{array}$ & $\begin{array}{c}\text { Zinc } \\
\text { (mg/day) } \\
\text { Tobit }\end{array}$ & $\begin{array}{c}\text { Vitamin A } \\
(\mu \mathrm{g} / \text { day }) \\
\text { Tobit }\end{array}$ & $\begin{array}{c}\text { Calorie } \\
\text { (kcal/day) } \\
\text { CF }\end{array}$ & $\begin{array}{c}\text { Protein } \\
\text { (g/day) } \\
\text { CF }\end{array}$ & $\begin{array}{c}\text { Iron } \\
\text { (mg/day) } \\
\text { CF }\end{array}$ & $\begin{array}{c}\text { Zinc } \\
\text { (mg/day) } \\
\text { CF }\end{array}$ & $\begin{array}{c}\text { Vitamin A } \\
(\mu \mathrm{g} / \text { day }) \\
\mathrm{CF}\end{array}$ \\
\hline & $\begin{array}{c}\text { Tobit } \\
(1)\end{array}$ & $\begin{array}{c}\text { Tobit } \\
(2)\end{array}$ & $\begin{array}{c}\text { Tobit } \\
(3)\end{array}$ & (4) & $\begin{array}{l}\text { Tobit } \\
(5)\end{array}$ & (6) & (7) & (8) & $\begin{array}{l}\text { CF } \\
(9)\end{array}$ & $\frac{\mathrm{CF}}{(10)}$ \\
\hline $\begin{array}{l}\text { Modern retail use } \\
\text { (expenditure share, \%) }\end{array}$ & $\begin{array}{c}3 \cdot 556^{* * *} \\
(0 \cdot 916)\end{array}$ & $\begin{array}{c}0 \cdot 096 * * \\
(0.046)\end{array}$ & $\begin{array}{l}0 \cdot 028 * * \\
(0 \cdot 013)\end{array}$ & $\begin{array}{l}0 \cdot 008 * * \\
(0 \cdot 004)\end{array}$ & $\begin{array}{l}0 \cdot 697^{*} \\
(0 \cdot 409)\end{array}$ & $\begin{array}{c}13 \cdot 286^{* * * *} \\
(3 \cdot 517)\end{array}$ & $\begin{array}{c}0.604 * * * \\
(0 \cdot 099)\end{array}$ & $\begin{array}{c}0 \cdot 164 * * * \\
(0.037)\end{array}$ & $\begin{array}{c}0 \cdot 037 * * * \\
(0 \cdot 010)\end{array}$ & $\begin{array}{l}1 \cdot 267 * \\
(0 \cdot 739)\end{array}$ \\
\hline Male & $\begin{array}{c}269 \cdot 499 * * * \\
(71 \cdot 397)\end{array}$ & $\begin{array}{c}6 \cdot 759 * * * \\
(1 \cdot 470)\end{array}$ & $\begin{array}{c}1.466^{* * * *} \\
(0 \cdot 331)\end{array}$ & $\begin{array}{c}0.666^{* * * *} \\
(0 \cdot 224)\end{array}$ & $\begin{array}{l}-14 \cdot 677 \\
(26 \cdot 302)\end{array}$ & $\begin{array}{c}306 \cdot 203 * * * \\
(77 \cdot 809)\end{array}$ & $\begin{array}{c}8 \cdot 705^{* * * *} \\
(2 \cdot 183)\end{array}$ & $\begin{array}{c}2 \cdot 073 * * * \\
(0 \cdot 674)\end{array}$ & $\begin{array}{c}0.773 * * * \\
(0 \cdot 223)\end{array}$ & $\begin{array}{c}9 \cdot 006 \\
(13 \cdot 645)\end{array}$ \\
\hline Age & $\begin{array}{l}21 \cdot 424 * \\
(12 \cdot 323)\end{array}$ & $\begin{array}{c}0 \cdot 639 \\
(0 \cdot 409)\end{array}$ & $\begin{array}{c}0.029 \\
(0.091)\end{array}$ & $\begin{array}{c}0 \cdot 085^{* *} \\
(0 \cdot 034)\end{array}$ & $\begin{array}{l}-0 \cdot 275 \\
(3 \cdot 036)\end{array}$ & $\begin{array}{c}25 \cdot 303^{* * * *} \\
(9 \cdot 497)\end{array}$ & $\begin{array}{l}0.848^{* *} \\
(0 \cdot 382)\end{array}$ & $\begin{array}{c}0.088 \\
(0 \cdot 092)\end{array}$ & $\begin{array}{c}0.096^{* * * *} \\
(0 \cdot 032)\end{array}$ & $\begin{array}{c}1.795 \\
(2 \cdot 243)\end{array}$ \\
\hline Age-squared & $\begin{array}{c}-0.305 * * \\
(0 \cdot 132)\end{array}$ & $\begin{array}{l}-0 \cdot 008 * \\
(0 \cdot 005)\end{array}$ & $\begin{array}{l}-0.001 \\
(0 \cdot 001)\end{array}$ & $\begin{array}{c}-0 \cdot 001 * * * \\
(0 \cdot 0004)\end{array}$ & $\begin{array}{l}-0 \cdot 009 \\
(0 \cdot 036)\end{array}$ & $\begin{array}{c}-0 \cdot 347 * * * \\
(0 \cdot 106)\end{array}$ & $\begin{array}{c}-0 \cdot 010^{* * *} \\
(0 \cdot 005)\end{array}$ & $\begin{array}{l}-0 \cdot 001 \\
(0 \cdot 001)\end{array}$ & $\begin{array}{c}-0 \cdot 001 * * * \\
(0 \cdot 0004)\end{array}$ & $\begin{array}{l}-0 \cdot 024 \\
(0 \cdot 027)\end{array}$ \\
\hline Education & $\begin{array}{c}-22 \cdot 152 * * * \\
(8 \cdot 361)\end{array}$ & $\begin{array}{c}-0.755^{* * * *} \\
(0 \cdot 244)\end{array}$ & $\begin{array}{l}-0.087 \\
(0 \cdot 074)\end{array}$ & $\begin{array}{c}-0 \cdot 059 * \\
(0 \cdot 033)\end{array}$ & $\begin{array}{c}4 \cdot 750 \\
(2 \cdot 966)\end{array}$ & $\begin{array}{c}-50 \cdot 816^{* * * *} \\
(17 \cdot 861)\end{array}$ & $\begin{array}{c}-2 \cdot 256^{* * * *} \\
(0 \cdot 547)\end{array}$ & $\begin{array}{c}-0 \cdot 480 * * * \\
(0 \cdot 154)\end{array}$ & $\begin{array}{c}-0 \cdot 144 * * * \\
(0 \cdot 053)\end{array}$ & $\begin{array}{l}-0 \cdot 308 \\
(3 \cdot 107)\end{array}$ \\
\hline Household size & $\begin{array}{l}-40 \cdot 738 \\
(36 \cdot 400)\end{array}$ & $\begin{array}{c}-3 \cdot 180 * * \\
(1 \cdot 305)\end{array}$ & $\begin{array}{l}-0 \cdot 388 \\
(0 \cdot 250)\end{array}$ & $\begin{array}{c}-0.253 * * \\
(0 \cdot 117)\end{array}$ & $\begin{array}{c}-14 \cdot 600 * * \\
(5 \cdot 918)\end{array}$ & $\begin{array}{l}-19 \cdot 778 \\
(22 \cdot 973)\end{array}$ & $\begin{array}{c}-2 \cdot 080^{* * *} \\
(0.739)\end{array}$ & $\begin{array}{l}-0 \cdot 100 \\
(0 \cdot 202)\end{array}$ & $\begin{array}{c}-0 \cdot 192 * * * \\
(0 \cdot 072)\end{array}$ & $\begin{array}{c}-7 \cdot 428^{*} \\
(3 \cdot 896)\end{array}$ \\
\hline Income & $\begin{array}{c}45 \cdot 815 \\
(37 \cdot 039)\end{array}$ & $\begin{array}{c}1 \cdot 182 \\
(1 \cdot 035)\end{array}$ & $\begin{array}{c}0 \cdot 205 \\
(0 \cdot 356)\end{array}$ & $\begin{array}{c}0 \cdot 040 \\
(0 \cdot 106)\end{array}$ & $\begin{array}{c}22 \cdot 066 \\
(13 \cdot 680)\end{array}$ & $\begin{array}{l}-76 \cdot 970 \\
(52 \cdot 564)\end{array}$ & $\begin{array}{c}-5 \cdot 222 * * * \\
(1 \cdot 522)\end{array}$ & $\begin{array}{c}-1 \cdot 514 * * * \\
(0 \cdot 542)\end{array}$ & $\begin{array}{c}-0 \cdot 322^{* *} \\
(0 \cdot 158)\end{array}$ & $\begin{array}{c}1.923 \\
(10 \cdot 781)\end{array}$ \\
\hline Chewa & $\begin{array}{c}-178.209^{*} \\
(95 \cdot 287)\end{array}$ & $\begin{array}{c}0.603 \\
(4 \cdot 727)\end{array}$ & $\begin{array}{c}0 \cdot 044 \\
(1 \cdot 063)\end{array}$ & $\begin{array}{l}-0 \cdot 364 \\
(0 \cdot 434)\end{array}$ & $\begin{array}{c}-7 \cdot 026 \\
(21 \cdot 073)\end{array}$ & $\begin{array}{c}-180 \cdot 927 * \\
(100 \cdot 034)\end{array}$ & $\begin{array}{c}0.425 \\
(3 \cdot 692)\end{array}$ & $\begin{array}{l}-0.063 \\
(1 \cdot 029)\end{array}$ & $\begin{array}{l}-0 \cdot 370 \\
(0 \cdot 339)\end{array}$ & $\begin{array}{c}9 \cdot 029 \\
(20 \cdot 210)\end{array}$ \\
\hline Tonga & $\begin{array}{c}162 \cdot 359^{* *} \\
(81 \cdot 023)\end{array}$ & $\begin{array}{c}10 \cdot 934 * * * \\
(2 \cdot 299)\end{array}$ & $\begin{array}{c}2.943 * * * \\
(0.773)\end{array}$ & $\begin{array}{l}0 \cdot 540^{* *} \\
(0 \cdot 269)\end{array}$ & $\begin{array}{c}4.938 \\
(26 \cdot 619)\end{array}$ & $\begin{array}{c}212 \cdot 855^{* *} \\
(96 \cdot 257)\end{array}$ & $\begin{array}{c}13 \cdot 568 * * * \\
(2 \cdot 922)\end{array}$ & $\begin{array}{c}3 \cdot 739 * * * \\
(0.938)\end{array}$ & $\begin{array}{c}0 \cdot 688^{* *} \\
(0 \cdot 325)\end{array}$ & $\begin{array}{c}23 \cdot 457 \\
(17 \cdot 390)\end{array}$ \\
\hline Catholic & $\begin{array}{c}109.489 \\
(71 \cdot 476)\end{array}$ & $\begin{array}{l}7 \cdot 143 * * \\
(2 \cdot 925)\end{array}$ & $\begin{array}{c}1.091 \\
(0.751)\end{array}$ & $\begin{array}{l}0.593^{* *} \\
(0 \cdot 246)\end{array}$ & $\begin{array}{l}72 \cdot 108 * \\
(37 \cdot 249)\end{array}$ & $\begin{array}{c}134 \cdot 599 * \\
(81 \cdot 645)\end{array}$ & $\begin{array}{c}8.469 * * * \\
(2 \cdot 691)\end{array}$ & $\begin{array}{l}1.466^{* *} \\
(0.747)\end{array}$ & $\begin{array}{c}0.667 * * \\
(0.263)\end{array}$ & $\begin{array}{c}41 \cdot 942 * * * \\
(14 \cdot 340)\end{array}$ \\
\hline Constant & $\begin{array}{c}1990.091^{* * * *} \\
(602.489)\end{array}$ & $\begin{array}{c}70 \cdot 085^{* * * *} \\
(16 \cdot 065)\end{array}$ & $\begin{array}{c}17 \cdot 915^{* * * *} \\
(3 \cdot 279)\end{array}$ & $\begin{array}{c}6 \cdot 350^{* * * *} \\
(1 \cdot 622)\end{array}$ & $\begin{array}{c}164 \cdot 583 \\
(149 \cdot 339)\end{array}$ & $\begin{array}{c}2854.905^{* * *} \\
(501 \cdot 210)\end{array}$ & $\begin{array}{c}115 \cdot 080 * * * \\
(15 \cdot 024)\end{array}$ & $\begin{array}{c}29 \cdot 946^{* * *} \\
\quad(4 \cdot 638)\end{array}$ & $\begin{array}{c}8.902 * * * \\
(1.639)\end{array}$ & $\begin{array}{c}190 \cdot 720^{* *} \\
(94 \cdot 957)\end{array}$ \\
\hline First-stage residual & & & & & & $\begin{array}{c}-11 \cdot 004^{* * * *} \\
(3 \cdot 836)\end{array}$ & $\begin{array}{c}-0.574 * * * \\
(0 \cdot 108)\end{array}$ & $\begin{array}{c}-0 \cdot 150 * * * \\
(0.039)\end{array}$ & $\begin{array}{c}-0.033 * * * \\
(0 \cdot 011)\end{array}$ & $\begin{array}{l}-0.637 \\
(0.787)\end{array}$ \\
\hline Joint F-statistic & $11^{* * * *}$ & $202 * * *$ & $43^{* * * *}$ & $8^{* * *}$ & $12 * * *$ & & & & & \\
\hline Wald $\chi^{2}$ & & & & & & $103 * * *$ & $199^{* * *}$ & $78^{* * *}$ & $98 * * *$ & $88 * * *$ \\
\hline Pseudo- $\mathrm{R}^{2}$ & $0 \cdot 004$ & $0 \cdot 010$ & $0 \cdot 024$ & $0 \cdot 011$ & $0 \cdot 006$ & 0.004 & $0 \cdot 013$ & 0.033 & $0 \cdot 012$ & 0.009 \\
\hline $\mathrm{N}$ & 930 & 930 & 930 & 930 & 930 & 930 & 930 & 930 & 930 & 930 \\
\hline
\end{tabular}

Coefficient estimates are shown with bootstrapped standard errors clustered at compound level in parentheses. Estimates for modern retail use can be interpreted as marginal effects of a 1 percentage point increase in the modern retail expenditure share. For Figure 3.2, coefficients and standard errors were multiplied by 10, to show effects of a 10 percentage point increase in the modern retail expenditure share. $\mathrm{CF}$, control function approach. Bemba and Protestant are used as reference group for ethnicity - Chewa and Tonga, and religion status - Catholic, respectively. ${ }^{*} \mathrm{p}<0 \cdot 10,{ }^{* *} \mathrm{p}<0 \cdot 05,{ }^{* * *} \mathrm{p}<0 \cdot 01$. 
Table A3.11: Effects of modern retailers on child calorie and nutrient intakes (full model results for Figure 3.2)

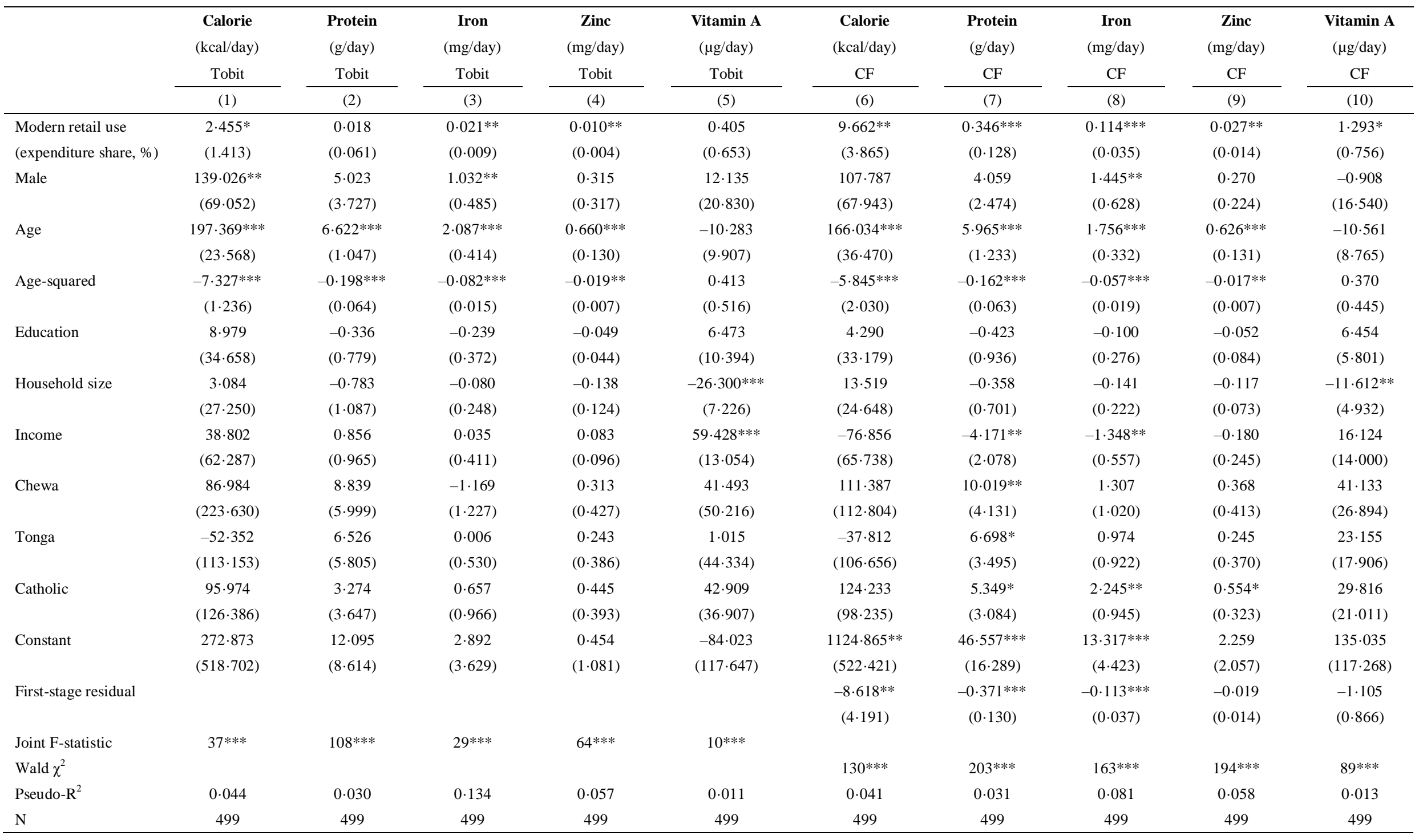

Coefficient estimates are shown with bootstrapped standard errors clustered at compound level in parentheses. Estimates for modern retail use can be interpreted as marginal effects of a 1 percentage point increase in the modern retail expenditure share. For Figure 3.2, coefficients and standard errors were multiplied by 10, to show effects of a 10 percentage point increase in the modern retail expenditure share. $\mathrm{CF}$, control function approach. Bemba and Protestant are used as reference group for ethnicity - Chewa and Tonga, and religion status - Catholic, respectively. ${ }^{*} \mathrm{p}<0 \cdot 10,{ }^{* *} \mathrm{p}<0 \cdot 05,{ }^{* * * *} \mathrm{p}<0 \cdot 01$. 
Table A3.12: Effects of modern retailers on adult micronutrient adequacy ratios

\begin{tabular}{|c|c|c|c|c|}
\hline & \multirow[b]{2}{*}{ Mean adequacy ratio } & \multicolumn{3}{|c|}{ Micronutrient adequacy ratio } \\
\hline & & Iron & Zinc & Vitamin A \\
\hline & $(1)$ & $(2)$ & $(3)$ & (4) \\
\hline Modern retail use & $0 \cdot 245^{* * *}$ & $1 \cdot 073 * * *$ & $0 \cdot 386^{*}$ & $-0 \cdot 033$ \\
\hline (expenditure share, \%) & $(0 \cdot 046)$ & $(0 \cdot 283)$ & $(0 \cdot 230)$ & $(0 \cdot 660)$ \\
\hline \multirow[t]{2}{*}{ Male } & $3 \cdot 739 * * *$ & $91 \cdot 887 * * *$ & $-40 \cdot 047 * * *$ & $-23 \cdot 791^{*}$ \\
\hline & $(1 \cdot 117)$ & $(7 \cdot 163)$ & $(5 \cdot 657)$ & $(12 \cdot 423)$ \\
\hline \multirow[t]{2}{*}{ Age } & $-0 \cdot 344 * *$ & $-4 \cdot 543 * * *$ & $1 \cdot 120$ & $1 \cdot 057$ \\
\hline & $(0 \cdot 171)$ & $(1 \cdot 150)$ & $(0 \cdot 789)$ & $(1 \cdot 988)$ \\
\hline \multirow[t]{2}{*}{ Age-squared } & $0 \cdot 003^{*}$ & $0 \cdot 060 * * *$ & $-0 \cdot 016^{*}$ & $-0 \cdot 020$ \\
\hline & $(0 \cdot 002)$ & $(0 \cdot 014)$ & $(0 \cdot 008)$ & $(0 \cdot 023)$ \\
\hline \multirow[t]{2}{*}{ Education } & $-0.563^{*}$ & $-3 \cdot 450 * *$ & $-1 \cdot 553$ & $3 \cdot 983$ \\
\hline & $(0 \cdot 288)$ & $(1 \cdot 431)$ & $(1 \cdot 199)$ & $(2 \cdot 642)$ \\
\hline \multirow[t]{2}{*}{ Household size } & $-0 \cdot 545$ & $-3 \cdot 071 *$ & $-4 \cdot 171 * * *$ & $-6 \cdot 718^{*}$ \\
\hline & $(0 \cdot 465)$ & $(1 \cdot 752)$ & $(1 \cdot 339)$ & $(3 \cdot 503)$ \\
\hline \multirow[t]{2}{*}{ Income } & $-0 \cdot 688$ & $-10 \cdot 273 * * *$ & $-5 \cdot 625^{*}$ & $15 \cdot 838$ \\
\hline & $(0 \cdot 859)$ & $(3 \cdot 761)$ & $(3 \cdot 340)$ & $(10 \cdot 274)$ \\
\hline \multirow[t]{2}{*}{ Chewa } & $-0 \cdot 827$ & $-3 \cdot 285$ & $-5 \cdot 520$ & $-27 \cdot 278^{*}$ \\
\hline & $(2 \cdot 194)$ & $(8 \cdot 198)$ & $(7 \cdot 358)$ & $(14 \cdot 103)$ \\
\hline \multirow[t]{2}{*}{ Tonga } & $3 \cdot 019 * *$ & $32 \cdot 493 * * *$ & $7 \cdot 424$ & $-2 \cdot 784$ \\
\hline & $(1 \cdot 237)$ & $(8 \cdot 499)$ & $(8 \cdot 037)$ & $(16 \cdot 177)$ \\
\hline \multirow[t]{2}{*}{ Catholic } & $2 \cdot 190$ & $16 \cdot 228 * * *$ & $11 \cdot 188^{*}$ & $34 \cdot 900 * * *$ \\
\hline & $(1 \cdot 389)$ & $(5 \cdot 854)$ & $(5 \cdot 967)$ & $(12 \cdot 633)$ \\
\hline \multirow[t]{2}{*}{ Constant } & $99 \cdot 622 * * *$ & $309.095 * * *$ & $175 \cdot 356 * * *$ & $4 \cdot 742$ \\
\hline & $(8 \cdot 760)$ & $(38 \cdot 222)$ & $(34 \cdot 081)$ & $(90 \cdot 443)$ \\
\hline \multirow[t]{2}{*}{ First-stage residual } & $-0 \cdot 210^{* * *}$ & $-0.997 * * *$ & $-0 \cdot 321$ & $0 \cdot 210$ \\
\hline & $(0 \cdot 050)$ & $(0 \cdot 289)$ & $(0 \cdot 246)$ & $(0 \cdot 718)$ \\
\hline Wald $\chi^{2}(11)$ & $142 * * *$ & $296 * * *$ & $121 * * *$ & $73 * * *$ \\
\hline $\mathrm{R}^{2}$ & $0 \cdot 093$ & $0 \cdot 233$ & $0 \cdot 070$ & $0 \cdot 053$ \\
\hline $\mathrm{N}$ & 930 & 930 & 930 & 930 \\
\hline
\end{tabular}

Coefficient estimates from control function models are shown with bootstrapped standard errors clustered at compound level in parentheses. ${ }^{*} \mathrm{p}<0 \cdot 10,{ }^{* *} \mathrm{p}<0 \cdot 05,{ }^{* * *} \mathrm{p}<0 \cdot 01$. 
Table A3.13: Effects of modern retailers on child micronutrient adequacy ratios

\begin{tabular}{|c|c|c|c|c|}
\hline & \multirow[b]{2}{*}{ Mean adequacy ratio } & \multicolumn{3}{|c|}{ Micronutrient adequacy ratio } \\
\hline & & Iron & Zinc & Vitamin A \\
\hline & $(1)$ & $(2)$ & (3) & (4) \\
\hline Modern retail use & $0 \cdot 179 * * *$ & $1 \cdot 380^{* * *}$ & $0 \cdot 849^{* *}$ & $0 \cdot 072$ \\
\hline (expenditure share, $\%$ ) & $(0 \cdot 069)$ & $(0 \cdot 659)$ & $(0 \cdot 428)$ & $(0 \cdot 945)$ \\
\hline \multirow[t]{2}{*}{ Male } & $2 \cdot 823^{* *}$ & $25 \cdot 477 * *$ & $0 \cdot 283$ & $11 \cdot 346$ \\
\hline & $(1.408)$ & $(12 \cdot 476)$ & $(7 \cdot 283)$ & $(17 \cdot 115)$ \\
\hline \multirow[t]{2}{*}{ Age } & $0 \cdot 800$ & $10 \cdot 011^{*}$ & $-11 \cdot 086 * * *$ & $-17 \cdot 490 *$ \\
\hline & $(0.997)$ & $(5 \cdot 237)$ & $(3 \cdot 748)$ & $(9 \cdot 156)$ \\
\hline \multirow[t]{2}{*}{ Age-squared } & $-0 \cdot 028$ & $-0 \cdot 113$ & $0 \cdot 473^{* * *}$ & $0 \cdot 606$ \\
\hline & $(0 \cdot 043)$ & $(0 \cdot 283)$ & $(0 \cdot 168)$ & $(0 \cdot 460)$ \\
\hline \multirow[t]{2}{*}{ Education } & $-0 \cdot 082$ & -1.419 & $-4 \cdot 091 *$ & $-1 \cdot 268$ \\
\hline & $(0 \cdot 482)$ & $(3 \cdot 613)$ & $(2 \cdot 323)$ & $(4 \cdot 630)$ \\
\hline \multirow[t]{2}{*}{ Household size } & $-0 \cdot 383$ & $-5 \cdot 487^{*}$ & $-2 \cdot 659$ & $-11 \cdot 848^{* *}$ \\
\hline & $(0 \cdot 555)$ & $(3 \cdot 190)$ & $(2 \cdot 119)$ & $(5 \cdot 390)$ \\
\hline \multirow[t]{2}{*}{ Income } & $-0 \cdot 476$ & $-21 \cdot 234 * *$ & $-10 \cdot 676$ & $41 \cdot 194 * *$ \\
\hline & $(1 \cdot 316)$ & $(10 \cdot 194)$ & $(8 \cdot 768)$ & $(16 \cdot 821)$ \\
\hline \multirow[t]{2}{*}{ Chewa } & $-0 \cdot 145$ & $31 \cdot 016$ & $10 \cdot 337$ & $39 \cdot 890$ \\
\hline & $(2 \cdot 119)$ & $(20 \cdot 533)$ & $(11 \cdot 368)$ & $(30 \cdot 429)$ \\
\hline \multirow[t]{2}{*}{ Tonga } & 1.032 & $43 \cdot 547 * *$ & $20 \cdot 892$ & $-8 \cdot 811$ \\
\hline & $(1.669)$ & $(18 \cdot 522)$ & $(18 \cdot 827)$ & $(16 \cdot 828)$ \\
\hline \multirow[t]{2}{*}{ Catholic } & $2 \cdot 497$ & $36 \cdot 843 * * *$ & $13 \cdot 791$ & $5 \cdot 847$ \\
\hline & $(1 \cdot 837)$ & $(14 \cdot 034)$ & $(9 \cdot 011)$ & $(20 \cdot 482)$ \\
\hline \multirow[t]{2}{*}{ Constant } & $86 \cdot 888^{* * *}$ & $303 \cdot 276 * * *$ & $255 \cdot 781 * * *$ & $-61 \cdot 510$ \\
\hline & $(11 \cdot 732)$ & $(79 \cdot 592)$ & $(76 \cdot 754)$ & $(137 \cdot 665)$ \\
\hline \multirow[t]{2}{*}{ First-stage residual } & $-0 \cdot 165 * *$ & $-1.411 * *$ & $-0 \cdot 676$ & $0 \cdot 420$ \\
\hline & $(0 \cdot 075)$ & $(0.682)$ & $(0 \cdot 482)$ & $(1.019)$ \\
\hline Wald $\chi^{2}(11)$ & $38 * * *$ & $92 * * *$ & $69 * * *$ & $78 * * *$ \\
\hline $\mathrm{R}^{2}$ & 0.067 & $0 \cdot 117$ & 0.097 & $0 \cdot 132$ \\
\hline $\mathrm{N}$ & 499 & 499 & 499 & 499 \\
\hline
\end{tabular}

Coefficient estimates from control function models are shown with bootstrapped standard errors clustered at compound level in parentheses. ${ }^{*} \mathrm{p}<0 \cdot 10,{ }^{* * *} \mathrm{p}<0 \cdot 05,{ }^{* * * *} \mathrm{p}<0 \cdot 01$. 
Table A3.14: Effects of supermarkets on child and adult calorie and nutrient intakes

\begin{tabular}{|c|c|c|c|c|c|}
\hline & $\begin{array}{c}\text { Calorie } \\
\text { (kcal/day) }\end{array}$ & $\begin{array}{l}\text { Protein } \\
(\mathrm{g} / \text { day })\end{array}$ & $\begin{array}{c}\text { Iron } \\
\text { (mg/day) }\end{array}$ & $\begin{array}{c}\text { Zinc } \\
\text { (mg/day) }\end{array}$ & $\begin{array}{c}\text { Vitamin A } \\
(\mu \mathrm{g} \text { retinol/day) }\end{array}$ \\
\hline & (1) & (2) & (3) & (4) & (5) \\
\hline \multicolumn{6}{|l|}{ Adults } \\
\hline Supermarket use & $10 \cdot 430 * * *$ & $0.497 * * *$ & $0 \cdot 139 * * *$ & $0 \cdot 029 * * *$ & $1 \cdot 029 * *$ \\
\hline (expenditure share, $\%$ ) & $(2 \cdot 882)$ & $(0 \cdot 094)$ & $(0.031)$ & $(0 \cdot 009)$ & $(0 \cdot 488)$ \\
\hline Controls & Yes & Yes & Yes & Yes & Yes \\
\hline \multirow[t]{2}{*}{ First-stage residual } & $-7 \cdot 724 * *$ & $-0.440^{* * * *}$ & $-0.118^{* * *}$ & $-0.024 * *$ & $-0 \cdot 424$ \\
\hline & $(3 \cdot 237)$ & $(0 \cdot 103)$ & $(0.034)$ & $(0 \cdot 009)$ & $(0.525)$ \\
\hline Wald $\chi^{2}(11)$ & $76^{* * *}$ & $133^{* * * *}$ & $53 * * *$ & $72 * * *$ & $66 * * *$ \\
\hline Pseudo- $\mathrm{R}^{2}$ & $0 \cdot 004$ & 0.013 & 0.034 & $0 \cdot 012$ & 0.009 \\
\hline $\mathrm{N}$ & 930 & 930 & 930 & 930 & 930 \\
\hline \multicolumn{6}{|l|}{ Children } \\
\hline Supermarket use & $8 \cdot 276^{* *}$ & $0 \cdot 295^{* *}$ & $0 \cdot 102^{* * *}$ & $0.022 *$ & $1 \cdot 106$ \\
\hline (expenditure share, \%) & $(3 \cdot 595)$ & $(0 \cdot 119)$ & $(0 \cdot 029)$ & $(0 \cdot 013)$ & $(0.722)$ \\
\hline Controls & Yes & Yes & Yes & Yes & Yes \\
\hline \multirow[t]{2}{*}{ First-stage residual } & $-8 \cdot 810 * *$ & $-0 \cdot 324 * * *$ & $-0 \cdot 104^{* * *}$ & $-0 \cdot 015$ & $-0 \cdot 843$ \\
\hline & $(3 \cdot 700)$ & $(0 \cdot 123)$ & $(0.033)$ & $(0 \cdot 013)$ & $(0 \cdot 781)$ \\
\hline Wald $\chi^{2}(11)$ & $127^{* * *}$ & $220 * * *$ & $188^{* * * *}$ & $217 * * *$ & $88 * * *$ \\
\hline Pseudo- $\mathrm{R}^{2}$ & $0 \cdot 041$ & 0.031 & 0.081 & $0 \cdot 057$ & $0 \cdot 013$ \\
\hline $\mathrm{N}$ & 499 & 499 & 499 & 499 & 499 \\
\hline
\end{tabular}

Coefficient estimates from control function models are shown with bootstrapped standard errors clustered at compound level in parentheses. The same control variables as those shown in Tables A3.10 and A3.11 were included in estimation. ${ }^{*} \mathrm{p}<0 \cdot 10,{ }^{* *} \mathrm{p}<0 \cdot 05,{ }^{* * *} \mathrm{p}<0 \cdot 01$. 
Table A3.15: Effects of modern retailers on calorie and nutrient intakes of individuals

from poor households (model results for Figure 3.3)

\begin{tabular}{|c|c|c|c|c|c|}
\hline & $\begin{array}{c}\text { Calorie } \\
\text { (kcal/day) }\end{array}$ & $\begin{array}{l}\text { Protein } \\
\text { (g/day) }\end{array}$ & $\begin{array}{c}\text { Iron } \\
\text { (mg/day) }\end{array}$ & $\begin{array}{c}\text { Zinc } \\
\text { (mg/day) }\end{array}$ & $\begin{array}{c}\text { Vitamin A } \\
(\mu \mathrm{g} \text { retinol/day) }\end{array}$ \\
\hline & $(1)$ & (2) & (3) & (4) & (5) \\
\hline \multicolumn{6}{|l|}{ Adults } \\
\hline Modern retail use & $17 \cdot 070 * *$ & $0.757 * * *$ & $0 \cdot 244 * * *$ & $0 \cdot 045^{*}$ & $3 \cdot 615^{* *}$ \\
\hline (expenditure share, $\%$ ) & $(7 \cdot 656)$ & $(0 \cdot 234)$ & $(0 \cdot 065)$ & $(0 \cdot 025)$ & $(1.470)$ \\
\hline Controls & Yes & Yes & Yes & Yes & Yes \\
\hline \multirow[t]{2}{*}{ First-stage residual } & $-18 \cdot 272 * *$ & $-0.760 * * *$ & $-0 \cdot 260 * * *$ & $-0.059^{*}$ & $-2 \cdot 541$ \\
\hline & $(8 \cdot 971)$ & $(0 \cdot 277)$ & $(0 \cdot 073)$ & $(0 \cdot 034)$ & $(1.668)$ \\
\hline Wald $\chi^{2}(11)$ & 14 & $32 * * *$ & $24 * *$ & $17 * * *$ & $51 * * *$ \\
\hline Pseudo- $\mathrm{R}^{2}$ & $0 \cdot 003$ & $0 \cdot 012$ & $0 \cdot 026$ & $0 \cdot 010$ & 0.012 \\
\hline $\mathrm{N}$ & 226 & 226 & 226 & 226 & 226 \\
\hline \multicolumn{6}{|l|}{ Children } \\
\hline Modern retail use & $22 \cdot 962 * * *$ & $0 \cdot 558 * * *$ & $0 \cdot 118^{* *}$ & $0 \cdot 026$ & $3 \cdot 716^{* * *}$ \\
\hline (expenditure share, \%) & $(5 \cdot 310)$ & $(0 \cdot 199)$ & $(0 \cdot 060)$ & $(0 \cdot 026)$ & $(1.096)$ \\
\hline Controls & Yes & Yes & Yes & Yes & Yes \\
\hline \multirow[t]{2}{*}{ First-stage residual } & $-22 \cdot 306^{* * *}$ & $-0.653^{* * *}$ & $-0 \cdot 147 * *$ & $-0 \cdot 032$ & $-3 \cdot 064 * *$ \\
\hline & $(5 \cdot 857)$ & $(0 \cdot 207)$ & $(0.065)$ & $(0 \cdot 030)$ & $(1 \cdot 250)$ \\
\hline Wald $\chi^{2}(11)$ & $61 * * *$ & $164 * * *$ & $62^{* * * *}$ & $76 * * *$ & $35 * * *$ \\
\hline Pseudo- $\mathrm{R}^{2}$ & $0 \cdot 058$ & 0.039 & $0 \cdot 103$ & $0 \cdot 057$ & 0.016 \\
\hline $\mathrm{N}$ & 175 & 175 & 175 & 175 & 175 \\
\hline
\end{tabular}

Poor households are defined as those with incomes less than $\$ 1.90$ per capita and day. Coefficient estimates from control function models are shown with bootstrapped standard errors clustered at compound level in parentheses. The same control variables as those shown in Tables A3.10 and A3.11 were included in estimation. For Figure 3.3, coefficients and standard errors were multiplied by 10, to show effects of a 10 percentage point increase in the modern retail expenditure share. ${ }^{*} p<0 \cdot 10,{ }^{* * *} p<0 \cdot 05,{ }^{* * *} p<0 \cdot 01$ 
Table A3.16: Effects of modern retailers on calorie and nutrient intakes disaggregated by sex

\begin{tabular}{|c|c|c|c|c|c|}
\hline & $\begin{array}{c}\text { Calorie } \\
\text { (kcal/day) }\end{array}$ & $\begin{array}{c}\text { Protein } \\
(\mathrm{g} / \text { day })\end{array}$ & $\begin{array}{c}\text { Iron } \\
\text { (mg/day) }\end{array}$ & $\begin{array}{c}\text { Zinc } \\
\text { (mg/day) }\end{array}$ & $\begin{array}{c}\text { Vitamin A } \\
(\mu \mathrm{g} / \text { day })\end{array}$ \\
\hline & $(1)$ & (2) & (3) & (4) & (5) \\
\hline \multirow[t]{2}{*}{ Effects on adult males } & $11 \cdot 802 *$ & $0 \cdot 550 * * *$ & $0 \cdot 221^{* *}$ & $0.035^{*}$ & $1 \cdot 556$ \\
\hline & $(6 \cdot 963)$ & $(0 \cdot 201)$ & $(0 \cdot 106)$ & $(0 \cdot 019)$ & $(0 \cdot 972)$ \\
\hline $\mathrm{N}$ & 307 & 307 & 307 & 307 & 307 \\
\hline \multirow[t]{2}{*}{ Effects on adult females } & $13 \cdot 464 * * *$ & $0 \cdot 628 * * *$ & $0 \cdot 140 * * *$ & $0.036^{* * *}$ & $1 \cdot 056$ \\
\hline & $(4 \cdot 498)$ & $(0 \cdot 136)$ & $(0 \cdot 037)$ & $(0 \cdot 012)$ & $(0 \cdot 816)$ \\
\hline $\mathrm{N}$ & 623 & 623 & 623 & 623 & 623 \\
\hline \multirow[t]{2}{*}{ Effects on boys } & $13 \cdot 375^{* *}$ & $0 \cdot 238$ & $0 \cdot 103^{* *}$ & $0 \cdot 018$ & $0 \cdot 571$ \\
\hline & $(6 \cdot 359)$ & $(0 \cdot 163)$ & $(0 \cdot 051)$ & $(0 \cdot 019)$ & $(1 \cdot 336)$ \\
\hline $\mathrm{N}$ & 228 & 228 & 228 & 228 & 228 \\
\hline \multirow[t]{2}{*}{ Effects on girls } & $7 \cdot 399$ & $0.448^{* *}$ & $0 \cdot 138 * * *$ & $0 \cdot 040^{*}$ & 1.964 \\
\hline & $(6 \cdot 419)$ & $(0 \cdot 218)$ & $(0 \cdot 052)$ & $(0 \cdot 023)$ & $(1 \cdot 278)$ \\
\hline $\mathrm{N}$ & 271 & 271 & 271 & 271 & 271 \\
\hline
\end{tabular}

Coefficient estimates from control function models are shown with bootstrapped standard errors clustered at compound level in parentheses. The treatment variable in all models is the share of total food expenditures (in \%) made in modern retailers. The same control variables as those shown in Tables A3.10 and A3.11 were included in estimation. ${ }^{*} \mathrm{p}<0 \cdot 10,{ }^{* *} \mathrm{p}<0 \cdot 05,{ }^{* * *} \mathrm{p}<0 \cdot 01$. 


\title{
4 Supermarkets and affordability of nutritious diets: Evidence from urban
}

\section{Zambia $^{3}$}

\begin{abstract}
Access and affordability of nutritious diets remain formidable challenges in many developing countries, where hunger and micronutrient malnutrition coexist with overweight and obesity. With rapid growth in modern supermarkets replacing/complementing traditional retailers, supermarkets can influence consumer diets and nutrition. Previous research suggests that supermarkets may improve dietary quality. However, none of the available studies analyzed the role of supermarkets on affordability of nutritious diets; largely due to data limitations. Here, we analyze effects of supermarkets on dietary quality and affordability. We use individual-level food-intake data and food price data from Lusaka, Zambia, and control function regression models to account for the likely endogeneity of supermarket food purchases. We find that the cost of a recommended nutritious diet is US\$1.22 per day, of which the largest share is the cost of starchy staples (68\%), followed by fruits (11\%), and meat, eggs, and fish (9\%). However, this diet is not affordable to $41 \%$ of low-income group. Meat, fish, and dairy products are more expensive in supermarkets than in traditional retailers. Nevertheless, buying food in supermarkets increases dietary diversity and intake of nutritious diets, with varying effect sizes among demographic cohorts: men, women, boys, and girls. The positive effects of supermarkets on dietary quality largely come from animal source foods.
\end{abstract}

Keywords: Supermarkets, dietary affordability, dietary diversity, intra-household allocation, Zambia.

JEL classification: O12, Q11, Q12, Q18.

\footnotetext{
3 This paper has been sole-authored by the doctoral student (Makaiko Gonapanyanja Khonje). However, the paper substaintially benefited from useful comments made by Prof. Dr. Matin Qaim and Prof. Stephan Klasen at various stages of the manuscript.
} 


\subsection{Introduction}

Hunger - measured by the prevalence of undernourishment - and micronutrient malnutrition remain widespread public health problems in many developing countries (Ruel et al., 2017; FAO et al., 2019). The rapid growth of modern food retailers, in particular supermarkets and fast-food restaurants, in many developing countries (Reardon et al., 2003; Tschirley et al., 2015; Lu and Reardon, 2018) may affect consumer diets and nutrition. For instance, most urban consumers can access both unhealthy foods and nutritious foods from supermarkets (Asfaw, 2008; Reardon and Timmer, 2014; Rischke et al., 2015; Rupa et al., 2019; Debela et al., 2020). However, recent research suggests that healthy diets - the EAT-Lancet diets - are not affordable to more than 1.58 billion people ( $21 \%$ of the world's population) worldwide, of which $72 \%$ are in South Asia and sub-Saharan Africa (Hirvonen et al., 2020).

Typical for many developing countries, urban consumers in Zambia tend to spend more of their income on food from modern retailers compared to traditional food retailers (Khonje and Qaim, 2019). Hence, with a rapid growth of supermarkets, changes in dietary affordability may significantly affect dietary intake among consumers. Moreover, unaffordability of recommended nutritious foods - such as fruits, vegetables, meat, fish, and dairy products - in many developing countries, may affect nutritional outcomes especially among poor consumers (Colen et al., 2018; Headey et al., 2018; Dizon et al., 2019; Headey et al., 2019; Hirvonen et al., 2020). We therefore seek to address three research questions in this study: First, to what extent are recommended nutritious diets affordable among the urban poor in Africa? Second, do supermarkets contribute to affordability of recommended nutritious diets in low-income countries? And third, what are the effects of supermarkets on dietary quality among demographic cohorts: men, women, boys, and girls in urban Africa?

Empirical studies analyzing the effects of supermarkets on dietary quality and affordability are scarce. Using household-level food consumption data, a few existing studies suggests that supermarket food purchases improve diet quality in developing countries such as Tunisia (Tessier et al., 2008), Vietnam (Rupa et al., 2019), and Kenya (Debela et al., 2020). On cost of nutritious diets, Masters et al. (2018) proposes price indexes that measure the cost of diet diversity and nutrient adequacy in Ghana. They found that the cost of diet diversity index fluctuated seasonally and fruits drove up the cost of nutrient adequacy. Though at global level, other similar studies (e.g., Headey et al., 2018; Headey and Alderman, 2019; Hirvonen et al., 2020) found that healthy diets are not affordable to most people in low-income 
countries. In Ethiopia, Headey et al. (2019) observed that children in proximity to rural markets that sell more non-staple foods have more diverse diets.

While the existing research provides important evidence on the effects of modern retailers such as supermarkets - on dietary quality, and the cost of nutritious diets; several limitations exist. First, none of the previous studies on retail modernization has analyzed effects of supermarkets on the affordability of recommended nutritious diets. Moreover, most previous studies analyzed the cost of nutritious diets only for particular target groups, such as women (e.g., Masters et al., 2018; Alemu et al., 2019; Hirvonen et al., 2020) or children (Headey et al., 2019). Yet, inequalities in dietary affordability could exist among various household members. More importantly, available studies analyzed the cost of nutritious diets using food price data from the World Bank's International Comparison Program, where only standardized food items are included (e.g., Alemu et al., 2019; Hirvonen et al., 2020). Hence, some of country-specific nutritious foods (e.g., local insects, fish, fruits, pulses and some dark green leafy vegetables) are omitted. Finally, none of the available studies on the cost of nutritious diets analyzed the role of modern retailers such as supermarkets.

Using unique data - i.e., recent (2018) food price data and individual-level dietary data collected in urban Zambia, we add to the existing literature in several ways. First, we provide the first empirical study that analyzes effects of using supermarkets on the affordability of recommended nutritious diets in Africa. Second, we also extend the existing literature (e.g., Masters et al., 2018; Hirvonen et al., 2020) on the cost of a nutritious diet in Africa, where it is not yet conclusive and relatively very thin. Third, we expand on limited evidence analyzing effects of supermarkets on dietary quality in Africa, where intra-household food distributions were hardly analyzed. Overall, a better understanding on these issues remains vital in designing cohort-specific policy interventions aimed at tackling micronutrient malnutrition in most developing countries, especially among the urban poor households.

The rest of the article is organized as follows. The next section provides a description on study context, data, and estimation strategy. In the following section, we present and discuss empirical results and the last section concludes. 


\subsection{Data}

\subsubsection{Survey of households and individuals}

The data used in this study were collected through a household survey in Lusaka, the capital city of Zambia, between April and July 2018. Zambia is an ideal setting for this study for two reasons. First, it is one of the southern African countries with rapid growth in modern supermarkets (Tschirley et al., 2015; Ziba and Phiri, 2017). For instance, our own review of internet sources supplemented by key local informant interviews revealed that the number of large shopping malls with supermarkets in Lusaka City increased from one in 1995 to 25 in 2018 (Table 1.1). Moreover, other existing studies (e.g., Khonje and Qaim, 2019) have found that a substantial share $(43 \%)$ of the food consumed by urban households in Lusaka is purchased from modern retailers such as hypermarkets, supermarkets, and fast-food restaurants. Finally, like many other countries in sub-Saharan Africa, Zambia is characterized by a high prevalence of micronutrient malnutrition (Harris et al., 2019; Kaliwile et al., 2019). For instance, among women and children; 98\%, 34-55\%, 26\%, and 19\% are deficient in vitamin $B_{12}$, zinc, vitamin $A$, and iron, respectively (Harris et al., 2019).

We surveyed a total of 475 households from several compounds/sections in Lusaka City using a two-stage random sampling procedure. At the first stage, we purposively selected 14 compounds/sections as primary sampling units based on population distributions, the locations of major shopping malls (see Table 1.1 and Table A4.1 in the Appendix), as well as information from the Lusaka City council on mean income levels in the different compounds. To ensure that the sample is fairly representative of households in the urban parts of Lusaka, we selected compounds with different mean income levels: high, middle, and low. Figure 1.1 shows a spatial distribution of the surveyed compounds/sections and households.

At the second stage, depending on the size of the compound/section, we randomly sampled about 35 households from each compound for study participation. In each sampled household, we interviewed the household head or the adult responsible for food purchase decisions and food preparation. We recruited local enumerators to conduct face-to-face interviews in local languages. The enumerators were trained and supervised by the researchers. The structured questionnaire covered sections on the household demographic structure, economic activities, and income. Food consumption data were collected through a seven-day recall using a list of 140 different food items and capturing quantities, food prices, and sources of each item. Foodintake data were captured at the individual level for up to four randomly selected members of each household: two adults ( $>18$ years) and two children/adolescents (6 months -18 years). 
Individual-level food-intake/dietary data were collected through 24-hour dietary recalls; for small children the recall data were provided by the caregiver. We have complete individuallevel data for 1,429 observations: 295 men, 594 women, 240 boys, and 300 girls (Table A4.1 in the Appendix).

\subsubsection{Measuring dietary affordability and quality}

To measure dietary affordability, we use food price data from food consumption data to calculate the cost of recommended nutritious diets (CoRD). We estimate the CoRD using food price data for 57 food items (Table A4.2 in the Appendix) and the recommended dietary serving rates adapted from the EAT-Lancet (flexitarian) diet (Willett et al., 2019). The serving rates for each food group or item are shown in Table 4.1. We included ten healthy food groups (see Table 4.1) only to calculate the CoRD, following classification by FAO and FHI 360 (2016), Cost of Nutritious Diets Consortium (2018), and Masters et al. (2018).

Table 4.1: Serving rates for a nutritious diet, by food group

\begin{tabular}{|c|c|c|c|}
\hline \multirow{2}{*}{ FG No. } & \multirow{2}{*}{ Food group (FG) } & \multirow{2}{*}{ Food items (examples only) } & \multirow{2}{*}{$\begin{array}{c}\text { Serving rate } \\
\text { (grams/day) }\end{array}$} \\
\hline & & & \\
\hline \multirow[t]{2}{*}{1} & Grains, white roots and tubers & Maize flour, Rice, Bread, Buns, Samosa, Pasta & 232 \\
\hline & & Cassava, Sweet potatoes, Irish potatoes & 50 \\
\hline \multirow[t]{2}{*}{2} & Pulses & Common beans, Green beans/Peas/Pods & 50 \\
\hline & & Soybean & 25 \\
\hline 3 & Nuts and seeds & Groundnut dry/flour & 25 \\
\hline 4 & Dairy & Milk, Cheese, Yoghurt & 250 \\
\hline \multirow[t]{3}{*}{5} & Meat, poultry, and fish & Beef, Goat meat, Pork, Sheep meat & 7 \\
\hline & & Chicken, Ducks & 29 \\
\hline & & Fish & 28 \\
\hline 6 & Eggs & Eggs & 13 \\
\hline 7 & Dark green leafy vegetables & $\begin{array}{l}\text { Bean leaves, Green/Red pepper, Pumpkin leaves, } \\
\text { Rape/Mustard/Chinese }\end{array}$ & 100 \\
\hline 8 & Vitamin A rich vegetables & Cassava leaves, Carrots, Sweet potato leaves, & 100 \\
\hline 9 & Other vegetables & $\begin{array}{l}\text { Cabbage, Cucumber, Egg plants, Frozen } \\
\text { vegetables, Lettuce, Okra, Onions, Tomatoes }\end{array}$ & 100 \\
\hline 10 & All fruits & $\begin{array}{l}\text { Apples, Avocadoes, Bananas, Pineapples, } \\
\text { Oranges/Tangerines, Water melons, Mixed fruits }\end{array}$ & 200 \\
\hline
\end{tabular}

Note: The dietary serving rates are adapted from the EAT-Lancet reference diet (Willett et al., 2019). Full list of food items based on the individual-level food-intake data is shown in Table A4.2 in the Appendix.

In calculating the CoRD, the recommended quantities (Table 4.1) are the same for a specific food group but price (Table A4.2 in the Appendix) varies with each food item in a food group. 
Due to unavailability of local food-based dietary guidelines, we use dietary serving rates from the EAT-Lancet diet, which is a global reference diet for a more plant-based healthy diet. Moreover, other existing studies (e.g., Springmann et al., 2018; Hirvonen et al., 2020) have used the EAT-Lancet diet in low-income countries including Zambia. However, the EATLancet diet ignores difference among regions, age groups, and gender (Sanchez, 2020).

Using the individual-level food-intake/dietary data, we calculated two indicators on dietary quality. First, following classification by Masters et al. (2018), dietary diversity score 1 (DDS1) is calculated by summing the number of healthy food groups consumed in the last 24 hours from the ten food groups: (1) grains, white roots and tubers, (2) pulses, (3) nuts and seeds, (4) dairy, (5) meat, poultry, and fish, (6) eggs, (7) dark green leafy vegetables, (8) vitamin A-rich vegetables, (9) other vegetables, and (10) fruits. DDS1 focuses on intake of nutritious foods shown in Table 4.1. We therefore excluded nutrient-poor foods such as sugary products and beverages in calculating the DDS1. Specific food items in each food group are shown in Table A4.2 in the Appendix. The selected food groups have been linked to nutrient adequacy in several low-income countries (Torheim et al., 2004; Steyn et al., 2006; Arimond et al., 2010).

Finally, to account for other food groups excluded in the DDS1, we also calculated another indicator for dietary quality: the dietary diversity score 2 (DDS2). DDS2 is calculated as a count of the different food groups (out of twelve possible groups) consumed by household members in the last 24 hours (Steyn et al., 2006; Kennedy et al., 2011; FAO and FHI 360, 2016). The twelve food groups and their respective food items used in our scoring are shown in Table A4.2 in the Appendix. Food items are categorized into groups based on their nutritional value. Dietary diversity has been widely used as an indicator of individual-level dietary quality in several developing countries (e.g., Torheim et al., 2004; Steyn et al., 2006; Villa et al., 2011; Headey et al., 2019). Moreover, dietary diversity indicators can be measured quickly using a food list-based method, whereas the quantity of food consumed and its nutrient composition are much more difficult to measure and analyze (Masters et al., 2018). Thus, dietary diversity indicators are unlikely to suffer from some of the measurement errors especially in estimation of nutrient intake (Villa et al., 2011). 


\subsection{Empirical strategy}

\subsubsection{Regression models}

We hypothesize that buying food in supermarkets may influence dietary quality. To test this hypothesis, we estimate regression models of the following form:

$$
D_{i j k}=\beta_{0}+\beta_{1} S M_{i j k}+\rho^{\prime} X_{i j k}+u_{i j k}
$$

where $D_{i j k}$ is the outcome of interest - e.g., DDS1, DDS2, and dietary intake (grams/day) of nutritious foods - for an individual $i$ from household $j$ in compound/section $k . S M_{i j k}$ is our treatment binary variable equal to one if any of the food consumed by household members come from a modern supermarket, and zero otherwise - if all of the foods were obtained from traditional sources (e.g., traditional/wet markets, roadside vendors, grocery stores, neighborhood kiosks or shops, own production). $X_{i j k}$ is a vector of control variables that represents individual and household level characteristics - such as age, education, income, ethnicity (Bemba and Chewa), and religion status. $u_{i j k}$ represents the random error term.

We use Poisson and Tobit estimators for the outcomes that are count and continuous with censored data, respectively. In all models, a positive and significant coefficient for parameter $\left(\beta_{1}\right)$, would show that buying food in supermarkets increases dietary quality: dietary diversity and intake among demographic cohorts. To our knowledge, this hypothesis has not been adequately tested before; largely due to the unavailability of individual-level dietary data.

\subsubsection{Control function approach}

Equation (4.1) can be estimated using standard Poisson/Tobit regression models. However, parameter estimates for supermarket $\left(S M_{i j k}\right)$ may be biased and inconsistent due to several sources of endogeneity. For instance, it is possible that unobservable factors - such as personal taste and preferences for special food products, seasonal discounts, and food safety are omitted in equation (4.1). Further, improved dietary quality could also make individuals more productive in work places, and ultimately this could increase supermarket purchases.

Here, we test and correct for potential endogeneity by using a control function (CF) approach with instrumental variables (Wooldridge, 2010; 2015). In comparison to standard instrumental variable (IV) regressions, the CF estimator is more efficient and can also be used to control for unobserved heterogeneity in non-linear models, such as Poisson models (Wooldridge, 
2010; Rupa et al., 2019). Moreover, the CF approach allows studying the nature of selfselection and estimation of treatment effects for a subpopulation (Wooldridge, 2015).

The $\mathrm{CF}$ approach is estimated as a two-stage regression model procedure. Thus, the first stage regression is estimated using all exogenous variables including IVs, and it is specified as:

$$
S M_{i j k}=\gamma_{0}+\gamma_{1} X_{i j k}+\gamma_{2} Z_{i j k}+\varepsilon_{i j k}
$$

where $Z_{i j k}$ is a vector of instrumental variables identifying outcome equation of interest (i.e., equation (4.1)). $S M_{i j k}$ and $X_{i j k}$ represents supermarket food purchases; expressed as expenditure share, and household living standards as defined in equation (4.1), respectively. $\gamma$ are vectors of the parameters to be estimated, and $\varepsilon_{i j k}$ is an error term.

In the second stage, we rerun equation (4.1) using the residuals from the first stage regression (equation (4.2)), as an additional regressor. Thus, the second-stage model is specified as:

$$
D_{i j k}=\beta_{0}+\beta_{1} S M_{i j k}+\rho^{\prime} X_{i j k}+\rho v_{i j k}+\epsilon_{i j k}
$$

where $D_{i j k}, S M_{i j k}$ and $X_{i j k}$ represents key dependent variables, treatment variable and relevant control variables, respectively, as previous defined in equation (4.1). $v_{i j k}$ is the residual from equation (4.2), and the significance of $\rho$ - i.e., testing $H_{0}: \rho=0-$ is key in testing the exogeneity of the endogenous explanatory variable. If the residual term $\left(v_{i j k}\right)$ is statistically insignificant in equation (4.3), the null hypothesis of no endogeneity bias cannot be rejected, so that the CF approach is not required; in that case, the standard one-stage regression model (equation (4.1)) lead to unbiased and more efficient estimates. However, if the residual term turns out statistically significant, the $\mathrm{CF}$ approach is preferred and controls for endogeneity bias. $\epsilon_{i j k}$ is the random error term. Overall, to account for the heterogeneity among the sampled individuals and the two-stage CF estimation procedure, the standard errors are clustered at city compound or household levels and bootstrapped, respectively.

To meet the exclusion restriction in equation (4.3), we use two instrumental variables. First, we use distance from each household to the nearest supermarket; which is calculated using global positioning system (GPS) data collected during our household survey. A few related studies (e.g., Kimenju et al., 2015; Rischke et al., 2015; Courtemanche et al., 2019; Debela et al., 2020) have used GPS-based distance estimate as an identifying instrument. Second, the number of visits to a shopping mall of the household's closest neighbor in the sample is also 
used as an identifying instrument. Beyond distance and accessibility, we expect that the neighbor's behavior may capture influence through local social networks (Rupa et al., 2019).

To further test the validity of the two instruments, we perform a simple falsification test following Di Falco et al. (2011). A valid instrument should be significantly correlated with treatment variable; supermarket food purchases (i.e., equation (4.2)), but it should not be correlated with the dependent variable (e.g., DDS1 and DDS2) of interest. Regression results are shown in Tables A4.3 and A4.4 in the Appendix. As expected, distance to the closest shopping mall with a supermarket is negatively correlated with supermarket food purchases (Table A4.3). On the other hand, number of visits by the household's closest neighbor to a shopping mall is positively correlated with supermarket food purchases. Moreover, the results suggest that both instruments are relevant and valid as they jointly affect supermarket food purchases (Table A4.3), but not DDS1 and DDS2 directly - i.e., coefficient estimates for the instruments in these models are jointly insignificant (Table A4.4).

\subsection{Results and discussion}

\subsubsection{Cost of recommended nutritious diets}

Table 4.2 present the mean daily cost for recommended nutritious diets; adapted from the EAT-Lancet diet, differentiated by supermarket users, demographic cohorts, and income terciles. We find that on average, the cost of a recommended nutritious diet (CoRD) is estimated to be US\$1.22 per day. As expected, the estimated cost is high for supermarket users (US\$1.24) than non-users (US\$1.18). Interestingly, income terciles comparison show that the average cost is highest (US\$1.25) for low-income consumers than high-income consumers (US\$1.19). The food group whose prices and food quantities contributed the largest share of the total cost is starchy $\operatorname{staples}^{4}(68 \%)$, followed by fruits $(11 \%)$, and meat, eggs, and fish (9\%) (Table 4.2).

Overall, these findings suggest that starchy staples, fruits and animal source foods (ASFs) are relatively expensive in comparison to other nutritious foods such as pulses and nuts in Zambia. This is in line with descriptive results shown in Table A4.2 in the Appendix. Similarly, at global level, Hirvonen et al. (2020) found that ASFs, fruits and vegetables

\footnotetext{
${ }^{4}$ The main starchy staple in Zambia is maize flour, which is sold in most supermarkets as a fortified product. Hence, it is rich in micronutrients such as iron and vitamin A. However, the price of maize flour had increased significantly in 2018 because most parts of Zambia experienced extreme droughts when crops were at critical (e.g., flowering) stage in 2015/2016 and 2017/2018 growing seasons.
} 
Table 4.2: Cost of recommended nutritious diets by supermarket users, demographic cohorts and income terciles

\begin{tabular}{|c|c|c|c|c|c|c|c|c|}
\hline & $\begin{array}{c}\text { Total } \\
\text { (US\$/day) }\end{array}$ & $\begin{array}{c}\text { Starchy staples } \\
\text { (US\$/day) }\end{array}$ & $\begin{array}{c}\text { Meat, eggs and } \\
\text { fish } \\
\text { (US\$/day) }\end{array}$ & $\begin{array}{c}\text { Dairy } \\
\text { (US\$/day) }\end{array}$ & $\begin{array}{l}\text { Pulses and nuts } \\
\text { (US\$/day) }\end{array}$ & $\begin{array}{l}\text { Vegetables } \\
\text { (US\$/day) }\end{array}$ & $\begin{array}{c}\text { Fruits } \\
\text { (US\$/day) }\end{array}$ & $\begin{array}{l}\text { Unaffordability } \\
\text { of nutritious } \\
\text { diets } \\
(\%)\end{array}$ \\
\hline & $(1)$ & $(2)$ & $(3)$ & $(4)$ & (5) & (6) & (7) & (8) \\
\hline \multirow[t]{2}{*}{ Overall $(\mathrm{N}=1,429)$} & $1.22(1.28)$ & $0.83(1.06)$ & $0.11(0.16)$ & $0.06(0.17)$ & $0.02(0.07)$ & $0.07(0.07)$ & $0.13(0.66)$ & $15.40(0.36)$ \\
\hline & $100.00 \%$ & $68.03 \%$ & $9.02 \%$ & $4.92 \%$ & $1.64 \%$ & $5.74 \%$ & $10.66 \%$ & \\
\hline \multicolumn{9}{|l|}{ By supermarket users } \\
\hline Users $(\mathrm{N}=938)$ & $1.24(1.30)$ & $0.86(1.12)$ & $0.11(0.15)$ & $0.07(0.18)$ & $0.02(0.05)$ & $0.07(0.07)$ & $0.12(0.61)$ & $8.53(0.28)$ \\
\hline Non-users $(\mathrm{N}=491)$ & $1.18(1.23)$ & $0.78(0.94)$ & $0.10(0.18)$ & $0.03(0.15)$ & $0.03(0.09)$ & $0.07(0.07)$ & $0.16(0.75)$ & $28.51(0.45)$ \\
\hline \multicolumn{9}{|l|}{ By demographic groups } \\
\hline Men $(\mathrm{N}=295)$ & $1.23(1.52)$ & $0.87(1.39)$ & $0.12(0.21)$ & $0.05(0.15)$ & $0.02(0.05)$ & $0.07(0.07)$ & $0.10(0.59)$ & $10.51(0.31)$ \\
\hline Women $(\mathrm{N}=594)$ & $1.17(1.21)$ & $0.79(0.92)$ & $0.10(0.14)$ & $0.04(0.13)$ & $0.02(0.06)$ & $0.07(0.07)$ & $0.15(0.73)$ & $14.65(0.35)$ \\
\hline Boys $(\mathrm{N}=240)$ & $1.17(1.05)$ & $0.76(0.77)$ & $0.09(0.12)$ & $0.08(0.21)$ & $0.02(0.06)$ & $0.07(0.07)$ & $0.15(0.67)$ & $18.75(0.39)$ \\
\hline Girls $(N=300)$ & $1.33(1.32)$ & $0.93(1.14)$ & $0.12(0.19)$ & $0.08(0.21)$ & $0.02(0.08)$ & $0.06(0.06)$ & $0.12(0.57)$ & $19.00(0.39)$ \\
\hline \multicolumn{9}{|l|}{ By income tercile } \\
\hline Lowest $(\mathrm{N}=506)$ & $1.25(1.49)$ & $0.82(1.21)$ & $0.11(0.22)$ & $0.03(0.14)$ & $0.03(0.09)$ & $0.07(0.07)$ & $0.19(0.82)$ & $41.11(0.49)$ \\
\hline Middle $(\mathrm{N}=472)$ & $1.20(1.05)$ & $0.82(0.85)$ & $0.12(0.15)$ & $0.06(0.17)$ & $0.01(0.05)$ & $0.08(0.07)$ & $0.11(0.59)$ & $2.54(0.16)$ \\
\hline Highest $(\mathrm{N}=451)$ & $1.19(1.24)$ & $0.85(1.09)$ & $0.09(0.09)$ & $0.08(0.19)$ & $0.01(0.05)$ & $0.05(0.06)$ & $0.10(0.50)$ & \\
\hline
\end{tabular}

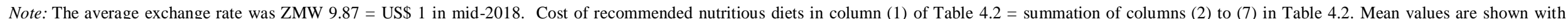

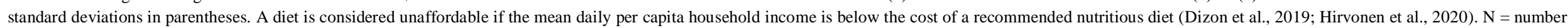
of observations. 
contributed the largest share to the overall cost of the EAT-Lancet diet. This is also consistent with Dizon et al. (2019), where they found that most households overspend on staples and protein foods than vegetables in south Asia: Sri Lanka, Pakistan, Afghanistan, and Bangladesh. Generally, these results are also consistent with other existing studies (e.g., Colen et al., 2018; Headey et al., 2018; Headey and Alderman, 2019; Headey et al., 2019) in lowincome countries.

To further address the first research question, we also report unaffordability of nutritious diets in column (8) of Table 4.2. A diet is considered to be unaffordable if the average CoRD exceeds the mean daily per capita household income (Dizon et al., 2019; Hirvonen et al., 2020). We find that the CoRD is not affordable to $15 \%$ of the sample. However, the prevalence of individuals with total household income per person below the estimated cost of a recommended nutritious diet is highest (41\%) in low-income tercile (Table 4.2). This suggests that some of the nutrient-dense foods - such as meat, eggs, and fish, and fruits - can be relatively expensive to some of the consumers with low income. Hence, it is important for poor consumers to know where to source affordable nutritious foods. This could help to improve poor-quality diets and meet nutrient adequacy among the urban poor in Africa.

\subsubsection{Role of supermarkets on affordability of nutritious diets}

To analyze the role of supermarkets on affordability of nutritious diets (second research question), we re-calculated the CoRD using the recommended dietary serving rates and average food price data shown in Table 4.1 and Table A4.5 in the Appendix, respectively. The results are shown in Figure 4.1. In a simple cost comparison, the results suggest that supermarkets are associated with both positive and negative effects on dietary affordability.

On a positive note, we find that pulses and dark green leafy vegetables are relatively cheaper in supermarkets than in traditional retailers (Figure 4.1). A similar pattern is observed for fruits and eggs, even though the mean differences are statistically insignificant. Minten et al. (2010) observed similar findings in India for vegetables and fruits. This suggests that some nutritious foods are relatively cheaper in supermarkets than in traditional retailers. Therefore, could this be associated with rapid growth of modern retailers such as supermarkets, where a large variety of foods are sold at a lower price? For instance, when asked why a household prefer buying food in supermarkets, almost half (47\%) of them responded that they did so be- 


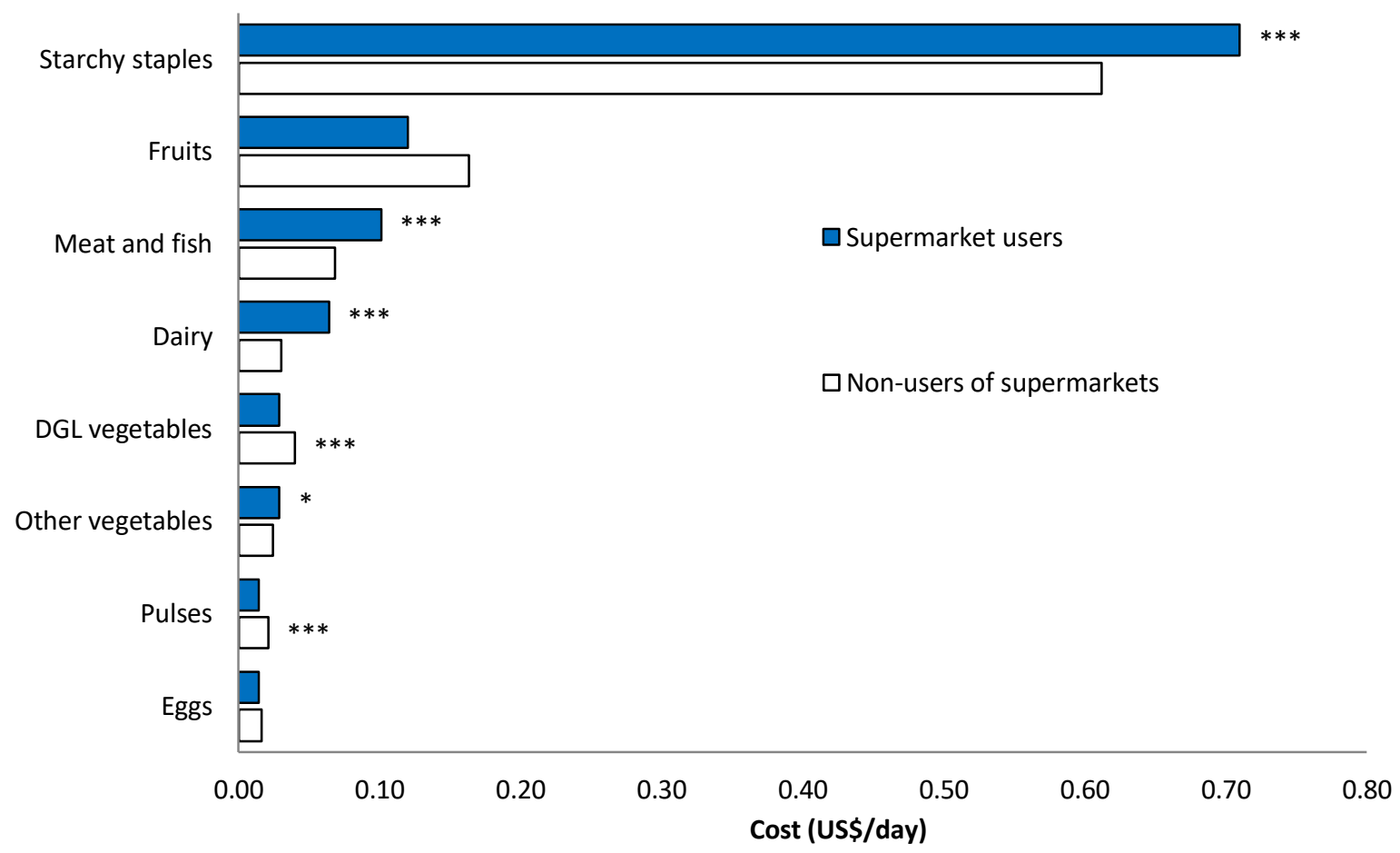

Note: The average exchange rate was ZMW $9.87=$ US\$ 1 in mid-2018. DGL $=$ Dark green leafy vegetables. Detailed summary statistics are shown in Table A4.6 in the Appendix. Asterisk $\left(^{*}\right)$ show significance t-test for the mean differences between users and non-users of supermarkets. $* p<0.1 ; * * p<0.01$.

Figure 4.1: Cost of recommended nutritious diets, by users and non-users of supermarkets

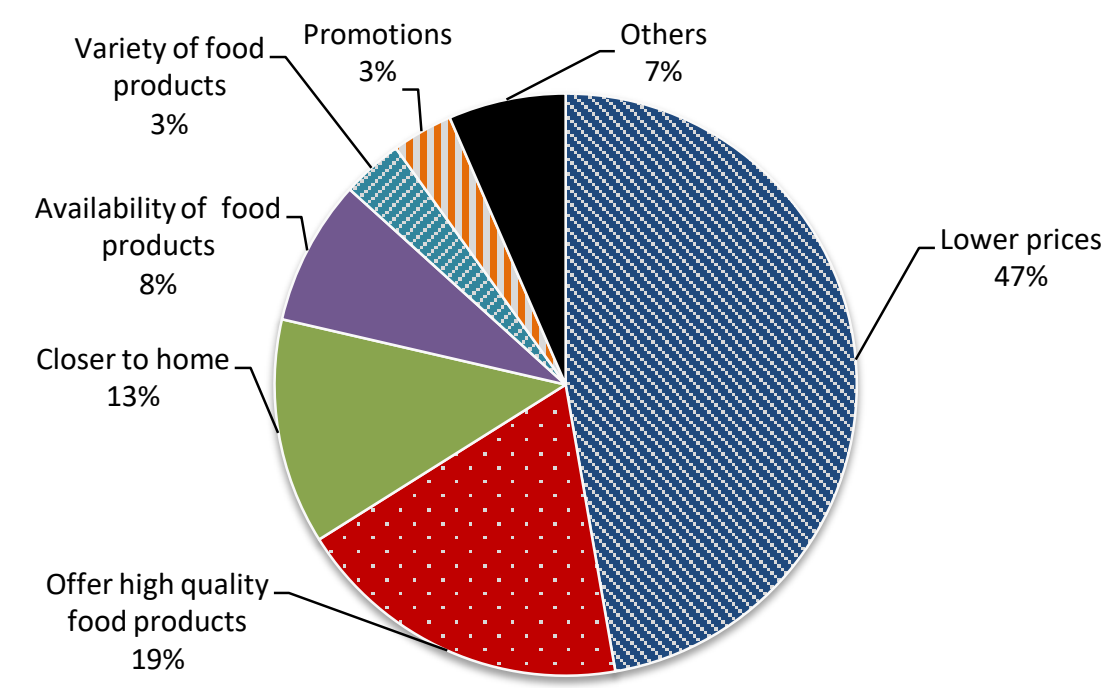

Figure 4.2: Reasons for buying food in modern supermarkets 
-cause of lower prices (Figure 4.2). This is also observed with observational data from urban Kenya (Rischke et al., 2015; Berger and van Helvoirt, 2018).

Moreover, due to higher efficiency and economies-of-scale, modern retailers such as supermarkets may buy farm produce or food products in bulky and sell them at lower prices (Hawkes, 2008; Minten and Reardon, 2008; Lu and Reardon, 2018). Ultimately, traditional shop owners (who have limited resources) may take advantage of lower prices offered by supermarkets especially during promotions or discounts. Hence, traditional shop owners may buy farm produce or food products in bulky for reselling in traditional retail outlets.

On the other hand, we find that starchy staples, animal source foods (ASFs) such as meat, poultry, fish, and dairy products, as well as other vegetables - e.g., cabbage, onions, and tomatoes; full list is shown in Table 4.1 - are more expensive in supermarkets than in traditional retailers (Figure 4.1). This is consistent with findings from other developing countries such as Madagascar (Minten and Reardon, 2008) and Thailand (Schipmann and Qaim, 2011), even though these studies did not analyze dietary costs especially for ASFs. Supermarkets are likely to offer higher quality food products (Schipmann and Qaim, 2011) which are more expensive especially ASFs - than traditional retailers due to better cooling facilities/warehouses (Lu and Reardon, 2018; Khonje and Qaim, 2019). This is also perceived by $19 \%$ of the respondents, who reported high quality food products as an important reason for buying food in supermarkets (Figure 4.2). Moreover, beyond food quality and higher prices, in many developing countries, supermarkets are perceived to fulfill higher food safety standards than traditional retailers (Umberger et al., 2015; Wertheim-Heck et al., 2015).

Nevertheless, the simple cost comparisons in Figure 4.1 does not control for the confounding factors such as income and education. To account for such factors, we run equation (4.3) using costs of nutritious diets as dependent variables. Food-specific regression results are shown in Table A4.7 in the Appendix. Interestingly, we still find that starchy staples, ASFs, and other vegetables are more expensive in supermarkets than in traditional retailers. On the other hand, pulses are relatively cheaper in supermarkets. In summary, these findings suggest that supermarkets have both positive and negative effects on the affordability of nutritious diets. 


\subsubsection{Socioeconomic characteristics}

Table 4.3 present descriptive statistics for key variables used in the regression analyses, differentiated by users and non-users of supermarkets. We generally find that supermarket users have a higher dietary diversity score 2 (DDS2) than non-users. We also find that users of supermarkets have a higher dietary diversity score 1 (DDS1) - measured as count of healthy foods groups consumed in the last 24 hours - than non-users, even though the mean difference is not significant. Further, the results show that men have higher mean values for DDS1 than women and boys (Table A4.8 in the Appendix).

Table 4.3: Summary statistics, by users and non-users of supermarkets

\begin{tabular}{|c|c|c|c|c|c|}
\hline & \multirow{3}{*}{ Units } & \multirow{3}{*}{$\frac{\text { Overall }}{(1)}$} & \multicolumn{2}{|c|}{ Supermarket } & \multirow{2}{*}{$\begin{array}{c}\text { Mean } \\
\text { difference }\end{array}$} \\
\hline & & & Users & Non-users & \\
\hline & & & $(2)$ & (3) & $(4)=(2)-(3)$ \\
\hline \multicolumn{6}{|l|}{ Dependent variables } \\
\hline Dietary diversity score 1 & $\begin{array}{l}\text { Count of healthy food } \\
\text { groups consumed }\end{array}$ & $\begin{array}{c}2.93 \\
(0.93)\end{array}$ & $\begin{array}{c}2.95 \\
(0.92)\end{array}$ & $\begin{array}{c}2.90 \\
(0.95)\end{array}$ & 0.05 \\
\hline Dietary diversity score 2 & $\begin{array}{l}\text { Count of all food } \\
\text { groups consumed }\end{array}$ & $\begin{array}{c}6.57 \\
(1.91)\end{array}$ & $\begin{array}{c}6.74 \\
(1.91)\end{array}$ & $\begin{array}{c}6.24 \\
(1.86)\end{array}$ & $0.49 * * *$ \\
\hline \multicolumn{6}{|l|}{ Selected independent variables } \\
\hline Income & US\$/capita/year & $\begin{array}{c}2504.86 \\
(3221.92)\end{array}$ & $\begin{array}{c}3192.38 \\
(3645.29)\end{array}$ & $\begin{array}{c}1191.45 \\
(1486.26)\end{array}$ & $2000.93 * * *$ \\
\hline Household size & Number of members & $\begin{array}{c}4.72 \\
(1.63)\end{array}$ & $\begin{array}{c}4.51 \\
(1.49)\end{array}$ & $\begin{array}{c}5.12 \\
(1.81)\end{array}$ & $-0.61 * * *$ \\
\hline Age of the respondent & Years & $\begin{array}{c}26.03 \\
(17.27)\end{array}$ & $\begin{array}{c}25.96 \\
(16.38)\end{array}$ & $\begin{array}{c}26.16 \\
(18.87)\end{array}$ & -0.20 \\
\hline Education of the respondent & Schooling years & $\begin{array}{c}8.88 \\
(5.14)\end{array}$ & $\begin{array}{c}9.76 \\
(5.20)\end{array}$ & $\begin{array}{c}7.20 \\
(4.58)\end{array}$ & $2.56 * * *$ \\
\hline Bemba as ethnicity & $1=$ Yes, 0 otherwise & $\begin{array}{c}0.28 \\
(0.45)\end{array}$ & $\begin{array}{c}0.32 \\
(0.47)\end{array}$ & $\begin{array}{c}0.22 \\
(0.42)\end{array}$ & $0.10 * * *$ \\
\hline Protestant as a religion & $1=$ Yes, 0 otherwise & $\begin{array}{c}0.45 \\
(0.50)\end{array}$ & $\begin{array}{c}0.46 \\
(0.50)\end{array}$ & $\begin{array}{c}0.43 \\
(0.50)\end{array}$ & 0.04 \\
\hline $\begin{array}{l}\text { Neighbor's shopping mall } \\
\text { usage }\end{array}$ & $\begin{array}{l}\text { Number of visits per } \\
\text { week }\end{array}$ & $\begin{array}{c}2.59 \\
(2.86)\end{array}$ & $\begin{array}{c}3.10 \\
(3.04)\end{array}$ & $\begin{array}{c}1.61 \\
(2.15)\end{array}$ & $1.49 * * *$ \\
\hline $\begin{array}{l}\text { Household distance to a } \\
\text { supermarket }\end{array}$ & $\begin{array}{l}\text { GPS-measured } \\
\text { distance in } \mathrm{km}\end{array}$ & $\begin{array}{c}2.84 \\
(2.55)\end{array}$ & $\begin{array}{c}2.45 \\
(2.49)\end{array}$ & $\begin{array}{c}3.60 \\
(2.49)\end{array}$ & $-1.16^{* * *}$ \\
\hline Observations & & 1,429 & 938 & 491 & 1,429 \\
\hline
\end{tabular}

Note: The average exchange rate was ZMW $9.87=$ US\$ 1 in mid-2018. Mean values are shown with standard deviations in parentheses. Summary statistics, by age and sex cohorts are shown in Table A4.8 in the Appendix. $* * * p<0.01$.

Furthermore, Table 4.3 results also suggest that several socioeconomic characteristics are different with and without supermarket food purchases. For instance, we find that household income is higher for supermarket users than non-users. We observe a similar pattern for 
education. We also observe that supermarket users have a smaller household size than nonusers. Across demographic cohorts, as expected, men have more education and income than women, boys, and girls (Table A4.8 in the Appendix). Generally, without controlling household living standards such as income and education, the results in Table 4.3 cannot be interpreted as perfect correlations or causal effects.

\subsubsection{Effects of supermarkets on dietary quality}

Table 4.4, column (1) shows Poisson regression (i.e., equation (4.3)) results on the effects of supermarkets on dietary quality: measured as number of healthy food groups consumed in the last 24 hours (third research question). Unlike a previous study in Vietnam (Rupa et al., 2019), after controlling for confounding factors including household income and education, we find that buying food in supermarkets increases intake of nutritious diets by roughly $11 \%$ (panel A of Table 4.4, column (1) $)^{5}$. Interestingly, the results in panel B of Table 4.4 further

Table 4.4: Effects of using supermarkets on dietary diversity

\begin{tabular}{|c|c|c|}
\hline & $\begin{array}{l}\text { Number of healthy food groups } \\
\text { consumed (in last } 24 \text { hours) }\end{array}$ & $\begin{array}{l}\text { Number of all food groups consumed } \\
\text { (in last } 24 \text { hours) }\end{array}$ \\
\hline & $(1)$ & $(2)$ \\
\hline \multicolumn{3}{|c|}{ Panel A: Overall results } \\
\hline \multicolumn{3}{|l|}{ Overall $(N=1,429)$} \\
\hline Supermarket purchase $(1,0)$ & $\begin{array}{c}1.105 * * * \\
(0.017)\end{array}$ & $\begin{array}{c}1.141 * * * \\
(0.017)\end{array}$ \\
\hline \multicolumn{3}{|c|}{ Panel B: Heterogeneous effects, by demographic cohorts } \\
\hline $\operatorname{Men}(N=295)$ & & \\
\hline Supermarket purchase $(1,0)$ & $\begin{array}{c}1.054 * * * \\
(0.014)\end{array}$ & $\begin{array}{c}1.090 * * * \\
(0.014)\end{array}$ \\
\hline \multicolumn{3}{|l|}{ Women $(N=594)$} \\
\hline Supermarket purchase $(1,0)$ & $\begin{array}{c}1.137 * * * \\
(0.014)\end{array}$ & $\begin{array}{c}1.152 * * * \\
(0.016)\end{array}$ \\
\hline \multicolumn{3}{|l|}{ Boys $(N=240)$} \\
\hline Supermarket purchase $(1,0)$ & $\begin{array}{c}1.111 * * * \\
(0.021)\end{array}$ & $\begin{array}{c}1.192 * * * \\
(0.014)\end{array}$ \\
\hline \multicolumn{3}{|l|}{ Girls $(N=300)$} \\
\hline Supermarket purchase $(1,0)$ & $\begin{array}{c}1.096 * * * \\
(0.024)\end{array}$ & $\begin{array}{c}1.132 * * * \\
(0.020)\end{array}$ \\
\hline Controls & Yes & Yes \\
\hline
\end{tabular}

\footnotetext{
${ }^{5}$ Note that coefficient estimates for supermarket in Tables 4.4 and A4.9-A4.11 are calculated as $100 \times$ [incidence-rate ratio (coefficient) - 1]
} 
show that women (14\%) have more diversified diets from supermarket food purchases compared with boys $(11 \%)$, girls $(10 \%)$, and men (5\%). Overall, these findings imply that buying food in supermarkets is associated with increased intake of nutritious diets among adults and children.

While previous studies (e.g., Asfaw 2008; Rischke et al., 2015; Demmler et al., 2018) have found that supermarkets are associated higher consumption of unhealthy diets (negative effects), it is interesting to note that supermarkets may also be associated with higher intake of nutritious diets (positive effects). Nevertheless, results in column (1) of Table 4.4 accounts for intake of nutritious foods only, but not both healthy and less healthy foods.

To analyze the effects of supermarkets on dietary intake of both healthy and less healthy foods, we rerun equation (4.3), focusing on all food groups consumed in the last 24 hours. Thus, we included less healthy foods - such as sugar, salt, and fat-rich foods - in calculating the dietary diversity score 2 (DDS2). Further, DDS2 also includes some of the countryspecific nutritious foods (e.g., local insects - such as caterpillars/ants - and wild mushrooms). Regression results are shown in column (2) of Table 4.4. After controlling for confounding factors, we find that buying food in supermarkets increases dietary diversity by roughly $14 \%$ (panel A of Table 4.4, column (2)). We also find that buying food in supermarkets increases dietary diversity, ranging from $9 \%$ to $19 \%$, for men and boys, respectively (panel B of Table 4.4). This is in line with a recent study in urban Kenya (Debela et al., 2020); even though due to the unavailability of individual-level dietary data - intra-household food distribution could not be analyzed.

As a robustness check, we also rerun equation (4.3) in order to understand the heterogeneous effects of using supermarkets on dietary diversity, by income terciles. About $35 \%$ of the individuals in the sample come from low-income households. Results are shown in Figure 4.3 .

Interestingly, the results show that buying food in supermarkets increases dietary diversity even among low-income consumers by approximately 10-18\% (Figure 4.3). This implies that poor consumers are significantly benefiting from the growth of modern retailers (supermarkets) in urban Zambia. The effect sizes are slightly larger (20-23\%) among highincome consumers as expected (Figure 4.3). These findings suggest that supermarkets have a positive effect on dietary quality even among poor consumers in urban Africa. 


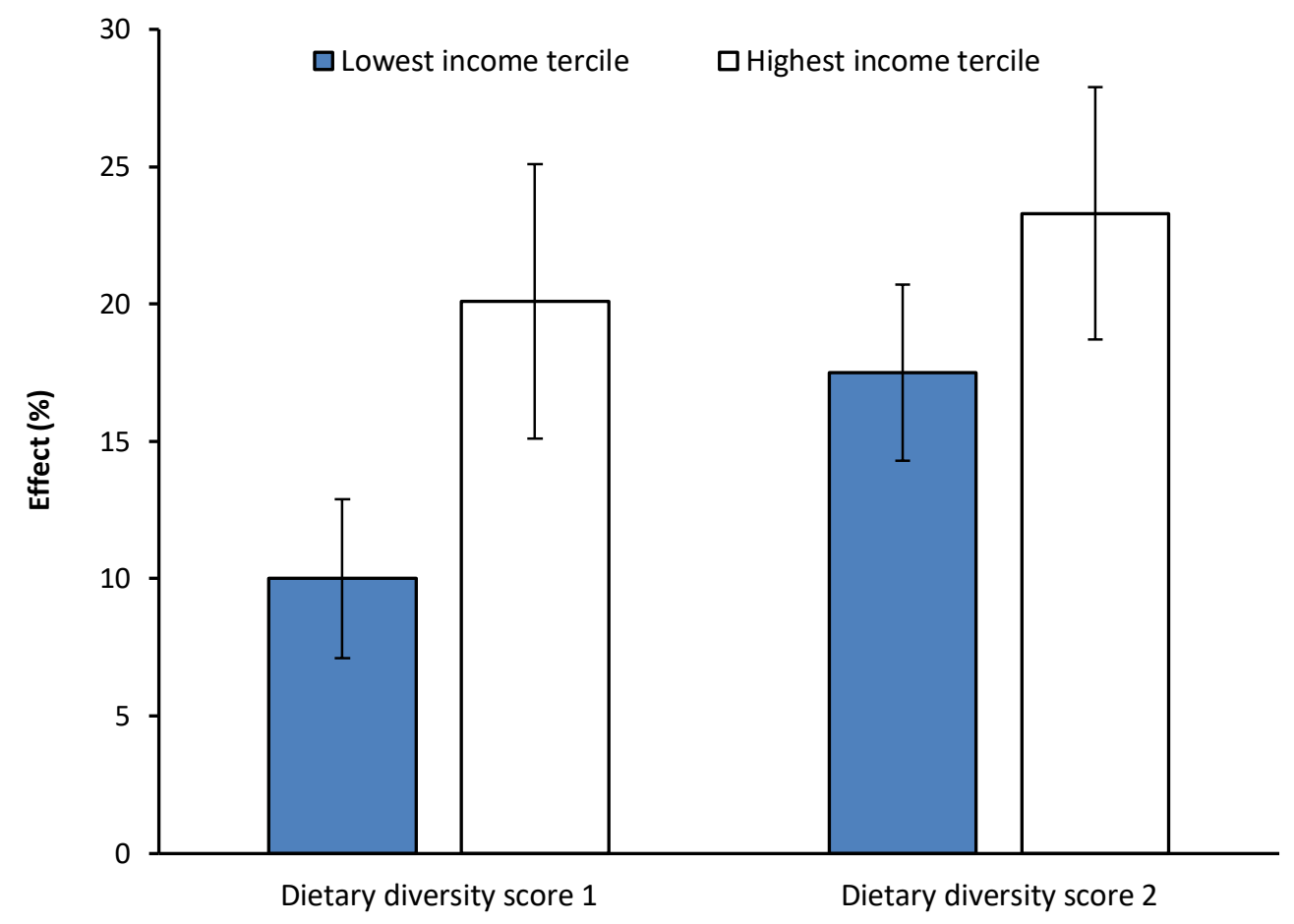

Note: Percentage effects are shown with standard error bars. Use of supermarkets expressed as a dummy variable that takes a value of one if any of the food consumed was purchased in a supermarket and zero if all of the foods consumed were obtained from traditional sources. Effects were estimated with control function models, controlling for income, education, age, and other relevant factors. Full model results are shown in Table A4.11 in the Appendix.

Figure 4.3: Heterogeneous effects of using supermarkets on dietary diversity, by income terciles

\subsubsection{Dietary mechanisms}

To provide some insights on dietary mechanisms, as mentioned before, we conceptualize that buying food in supermarkets may influence dietary quality through higher intake of both healthy and less healthy foods. Figure 4.4 provides a simple comparison on dietary intake with and without supermarket food purchases. The results show that supermarkets have both positive and negative effects on dietary intake. For instance, we find that supermarkets increase the intake of nutrient-dense foods such as meat and dairy products (positive effects).

On the other hand, buying food in supermarkets also increases intake of calorie-dense and nutrient-poor (unhealthy) foods such as sugar and beverages (Figure 4.4). This implies that supermarkets are also likely to be the main source of unhealthy diets. This hypothesis has been confirmed by several studies (e.g., Asfaw 2008; Rischke et al., 2015; Demmler et al., 2018; Khonje and Qaim, 2019) in some developing countries. Nevertheless, Figure 4.4 results are only simple correlations and they do not account for household living standards. 


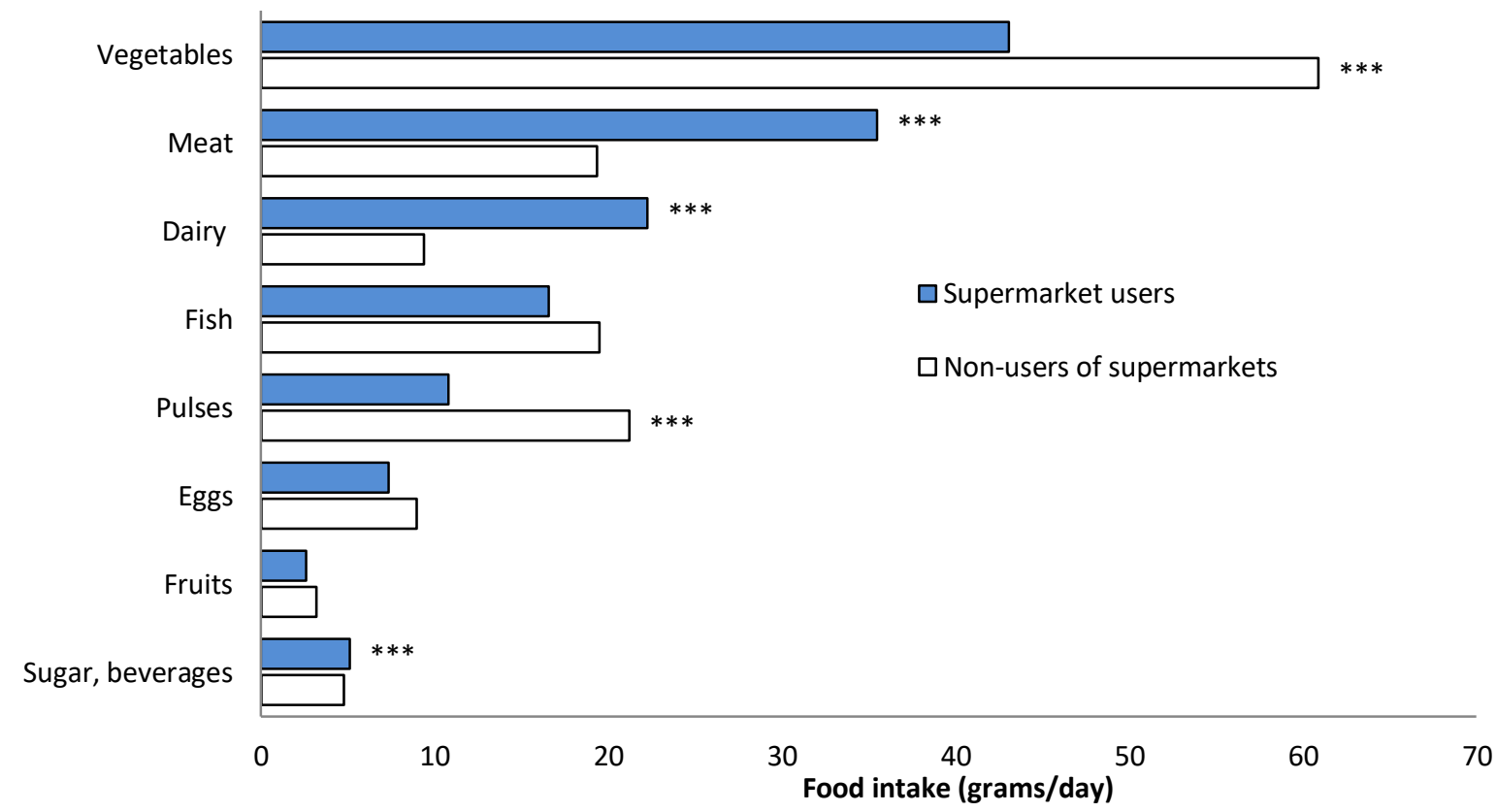

Note: Asterisk (*) show significance t-test for the mean differences between users and non-users of supermarkets. Sugar and beverage values are expressed in natural logarithm. Summary statistics, by age and sex cohorts are shown in Table A4.12 in the Appendix. $* * * p<0.01$.

\section{Figure 4.4: Per capita food intake, by users and non-users of supermarkets}

To control for household living standards, we ran control function (Tobit) regression models (i.e., equation (4.3)) using food intake (grams/day) for different food groups as dependent variables. Food-specific regression results on effects of supermarkets on intensity of dietary intake are shown in Table 4.5. After controlling for confounding factors - such as household

Table 4.5: Effects of using supermarkets on dietary intake

\begin{tabular}{|c|c|c|c|c|c|c|c|}
\hline & \multicolumn{7}{|c|}{ Food intake (grams/day) } \\
\hline & Meat & Dairy & Eggs & Fish & Pulses & Vegetables & Fruits \\
\hline & $(1)$ & $(2)$ & (3) & (4) & $(5)$ & (6) & $(7)$ \\
\hline Supermarket & $1.141 * * *$ & $2.395 * * *$ & $0.834 *$ & $1.785 * * *$ & $-1.528 * * *$ & $0.395 * *$ & 0.799 \\
\hline purchase $(1,0)$ & $(0.158)$ & $(0.840)$ & $(0.498)$ & $(0.286)$ & $(0.394)$ & $(0.156)$ & $(0.933)$ \\
\hline Controls & Yes & Yes & Yes & Yes & Yes & Yes & Yes \\
\hline Wald $\chi^{2}$ & $1021 * * *$ & $156 * * *$ & $74 * * *$ & $178 * * *$ & $313 * * *$ & $308 * * *$ & $33 * * *$ \\
\hline Pseudo- $\mathrm{R}^{2}$ & 0.028 & 0.026 & 0.015 & 0.007 & 0.013 & 0.015 & 0.012 \\
\hline $\mathrm{N}$ & 1,429 & 1,429 & 1,429 & 1,429 & 1,429 & 1,429 & 1,429 \\
\hline
\end{tabular}

Note: We transformed food intake using an inverse hyperbola sine: $\ln \left[\left\{y+\left(y^{2}+1\right)^{0.5}\right\}\right]$, in order to retain zero-valued observations. Coefficient estimates from CF approach through Tobit estimator is shown, with bootstrap standard errors clustered at household level in parentheses. Full model results with all control variables are shown in Table A4.13 in the Appendix. $* p<0.1 ; * * * p<0.01$. 
income and education, we find that buying food in supermarkets significantly increases intake of meat, fish, eggs and dairy products, as well as vegetables.

We also find similar results if the share of total household food expenditures made in supermarkets is used (see Table A4.14 in the Appendix). For instance, we observe that a 10\% increase in the share of supermarket food purchases increases intake of meat, fish, and dairy products by about $4 \mathrm{~g} / \mathrm{day}, 10 \mathrm{~g} / \mathrm{day}$, and $22 \mathrm{~g} /$ day, respectively (Table A4.14). However, we now observe that coefficient estimates for eggs and vegetables are statistically insignificant. Overall, these findings are in line with a recent study in urban Kenya (Debela et al., 2020), where it was also observed that supermarkets are associated with higher consumption of healthy food groups.

In summary, these results confirm the hypothesis that supermarkets have a positive effect on intake of nutritious diets, which largely come from animal source foods (ASFs). The higher dietary intake of ASFs is likely related to better cooling facilities and warehouses in supermarkets compared to traditional retailers (Lu and Reardon, 2018; Khonje and Qaim, 2019). These facilities can improve access to both fresh/perishables and processed versions of ASFs. Conversely, consistent with Figure 4.4 results and an earlier study by Asfaw (2008) in Guatemala, we also find that buying food in supermarkets is negatively associated with lower intake of pulses. This suggests that pulses are largely sourced from traditional food retailers.

The positive effect of supermarkets on dietary intake of ASFs is a welcome finding because ASFs are important source of proteins and micronutrients in many developing countries (Headey et al., 2018; Rupa et al., 2019; Debela et al., 2020; GAIN, 2020). Hence, our results suggest a need to shift food policy from focusing on energy-dense foods to affordable nutritious foods. Modern supermarkets could be one of the platforms to make nutritious foods - i.e., meat, fish, eggs, milk and other dairy products - more affordable especially among lowincome consumers and ultimately reduce health problems related to micronutrient malnutrition. Nevertheless, our findings also suggest that supermarkets lead to higher intake of unhealthy foods such as sugar, salt, and fat-rich foods (Table A4.13 in the Appendix), which might contribute to overweight or obesity especially among adult population. A few existing studies (e.g., Asfaw 2008; Rischke et al., 2015; Demmler et al., 2018; Khonje and Qaim, 2019) found similar results in selected developing countries. 


\subsection{Conclusion}

Access and affordability of nutritious diets remain formidable challenges in many developing countries, where hunger and micronutrient malnutrition coexist with overweight and obesity. Available research suggests that healthy diets are not affordable to most people in subSaharan Africa (Hirvonen et al., 2020). In many developing countries, modern retailers such as supermarkets are spreading rapidly, replacing or complementing traditional food markets and shops as well as grocery stores. This transformation may influence consumer diets and nutrition, through higher consumption of healthy foods as well as less healthy foods. Previous research suggests that supermarkets may improve diet quality (Tessier et al., 2008; Rupa et al., 2019; Debela et al., 2020). However, most existing research analyzed the effects of supermarkets on dietary quality using food consumption data captured at household-level; which hardly accounts for intra-household food distribution. Moreover, the role of supermarkets and other modern retailers on the affordability of nutritious diets was not analyzed in any of the existing studies.

Using food price data and individual-level dietary data from Lusaka, Zambia, and control function regression models to account for the likely endogeneity of supermarket food purchases, we have analyzed the effects of using supermarkets on the affordability of recommended nutritious diet and dietary quality. Our results show that the cost of a recommended nutritious diet is US\$1.22 per day, of which the largest share is the cost of starchy staples (68\%), followed by fruits (11\%), and meat, eggs, and fish (9\%). This is consistent with other existing studies in low-income countries (Headey et al., 2018; Dizon et al., 2019; Headey et al., 2019; Hirvonen et al., 2020). However, on average, this diet is not affordable to $41 \%$ of low-income group. Furthermore, we find that nutrient-dense foods such as meat, fish, and dairy products are more expensive in supermarkets than in traditional retailers; largely due to higher quality food products and safety standards.

Nevertheless, we also find that dietary diversity is higher for consumers using supermarkets than non-users. After controlling for confounding factors such as income and education, we find that buying food in supermarkets increases dietary diversity and intake of nutritious diets, with varying effect sizes among demographic cohorts. The positive effect of supermarkets on dietary quality is channeled through higher intake of animal source foods such as meat, eggs, fish, and dairy products. This is a welcome finding as it may help to reduce micronutrient 
malnutrition. Animal source foods are important source of proteins and micronutrients in many developing countries (Headey et al., 2018; Rupa et al., 2019; Debela et al., 2020).

Our results suggest a need to shift food policy from focusing on energy-dense foods to affordable nutritious foods. Modern retailers - such as supermarkets - could be one of the platforms to make nutritious foods - i.e., meat, fish, eggs, milk, and other dairy products more affordable especially among poor households. Lower prices could come from improvements in local production, higher efficiency in procurements, marketing and trade, and infrastructure developments especially cooling facilities and warehouses. This would ultimately help individuals to access sufficient quantities of nutritious foods; consequently reducing health problems related to micronutrient malnutrition. Nevertheless, our findings also suggest that supermarkets lead to higher intake of less healthy foods, which is in line with a few existing studies (e.g., Asfaw 2008; Rischke et al., 2015; Demmler et al., 2018; Khonje and Qaim, 2019) in some developing countries. Hence, regulatory policies such as regulation of advertisement and promotional campaigns for unhealthy foods and taxes on highlyprocessed foods and beverages with high contents of fats, added sugars and salts (Hawkes et al., 2020) among others, would be ideal policy interventions.

This study has some limitations that could be addressed in future studies. First, we captured individual-level food-intake/dietary data only at one point in time. Hence, we may have systematically missed certain food items that are not consumed on a daily basis. Though more costly, conducting multiple 24-hour dietary recalls could account for the missed food items. Second, our results are specific for Lusaka in Zambia and should not be generalized. The changing food environment may have different effects on dietary quality and affordability in different geographical and socioeconomic contexts. However, it is more likely that supermarkets will have similar effects on dietary quality and affordability in other parts of Africa; which are characterized by a rapid modernization of food environment and a high prevalence of different forms of malnutrition concurrently ( $\mathrm{Lu}$ and Reardon, 2018; FAO et al., 2019). Finally, we used cross-sectional data in this study, which limits the strength of the identification strategy and the options to analyze dynamic effects. 
Appendix A4

Table A4.1: The distribution of the sampled individuals in Lusaka City, Zambia

\begin{tabular}{|c|c|c|c|c|c|c|}
\hline \multirow{2}{*}{$\begin{array}{l}\text { Name of the surveyed } \\
\text { compounds/sections }\end{array}$} & \multirow{2}{*}{$\begin{array}{l}\text { Income } \\
\text { level }\end{array}$} & \multicolumn{4}{|c|}{ By demographic cohort } & \multirow[b]{2}{*}{ All } \\
\hline & & $\begin{array}{c}\text { Men } \\
(>18 \text { years })\end{array}$ & $\begin{array}{c}\text { Women } \\
(>18 \text { years })\end{array}$ & $\begin{array}{c}\text { Boys } \\
(\leq 18 \text { years })\end{array}$ & $\begin{array}{c}\text { Girls } \\
(\leq 18 \text { years })\end{array}$ & \\
\hline $\begin{array}{l}\text { Four: Avondole, Chalala, Kabulonga, } \\
\text { and Woodlands }\end{array}$ & High & 75 & 114 & 40 & 53 & 282 \\
\hline $\begin{array}{l}\text { Four: Chelston, Chilenje, Kabwata, } \\
\text { and PHI }\end{array}$ & Middle & 114 & 239 & 101 & 105 & 559 \\
\hline $\begin{array}{l}\text { Six: Chawama, Chazanga, Gardens, } \\
\text { Kalingalinga, Kaunda Square, and } \\
\text { Ng'ombe }\end{array}$ & Low & 106 & 241 & 99 & 142 & 588 \\
\hline All & & 295 & 594 & 240 & 300 & 1,429 \\
\hline
\end{tabular}


Table A4.2: Descriptive statistics for food prices per gram in Lusaka City, Zambia (mid2018)

\begin{tabular}{|c|c|c|c|c|c|c|c|}
\hline \multirow{2}{*}{ FG No. } & \multirow{2}{*}{ Food group (FG) } & \multirow{2}{*}{ No. } & \multirow{2}{*}{ Food item } & \multicolumn{4}{|c|}{ Food price (ZMW/gram) } \\
\hline & & & & Mean & $\mathrm{SD}$ & Min & Max \\
\hline \multirow[t]{17}{*}{1} & Grains, white roots and tubers & 1 & Maize flour (Mgaiwa) & 0.011 & 0.000 & 0.011 & 0.011 \\
\hline & & 2 & Maize flour (Ufa oyera) & 0.004 & 0.002 & 0.001 & 0.011 \\
\hline & & 3 & Maize green & 0.167 & 0.037 & 0.100 & 0.250 \\
\hline & & 4 & Rice & 0.015 & 0.005 & 0.003 & 0.050 \\
\hline & & 5 & Cassava & 0.003 & 0.000 & 0.003 & 0.003 \\
\hline & & 6 & Pumpkins & 0.033 & 0.007 & 0.010 & 0.050 \\
\hline & & 7 & Irish potatoes & 0.008 & 0.002 & 0.003 & 0.009 \\
\hline & & 8 & Sweet potatoes (SP) & 0.008 & 0.002 & 0.000 & 0.009 \\
\hline & & 9 & Orange fleshed (SP) & 0.009 & 0.000 & 0.009 & 0.009 \\
\hline & & 10 & Porridge & 0.013 & 0.002 & 0.010 & 0.025 \\
\hline & & 11 & Samosa & 0.025 & 0.000 & 0.025 & 0.025 \\
\hline & & 12 & Instant noodles & 0.021 & 0.009 & 0.005 & 0.054 \\
\hline & & 13 & Bread (White) & 0.015 & 0.002 & 0.008 & 0.024 \\
\hline & & 14 & Bread (Brown) & 0.015 & 0.001 & 0.010 & 0.020 \\
\hline & & 15 & Pasta (Spaghetti) & 0.122 & 0.088 & 0.007 & 0.300 \\
\hline & & 16 & Buns & 0.045 & 0.044 & 0.000 & 0.400 \\
\hline & & 17 & Cerelac & 0.025 & 0.012 & 0.001 & 0.046 \\
\hline \multirow[t]{2}{*}{2} & Pulses & 18 & Common beans & 0.015 & 0.003 & 0.004 & 0.016 \\
\hline & & 19 & Soybean & 0.039 & 0.037 & 0.000 & 0.090 \\
\hline \multirow[t]{2}{*}{3} & Nuts and seeds & 20 & Groundnut dry/flour & 0.030 & 0.003 & 0.024 & 0.040 \\
\hline & & 21 & Green beans/Peas/Pods & 0.008 & 0.000 & 0.008 & 0.008 \\
\hline \multirow[t]{3}{*}{4} & Dairy & 22 & Milk & 0.016 & 0.005 & 0.006 & 0.064 \\
\hline & & 23 & Cheese & 0.018 & . & 0.018 & 0.018 \\
\hline & & 24 & Yoghurt & 0.018 & 0.001 & 0.014 & 0.018 \\
\hline \multirow[t]{10}{*}{5} & Meat, poultry, and fish & 25 & Beef & 0.064 & 0.107 & 0.028 & 0.840 \\
\hline & & 26 & Chicken & 0.034 & 0.008 & 0.000 & 0.040 \\
\hline & & 27 & Ducks & 0.059 & 0.002 & 0.055 & 0.060 \\
\hline & & 28 & Sausage-Beef & 0.076 & 0.180 & 0.016 & 1.000 \\
\hline & & 29 & Sausage-Chicken & 0.070 & 0.069 & 0.025 & 0.250 \\
\hline & & 30 & Sausage-Pork & 0.042 & 0.004 & 0.036 & 0.050 \\
\hline & & 31 & Goat meat & 0.027 & 0.002 & 0.025 & 0.030 \\
\hline & & 32 & Pig meat & 0.033 & 0.009 & 0.020 & 0.045 \\
\hline & & 33 & Sheep meat & 0.050 & 0.000 & 0.050 & 0.050 \\
\hline & & 34 & Fish & 0.048 & 0.079 & 0.000 & 0.750 \\
\hline 6 & Eggs & 35 & Eggs & 0.077 & 0.015 & 0.008 & 0.080 \\
\hline \multirow[t]{4}{*}{7} & Dark green leafy vegetables & 36 & Bean leaves & 0.006 & 0.000 & 0.006 & 0.006 \\
\hline & & 37 & Green/Red pepper & 0.007 & 0.000 & 0.007 & 0.007 \\
\hline & & 38 & Pumpkin leaves & 0.006 & 0.000 & 0.006 & 0.006 \\
\hline & & 39 & Rape/Mustard/Chinese & 0.007 & 0.001 & 0.001 & 0.007 \\
\hline \multirow[t]{3}{*}{8} & Vitamin A rich vegetables & 40 & Cassava leaves & 0.007 & 0.000 & 0.007 & 0.007 \\
\hline & & 41 & Carrots & 0.007 & 0.000 & 0.007 & 0.007 \\
\hline & & 42 & Sweet potato leaves & 0.006 & 0.000 & 0.006 & 0.006 \\
\hline \multirow[t]{8}{*}{9} & Other vegetables & 43 & Cabbage & 0.010 & 0.002 & 0.003 & 0.011 \\
\hline & & 44 & Cucumber & 0.004 & 0.002 & 0.003 & 0.007 \\
\hline & & 45 & Egg plants & 0.008 & 0.004 & 0.003 & 0.033 \\
\hline & & 46 & Frozen vegetables & 0.047 & . & 0.047 & 0.047 \\
\hline & & 47 & Lettuce & 0.011 & 0.000 & 0.011 & 0.011 \\
\hline & & 48 & Okra & 0.008 & 0.001 & 0.004 & 0.008 \\
\hline & & 49 & Onions & 0.006 & 0.001 & 0.001 & 0.006 \\
\hline & & 50 & Tomatoes & 0.008 & 0.001 & 0.001 & 0.008 \\
\hline \multirow[t]{3}{*}{10} & Fruits & 51 & Apples & 0.047 & 0.064 & 0.010 & 0.200 \\
\hline & & 52 & Avocadoes & 0.162 & 0.075 & 0.000 & 0.200 \\
\hline & & 53 & Bananas (Ripe) & 0.072 & 0.011 & 0.020 & 0.075 \\
\hline
\end{tabular}




\begin{tabular}{|c|c|c|c|c|c|c|c|}
\hline & & 54 & Pineapples & 0.009 & 0.000 & 0.009 & 0.009 \\
\hline & & 55 & Oranges/Tangerines & 0.047 & 0.000 & 0.047 & 0.047 \\
\hline & & 56 & Water melons & 0.047 & . & 0.047 & 0.047 \\
\hline & & 57 & Mixed fruits & 0.020 & . & 0.020 & 0.020 \\
\hline \multirow[t]{13}{*}{11} & Sugar, salt and fat-rich foods & 58 & Cooking oil/fats & 0.014 & 0.001 & 0.008 & 0.014 \\
\hline & & 59 & Sandwich/Burger & 0.080 & 0.000 & 0.080 & 0.080 \\
\hline & & 60 & Mandazi/Scones & 0.014 & 0.000 & 0.014 & 0.014 \\
\hline & & 61 & Margarine/Butter & 0.032 & 0.006 & 0.025 & 0.045 \\
\hline & & 62 & Jam & 0.030 & 0.000 & 0.030 & 0.030 \\
\hline & & 63 & Pizza (Fresh/Frozen) & 0.134 & 0.021 & 0.030 & 0.138 \\
\hline & & 64 & Tea/Coffee & 0.011 & 0.001 & 0.000 & 0.011 \\
\hline & & 65 & Sugar & 0.017 & 0.005 & 0.005 & 0.027 \\
\hline & & 66 & Biscuits/Cookies & 0.090 & 0.000 & 0.090 & 0.090 \\
\hline & & 67 & Chocolate & 0.250 & 0.212 & 0.100 & 0.400 \\
\hline & & 68 & Ice cream & 0.100 & 0.000 & 0.100 & 0.100 \\
\hline & & 69 & Soft drinks (Coca-cola,..etc) & 0.016 & 0.000 & 0.016 & 0.017 \\
\hline & & 70 & Juice & 0.020 & 0.009 & 0.008 & 0.064 \\
\hline \multirow[t]{2}{*}{12} & Miscellaneous/ & 71 & Mushroom & 0.020 & . & 0.020 & 0.020 \\
\hline & Traditional foods & 72 & Caterpillars/Ants & 0.097 & 0.099 & 0.042 & 0.300 \\
\hline
\end{tabular}

Note: The average exchange rate was ZMW $9.87=$ US\$ 1 in mid-2018. SD = standard deviation. Food prices were collected through our household survey: food consumption data through a seven-day recall. We also validated food prices through our own random price survey during the study supplemented by review of internet sources. 
Table A4.3: First stage regression results on supermarket food purchases (GLM estimates)

\begin{tabular}{|c|c|c|c|c|c|}
\hline & \multicolumn{5}{|c|}{ Share of supermarket purchases $(\%)$} \\
\hline & Overall & Men & Women & Boys & Girls \\
\hline & (1) & (2) & (3) & (4) & (5) \\
\hline \multirow[t]{2}{*}{ Male (dummy) } & 1.750 & & & & \\
\hline & $(1.546)$ & & & & \\
\hline \multirow[t]{2}{*}{ Age of respondent (years) } & $-0.909 * * *$ & 0.108 & -0.323 & 1.195 & -0.657 \\
\hline & $(0.200)$ & $(0.655)$ & $(0.391)$ & $(1.733)$ & $(1.345)$ \\
\hline \multirow[t]{2}{*}{ Age-squared (years) } & $0.009 * * *$ & -0.001 & 0.003 & -0.099 & 0.022 \\
\hline & $(0.003)$ & $(0.008)$ & $(0.004)$ & $(0.091)$ & $(0.069)$ \\
\hline \multirow{2}{*}{ Education of respondent (years) } & $1.959 * * *$ & $2.672 * * *$ & $2.605 * * *$ & 1.469 & 0.756 \\
\hline & $(0.235)$ & $(0.642)$ & $(0.352)$ & $(1.379)$ & $(1.220)$ \\
\hline \multirow{2}{*}{ Household size (individuals) } & $-3.043 * * *$ & -1.742 & $-2.915 * * *$ & $-2.490 * *$ & $-3.198 * * *$ \\
\hline & $(0.466)$ & $(1.068)$ & $(0.684)$ & $(1.264)$ & $(1.045)$ \\
\hline \multirow[t]{2}{*}{ Income (log) } & $6.759 * * *$ & $10.350 * * *$ & $8.275^{* * *}$ & $5.514 * * *$ & $5.088 * * *$ \\
\hline & $(0.519)$ & $(1.651)$ & $(0.998)$ & $(0.957)$ & $(0.908)$ \\
\hline \multirow[t]{2}{*}{ Chewa (dummy) } & 0.086 & 5.953 & $6.087^{*}$ & -2.578 & $-8.003 *$ \\
\hline & $(2.185)$ & $(4.741)$ & (3.499) & $(5.313)$ & $(4.722)$ \\
\hline \multirow[t]{2}{*}{ Tonga (dummy) } & -3.086 & -2.183 & $-6.157 * *$ & 3.695 & -3.691 \\
\hline & $(1.927)$ & $(4.410)$ & $(2.740)$ & $(5.183)$ & $(4.437)$ \\
\hline \multirow[t]{2}{*}{ Catholic (dummy) } & $-4.551 * * *$ & -0.611 & -3.986 & $-11.387 * *$ & -5.039 \\
\hline & $(1.704)$ & $(3.806)$ & $(2.496)$ & $(4.575)$ & $(3.837)$ \\
\hline \multicolumn{6}{|l|}{ Instruments } \\
\hline \multirow{2}{*}{ Neighbor's shopping mall usage (visits/week) } & $2.965 * * *$ & $3.169 * * *$ & $2.784 * * *$ & $2.947 * * *$ & $3.011 * * *$ \\
\hline & $(0.266)$ & $(0.594)$ & $(0.410)$ & $(0.701)$ & $(0.545)$ \\
\hline \multirow{2}{*}{ Distance to a supermarket $(\mathrm{km})$} & $-1.635^{* * *}$ & $-1.373^{*}$ & $-1.228 * * *$ & $-2.140^{* * *}$ & $-1.974 * * *$ \\
\hline & $(0.295)$ & $(0.740)$ & $(0.420)$ & $(0.765)$ & $(0.639)$ \\
\hline Joint significance of instruments: $\chi^{2}(2)$ & $186^{* * *}$ & $38 * * *$ & $62 * * *$ & $33 * * *$ & $52 * * *$ \\
\hline \multirow[t]{2}{*}{ Constant } & $-21.160 * * *$ & $-98.451 * * *$ & $-55.893 * * *$ & -10.037 & -2.969 \\
\hline & $(6.332)$ & $(21.502)$ & $(13.530)$ & $(13.603)$ & $(11.837)$ \\
\hline $\mathrm{N}$ & 1,429 & 295 & 594 & 240 & 300 \\
\hline
\end{tabular}


Table A4.4: Falsification test for instrument validity (Poisson estimates)

\begin{tabular}{|c|c|c|}
\hline & $\begin{array}{l}\text { Number of healthy food groups consumed } \\
\text { (DDS1) }\end{array}$ & $\begin{array}{l}\text { Number of all food groups consumed } \\
\text { (DDS2) }\end{array}$ \\
\hline & Poisson & Poisson \\
\hline & (1) & (2) \\
\hline \multirow[t]{2}{*}{ Male (dummy) } & 0.024 & 0.002 \\
\hline & $(0.224)$ & $(0.877)$ \\
\hline \multirow[t]{2}{*}{ Age of respondent (years) } & 0.003 & 0.000 \\
\hline & $(0.379)$ & $(0.877)$ \\
\hline \multirow[t]{2}{*}{ Age-squared (years) } & -0.000 & -0.000 \\
\hline & $(0.505)$ & $(0.861)$ \\
\hline \multirow{2}{*}{ Education of respondent (years) } & 0.003 & -0.001 \\
\hline & $(0.475)$ & $(0.745)$ \\
\hline \multirow{2}{*}{ Household size (individuals) } & $0.016^{* * *}$ & $0.009 *$ \\
\hline & $(0.002)$ & $(0.080)$ \\
\hline \multirow[t]{2}{*}{ Income (log) } & 0.001 & $0.019 * * *$ \\
\hline & $(0.956)$ & $(0.006)$ \\
\hline \multirow[t]{2}{*}{ Chewa (dummy) } & $0.070 * * *$ & $0.065^{* *}$ \\
\hline & $(0.001)$ & $(0.016)$ \\
\hline \multirow[t]{2}{*}{ Tonga (dummy) } & 0.015 & $0.057^{* *}$ \\
\hline & $(0.600)$ & $(0.017)$ \\
\hline \multirow[t]{2}{*}{ Catholic (dummy) } & $0.042 *$ & 0.033 \\
\hline & $(0.078)$ & $(0.152)$ \\
\hline \multicolumn{3}{|l|}{ Instruments } \\
\hline \multirow{2}{*}{$\begin{array}{l}\text { Neighbor's shopping mall usage } \\
\text { (visits/week) }\end{array}$} & 0.001 & 0.004 \\
\hline & $(0.819)$ & $(0.428)$ \\
\hline \multirow{2}{*}{ Distance to a supermarket (km) } & -0.001 & -0.010 \\
\hline & $(0.926)$ & $(0.102)$ \\
\hline \multirow{2}{*}{ Joint significance of instruments: $\chi^{2}(2)$} & 0.110 & 4.570 \\
\hline & $(0.947)$ & $(0.102)$ \\
\hline \multirow[t]{2}{*}{ Constant } & $0.894 * * *$ & $1.630 * * *$ \\
\hline & $(0.000)$ & $(0.000)$ \\
\hline Wald $\chi^{2}$ & $145^{* * * *}$ & $68 * * *$ \\
\hline $\mathrm{N}$ & 1,429 & 1,423 \\
\hline
\end{tabular}

Note: We excluded individuals who consumed more than 16 food groups in column (2). Distance to a supermarket and the number of visits by the household's closest neighbour to a shopping mall are used as identifying instruments for supermarket food purchases. Standard errors are clustered at compound/section level, and $p$-values are shown in parentheses. ${ }^{*} p<0.1 ; * * p<0.05 ; * * *<0.01$. 
Table A4.5: Descriptive statistics for food prices (ZMW/gram), by food groups, users and non-users of supermarkets

\begin{tabular}{|c|c|c|c|c|}
\hline \multirow{3}{*}{ FG No. } & \multirow{3}{*}{ Food group (FG) } & \multirow[b]{2}{*}{ Overall } & \multicolumn{2}{|c|}{ Supermarket } \\
\hline & & & Users & Non-users \\
\hline & & (1) & (2) & (3) \\
\hline \multirow[t]{2}{*}{1} & Grains, white roots and tubers $(\mathrm{N}=1419)$ & 0.015 & 0.015 & 0.014 \\
\hline & & $(0.017)$ & $(0.018)$ & $(0.016)$ \\
\hline \multirow[t]{2}{*}{2} & Pulses (N=242) & 0.019 & 0.018 & 0.020 \\
\hline & & $(0.018)$ & $(0.015)$ & $(0.021)$ \\
\hline \multirow[t]{2}{*}{3} & Nuts and seeds $(\mathrm{N}=22)$ & 0.023 & 0.026 & 0.020 \\
\hline & & $(0.011)$ & $(0.010)$ & $(0.012)$ \\
\hline \multirow[t]{2}{*}{4} & Dairy $(\mathrm{N}=176)$ & 0.016 & 0.016 & 0.016 \\
\hline & & $(0.004)$ & $(0.004)$ & $(0.003)$ \\
\hline \multirow[t]{2}{*}{5} & Meat, poultry, and fish $(\mathrm{N}=1034)$ & 0.050 & 0.051 & 0.047 \\
\hline & & $(0.091)$ & $(0.101)$ & $(0.055)$ \\
\hline \multirow[t]{2}{*}{6} & Eggs $(N=208)$ & 0.077 & 0.076 & 0.077 \\
\hline & & $(0.015)$ & $(0.016)$ & $(0.014)$ \\
\hline \multirow[t]{2}{*}{7} & Dark green leafy vegetables $(\mathrm{N}=701)$ & 0.006 & 0.006 & 0.007 \\
\hline & & $(0.001)$ & $(0.001)$ & $(0.000)$ \\
\hline \multirow[t]{2}{*}{8} & Vitamin A rich vegetables $(\mathrm{N}=32)$ & 0.006 & 0.006 & 0.006 \\
\hline & & $(0.000)$ & $(0.000)$ & $(0.000)$ \\
\hline \multirow[t]{2}{*}{9} & Other vegetables $(\mathrm{N}=417)$ & 0.008 & 0.008 & 0.007 \\
\hline & & $(0.003)$ & $(0.003)$ & $(0.002)$ \\
\hline \multirow[t]{2}{*}{10} & All fruits $(\mathrm{N}=85)$ & 0.107 & 0.099 & 0.120 \\
\hline & & $(0.074)$ & $(0.071)$ & $(0.078)$ \\
\hline
\end{tabular}

Note: The average exchange rate was ZMW $9.87=$ US\$ 1 in mid-2018. $\mathrm{N}=$ number of observations. Mean values are shown with standard deviations in parentheses. 
Table A4.6: Summary statistics on cost of recommended nutritious diets, by users and non-users of supermarkets

\begin{tabular}{|c|c|c|c|c|}
\hline \multirow{3}{*}{ Cost (US\$/day) } & \multirow{2}{*}{ Overall } & \multicolumn{2}{|c|}{ Supermarkets } & \multirow{2}{*}{ Mean difference } \\
\hline & & Users & Non-users & \\
\hline & (1) & (2) & (3) & $(4)=(2)-(3)$ \\
\hline \multirow[t]{2}{*}{ Total cost } & 1.046 & 1.082 & 0.977 & $0.11^{* * *}$ \\
\hline & $(0.618)$ & $(0.578)$ & $(0.683)$ & \\
\hline \multirow[t]{2}{*}{ Starchy staples } & 0.676 & 0.710 & 0.612 & $0.10^{* * *}$ \\
\hline & $(0.224)$ & $(0.221)$ & $(0.215)$ & \\
\hline \multirow[t]{2}{*}{ Pulses } & 0.016 & 0.014 & 0.021 & $-0.01 * * *$ \\
\hline & $(0.036)$ & $(0.033)$ & $(0.041)$ & \\
\hline \multirow[t]{2}{*}{ Nuts and seeds } & 0.001 & 0.001 & 0.001 & 0.00 \\
\hline & $(0.007)$ & $(0.008)$ & $(0.006)$ & \\
\hline \multirow[t]{2}{*}{ Dairy } & 0.052 & 0.064 & 0.030 & $0.03^{* * *}$ \\
\hline & $(0.144)$ & $(0.157)$ & $(0.109)$ & \\
\hline \multirow[t]{2}{*}{ Meat, poultry and fish } & 0.090 & 0.101 & 0.068 & $0.03^{* * *}$ \\
\hline & $(0.080)$ & $(0.081)$ & $(0.075)$ & \\
\hline \multirow[t]{2}{*}{ Eggs } & 0.015 & 0.014 & 0.016 & -0.00 \\
\hline & $(0.035)$ & $(0.035)$ & $(0.037)$ & \\
\hline \multirow[t]{2}{*}{ Dark green leafy vegetables } & 0.033 & 0.029 & 0.040 & $-0.01 * * *$ \\
\hline & $(0.034)$ & $(0.032)$ & $(0.037)$ & \\
\hline \multirow[t]{2}{*}{ Vitamin A rich vegetables } & 0.001 & 0.001 & 0.002 & -0.00 \\
\hline & $(0.009)$ & $(0.008)$ & $(0.010)$ & \\
\hline \multirow[t]{2}{*}{ Other vegetables } & 0.027 & 0.029 & 0.024 & $0.00^{*}$ \\
\hline & $(0.047)$ & $(0.049)$ & $(0.041)$ & \\
\hline \multirow[t]{2}{*}{ All fruits } & 0.135 & 0.120 & 0.163 & -0.04 \\
\hline & $(0.549)$ & $(0.502)$ & $(0.629)$ & \\
\hline $\mathrm{N}$ & 1,429 & 938 & 491 & 1,429 \\
\hline
\end{tabular}

Note: The average exchange rate was ZMW $9.87=$ US\$ 1 in mid-2018. $\mathrm{N}=$ number of observations. Mean values are shown with standard deviations in parentheses. ${ }^{*} p<0.1 ; * * p<0.01$. 
Table A4.7: Effects of using supermarkets on cost of nutritious diets

\begin{tabular}{|c|c|c|c|c|c|c|c|c|c|}
\hline & \multicolumn{9}{|c|}{ Cost of recommended nutritious diets (ZMW/day) } \\
\hline & $\begin{array}{l}\text { Starchy } \\
\text { staples }\end{array}$ & $\begin{array}{c}\text { Meat, } \\
\text { poultry and } \\
\text { fish }\end{array}$ & Dairy & Eggs & Legumes & $\begin{array}{c}\text { Dark green leafy } \\
\text { vegetables }\end{array}$ & $\begin{array}{l}\text { Vitamin A rich } \\
\text { vegetables }\end{array}$ & $\begin{array}{c}\text { Other } \\
\text { vegetables }\end{array}$ & Fruits \\
\hline & $(1)$ & $(2)$ & (3) & $(4)$ & $(5)$ & (6) & $(7)$ & $(8)$ & $(9)$ \\
\hline \multirow{2}{*}{$\begin{array}{l}\text { Supermarket purchase } \\
(1,0)\end{array}$} & $0.176^{* * *}$ & $0.457 * * *$ & $1.000 * * *$ & 0.167 & $-0.327 * * *$ & 0.011 & -0.150 & $0.200 * * *$ & 0.623 \\
\hline & $(0.022)$ & $(0.033)$ & $(0.337)$ & $(0.103)$ & $(0.066)$ & $(0.025)$ & $(0.182)$ & $(0.044)$ & $(0.833)$ \\
\hline \multirow[t]{2}{*}{ Gender } & -0.010 & 0.031 & -0.130 & $-0.313^{* * *}$ & 0.017 & 0.050 & -0.012 & $0.200^{* * * *}$ & 0.039 \\
\hline & $(0.018)$ & $(0.032)$ & $(0.245)$ & $(0.093)$ & $(0.076)$ & $(0.031)$ & $(0.177)$ & $(0.056)$ & $(0.787)$ \\
\hline \multirow[t]{2}{*}{ Age } & -0.002 & $0.009 *$ & $-0.073^{*}$ & -0.015 & -0.011 & $0.014 * * *$ & -0.020 & $0.017^{* *}$ & 0.015 \\
\hline & $(0.002)$ & $(0.005)$ & $(0.038)$ & $(0.015)$ & $(0.011)$ & $(0.004)$ & $(0.026)$ & $(0.008)$ & $(0.079)$ \\
\hline \multirow[t]{2}{*}{ Age-squared } & 0.000 & $-0.000^{*}$ & 0.001 & 0.000 & 0.000 & $-0.000^{* * *}$ & 0.000 & 0.000 & 0.001 \\
\hline & $(0.000)$ & $(0.000)$ & $(0.000)$ & $(0.000)$ & $(0.000)$ & $(0.000)$ & $(0.000)$ & $(0.000)$ & $(0.001)$ \\
\hline \multirow[t]{2}{*}{ Education } & -0.004 & -0.004 & -0.017 & 0.023 & 0.016 & $-0.009 * *$ & $0.060 * *$ & -0.010 & $-0.180^{*}$ \\
\hline & $(0.003)$ & $(0.006)$ & $(0.042)$ & $(0.017)$ & $(0.011)$ & $(0.004)$ & $(0.027)$ & $(0.009)$ & $(0.101)$ \\
\hline \multirow[t]{2}{*}{ Household size } & -0.001 & 0.004 & -0.089 & $0.078 * * *$ & 0.005 & $0.040^{* * * *}$ & $0.040 *$ & -0.004 & 0.198 \\
\hline & $(0.006)$ & $(0.006)$ & $(0.070)$ & $(0.020)$ & $(0.011)$ & $(0.005)$ & $(0.022)$ & $(0.010)$ & $(0.161)$ \\
\hline \multirow[t]{2}{*}{ Income } & $0.019 * * *$ & $0.030 * * *$ & $0.372 * * *$ & 0.033 & $-0.107 * * *$ & $-0.029 * * *$ & 0.005 & $-0.041^{* * *}$ & 0.292 \\
\hline & $(0.004)$ & $(0.007)$ & $(0.088)$ & $(0.023)$ & $(0.015)$ & $(0.006)$ & $(0.039)$ & $(0.012)$ & $(0.351)$ \\
\hline \multirow[t]{2}{*}{ Chewa } & -0.022 & -0.033 & $-0.626^{*}$ & $0.371 * * *$ & $0.240 * * *$ & 0.021 & -0.304 & $0.437 * * *$ & 0.205 \\
\hline & $(0.026)$ & $(0.041)$ & $(0.337)$ & $(0.117)$ & $(0.074)$ & $(0.035)$ & $(1.276)$ & $(0.055)$ & $(0.967)$ \\
\hline \multirow[t]{2}{*}{ Tonga } & 0.027 & $0.112 * * *$ & 0.144 & $0.215^{* *}$ & 0.004 & -0.046 & -0.814 & $0.346^{* * * *}$ & -1.374 \\
\hline & $(0.020)$ & $(0.031)$ & $(0.300)$ & $(0.103)$ & $(0.074)$ & $(0.032)$ & (5.099) & $(0.047)$ & $(0.995)$ \\
\hline \multirow[t]{2}{*}{ Catholic } & 0.007 & $0.063 * *$ & $0.338^{*}$ & $0.291 * * *$ & $-0.187^{* * *}$ & $0.038^{*}$ & -0.182 & $0.195^{* * *}$ & 1.005 \\
\hline & $(0.019)$ & $(0.027)$ & $(0.192)$ & $(0.084)$ & $(0.043)$ & $(0.021)$ & $(0.179)$ & $(0.037)$ & $(0.626)$ \\
\hline \multirow[t]{2}{*}{ First-stage residual } & -0.001 & $-0.003^{* * *}$ & 0.005 & $-0.007 * * *$ & $0.003 * * *$ & $-0.003 * * *$ & $-0.007 * *$ & $-0.001^{*}$ & -0.019 \\
\hline & $(0.000)$ & $(0.000)$ & $(0.004)$ & $(0.002)$ & $(0.001)$ & $(0.001)$ & $(0.003)$ & $(0.001)$ & $(0.013)$ \\
\hline \multirow[t]{2}{*}{ Constant } & $2.291 * * *$ & $-0.185 * *$ & $-6.751 * * *$ & $-2.211 * * *$ & 0.169 & 0.024 & $-2.902 * * *$ & $-0.658 * * *$ & $-15.588^{* * * *}$ \\
\hline & $(0.060)$ & $(0.075)$ & (1.088) & $(0.270)$ & $(0.168)$ & $(0.064)$ & $(0.398)$ & $(0.142)$ & (3.599) \\
\hline Wald $\chi^{2}$ & $188^{* * * *}$ & $573^{* * * *}$ & $147^{* * * *}$ & $76^{* * * *}$ & $315 * * *$ & $228 * * *$ & $61 * * *$ & $173 * * *$ & $35 * * *$ \\
\hline Pseudo- $R^{2}$ & 0.047 & 0.034 & 0.031 & 0.024 & 0.020 & 0.032 & 0.048 & 0.023 & 0.012 \\
\hline $\mathrm{N}$ & 1,429 & 1,429 & 1,429 & 1,429 & 1,429 & 1,429 & 1,429 & 1,429 & 1,429 \\
\hline
\end{tabular}

Note: We transformed costs of recommended nutritious diets using an inverse hyperbola sine: $\ln \left[\left\{y+\left(y^{2}+1\right)^{0.5}\right\}\right]$, in order to retain zero-valued observations. Coefficient estimates from control function (CF) approach

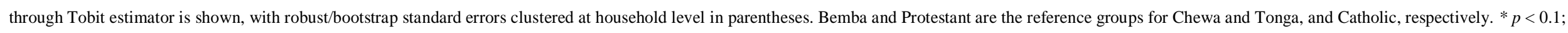
$* * p<0.05$; *** $p<0.01$. 
Table A4.8: Summary statistics, by age and sex cohorts

\begin{tabular}{|c|c|c|c|c|c|}
\hline & \multirow{3}{*}{$\begin{array}{c}\text { Overall } \\
(1)\end{array}$} & \multicolumn{2}{|c|}{ Adults (>18 years ) } & \multicolumn{2}{|c|}{ Children $(\leq 18$ years $)$} \\
\hline & & Men & Women & Boys & Girls \\
\hline & & (2) & (3) & (4) & (5) \\
\hline \multicolumn{6}{|l|}{ Dependent variables } \\
\hline \multirow[t]{2}{*}{ DDS1 } & 2.93 & 3.00 & 2.96 & 2.92 & 2.81 \\
\hline & $(0.93)$ & $(0.88)$ & $(0.92)$ & $(0.93)$ & $(0.98)$ \\
\hline \multirow[t]{2}{*}{ DDS2 } & 6.57 & 6.57 & 6.52 & 6.56 & 6.65 \\
\hline & $(1.91)$ & $(2.01)$ & $(1.91)$ & $(1.71)$ & $(1.95)$ \\
\hline \multicolumn{6}{|l|}{ Selected independent variables } \\
\hline \multirow[t]{2}{*}{ Income } & 2504.86 & 3292.56 & 2524.09 & 2227.02 & 1914.50 \\
\hline & $(3221.92)$ & $(3957.26)$ & $(2913.16)$ & $(3458.60)$ & $(2600.50)$ \\
\hline \multirow[t]{2}{*}{ Household size } & 4.72 & 4.28 & 4.68 & 4.96 & 5.05 \\
\hline & $(1.63)$ & $(1.68)$ & $(1.68)$ & $(1.50)$ & $(1.50)$ \\
\hline \multirow[t]{2}{*}{ Age of the respondent } & 26.03 & 34.79 & 36.65 & 8.75 & 10.21 \\
\hline & $(17.27)$ & $(14.34)$ & $(13.91)$ & $(4.94)$ & $(5.39)$ \\
\hline \multirow[t]{2}{*}{ Education of the respondent } & 8.88 & 12.83 & 10.97 & 3.75 & 4.96 \\
\hline & $(5.14)$ & $(3.00)$ & $(3.61)$ & $(3.96)$ & $(4.29)$ \\
\hline \multirow[t]{2}{*}{ Bemba as ethnicity } & 0.28 & 0.27 & 0.28 & 0.31 & 0.30 \\
\hline & $(0.45)$ & $(0.44)$ & $(0.45)$ & $(0.46)$ & $(0.46)$ \\
\hline \multirow[t]{2}{*}{ Protestant as a religion } & 0.45 & 0.45 & 0.42 & 0.47 & 0.49 \\
\hline & $(0.50)$ & $(0.50)$ & $(0.49)$ & $(0.50)$ & $(0.50)$ \\
\hline \multirow[t]{2}{*}{ Neighbor's shopping mall usage } & 2.59 & 2.78 & 2.53 & 2.47 & 2.62 \\
\hline & $(2.86)$ & $(2.97)$ & $(2.75)$ & $(2.85)$ & $(2.96)$ \\
\hline Household distance to a & 2.84 & 2.57 & 2.95 & 2.95 & 2.81 \\
\hline Shopping mall & $(2.55)$ & $(2.37)$ & $(2.64)$ & $(2.49)$ & $(2.56)$ \\
\hline $\mathrm{N}$ & 1,429 & 295 & 594 & 240 & 300 \\
\hline
\end{tabular}

Note: The average exchange rate was ZMW 9.87 = US\$ 1 in mid-2018. Mean values are shown with standard deviations in parentheses. 
Table A4.9: Effects of using supermarkets on dietary diversity (full model results for panel $A$ in Table 4.4)

\begin{tabular}{|c|c|c|c|c|}
\hline & \multicolumn{2}{|c|}{$\begin{array}{l}\text { Number of healthy food } \\
\text { groups consumed (in last } 24 \text { hours) }\end{array}$} & \multicolumn{2}{|c|}{$\begin{array}{c}\text { Number of all food } \\
\text { groups consumed (in last } 24 \text { hours) }\end{array}$} \\
\hline & Poisson & $C F$ & Poisson & $C F$ \\
\hline & $(1)$ & (2) & (3) & (4) \\
\hline \multirow[t]{2}{*}{ Supermarket purchase $(1,0)$} & 1.030 & $1.105^{* * *}$ & $1.080 * * *$ & $1.141 * * *$ \\
\hline & $(0.029)$ & $(0.017)$ & $(0.027)$ & $(0.017)$ \\
\hline \multirow[t]{2}{*}{ Gender } & 1.025 & $1.025^{*}$ & -0.998 & -0.998 \\
\hline & (0.019) & $(0.015)$ & $(0.017)$ & $(0.011)$ \\
\hline \multirow[t]{2}{*}{ Age } & 1.003 & $1.004 *$ & 1.001 & 1.002 \\
\hline & $(0.002)$ & $(0.002)$ & $(0.002)$ & $(0.002)$ \\
\hline \multirow[t]{2}{*}{ Age-squared } & -1.000 & -1.000 & -1.000 & -1.000 \\
\hline & $(0.000)$ & $(0.000)$ & $(0.000)$ & $(0.000)$ \\
\hline \multirow[t]{2}{*}{ Education } & 1.002 & -1.000 & -0.997 & $-0.996 * *$ \\
\hline & $(0.003)$ & $(0.002)$ & $(0.003)$ & $(0.002)$ \\
\hline \multirow[t]{2}{*}{ Household size } & $1.017 * *$ & $1.019 * * *$ & 1.010 & $1.012 * * *$ \\
\hline & $(0.007)$ & $(0.003)$ & $(0.007)$ & $(0.003)$ \\
\hline \multirow[t]{2}{*}{ Income } & -0.999 & -0.993 & $1.018^{* *}$ & $1.014 * * *$ \\
\hline & $(0.008)$ & $(0.005)$ & $(0.007)$ & $(0.003)$ \\
\hline \multirow[t]{2}{*}{ Chewa } & $1.074 * *$ & $1.080 * * *$ & $1.064 * *$ & $1.068 * * *$ \\
\hline & $(0.036)$ & $(0.021)$ & $(0.030)$ & $(0.015)$ \\
\hline \multirow[t]{2}{*}{ Tonga } & 1.017 & $1.023^{* *}$ & $1.068^{* *}$ & $1.073 * * *$ \\
\hline & $(0.030)$ & $(0.012)$ & $(0.027)$ & $(0.014)$ \\
\hline \multirow[t]{2}{*}{ Catholic } & $1.046^{*}$ & $1.055^{* * *}$ & 1.040 & $1.047 * * *$ \\
\hline & $(0.028)$ & $(0.010)$ & $(0.028)$ & $(0.011)$ \\
\hline \multirow[t]{2}{*}{ First-stage residual } & N/A & $-0.998 * * *$ & N/A & $-0.999 * * *$ \\
\hline & & $(0.0002)$ & & $(0.000)$ \\
\hline \multirow[t]{2}{*}{ Constant } & $2.440 * * *$ & $2.439 * * *$ & $4.868 * * *$ & $4.857 * * *$ \\
\hline & $(0.230)$ & $(0.130)$ & $(0.410)$ & $(0.169)$ \\
\hline Wald $\chi^{2}$ & $22 * *$ & $166^{* * * *}$ & $29^{* *}$ & $223 * * *$ \\
\hline Pseudo- $\mathrm{R}^{2}$ & & 0.004 & & 0.006 \\
\hline $\mathrm{N}$ & 1,429 & 1,429 & 1,429 & 1,429 \\
\hline
\end{tabular}

Note: $\mathrm{N} / \mathrm{A}=$ not applicable. Coefficient estimates from Poisson and control function (CF) approach are shown, with robust/bootstrap standard errors clustered at household level in parentheses. Coefficient etsimates are reported as incidence-rate ratios. Bemba and Protestant are the reference groups for Chewa and Tonga, and Catholic, respectively. We excluded gender in the estimation for cohort-specific results in Table A4.10. * $p<0.1 ; * * p<0.05 ; * * * p<0.01$ 
Table A4.10: Effects of using supermarkets on dietary diversity (full model results for panel B in Table 4.4)

\begin{tabular}{|c|c|c|c|c|c|c|c|c|}
\hline & \multicolumn{4}{|c|}{ Number of healthy food groups consumed (in last 24 hours) } & \multicolumn{4}{|c|}{ Number of all food groups consumed (in last 24 hours) } \\
\hline & Men & Women & Boys & Girls & Men & Women & Boys & Girls \\
\hline & (1) & (2) & (3) & (4) & (5) & (6) & (7) & (8) \\
\hline \multirow{2}{*}{ Supermarket purchase $(1,0)$} & $1.054 * * *$ & $1.137 * * *$ & $1.111^{* * * *}$ & $1.096 * * *$ & $1.090^{* * * *}$ & $1.152 * * *$ & $1.192 * * *$ & $1.132 * * *$ \\
\hline & $(0.014)$ & $(0.014)$ & $(0.021)$ & $(0.024)$ & $(0.014)$ & $(0.016)$ & $(0.014)$ & $(0.020)$ \\
\hline \multirow[t]{2}{*}{ Age } & -0.996 & 1.000 & $1.025^{* *}$ & $1.041^{* * *}$ & -1.000 & 1.004 & $1.030^{* * * *}$ & 1.007 \\
\hline & $(0.003)$ & $(0.003)$ & $(0.008)$ & $(0.009)$ & $(0.002)$ & $(0.003)$ & $(0.004)$ & $(0.007)$ \\
\hline \multirow[t]{2}{*}{ Age-squared } & 1.000 & 1.000 & -0.999 & $-0.999 *$ & 1.000 & -1.000 & $-0.999 *$ & $1.001 * *$ \\
\hline & $(0.000)$ & $(0.000)$ & $(0.000)$ & $(0.000)$ & $(0.000)$ & $(0.000)$ & $(0.000)$ & $(0.000)$ \\
\hline \multirow[t]{2}{*}{ Education } & 1.000 & -0.998 & -0.988 & $-0.979 * * *$ & 1.000 & $-0.994 * *$ & -0.989 & $-0.973 * * *$ \\
\hline & $(0.004)$ & $(0.002)$ & $(0.008)$ & $(0.005)$ & $(0.002)$ & $(0.002)$ & $(0.010)$ & $(0.005)$ \\
\hline \multirow[t]{2}{*}{ Household size } & -1.000 & $1.018^{* * *}$ & $1.008^{* *}$ & $1.041^{* * *}$ & $-0.990 * *$ & $1.010^{* * *}$ & $1.015^{* * *}$ & $1.029 * * *$ \\
\hline & $(0.004)$ & $(0.003)$ & $(0.002)$ & $(0.005)$ & $(0.003)$ & $(0.002)$ & $(0.001)$ & $(0.004)$ \\
\hline \multirow[t]{2}{*}{ Income } & $-0.983 *$ & -0.992 & -0.991 & -0.998 & $1.011^{* *}$ & $1.015^{* * *}$ & $1.015^{* * *}$ & $1.010^{* *}$ \\
\hline & $(0.007)$ & $(0.005)$ & $(0.009)$ & $(0.004)$ & $(0.003)$ & $(0.004)$ & $(0.001)$ & $(0.003)$ \\
\hline \multirow[t]{2}{*}{ Chewa } & 1.027 & 1.018 & $1.210^{* * *}$ & $1.103^{* * *}$ & $1.060^{* * *}$ & $1.035^{*}$ & $1.085^{* * *}$ & $1.090^{* * * *}$ \\
\hline & $(0.026)$ & $(0.018)$ & $(0.015)$ & $(0.024)$ & $(0.018)$ & $(0.017)$ & $(0.009)$ & $(0.018)$ \\
\hline \multirow[t]{2}{*}{ Tonga } & -0.991 & 1.004 & $-0.925^{* * * *}$ & $1.212 * * *$ & $1.073^{* * *}$ & $1.053^{* * * *}$ & $1.037 * * *$ & $1.157 * * *$ \\
\hline & $(0.013)$ & $(0.012)$ & $(0.013)$ & $(0.020)$ & $(0.010)$ & $(0.014)$ & $(0.008)$ & $(0.014)$ \\
\hline \multirow[t]{2}{*}{ Catholic } & $1.057 * * *$ & $1.055^{* * *}$ & $1.035^{*}$ & $1.077 * * *$ & $1.026^{* * *}$ & $1.054 * * *$ & $1.051^{* * *}$ & $1.078^{* * *}$ \\
\hline & $(0.014)$ & $(0.011)$ & $(0.014)$ & $(0.017)$ & $(0.009)$ & $(0.010)$ & $(0.010)$ & $(0.014)$ \\
\hline \multirow[t]{2}{*}{ First-stage residual } & $-0.998^{* * *}$ & $-0.998 * * *$ & $-0.998 * * *$ & $-0.999 *$ & $-0.998 * * *$ & $-0.998 * * *$ & $-0.998 * * *$ & $1.001 * *$ \\
\hline & $(0.0002)$ & $(0.0002)$ & $(0.0002)$ & $(0.0003)$ & $(0.0002)$ & $(0.0002)$ & $(0.0003)$ & $(0.0002)$ \\
\hline \multirow[t]{2}{*}{ Constant } & $3.684 * * *$ & $2.686^{* * * *}$ & $2.489^{* * * *}$ & $1.731^{* * * *}$ & $4.456^{* * * *}$ & $4.610^{* * *}$ & $4.115^{* * *}$ & $4.351^{* * * *}$ \\
\hline & $(0.384)$ & $(0.223)$ & $(0.275)$ & $(0.114)$ & $(0.254)$ & $(0.286)$ & $(0.074)$ & $(0.217)$ \\
\hline Wald $\chi^{2}$ & $133 * * *$ & $312 * * *$ & $1225^{* * * *}$ & $296^{* * * *}$ & $727 * * *$ & $357 * * *$ & $1207 * * *$ & $623 * * *$ \\
\hline Pseudo- $\mathrm{R}^{2}$ & 0.002 & 0.003 & 0.009 & 0.011 & 0.005 & 0.006 & 0.012 & 0.018 \\
\hline $\mathrm{N}$ & 295 & 594 & 240 & 300 & 295 & 594 & 240 & 300 \\
\hline
\end{tabular}

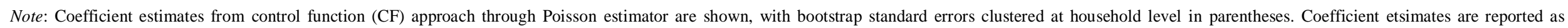
incidence-rate ratios. Bemba and Protestant are the reference groups for Chewa and Tonga, and Catholic, respectively. * $p<0.1 ; * * p<0.05 ; * * * p<0.01$. 
Table A4.11: Heterogeneous effects of using supermarkets on dietary diversity, by income terciles (full model results in Figure 4.3)

\begin{tabular}{|c|c|c|c|c|}
\hline & \multicolumn{2}{|c|}{ Lowest income tercile } & \multicolumn{2}{|c|}{ Highest income tercile } \\
\hline & DDS1 & DDS2 & DDS1 & DDS2 \\
\hline & $(1)$ & $(2)$ & (3) & (4) \\
\hline \multirow[t]{2}{*}{ Supermarket purchase $(1,0)$} & $1.100 * * *$ & $1.175^{* * *}$ & $1.201 * * *$ & $1.233 * * *$ \\
\hline & $(0.029)$ & $(0.032)$ & $(0.050)$ & $(0.046)$ \\
\hline \multirow[t]{2}{*}{ Gender } & $1.063^{* *}$ & -0.994 & 1.006 & 1.009 \\
\hline & $(0.025)$ & $(0.023)$ & $(0.027)$ & $(0.024)$ \\
\hline \multirow[t]{2}{*}{ Age } & 1.004 & 1.002 & 1.007 & 1.006 \\
\hline & $(0.003)$ & $(0.003)$ & $(0.004)$ & $(0.004)$ \\
\hline \multirow[t]{2}{*}{ Age-squared } & -1.000 & -1.000 & -1.000 & -1.000 \\
\hline & $(0.000)$ & $(0.000)$ & $(0.000)$ & $(0.000)$ \\
\hline \multirow[t]{2}{*}{ Education } & 1.002 & -0.998 & -0.997 & $-0.989 * *$ \\
\hline & $(0.004)$ & $(0.004)$ & $(0.004)$ & $(0.004)$ \\
\hline \multirow[t]{2}{*}{ Household size } & $1.027 * * *$ & $1.012 * *$ & $1.018^{*}$ & 1.013 \\
\hline & $(0.005)$ & $(0.005)$ & $(0.009)$ & $(0.007)$ \\
\hline \multirow[t]{2}{*}{ Income } & 1.007 & 1.012 & $-0.922^{* * *}$ & $-0.933^{* * *}$ \\
\hline & $(0.008)$ & $(0.007)$ & $(0.020)$ & $(0.014)$ \\
\hline \multirow[t]{2}{*}{ Chewa } & $1.090^{* *}$ & $1.083^{* * *}$ & $1.118^{*}$ & 1.052 \\
\hline & $(0.029)$ & $(0.026)$ & $(0.050)$ & $(0.029)$ \\
\hline \multirow[t]{2}{*}{ Tonga } & 1.036 & $1.110^{* * *}$ & $1.055^{*}$ & $1.103^{* * *}$ \\
\hline & $(0.027)$ & $(0.032)$ & $(0.025)$ & $(0.022)$ \\
\hline \multirow[t]{2}{*}{ Catholic } & $1.082 * * *$ & $1.092 * * *$ & 1.027 & $1.044 *$ \\
\hline & $(0.019)$ & $(0.022)$ & $(0.024)$ & $(0.019)$ \\
\hline \multirow[t]{2}{*}{ First-stage residual } & -0.999 & $-0.998^{*}$ & $-0.997 * * *$ & $-0.998^{* * *}$ \\
\hline & $(0.001)$ & $(0.001)$ & $(0.000)$ & $(0.000)$ \\
\hline \multirow[t]{2}{*}{ Constant } & $2.009 * * *$ & $4.656^{* * *}$ & $5.090^{* * * *}$ & $11.397 * * *$ \\
\hline & $(0.158)$ & $(0.239)$ & $(1.349)$ & $(2.121)$ \\
\hline Wald $\chi^{2}$ & $105^{* * *}$ & $161^{* * *}$ & $106^{* * *}$ & $175^{* * *}$ \\
\hline Pseudo-R ${ }^{2}$ & 0.007 & 0.011 & 0.007 & 0.010 \\
\hline $\mathrm{N}$ & 506 & 506 & 451 & 451 \\
\hline
\end{tabular}

Note: Coefficient estimates from control function (CF) approach through Poisson estimator are shown, with bootstrap standard errors clustered at household level in parentheses. $* p<0.1 ; * * p<0.05 ; * * *<<0.01$. 
Table A4.12: Per capita food intake, by age and sex cohorts

\begin{tabular}{|c|c|c|c|c|}
\hline \multirow{3}{*}{ Food intake (g/day) } & \multicolumn{2}{|c|}{ Adults (>18 years ) } & \multicolumn{2}{|c|}{ Children $(\leq 18$ years $)$} \\
\hline & Men & Women & Boys & Girls \\
\hline & (1) & (2) & (3) & (4) \\
\hline \multirow[t]{2}{*}{ Meat } & 36.26 & 32.03 & 26.12 & 26.12 \\
\hline & $(47.80)$ & (44.43) & (31.63) & $(31.63)$ \\
\hline \multirow[t]{2}{*}{ Dairy products } & 19.07 & 15.69 & 19.76 & 19.76 \\
\hline & $(76.85)$ & $(67.83)$ & $(55.96)$ & $(55.96)$ \\
\hline \multirow[t]{2}{*}{ Eggs } & 9.52 & 7.98 & 3.98 & 3.98 \\
\hline & $(38.91)$ & (27.16) & $(14.25)$ & $(14.25)$ \\
\hline \multirow[t]{2}{*}{ Fish } & 16.09 & 23.12 & 14.78 & 14.78 \\
\hline & $(35.51)$ & $(67.53)$ & (49.46) & $(49.46)$ \\
\hline \multirow[t]{2}{*}{ Pulses } & 16.15 & 14.88 & 12.03 & 12.03 \\
\hline & $(65.73)$ & $(50.99)$ & $(38.12)$ & $(38.12)$ \\
\hline \multirow[t]{2}{*}{ Vegetables } & 50.81 & 57.27 & 46.37 & 46.37 \\
\hline & $(63.52)$ & $(98.29)$ & $(76.51)$ & (76.51) \\
\hline \multirow[t]{2}{*}{ Fruits } & 2.50 & 3.66 & 2.52 & 2.52 \\
\hline & $(15.37)$ & $(21.91)$ & (13.25) & (13.25) \\
\hline \multirow[t]{2}{*}{ Sugar, beverages } & 168.55 & 157.76 & 115.83 & 115.83 \\
\hline & (196.14) & $(191.50)$ & $(144.02)$ & $(144.02)$ \\
\hline $\mathrm{N}$ & 295 & 594 & 240 & 300 \\
\hline
\end{tabular}


Table A4.13: Effects of using supermarkets on dietary intake (full model results in Table 4.5)

\begin{tabular}{|c|c|c|c|c|c|c|c|c|}
\hline & \multicolumn{8}{|c|}{ Food intake (grams/day) } \\
\hline & Meat & Dairy & Eggs & Fish & Pulses & Vegetables & Fruits & Sugar, Beverages \\
\hline & (1) & (2) & (3) & (4) & $(5)$ & (6) & $(7)$ & $(8)$ \\
\hline \multirow[t]{2}{*}{ Supermarket purchase $(1,0)$} & $1.141^{* * *}$ & $2.395 * * *$ & $0.834 *$ & $1.785 * * *$ & $-1.528 * * *$ & $0.395^{* *}$ & 0.799 & $0.914 * * *$ \\
\hline & $(0.158)$ & $(0.840)$ & $(0.498)$ & $(0.286)$ & $(0.394)$ & $(0.156)$ & $(0.933)$ & $(0.151)$ \\
\hline \multirow[t]{2}{*}{ Gender } & 0.148 & -0.304 & $-1.499 * * *$ & 0.290 & 0.223 & $0.478 * * *$ & 0.145 & $-0.268^{*}$ \\
\hline & $(0.169)$ & $(0.608)$ & $(0.468)$ & $(0.345)$ & $(0.441)$ & $(0.174)$ & $(0.880)$ & $(0.140)$ \\
\hline \multirow[t]{2}{*}{ Age } & 0.002 & $-0.191 * *$ & -0.057 & 0.082 & -0.027 & $0.096 * * *$ & 0.032 & 0.030 \\
\hline & $(0.027)$ & $(0.092)$ & $(0.074)$ & $(0.052)$ & $(0.062)$ & $(0.021)$ & $(0.090)$ & $(0.020)$ \\
\hline \multirow[t]{2}{*}{ Age-squared } & 0.000 & $0.002 *$ & 0.000 & -0.001 & 0.000 & $-0.001 * * *$ & 0.001 & 0.000 \\
\hline & $(0.000)$ & $(0.001)$ & $(0.001)$ & $(0.001)$ & $(0.001)$ & $(0.000)$ & $(0.001)$ & $(0.000)$ \\
\hline \multirow[t]{2}{*}{ Education } & $0.076^{* * * *}$ & -0.026 & 0.105 & -0.094 & 0.057 & $-0.048^{* *}$ & $-0.225^{*}$ & -0.001 \\
\hline & $(0.028)$ & $(0.102)$ & $(0.084)$ & $(0.062)$ & $(0.062)$ & $(0.023)$ & $(0.115)$ & $(0.021)$ \\
\hline \multirow[t]{2}{*}{ Household size } & $0.147 * * *$ & -0.243 & $0.340^{* * *}$ & $-0.270 * * *$ & -0.016 & $0.213 * * *$ & 0.198 & 0.001 \\
\hline & $(0.034)$ & $(0.171)$ & $(0.101)$ & $(0.056)$ & $(0.058)$ & $(0.025)$ & $(0.184)$ & $(0.033)$ \\
\hline \multirow[t]{2}{*}{ Income } & $0.543 * * *$ & $0.925 * * *$ & $0.200 *$ & $-0.364 * * *$ & $-0.650^{* * *}$ & $-0.226 * * *$ & 0.382 & $0.230^{* * * *}$ \\
\hline & $(0.038)$ & $(0.223)$ & $(0.119)$ & $(0.060)$ & $(0.083)$ & $(0.040)$ & $(0.407)$ & $(0.041)$ \\
\hline \multirow[t]{2}{*}{ Chewa } & $-0.370^{* *}$ & $-1.679 * *$ & $1.694 * * *$ & 0.452 & $1.578^{* * *}$ & $0.501 * * *$ & 0.349 & $0.579 * * *$ \\
\hline & $(0.185)$ & $(0.832)$ & $(0.572)$ & $(0.407)$ & $(0.414)$ & $(0.185)$ & (1.091) & $(0.138)$ \\
\hline \multirow[t]{2}{*}{ Tonga } & 0.085 & 0.374 & $1.025 * *$ & $0.837 * *$ & 0.166 & 0.162 & -1.529 & $0.376^{* * *}$ \\
\hline & $(0.138)$ & $(0.744)$ & $(0.507)$ & $(0.354)$ & $(0.436)$ & $(0.154)$ & (1.109) & $(0.130)$ \\
\hline \multirow[t]{2}{*}{ Catholic } & 0.094 & $0.814^{*}$ & $1.522 * * *$ & $0.916^{* * *}$ & $-0.887 * * *$ & $0.687 * * *$ & 0.897 & $0.446 * * *$ \\
\hline & $(0.119)$ & $(0.473)$ & $(0.412)$ & $(0.253)$ & $(0.244)$ & $(0.115)$ & $(0.704)$ & $(0.117)$ \\
\hline \multirow[t]{2}{*}{ First-stage residual } & $0.007 * * *$ & 0.013 & $-0.032 * * *$ & $-0.030^{* * *}$ & $0.018^{* * * *}$ & $-0.018^{* * *}$ & -0.020 & $-0.006^{* *}$ \\
\hline & $(0.002)$ & $(0.010)$ & $(0.009)$ & $(0.005)$ & $(0.006)$ & $(0.003)$ & $(0.015)$ & $(0.002)$ \\
\hline \multirow[t]{2}{*}{ Constant } & $-6.393^{* * * *}$ & $-16.568^{* * *}$ & $-11.355^{* * *}$ & $-1.768 * * *$ & 0.890 & $1.568 * * *$ & $-18.151^{* * *}$ & 0.569 \\
\hline & $(0.429)$ & (2.758) & (1.406) & $(0.652)$ & $(0.935)$ & $(0.396)$ & $(4.255)$ & $(0.441)$ \\
\hline Wald $\chi^{2}$ & $1021 * * *$ & $156^{* * *}$ & $74 * * *$ & $178 * * *$ & $313^{* * * *}$ & $308^{* * * *}$ & $33^{* * *}$ & $244 * * *$ \\
\hline Pseudo- $\mathrm{R}^{2}$ & 0.028 & 0.026 & 0.015 & 0.007 & 0.013 & 0.015 & 0.012 & 0.014 \\
\hline $\mathrm{N}$ & 1,429 & 1,429 & 1,429 & 1,429 & 1,429 & 1,429 & 1,429 & 1,429 \\
\hline
\end{tabular}

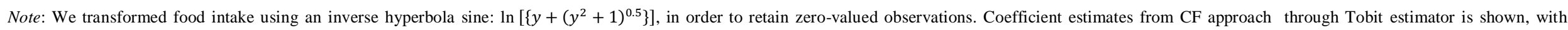

robust/bootstrap standard errors clustered at household level in parentheses. Bemba and Protestant are the reference groups for Chewa and Tonga, and Catholic, respectively. * $p<0.1 ; * * p<0.05 ; * * * p<0.01$. 
Table A4.14: Effects of using supermarkets on dietary intake: Robustness check

\begin{tabular}{|c|c|c|c|c|c|c|c|c|}
\hline & \multicolumn{8}{|c|}{ Food intake (grams/day) } \\
\hline & Meat & Dairy & Eggs & Fish & Pulses & Vegetables & Fruits & Sugar, Beverages \\
\hline & $(1)$ & $(2)$ & (3) & (4) & $(5)$ & (6) & (7) & $(8)$ \\
\hline \multirow{2}{*}{$\begin{array}{l}\text { Share of supermarket } \\
\text { purchases }(\%)\end{array}$} & $0.377 * * *$ & $2.197 * * *$ & 0.282 & $1.010 * * *$ & 0.437 & -0.208 & $-1.623^{* * *}$ & $1.945^{* * *}$ \\
\hline & $(0.101)$ & $(0.729)$ & $(0.329)$ & $(0.184)$ & $(0.284)$ & $(0.182)$ & $(0.548)$ & $(0.408)$ \\
\hline \multirow[t]{2}{*}{ Gender } & 3.475 & -13.388 & $-21.157^{* * *}$ & -2.156 & 3.216 & $9.839 * *$ & 3.590 & $-20.101^{*}$ \\
\hline & (3.216) & (22.368) & $(7.544)$ & (7.719) & (12.536) & $(4.887)$ & (10.804) & $(11.072)$ \\
\hline \multirow[t]{2}{*}{ Age } & 0.451 & $-5.399^{*}$ & 0.116 & $2.881 * *$ & 1.159 & $2.490 * * *$ & -0.627 & $3.707 * * *$ \\
\hline & $(0.534)$ & $(3.065)$ & $(1.347)$ & $(1.261)$ & $(1.443)$ & $(0.964)$ & $(1.236)$ & $(1.376)$ \\
\hline \multirow[t]{2}{*}{ Age-squared } & -0.006 & 0.054 & -0.008 & $-0.028^{*}$ & -0.007 & $-0.026 * *$ & 0.017 & $-0.047^{* * *}$ \\
\hline & $(0.007)$ & $(0.037)$ & $(0.017)$ & $(0.016)$ & $(0.018)$ & $(0.012)$ & $(0.016)$ & $(0.017)$ \\
\hline \multirow[t]{2}{*}{ Education } & $1.373^{* *}$ & -2.084 & 0.727 & $-3.363^{* *}$ & -1.589 & -0.330 & 0.081 & -0.806 \\
\hline & $(0.580)$ & (3.884) & $(1.688)$ & $(1.345)$ & $(1.770)$ & (1.019) & $(1.683)$ & $(1.843)$ \\
\hline \multirow[t]{2}{*}{ Household size } & $2.595^{* * * *}$ & -3.634 & $4.922 * * *$ & $-7.819^{* * *}$ & 0.355 & $2.472 * *$ & -4.661 & $4.901^{*}$ \\
\hline & $(0.651)$ & (6.100) & $(1.869)$ & $(1.361)$ & (1.903) & (1.195) & $(2.877)$ & (2.659) \\
\hline \multirow[t]{2}{*}{ Income } & $6.360 * * *$ & 17.021 & 3.269 & $-14.329^{* * *}$ & $-24.004 * * *$ & $-6.412 * * *$ & $19.781^{* *}$ & $9.317^{* *}$ \\
\hline & $(1.058)$ & $(11.157)$ & (3.373) & $(1.960)$ & (3.095) & $(1.802)$ & $(8.044)$ & $(4.594)$ \\
\hline \multirow[t]{2}{*}{ Chewa } & -3.154 & $-56.362 * *$ & $26.666^{* * *}$ & 7.956 & $58.433 * * *$ & $23.394 * * *$ & 2.167 & $33.414 * * *$ \\
\hline & (3.988) & $(28.276)$ & $(8.741)$ & $(8.837)$ & $(10.049)$ & $(6.301)$ & $(14.918)$ & $(12.681)$ \\
\hline \multirow[t]{2}{*}{ Tonga } & $5.308^{*}$ & 14.488 & $17.262^{*}$ & $19.909 * * *$ & 8.453 & $10.486^{* *}$ & $-30.990 * *$ & $19.187^{*}$ \\
\hline & (2.998) & (26.435) & $(9.943)$ & (7.100) & $(9.787)$ & $(4.392)$ & (13.837) & (11.313) \\
\hline \multirow[t]{2}{*}{ Catholic } & 2.067 & 25.262 & $26.336 * * *$ & $22.412 * * *$ & -1.617 & $31.168 * * *$ & 1.166 & $21.264 * *$ \\
\hline & (3.044) & (22.589) & $(6.625)$ & (6.146) & $(9.736)$ & (3.224) & (9.106) & (8.764) \\
\hline \multirow[t]{2}{*}{ First-stage residual } & -0.041 & -1.085 & $-0.674^{*}$ & $-1.392 * * *$ & $-0.485^{*}$ & -0.208 & $1.479 * * *$ & $-1.612 * * *$ \\
\hline & $(0.098)$ & $(0.817)$ & $(0.364)$ & $(0.190)$ & $(0.269)$ & $(0.191)$ & $(0.558)$ & $(0.421)$ \\
\hline \multirow[t]{2}{*}{ Constant } & $-105.054 * * *$ & $-478.391^{* * *}$ & $-197.901^{* * *}$ & 7.438 & 32.439 & $26.364 * *$ & $-297.588 * * *$ & $-113.513^{* * *}$ \\
\hline & (10.308) & (107.117) & (30.998) & (13.596) & (28.230) & (11.067) & (75.808) & $(36.165)$ \\
\hline Wald $\chi^{2}$ & $987 * * *$ & $108 * * *$ & $53 * * *$ & $279 * * *$ & $127 * * *$ & $286^{* * * *}$ & $34 * * *$ & $280 * * *$ \\
\hline Pseudo- $\mathrm{R}^{2}$ & 0.015 & 0.014 & 0.009 & 0.006 & 0.009 & 0.007 & 0.014 & 0.006 \\
\hline $\mathrm{N}$ & 1,429 & 1,429 & 1,429 & 1,429 & 1,429 & 1,429 & 1,429 & 1,429 \\
\hline
\end{tabular}

Note: Coefficient estimates from CF approach through Tobit estimator is shown, with robust/bootstrap standard errors clustered at household level in parentheses. Bemba and Protestant are the reference groups for Chewa and Tonga, and Catholic, respectively. * $p<0.1 ; * p<0.05 ; * * p<0.01$. 


\section{$5 \quad$ General conclusion and policy implications}

\subsection{Main findings}

In many developing countries, food environments are changing rapidly, with modern food retailers - such as supermarkets and convenience stores - increasingly replacing or complementing traditional food retailers. Unlike traditional food markets and shops, modern retailers often sell a different range of products, at different prices and packaging sizes, and in different shopping atmospheres. Hence, these changing food environments, especially in urban areas, may influence consumers' food choices, dietary quality and affordability, and nutrition. Access and affordability of nutritious diets remain formidable challenges in many developing countries, where hunger and micronutrient malnutrition coexist with overweight and obesity.

Previous research suggested that the growth of modern retailers may contribute to less healthy diets, higher consumption of ultra-processed foods, and rising rates of overweight and obesity among adults. However, previous studies did not pay much attention to the question as to which socioeconomic groups use what type of retailers. Furthermore, the existing research on diet and nutrition effects focused primarily on the role of supermarkets, without accounting for the fact that most consumers obtain their foods from various types of retailers. Furthermore, very few studies analyzed effects of modern retailers on child nutrition, and those that did found mixed results (Umberger et al., 2015; Debela et al., 2020). One shortcoming of all existing studies is that they did not collect individual-level food-intake data. Individual-level dietary data are important to analyze effects of modern retailers on dietary quality (i.e., nutrient intake) and better understand the mechanisms behind the nutrition impacts. Finally, the role of supermarkets on the affordability of recommended nutritious diets was not analyzed in any of the existing studies.

We have addressed the highlighted research gaps by more explicitly analyzing the associations between household socioeconomic status, the use of different types of modern and traditional retailers, and dietary patterns. Using data collected in Lusaka, Zambia, we found that two-thirds of the households use modern and traditional retailers simultaneously. Among the modern retailers, supermarkets account for the largest share of the food purchases. On average, modern retailers account for $42 \%$ of the household food expenditures with notable differences between poor and rich households. Income is an important predictor of the 
use of modern retailers after controlling for other socioeconomic variables. The regression analysis has also shown that using modern retailers is associated with a higher consumption of ultra-processed foods and a lower consumption of unprocessed foods, also after controlling for income and other socioeconomic variables. This is in line with earlier research on the dietary effects of supermarkets (Asfaw, 2008; Rischke et al., 2015). However, unlike earlier studies, we also analyzed the role of other retailers and found that especially the use of traditional grocery stores and neighborhood kiosks is also associated with higher consumption of ultra-processed foods with high content of oils and fats, sugar, and salt. These results suggest that there is a general shift towards the consumption of ultra-processed foods that cannot be attributed to modern retailers alone.

We have also provided the first study that analyzes effects of modern retailers on diets and nutrition with individual-level food-intake and anthropometric data in a developing country. We had collected data from randomly selected households that use modern retailers at different intensities. Individual-level anthropometric and food-intake data were analyzed with control function regression models to estimate effects of modern retailers on body weight, height, dietary diversity, and nutrient intakes. We found that use of modern retailers is positively associated with overweight in adults, but not in children. This is consistent with earlier results for adult populations in Guatemala (Asfaw, 2008) and Kenya (Kimenju et al., 2015; Demmler et al., 2018). For children, we found a positive effect on body height. This is consistent with recent results for children in urban Kenya (Debela et al., 2020). Gains in child height point at likely improvements in dietary quality, even though - due to data limitations this could not be analyzed in previous studies. Furthermore, use of modern retailers increases dietary diversity, as well as calorie, protein, and micronutrient intakes among both adults and children. Effects on protein and micronutrient intakes are channeled primarily through higher consumption of meat and dairy products.

Finally, using food price data and individual-level dietary data from Lusaka, Zambia and control function regression models to account for the likely endogeneity of supermarket purchases, we have analyzed the effects of using supermarkets on the affordability of nutritious diets and dietary quality. We found that on average, the cost of a recommended nutritious diet is US\$1.22 per day, of which the largest share is the cost of starchy staples (68\%), followed by fruits (11\%), and meat, eggs, and fish (9\%). This is consistent with other existing studies in low-income countries (Headey et al., 2019; Hirvonen et al., 2020). 
However, on average, this diet is not affordable to $41 \%$ of low-income group. Furthermore, we found that nutrient-dense foods such as meat, fish, and dairy products are more expensive in supermarkets than in traditional food retailers; largely due to higher quality food products and safety standards. Nevertheless, after controlling for confounding factors such as income and education, we found that buying food in supermarkets increases dietary diversity and intake of nutritious diets, with varying effect sizes among demographic groups. The positive effect of supermarkets on dietary quality is channeled through higher intake of animal source foods such as meat, eggs, fish, and dairy products. This is a welcome finding as it may help to reduce micronutrient malnutrition.

\subsection{Policy implications}

Generally, we find that the growth of modern food retailers influences people's diets and nutrition, and the effects can be positive and negative. These results have a few important policy implications. The positive effects on child nutrition and dietary quality of both children and adults imply that further modernization of food environments should be promoted. However, due to higher quality food products and safety standards, modern retailers - such as supermarkets and convenience stores - offer higher prices for meat, fish, and dairy products than traditional retailers. Therefore, these results suggest a need to shift food policy from focusing on energy-dense foods to affordable healthy diets. Modern retailers could be one of the platforms to make nutritious foods more affordable especially among poor households. Lower prices could come from improvements in local production, higher efficiency in procurements, marketing and trade, and infrastructure developments especially cooling facilities and warehouses. This could help poor consumers to access sufficient quantities of nutritious foods especially animal source foods. Eventually, this could reduce health problems related to micronutrient malnutrition in many developing countries.

Other incentives to offer more healthy foods from modern retailers would be useful policy interventions. For instance, other policy options to consider are regulations related to the advertisement and promotion of healthy foods and their strategic placement within shops. In urban Zambia, the consumption of fresh fruits is particularly low; policies to increase fruit consumption levels would be useful. For instance, a few existing studies (e.g., Glanz et al., 2012; Payne and Niculescu, 2018) have showed that changes in the placement of fruits and vegetables can positively influence consumer choices in developed countries. Related regulations could also be relevant for countries in Africa. 
On the negative side, the effect of increasing adult overweight and obesity associated with modern retailers is undesirable. This is largely due to higher consumption of ultra-processed foods with high contents of fats, added sugars and salts, but poor in micronutrients. Hence, regulatory policies that can help to make food environments healthier would be useful. Possible policy interventions include regulation of advertisement and promotional campaigns for unhealthy foods, regulation of health labels and portion/packaging sizes would be relevant in Zambia and other parts of Africa. Moreover, the results also suggest that modern retailers lead to higher intake of less healthy foods such as sugar, salt, and fat-rich foods in Zambia. Therefore, policy options including taxes on ultra-processed foods and beverages, and incentives to offer more healthy foods, among others (Development Initiative, 2018; Swinburn et al., 2019; Hawkes et al., 2020), would be useful to address different forms of malnutrition in many developing countries.

\subsection{Limitation of the study}

This study has some limitations that should be briefly discussed. First, in the first paper (chapter 2), we used processing level categories, which could not sufficiently classify the degree of healthfulness of a specific food. Second, in the second (chapter 3) and third (chapter 4) papers, we captured individual-level food-intake/dietary data only at one point in time. Hence, we may have systematically missed certain food items that are not consumed on a daily basis. Though more costly, conducting multiple 24-hour dietary recalls could account for the missed food items. Moreover, we could not capture possible seasonal effects of food consumption and nutrition. While seasonality in consumption is expected to be lower in urban than rural areas, it will likely still exist to some extent.

Third, our results are specific for Lusaka in Zambia and should not be generalized. However, it is likely that the diet and nutrition effects of modern retailers may be similar also in other parts of Africa; which are characterized by a rapid modernization of food environment and a high prevalence of different forms of malnutrition concurrently ( $\mathrm{Lu}$ and Reardon, 2018; FAO et al., 2019). Nevertheless, further research is needed to better understand the diet and nutrition effects of changing food environments in different geographical and socioeconomic contexts. Finally, we used cross-sectional data in all three papers, which limits the strength of the identification strategy and the options to analyze dynamic effects. 


\section{References}

Alemu, B., S.A. Block, D. Headey, Y. Bai, and W.A. Masters. 2019. Where are Nutritious Diets most Expensive? Evidence from 195 Foods in 164 Countries. https://www.aeaweb.org/conference/2019/preliminary/paper/RfTTNfGY.

Andersson, C.I.M., C.G.K. Chege, E.J.O. Rao, and M. Qaim. 2015. Following Up on Smallholder Farmers and Supermarkets in Kenya. American Journal of Agricultural Economics 97 (4): 1247-1266.

Arimond, M., D., Wiesmann, E., Becquey, et al., 2010. Simple Food Group Diversity Indicators Predict Micronutrient Adequacy of Women's Diets in 5 Diverse, ResourcePoor Settings. Journal of Nutrition 140 (11): 2059S-2069S.

Asfaw, A. 2008. Does Supermarket Purchase Affect the Dietary Practices of Households? Some Empirical Evidence from Guatemala. Development Policy Review 26 (2): 227-243. Asfaw, A. 2011. Does Consumption of Processed Foods Explain Disparities in the Body Weight of Individuals? The Case of Guatemala. Health Economics 20 (2): 184-195.

Beatty, T.K.M., B.H. Lin, and T.A. Smith. 2014. Is Diet Quality Improving? Distribution Changes in the United States, 1989-2008. American Journal of Agricultural Economics 96 (3): 769-789.

Berger, M., and B. van Helvoirt. 2018. Ensuring Food Secure Cities - Retail Modernization and Policy Implications in Nairobi, Kenya. Food Policy 79: 12-22.

Black, R. E., C.G. Victora, S.P., Walker, et al. 2013. Maternal and Child Undernutrition and Overweight in Low-Income and Middle-Income Countries. Lancet 382: 427-451.

Cappellari, L., and S.P. Jenkins. 2003. Multivariate Probit Regression Using Simulated Maximum Likelihood. The Stata Journal 3 (3): 278-294.

Colen, L., P.C. Melo, Y. Abdul-Salam, D. Roberts, S. Mary, S. Gomez, and Y. Paloma. 2018. Income Elasticities for Food, Calories and Nutrients across Africa: A Meta-analysis. Food Policy 77: 116-132.

Cost of Nutritious Diets Consortium. 2018. Indicators and Tools for the Cost of Nutritious Diets. Boston, MA: Tuffs University.

Courtemanche, C., A. Carden, X. Zhou, and M. Ndirangu. 2019. Do Walmart Supercenters Improve Food Security? Applied Economic Perspectives and Policy 41 (2): 177-198.

Debela, B.L., K.M., Demmler, S. Klasen, and M. Qaim. 2020. Supermarket Food Purchases and Child Nutrition in Kenya. Global Food Security. https://doi.org/10.1016/j.gfs.2019.100341. 
Demmler, K.M., O. Ecker, and M. Qaim. 2018. Supermarket Shopping and Nutritional Outcomes: A Panel Data Analysis for Urban Kenya. World Development 102: 292-303.

Demmler, K.M., S. Klasen, J.M. Nzuma, and M. Qaim. 2017. Supermarket Purchase Contributes to Nutrition-related Non-communicable Diseases in Urban Kenya. PLoS One 12: $\mathrm{e} 0185148$.

Development Initiatives. 2018. Global Nutrition Report 2018. Bristol: Development Initiatives.

Di Falco, S., M. Veronesi, and M. Yesuf. 2011. Does Adaptation to Climate Change Provide Food Security? A Micro-perspective from Ethiopia. American Journal of Agricultural Economics 93: 829-846.

Dizon, F., A. Herforth, and Z. Wang. 2019. The Cost of a Nutritious Diet in Afghanistan, Bangladesh, Pakistan, and Sri Lanka. Global Food Security 21: 38-51.

FAO and FHI 360. 2016. Minimum Dietary Diversity for Women: A Guide for Measurement. Rome, FAO.

FAO and Government of Kenya. 2018. Kenya Food Composition Tables. Nairobi, 254 pp. http://www.fao.org/3/I9120EN/i9120en.pdf.

FAO, IFAD, UNICEF, WFP and WHO. 2019. The State of Food Security and Nutrition in the World 2019: Safeguarding against Economic Slowdowns and Downturns. Rome, FAO.

FAO. 2001. Human Energy Requirements. Report of a Joint FAO/WHO/UNU Expert Consultation, Food and Nutrition Technical report No. 1. Rome, FAO.

Figuié, M., and P. Moustier. 2009. Market Appeal in an Emerging Economy: Supermarkets and Poor Consumers in Vietnam. Food Policy 34: 210-217.

Fongar, A., T. Gödecke, and M. Qaim. 2019. Various Forms of Double Burden of Malnutrition Problems Exist in Rural Kenya. BMC Public Health 19: 1543.

Freire, T., and S. Rudkin. 2019. Healthy Food Diversity and Supermarket Interventions: Evidence from the Seacroft Intervention Study. Food Policy 83: 125-138.

Glanz, K., M.D. Bader, and S. Iyer. 2012. Retail Grocery Store Marketing Strategies and Obesity: An Integrative Review. American Journal of Preventive Medicine 42 (5): 503512.

Global Alliance for Improved Nutrition (GAIN). 2020. Animal-source Foods for Human and Planetary Health: GAIN's Position. GAIN. Briefing Paper Series \#2. Geneva, Switzerland. 
Godfray, H.C.J., P. Aveyard, T. Garnett, J.W. Hall, T.J. Key, J. Lorimer, R.T. Pierrehumbert, P. Scarborough, M. Springmann, and S.A. Jebb. 2018. Meat Consumption, Health, and the Environment. Science 361 (6399): eaam5324.

Gómez, M.I., and K.D. Ricketts. 2013. Food Value Chain Transformations in Developing Countries: Selected Hypotheses on Nutritional Implications. Food Policy 42: 139-150.

Gorton, M., J. Sauer, and P. Supatpongkul. 2011. Wet Markets, Supermarkets and the "Big Middle" for Food Retailing in Developing Countries: Evidence from Thailand. World Development 39 (9): 1624-1637.

Harris, J., B. Chisanga, S. Drimie, and G. Kennedy. 2019. Nutrition Transition in Zambia: Changing Food Supply, Food Prices, Household Consumption, Diet and Nutrition Outcomes. Food Security 11 (2): 371-387.

Hawkes, C. 2008. Dietary Implications of Supermarket Development: A Global Perspective. Development Policy Review 26 (6): 657-692.

Hawkes, C., M.T. Ruel, M. Salm, B. Sinclair, and F. Branca. 2020. Double-duty Actions: Seizing Programme and Policy Opportunities to Address Malnutrition in all its Forms. Lancet 395: 142-155.

Headey, D., and H. Alderman. 2019. The Relative Caloric Prices of Healthy and Unhealthy Foods Differ Systematically across Income Levels and Continents. Journal of Nutrition 149 (11): 2020-2033.

Headey, D., K. Hirvonen, and J. Hoddinott. 2018. Animal Sourced Foods and Child Stunting. American Journal of Agricultural Economics 100: 1302-1319.

Headey, D., K. Hirvonen, J. Hoddinott, and D. Stifel. 2019. Rural Food Markets and Child Nutrition. American Journal of Agricultural Economics 101 (5): 1311-1327.

Hirvonen, K., Y. Bai, D. Headey, and W.A. Masters. 2020. Affordability of the EAT-Lancet Reference Diet: A Global Analysis. Lancet Global Health 8 (1): e59-66.

HLPE. 2017. Nutrition and Food Systems; Report of the High Level Panel of Experts on Food Security and Nutrition. Rome: Committee on World Food Security.

Hovhannisyan, V., C. Cho, and M. Bozic. 2019. The Relationship between Price and Retail Concentration: Evidence from the US Food Industry. European Review of Agricultural Economics 46: 319-345.

IFPRI. 2017. Global Nutrition Report 2017. Washington, DC: International Food Policy Research Institute 
Institute of Medicine (IOM). 2002. Dietary Reference Intakes for Energy, Carbohydrate,

Fiber, Fat, Fatty Acids, Cholesterol, Protein, and Amino acids. Washington DC: The National Academies Press.

Institute of Medicine (IOM). 2006. Dietary Reference Intakes: The Essential Guide to Nutrient Requirements. Washington DC: The National Academies Press.

International Zinc Nutrition Consultative Group (IZiNCG), K.H. Brown, J.A. Rivera, et al. 2004. International Zinc Nutrition Consultative Group Technical Document \#1. Assessment of the Risk of Zinc Deficiency in Populations and Options for its Control. Food Nutrition Bulletin 25: S99-S203.

Kaliwile, C., C. Michelo, T.J. Titcomb, M. Moursi, M.D. Angel, C. Reinberg, P. Bwembya, R. Alders, and S.A. Tanumihardjo. 2019. Dietary Intake Patterns among Lactating and Non-lactating Women of Reproductive Age in Rural Zambia. Nutrients 11 (2): 288.

Kelly, M., S.A. Seubsman, C. Banwell, J. Dixon, and A. Sleigh. 2014. Thailand's Food Retail Transition: Supermarket and Fresh Market Effects on Diet Quality and Health. British Food Journal 116: 1180-1193.

Kennedy, G., T. Ballard, and M.C. Dop. 2011. Guidelines for Measuring Household and Individual Dietary Diversity. Food and Agriculture Organization of the United Nations, Rome.

Khonje, M.G., and M. Qaim. 2019. Modernization of African Food Retailing and (Un)healthy Food Consumption. Sustainability 11 (16): 4306.

Kimenju, S.C., R. Rischke, S. Klasen, and M. Qaim. 2015. Do Supermarkets Contribute to the Obesity Pandemic in Developing Countries? Public Health Nutrition 18: 3224-3233.

Kroll, F., E.C. Swart, R.A. Annan, A.M. Thow, et al. 2019. Mapping Obesogenic Food Environments in South Africa and Ghana: Correlations and Contradictions. Sustainability 11: 3924 .

Laska, M.N., L.S. Sindberg, G.X. Ayala, H. D’Angelo, L.A. Horton, K.M. Ribisl, A. Kharmats, C. Olson, and J. Gittelsohn. 2018. Agreements between Small Food Store Retailers and their Suppliers: Incentivizing Unhealthy Foods and Beverages in Four Urban Settings. Food Policy 79: 324-330.

Lu, L., and T. Reardon. 2018. An Economic Model of the Evolution of Food Retail and Supply Chains from Traditional Shops to Supermarkets to E-commerce. American Journal of Agricultural Economics 100 (5): 1320-1335. 
Masters, W.A., Y. Bai, A. Herforth, D.B. Sarpong, F. Mishili, J. Kinabo, and J.C. Coates. 2018. Measuring the Affordability of Nutritious Diets in Africa: Price Indexes for Diet Diversity and the Cost of Nutrient Adequacy. American Journal of Agricultural Economics 100 (5): 1285-1301.

Mergenthaler, M., K. Weinberger, and M. Qaim. 2009. The Food System Transformation in Developing Countries: A Disaggregate Demand Analysis for Fruits and Vegetables in Vietnam. Food Policy 34: 426-436.

Minten, B., and T. Reardon. 2008. Food Prices, Quality, and Quality's Pricing in Supermarkets versus Traditional Markets in Developing Countries. Applied Economic Perspectives and Policy 30 (3): 480-490.

Minten, B., T. Reardon, and K.Z. Chen. 2017. Agricultural Value Chains: How Cities Reshape Food Systems. In 2017 Global Food Policy Report. Chapter 5: 42-49. Washington, DC: International Food Policy Research Institute (IFPRI).

Minten, B., T. Reardon, and R. Sutradhar. 2010. Food Prices and Modern Retail: The Case of Delhi. World Development 38 (12): 1775-1787.

Monteiro, C.A., R.B. Levy, M.R. Claro, I. Ribeiro, Rugani., de Castro, and G. Cannon. 2010. Increasing Consumption of Ultra-processed Foods and likely Impact on Human Health: Evidence from Brazil. Public Health Nutrition 14 (1): 5-13.

Nyirenda, D.B., M. Musukwa, R. Habulembe, and J. Shindano. 2009. Zambia Food Composition Tables. $4^{\text {th }}$ edition. Lusaka, Zambia.

Odunitan-Wayas, F., K. Okop, R. Dover, O. Alaba, et al., 2018. Food Purchasing Characteristics and Perceptions of Neighborhood Food Environment of South Africans Living in Low-, Middle- and High-Socioeconomic Neighborhoods. Sustainability 10: 4801.

Payne, C., and M. Niculescu. 2018. Can Healthy Checkout End-caps Improve Targeted Fruit and Vegetable Purchases? Evidence from Grocery and SNAP Participant Purchases. Food Policy 79: 318-323.

PlanetRetail. 2017. Country Breakdown by Retailer. Planet Retail, retrieved April 10, 2017, from http://www.planetretail.net/.

Popkin, B.M. 2014. Nutrition, Agriculture and the Global Food System in Low and Middle Income Countries. Food Policy 47: 91-96.

Popkin, B.M. 2017. Relationship between Shifts in Food System Dynamics and Acceleration of the Global Nutrition Transition. Nutrition Reviews 75 (2): 73-82. 
Popkin, B.M., and T. Reardon. 2018. Obesity and the Food System Transformation in Latin America. Obesity Reviews 19: 1028-1064.

Popkin, B.M., C. Corvalan, and L.M. Grummer-Strawn. 2020. Dynamics of the Double Burden of Malnutrition and the Changing Nutrition Reality. Lancet 395: 65-74.

Qaim, M. 2017. Globalisation of Agrifood Systems and Sustainable Nutrition. Proceedings of the Nutrition Society 76 (1): 12-21.

Reardon, T., and C.P. Timmer. 2014. Five Inter-linked Transformations in the Asian Agrifood Economy: Food Security Implications. Global Food Security 3 (2): 108-117.

Reardon, T., C.P. Timmer, C.B. Barrett, and J.A. Berdegué. 2003. The Rise of Supermarkets in Africa, Asia, and Latin America. American Journal of Agricultural Economics 85 (5): $1140-1146$.

Reardon, T., R. Echeverria, J. Berdegué, B. Minten, S. Liverpool-Tasie, D. Tschirley, and D. Zilberman. 2019. Rapid Transformation of Food Systems in Developing Regions: Highlighting the Role of Agricultural Research and Innovations. Agricultural Systems 172: $47-59$.

Rischke, R., S.C. Kimenju, S. Klasen, and M. Qaim. 2015. Supermarkets and Food Consumption Patterns: The Case of Small Towns in Kenya. Food Policy 52: 9-21.

Ruel, M.T., and H. Alderman. 2013. Nutrition-sensitive Interventions and Programmes: How Can They Help to Accelerate Progress in Improving Maternal and Child Nutrition? Lancet 382: 536-551.

Ruel, M.T., J. Garrett, and S. Yosef. 2017. Food Security and Nutrition: Growing Cities, New Challenges. In 2017 Global Food Policy Report, Chapter 3: 24-33. Washington, DC: IFPRI.

Rupa, A.J., W.J. Umberger, and D. Zeng. 2019. Does Food Market Modernisation Lead to Improved Dietary Diversity and Diet Quality for Urban Vietnamese Households? Australian Journal of Agricultural and Resource Economics 63: 499-520

Sanchez, P.A. 2020. Viewpoint: Time to Increase Production of Nutrient-rich Foods. Food Policy 91: 101843.

Schipmann, C., and M. Qaim. 2011. Modern Food Retailers and Traditional Markets in Developing Countries: Comparing Quality, Prices, and Competition Strategies in Thailand. Applied Economic Perspectives and Policy 33: 345-362.

Shoprite Holdings. 2017. Financial Results for the Year Ended 2 July 2017. Johannesburg. https://www.shopriteholdings.co.za/investor-centre.html (Accessed April 2, 2019). 
Springmann, M., et al. 2018. Health and Nutritional Aspects of Sustainable Diet Strategies and their Association with Environmental Impacts: A Global Modelling Analysis with Country-level Detail. The Lancet Planetary Health 2 (10): e451-e461.

Stewart, H., and D. Dong. 2018. How Strong is the Demand for Food through Direct-toConsumer Outlets? Food Policy 79: 35-43.

Steyn, N.P., and Z.J. Mchiza. 2014. Obesity and the Nutrition Transition in Sub-Saharan Africa. Annals of the New York Academy of Sciences 1311 (1): 88-101.

Steyn, N.P., J.H. Nel, G. Nantel, G. Kennedy, and D. Labadarios. 2006. Food Variety and Dietary Diversity Scores in Children: Are They Good Indicators of Dietary Adequacy? Public Health Nutrition 9: 644-650.

Suryadarma, D., A.P. Akhmadi, S. Budiyati, M. Rosfadhila, and A. Suryahadi. 2010. Traditional Food Traders in Developing Countries and Competition from Supermarkets: Evidence from Indonesia. Food Policy 35 (1): 79-86.

Swinburn, B.A., V.I. Kraak, S. Allender, et al. 2019. The Global Syndemic of Obesity, Undernutrition, and Climate Change: The Lancet Commission report. Lancet 393: 791846.

Tessier, S., P. Traissac, B. Maire, et al. 2008. Regular Users of Supermarkets in Greater Tunis Have a slightly Improved Diet Quality. Journal of Nutrition 138: 768-774.

Torheim, L.E., F. Ouattara, M.M. Diarra, F.D. Thiam, I. Barikmo, A. Hatloy, and A. Oshaug. 2004. Nutrient Adequacy and Dietary Diversity in Rural Mali: Association and Determinants. European Journal of Clinical Nutrition 58 (4): 594-604.

Tschirley, D., T. Reardon, M. Dolislager, and J. Snyder. 2015. The Rise of a Middle Class in East and Southern Africa: Implications for Food System Transformation. Journal of International Development 27: 628-646.

U.S. Department of Agriculture (USDA). 2019. Healthy Eating Index. The Center for Nutrition Policy and Promotion, 2019. Available online: https://www.fns.usda.gov/howhei-scored.

Umberger, W.J., X. He, N. Minot, and H. Toiba. 2015. Examining the Relationship between the Use of Supermarket and Over-nutrition in Indonesia. American Journal of Agricultural Economics 97 (2): 510-525.

Vetter, T., M.N. Larsen, and T.B. Bruun. 2019. Supermarket-led Development and the Neglect of Traditional Food Value Chains: Reflections on Indonesia's Agri-food System Transformation. Sustainability 11: 498. 
Villa, K.M., C.B. Barrett, and D.R. Just. 2011. Whose Fast and Whose Feast? Intrahousehold Asymmetries in Dietary Diversity Response among East African Pastoralists. American Journal of Agricultural Economics 93 (4): 1062-1081.

Wertheim-Heck, S.C.O., S. Vellema, and G. Spaargaren. 2015. Food Safety and Urban Food Markets in Vietnam: The Need for Flexible and Customized Retail Modernization Policies. Food Policy 54: 95-106.

WHO and FAO. 2004. Vitamin and Mineral Requirements in Human Nutrition. 2nd edn. Geneva: World Health Organization.

WHO. 2006. Multicentre Growth Reference Study Group. WHO Child Growth Standards: Length/Height-for-Age, Weight-for-Age, Weight-for-Length, Weight-for-Height and Body Mass Index-for-Age: Methods and Development. Geneva: World Health Organization.

WHO. 2010. Global Status Report on Non-communicable Diseases. World Health Organization. Italy.

Willett, W., et al. 2019. Food in the Anthropocene: The EAT-Lancet Commission on Healthy Diets from Sustainable Food Systems. The Lancet 393 (10170): 447-492.

Wooldridge, J.M. 2010. Econometric Analysis of Cross-section and Panel Data. 2nd Ed. Cambridge, MA: MIT Press.

Wooldridge, J.M. 2015. Control Function Methods in Applied Econometrics. Journal of Human Resources 50: 420-445.

World Bank. 2019. Poverty-World Bank Open Data-World Bank Group. https://data.worldbank.org/topic/poverty (Accessed August 3, 2019).

Zhong, T., Z. Si, J. Crush, Z. Xu, X. Huang, S. Scott, S. Tang, and X. Zhang. 2018. The Impact of Proximity to Wet Markets and Supermarkets on Household Dietary Diversity in Nanjing City, China. Sustainability 10: 1465.

Ziba, F., and M. Phiri. 2017. The Expansion of Regional Supermarket Chains: Implications for Local Suppliers in Zambia. WIDER Working Paper, No. 2017/58, ISBN 978-929256-282-3. The United Nations University-World Institute for Development Economics Research: Helsinki, Finland. 


\section{General appendix: Survey questionnaire}

\section{CAN MODERN FOOD RETAILERS IMPROVE DIETS AND NUTRITION IN URBAN AFRICA? EMPIRICAL EVIDENCE FROM ZAMBIA}

\section{Household Consumption and Expenditure Survey for Urban Zambia 2018}

The National Food and Nutrition Commission of Zambia and University of Goettingen in Germany are carrying out a research on modernization of the food retail environment and nutrition transition. We are currently doing a survey which aims to analyze whether the modernization of the food retail environment in urban Africa, with its growth of supermarkets and other modern food outlets, is influencing consumer food choices, dietary quality, and nutrition. Your participation in answering these questions is very much appreciated. Your responses will be COMPLETELY CONFIDENTIAL and will only be used for purpose of this study.

\section{CONTENTS}

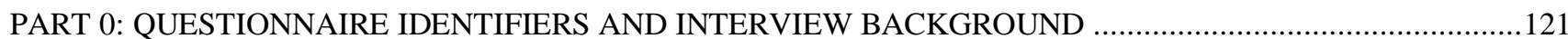

PART 1: HOUSEHOLD COMPOSITION AND CHARACTERISTICS .............................................................

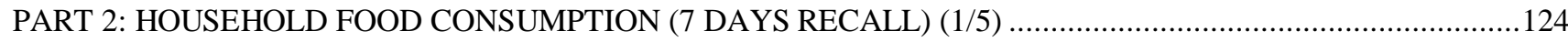

PART 3: ACCESS TO FOOD OUTLETS, HEALTH FACILTIES AND SHOPPING BEHAVIOUR .......................129

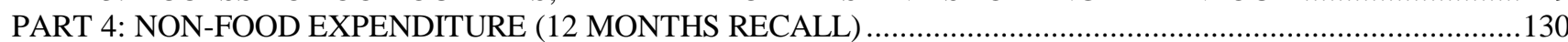

PART 5: INCOME SOURCES, TRANSFERS AND HOUSEHOLD INCOME ..................................................131

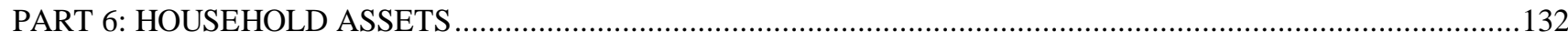

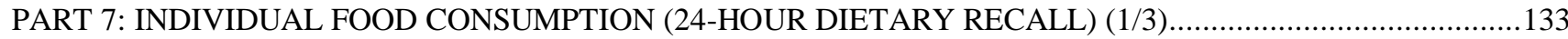

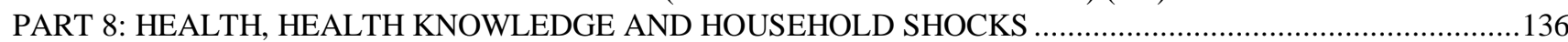

PART 9: ANTHROPOMETRIC MEASUREMENTS, PHYSICAL AND LEISURE ACTIVITIES ..........................137

\section{PART 0: QUESTIONNAIRE IDENTIFIERS AND INTERVIEW BACKGROUND}

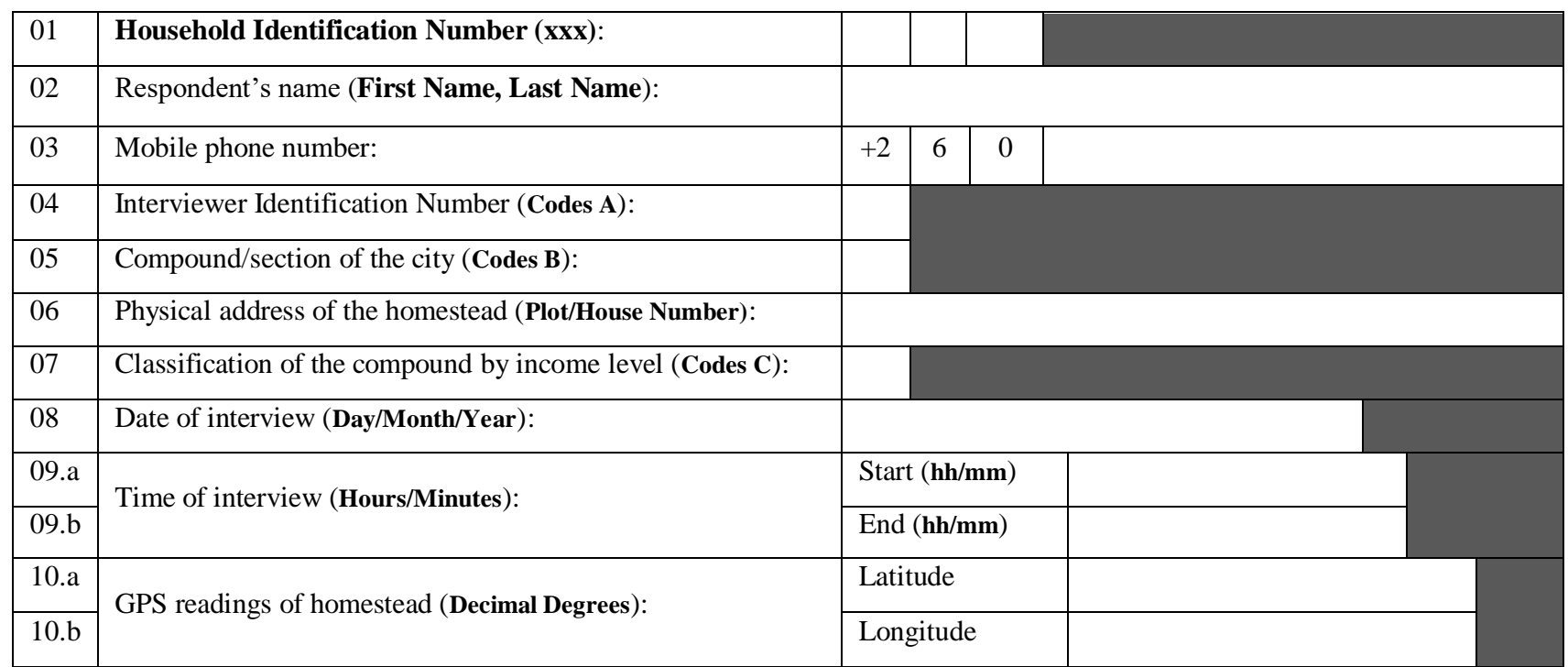

\begin{tabular}{|c|c|c|c|}
\hline $\begin{array}{l}\text { Codes A } \\
\text { 1. Brian Mpande } \\
\text { 2. } \\
\text { 3. Eric Chikwalila } \\
\text { 4. } \text { Kelvine Kaango Sinyinza } \\
\text { 5. Samba Manjolo } \\
\text { 6. } \\
\text { Mully Phiri }\end{array}$ & $\begin{array}{l}\text { Codes B } \\
\text { 1. Avodale } \\
\text { 2. Chalala } \\
\text { 3. Chelston } \\
\text { 4. Chilenje } \\
\text { 5. Gardens } \\
\text { 6. Kawata } \\
\text { 7. Kaunda square } \\
\text { 8. Kalingalinga } \\
\text { 9. Mtendere }\end{array}$ & $\begin{array}{l}\text { 10. Ngombe } \\
\text { 11. North Mead } \\
\text { 12. PHI } \\
\text { 13. Woodlands }\end{array}$ & $\begin{array}{r}\text { Codes C } \\
\text { 1. High } \\
\text { 2. Middle } \\
\text { 3. Low }\end{array}$ \\
\hline
\end{tabular}


We are researchers from the National Food and Nutrition Commission of Zambia and University of Goettingen in Germany. We are currently doing a survey which aims to analyze whether the modernization of the food retail environment in urban Africa, with its growth of supermarkets and other modern food outlets, is influencing consumer food choices, dietary quality, and nutrition. The knowledge can help to better understand the wider implications of the observed transformation of food systems and to guide policies aimed at reducing malnutrition. The survey includes questions about the household generally - economic, social, and demographic data (employment, income, food consumption, education, age etc.), and questions specific to some individuals i.e., food consumption within your household. We will also take body size measurements height, weight, and waist circumference - of adults and children. Your participation in answering these questions is very much appreciated. Your responses will be COMPLETELY CONFIDENTIAL and will only be used for purpose of this study.

Do you have any questions about the survey or what I have said? Do you agree to participate in the survey, including the interviews and the anthropometric measurements of adults and children? If yes, let the potential respondent sign this form, even on behalf of other members of household especially children. May we begin the interview then?

I hereby confirm that I have adequately received information about the study and I understood the purpose of the study and procedure of measurements that will be taken in the survey: "Can modern food retailers improve diets and nutrition in urban Africa? Empirical evidence from Zambia”.

Yes

No

I had enough opportunity to ask questions about the study and all my questions have been answered in a satisfactory way.

Yes

No

I agree that my body size - height, weight, and waist circumference - will be measured and that all my personal data will be treated confidentially. The data will be used for the purpose of this research study only. I agree that my personal data are stored and handled in accordance with the Lower Saxony and Federal German data privacy act.

Yes

No

I feel completely informed and agree to the participation in the study "Can modern food retailers improve diets and nutrition in urban Africa? Empirical evidence from Zambia”.

Compound/Section of the city:

Date: .$/ 2018$

(1)

Name of the participant

$\overline{\text { Signature of participant }}$

Signature of interviewer
(2)

Name of the interviewer $\overline{\text { Family Member_ID }}$ 


\section{PART 1: HOUSEHOLD COMPOSITION AND CHARACTERISTICS}

Notes: Total household size is the filter in this section

\begin{tabular}{|c|c|c|c|c|c|c|c|c|c|}
\hline 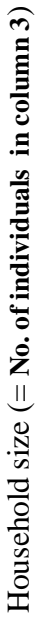 & 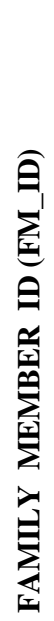 & $\begin{array}{c}\text { Name ( First Name, Last Name ) } \\
\text { of household member } \\
\text { (start with head of the household/respondent) }\end{array}$ & 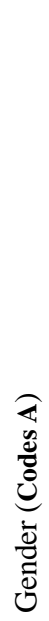 & 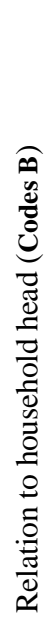 & 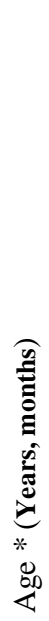 & 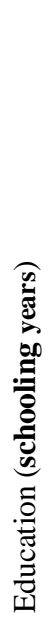 & 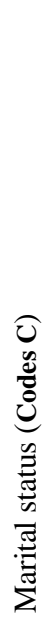 & 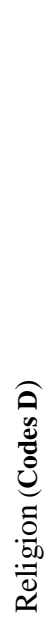 & 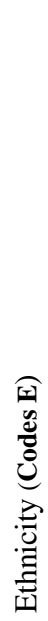 \\
\hline 1 & 2 & 3 & 4 & 5 & 6 & 7 & 8 & 9 & 10 \\
\hline & 01 & & & & & & & & \\
\hline & 02 & & & & & & & & \\
\hline & 03 & & & & & & & & \\
\hline & 04 & & & & & & & & \\
\hline & 05 & & & & & & & & \\
\hline & 06 & & & & & & & & \\
\hline & 07 & & & & & & & & \\
\hline & 08 & & & & & & & & \\
\hline & 09 & & & & & & & & \\
\hline & 10 & & & & & & & & \\
\hline & 11 & & & & & & & & \\
\hline & 12 & & & & & & & & \\
\hline & 13 & & & & & & & & \\
\hline & 14 & & & & & & & & \\
\hline & 15 & & & & & & & & \\
\hline
\end{tabular}

Notes: * For the under 5 year olds, ask month, day and year born and then compute the age yourself (in one decimal places).

\begin{tabular}{|c|c|c|c|c|c|}
\hline $\begin{array}{l}\text { Codes A } \\
0 . \text { Female } \\
\text { 1. Male }\end{array}$ & $\begin{array}{l}\text { Codes B } \\
\text { 1. Household head } \\
\text { 2. Spouse/Partner } \\
\text { 3. Son/Daughter } \\
\text { 4. Brother/Sister } \\
\text { 5. Brother/Sister in-law } \\
\text { 6. Parent (Mother/Father) } \\
\text { 7. Grand children } \\
\text { 8. Grand parents } \\
\text { 9. Step children } \\
\text { 10. House maid/Garden boy } \\
\text { 11. Other relative } \\
\text { 12. Non-relative }\end{array}$ & $\begin{array}{l}\text { Codes C } \\
\text { 1. Married living with Spouse/Husband } \\
\text { 2. Married but Spouse/Husband away } \\
\text { 3. Divorced/Separated } \\
\text { 4. Widow/Widower } \\
\text { 5. Single } \\
\text { 6. Too young to be married }\end{array}$ & $\begin{array}{l}\text { Codes D } \\
\text { 1. Protestant } \\
\text { 2. Catholic } \\
\text { 3. SDA } \\
\text { 4. Muslim } \\
\text { 5. Jewish } \\
\text { 6. Traditionalist } \\
\text { 7. Others } \\
\text { 8. No religion }\end{array}$ & $\begin{array}{l}\text { Codes E } \\
\text { 1. Bemba } \\
\text { 2. Chewa } \\
\text { 3. Goba } \\
\text { 4. Kaonde } \\
\text { 5. Lala } \\
\text { 6. Lozi } \\
\text { 7. Lunda } \\
\text { 8. Luvale } \\
\text { 9. Mbunda } \\
\text { 10. Ngoni } \\
\text { 11. Nsenga }\end{array}$ & $\begin{array}{r}\text { Codes E } \\
\text { 12. Tonga } \\
\text { 13. Tumbuka } \\
\text { 14. Others }\end{array}$ \\
\hline
\end{tabular}


Here, wife and/or the person involved in purchases should be the principal respondent/s

Notes: Please include all foods consumed by all household members.

\begin{tabular}{|c|c|c|c|c|c|c|c|c|}
\hline \multirow[b]{2}{*}{ 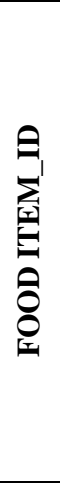 } & \multirow[b]{2}{*}{ FOOD ITEM } & \multirow[b]{2}{*}{ 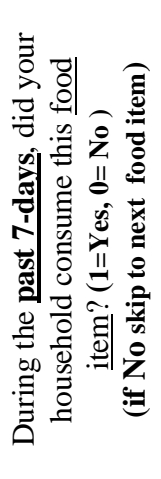 } & \multicolumn{6}{|c|}{ Food consumption expenditure in the past 7 -days } \\
\hline & & & $\begin{array}{c}\text { How } \\
\text { much in } \\
\text { total was } \\
\text { consumed } \\
\text { by the HH } \\
\text { during last } \\
7 \text { days? } \\
\text { (Qty) }\end{array}$ & 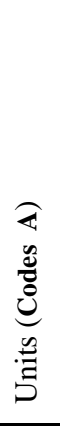 & $\begin{array}{c}\text { Average } \\
\text { price per } \\
\text { unit } \\
(\mathbf{Z M K})\end{array}$ & $\begin{array}{l}\text { Total cost of } \\
\text { food item } \\
\text { (ZMK) }\end{array}$ & $\begin{array}{c}\text { Where exactly } \\
\text { did your } \\
\text { household } \\
\text { purchase or } \\
\text { source this } \\
\text { food item? } \\
\text { ( Codes B) }\end{array}$ & $\begin{array}{l}\text { Which } \\
\text { month is this } \\
\text { food item } \\
\text { cheapest/ } \\
\text { plenty? } \\
\text { (Codes C) }\end{array}$ \\
\hline 1 & 2 & 3 & 4 & 5 & 6 & 7 & 8 & 9 \\
\hline $\mathbf{A}$ & Cereals & & & & & & & \\
\hline 01 & Maize (Dry) & & & & & & & \\
\hline 02 & Maize (Flour) & & & & & & & \\
\hline 03 & Maize (Green) & & & & & & & \\
\hline 04 & Rice (White) & & & & & & & \\
\hline 05 & Rice (Brown) & & & & & & & \\
\hline 06 & Wheat (Flour) & & & & & & & \\
\hline 07 & Sorghum & & & & & & & \\
\hline 08 & Millet & & & & & & & \\
\hline 09 & Porridge mix & & & & & & & \\
\hline 10 & Cornflakes & & & & & & & \\
\hline 11 & Oats & & & & & & & \\
\hline 12 & Bread (White) & & & & & & & \\
\hline 13 & Bread (Brown) & & & & & & & \\
\hline 14 & Wheat buns (White) & & & & & & & \\
\hline 15 & Wheat buns (Brown) & & & & & & & \\
\hline 16 & Pasta (Spaghetti) & & & & & & & \\
\hline 17 & Other cereals & & & & & & & \\
\hline B & Roots and Tubers & & & & & & & \\
\hline 18 & Irish potatoes & & & & & & & \\
\hline 19 & Sweet potatoes & & & & & & & \\
\hline 20 & Cassava (Tuber) & & & & & & & \\
\hline 21 & Cassava (Flour) & & & & & & & \\
\hline 22 & Banana/Plantains (Boiled) & & & & & & & \\
\hline 23 & Pumpkins & & & & & & & \\
\hline 24 & Yams & & & & & & & \\
\hline 25 & $\begin{array}{l}\text { Other roots and tubers } \\
\text { (Specify) }\end{array}$ & & & & & & & \\
\hline $\mathbf{C}$ & Legumes (1/2) & & & & & & & \\
\hline 26 & Beans dry & & & & & & & \\
\hline 27 & Beans fresh & & & & & & & \\
\hline 28 & Groundnut dry & & & & & & & \\
\hline 29 & Groundnut fresh & & & & & & & \\
\hline
\end{tabular}

\begin{tabular}{|c|c|c|c|}
\hline Codes A & Codes A & Codes B & Codes C \\
\hline 1. Teaspoon & 10. Kilogram & 1. Superstore/Hyper store & 0 . Almost the same in a year \\
\hline 2. Tablespoon & 11. Number & 2. Supermarket & 1. January \\
\hline 3. Slice of bread & 12. Lump of Nshima & 3. Convenience store & 2. February \\
\hline 4. Piece & 13. Regular (Small) Chips & 4. Grocery store & 3. March \\
\hline 5. Coffee/Tea cup & 14. Pizza box (Small) & 5. Wet (Soweto) market & 4. April \\
\hline 6. Cup (Standard) & 15. Cone (Ice cream) & 6. Traditional/Compound market & 5. May \\
\hline 7. Milliliter & 16. Loaf of Bread & 7. Neighborhood kiosks & 6. June \\
\hline 8. Liter & 17. Packet (Sugar..etc) & 8. Roadside market & 7. July \\
\hline \multirow[t]{6}{*}{ 9. Gram } & 18. Heap & 9. Weekly market & 8. August \\
\hline & 19. Bunch & 10. Fast food restaurant & 9. September \\
\hline & 20. Meda & 11. Casual/Ordinary restaurant & 10. October \\
\hline & 21. Bp & 12. Own production & 11. November \\
\hline & 22. Bottle/can & 13. Gift/Free food & 12. December \\
\hline & 23. Crate & 14. Others, specify...... & 13. Not applicable \\
\hline
\end{tabular}


Here, wife and/or the person involved in purchases should be the principal respondent/s

Notes: Please include all foods consumed by all household members.

\begin{tabular}{|c|c|c|c|c|c|c|c|c|c|}
\hline \multirow[b]{2}{*}{ 空 } & \multirow{2}{*}{\multicolumn{2}{|c|}{ FOOD ITEM }} & \multirow[b]{2}{*}{ 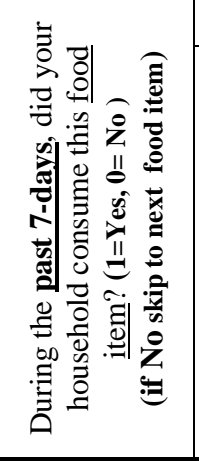 } & \multicolumn{6}{|c|}{ Food consumption expenditure in the past 7-days } \\
\hline & & & & $\begin{array}{c}\text { How } \\
\text { much in } \\
\text { total was } \\
\text { consumed } \\
\text { by the HH } \\
\text { during last } \\
7 \text { days? } \\
\text { (Qty) }\end{array}$ & 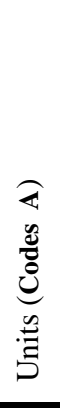 & $\begin{array}{c}\text { Average } \\
\text { price per } \\
\text { unit } \\
(\mathbf{Z M K})\end{array}$ & $\begin{array}{l}\text { Total cost of } \\
\text { food item } \\
(\mathbf{Z M K})\end{array}$ & $\begin{array}{c}\text { Where exactly } \\
\text { did your } \\
\text { household } \\
\text { purchase or } \\
\text { source this } \\
\text { food item? } \\
\text { ( Codes B) }\end{array}$ & $\begin{array}{l}\text { Which } \\
\text { month is this } \\
\text { food item } \\
\text { cheapest/ } \\
\text { plenty? } \\
\text { (Codes C) }\end{array}$ \\
\hline 1 & 2 & & 3 & 4 & 5 & 6 & 7 & 8 & 9 \\
\hline $\mathbf{C}$ & \multicolumn{9}{|l|}{ Legumes (2/2) } \\
\hline 30 & \multicolumn{9}{|l|}{ Cowpea dry grain } \\
\hline 31 & \multicolumn{9}{|l|}{ Cowpea fresh grain } \\
\hline 32 & \multicolumn{9}{|l|}{ Greengram (Mphodza) } \\
\hline 33 & \multicolumn{9}{|l|}{ Pigeonpea dry } \\
\hline 34 & \multicolumn{9}{|l|}{ Pigeonpea fresh } \\
\hline 35 & \multicolumn{9}{|l|}{ Soybean } \\
\hline 36 & \multicolumn{9}{|l|}{ Velvet beans (Nzama) } \\
\hline 37 & \multicolumn{9}{|l|}{ Other legumes (Specify) } \\
\hline D & \multicolumn{9}{|l|}{ Meat and Fish } \\
\hline 38 & \multicolumn{9}{|l|}{ Beef } \\
\hline 39 & \multicolumn{9}{|l|}{ Sausage-(Beef) } \\
\hline 40 & \multicolumn{9}{|l|}{ Chicken } \\
\hline 41 & \multicolumn{9}{|l|}{ Sausage-(Chicken) } \\
\hline 42 & \multicolumn{9}{|l|}{ Goat meat } \\
\hline 43 & \multicolumn{9}{|l|}{ Pig meat } \\
\hline 44 & \multicolumn{9}{|l|}{ Sausage-(Pork) } \\
\hline 45 & \multicolumn{9}{|l|}{ Soymeat } \\
\hline 46 & \multicolumn{9}{|l|}{ Sheep meat } \\
\hline 47 & \multicolumn{9}{|l|}{ Turkey } \\
\hline 48 & \multicolumn{9}{|l|}{ Ducks } \\
\hline 49 & \multicolumn{9}{|l|}{ Bush/game meat } \\
\hline 50 & Fish dried (Utaka) & & & & & & & & \\
\hline 51 & Fish (Fresh) & & & & & & & & \\
\hline 52 & Fish (Frozen) & & & & & & & & \\
\hline 53 & Tinned fish & & & & & & & & \\
\hline $\mathbf{E}$ & $\begin{array}{l}\text { Dairy products and } \\
\text { Eggs }\end{array}$ & & & & & & & & \\
\hline 54 & Eggs & & & & & & & & \\
\hline 55 & Milk & & & & & & & & \\
\hline 56 & Butter & & & & & & & & \\
\hline 57 & Cheese & & & & & & & & \\
\hline 58 & Yoghurt & & & & & & & & \\
\hline 59 & Other dairy products & & & & & & & & \\
\hline $\begin{array}{l}\text { Cod } \\
\text { 1. Te } \\
\text { 2. } \mathrm{Tr} \\
\text { 3. Sl } \\
\text { 4. Pi } \\
\text { 5. Cc } \\
\text { 6. Cl } \\
\text { 7. M } \\
\text { 8. Li } \\
\text { 9. Gi }\end{array}$ & $\begin{array}{l}\text { s A } \\
\text { aspoon } \\
\text { blespoon } \\
\text { ce of bread } \\
\text { ce } \\
\text { ffee/Tea cup } \\
\text { p (Standard) } \\
\text { lliliter } \\
\text { er } \\
\text { am }\end{array}$ & & $\begin{array}{l}\text { les A } \\
\text { Kilogram } \\
\text { Number } \\
\text { Lump of Nshima } \\
\text { Regular (Small) Chip } \\
\text { Pizza box (Small) } \\
\text { Cone (Ice cream) } \\
\text { Loaf of Bread } \\
\text { Packet (Sugar,...etc) } \\
\text { Heap } \\
\text { Bunch } \\
\text { Meda } \\
\text { Bp } \\
\text { Bottle/can } \\
\text { Crate }\end{array}$ & & & $\begin{array}{l}\text { B } \\
\text { erstore/Hyper s } \\
\text { ermarket } \\
\text { ivenience store } \\
\text { cery store } \\
\text { (Soweto) mark } \\
\text { ditional/Compo } \\
\text { ghborhood kios } \\
\text { idside market } \\
\text { ekly market } \\
\text { st food restaura } \\
\text { sual/Ordinary r } \\
\text { vn production } \\
\text { ft/Free food } \\
\text { hers, specify... }\end{array}$ & $\begin{array}{l}\text { t } \\
\text { nd market } \\
\text { s }\end{array}$ & 0. Almos & $\begin{array}{r}\text { Codes C } \\
\text { it the same in a year } \\
\text { 1. January } \\
\text { 2. February } \\
\text { 3. March } \\
\text { 4. April } \\
\text { 5. May } \\
\text { 6. June } \\
\text { 7. July } \\
\text { 8. August } \\
\text { 9. September } \\
\text { 10. October } \\
\text { 11. November } \\
\text { 12. December } \\
\text { 13. Not applicable }\end{array}$ \\
\hline
\end{tabular}


Here, wife and/or the person involved in purchases should be the principal respondent/s

Notes: Please include all foods consumed by all household members.

\begin{tabular}{|c|c|c|c|c|c|c|c|c|}
\hline \multirow[b]{2}{*}{ 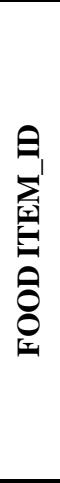 } & \multirow[b]{2}{*}{ FOOD ITEM } & \multirow[b]{2}{*}{ 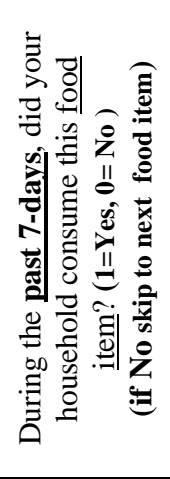 } & \multicolumn{6}{|c|}{ Food consumption expenditure in the past 7-days } \\
\hline & & & $\begin{array}{c}\text { How } \\
\text { much in } \\
\text { total was } \\
\text { consumed } \\
\text { by the HH } \\
\text { during last } \\
7 \text { days? } \\
\text { (Qty) }\end{array}$ & 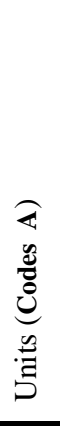 & $\begin{array}{c}\text { Average } \\
\text { price per } \\
\text { unit } \\
(\mathbf{Z M K})\end{array}$ & $\begin{array}{l}\text { Total cost of } \\
\text { food item } \\
\text { (ZMK) }\end{array}$ & $\begin{array}{c}\text { Where exactly } \\
\text { did your } \\
\text { household } \\
\text { purchase or } \\
\text { source this } \\
\text { food item? } \\
\text { ( Codes B) }\end{array}$ & $\begin{array}{l}\text { Which } \\
\text { month is this } \\
\text { food item } \\
\text { cheapest/ } \\
\text { plenty? } \\
\text { (Codes C) }\end{array}$ \\
\hline 1 & 2 & 3 & 4 & 5 & 6 & 7 & 8 & 9 \\
\hline $\mathrm{F}$ & $\begin{array}{l}\text { Vegetables and } \\
\text { Mushroom }\end{array}$ & & & & & & & \\
\hline 60 & Frozen vegetables & & & & & & & \\
\hline 61 & Tinned vegetables & & & & & & & \\
\hline 62 & Tomatoes & & & & & & & \\
\hline 63 & Tomatoes source & & & & & & & \\
\hline 64 & Onions & & & & & & & \\
\hline 65 & Rape/Mustard/Chinese & & & & & & & \\
\hline 66 & Cabbage & & & & & & & \\
\hline 67 & Lettuce & & & & & & & \\
\hline 68 & Bean leaves & & & & & & & \\
\hline 69 & Cassava leaves & & & & & & & \\
\hline 70 & Cowpea leaves & & & & & & & \\
\hline 71 & Pumpkin leaves & & & & & & & \\
\hline 72 & Blackjack & & & & & & & \\
\hline 73 & Okra & & & & & & & \\
\hline 74 & Carrot & & & & & & & \\
\hline 75 & Pepper & & & & & & & \\
\hline 76 & Garlic & & & & & & & \\
\hline 77 & Egg plant & & & & & & & \\
\hline 78 & Cucumber & & & & & & & \\
\hline 79 & Mixed salad & & & & & & & \\
\hline 80 & $\begin{array}{l}\text { Other vegetables } \\
\text { (Specify) }\end{array}$ & & & & & & & \\
\hline 81 & Mushroom (Cultivated) & & & & & & & \\
\hline 82 & Mushroom (Wild) & & & & & & & \\
\hline $\mathbf{G}$ & Fruits (1/2) & & & & & & & \\
\hline 83 & Mixed fruits & & & & & & & \\
\hline 84 & Tinned fruits & & & & & & & \\
\hline 85 & Frozen fruits & & & & & & & \\
\hline 86 & Apples & & & & & & & \\
\hline 87 & Avocadoes & & & & & & & \\
\hline 88 & Banana (Ripe) & & & & & & & \\
\hline 89 & Guavas & & & & & & & \\
\hline
\end{tabular}

\begin{tabular}{|c|c|c|c|}
\hline $\begin{array}{l}\text { Codes A } \\
\text { 1. Teaspoon } \\
\text { 2. Tablespoon } \\
\text { 3. Slice of bread } \\
\text { 4. Piece } \\
\text { 5. Coffee/Tea cup } \\
\text { 6. Cup (Standard) } \\
\text { 7. Milliliter } \\
\text { 8. Liter } \\
\text { 9. Gram }\end{array}$ & $\begin{array}{l}\text { Codes A } \\
\text { 10. Kilogram } \\
\text { 11. Number } \\
\text { 12. Lump of Nshima } \\
\text { 13. Regular (Small) Chips } \\
\text { 14. Pizza box (Small) } \\
\text { 15. Cone (Ice cream) } \\
\text { 16. Loaf of Bread } \\
\text { 17. Packet (Sugar,...etc) } \\
\text { 18. Heap } \\
\text { 19. Bunch } \\
\text { 20. Meda } \\
\text { 21. Bp } \\
\text { 22. Bottle/can } \\
\text { 23. Crate }\end{array}$ & $\begin{array}{l}\text { Codes B } \\
\text { 1. Superstore/Hyper store } \\
\text { 2. Supermarket } \\
\text { 3. Convenience store } \\
\text { 4. Grocery store } \\
\text { 5. Wet (Soweto) market } \\
\text { 6. Traditional/Compound market } \\
\text { 7. Neighborhood kiosks } \\
\text { 8. Roadside market } \\
\text { 9. Weekly market } \\
\text { 10. Fast food restaurant } \\
\text { 11. Casual/Ordinary restaurant } \\
\text { 12. Own production } \\
\text { 13. Gift/Free food } \\
\text { 14. Others, specify...... }\end{array}$ & $\begin{array}{r}\text { Codes C } \\
\text { 0. Almost the same in a year } \\
\text { 1. January } \\
\text { 2. February } \\
\text { 3. March } \\
\text { 4. April } \\
\text { 5. May } \\
\text { 6. June } \\
\text { 7. July } \\
\text { 8. August } \\
\text { 9. September } \\
\text { 10. October } \\
\text { 11. November } \\
\text { 12. December } \\
\text { 13. Not applicable }\end{array}$ \\
\hline
\end{tabular}


Here, wife and/or the person involved in purchases should be the principal respondent/s

Notes: Please include all foods consumed by all household members.

\begin{tabular}{|c|c|c|c|c|c|c|c|c|c|}
\hline \multirow[b]{2}{*}{ 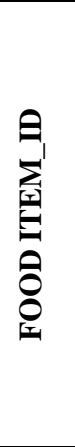 } & \multirow{2}{*}{\multicolumn{2}{|c|}{ FOOD ITEM }} & \multirow[b]{2}{*}{ 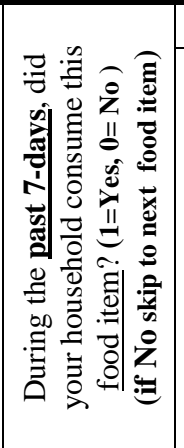 } & \multicolumn{6}{|c|}{ Food consumption expenditure in the past 7-days } \\
\hline & & & & $\begin{array}{c}\text { How } \\
\text { much in } \\
\text { total was } \\
\text { consumed } \\
\text { by the HH } \\
\text { during last } \\
7 \text { days? } \\
\text { (Qty) }\end{array}$ & 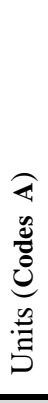 & $\begin{array}{c}\text { Average } \\
\text { price per } \\
\text { unit } \\
(\mathbf{Z M K}) \\
\end{array}$ & $\begin{array}{l}\text { Total cost of } \\
\text { food item } \\
(\mathbf{Z M K})\end{array}$ & $\begin{array}{c}\text { Where exactly } \\
\text { did your } \\
\text { household } \\
\text { purchase or } \\
\text { source this } \\
\text { food item? } \\
\text { ( Codes B) }\end{array}$ & $\begin{array}{l}\text { Which } \\
\text { month is this } \\
\text { food item } \\
\text { cheapest/ } \\
\text { plenty? } \\
\text { (Codes C) }\end{array}$ \\
\hline 1 & \multicolumn{2}{|l|}{2} & 3 & 4 & 5 & 6 & 7 & 8 & 9 \\
\hline G & \multicolumn{2}{|l|}{ Fruits (2/2) } & & & & & & & \\
\hline 90 & \multicolumn{2}{|l|}{ Oranges/Tangerines } & & & & & & & \\
\hline 91 & \multicolumn{2}{|l|}{ Pawpaws } & & & & & & & \\
\hline 92 & \multicolumn{2}{|l|}{ Pineapples } & & & & & & & \\
\hline 93 & \multicolumn{2}{|l|}{ Mangoes } & & & & & & & \\
\hline 94 & \multicolumn{2}{|l|}{ Watermelons } & & & & & & & \\
\hline 95 & \multicolumn{2}{|l|}{ Other fruits (Specify) } & & & & & & & \\
\hline $\mathbf{H}$ & \multicolumn{2}{|c|}{$\begin{array}{l}\text { Food away from home } \\
\text { (take/eat aways etc) }\end{array}$} & & & & & & & \\
\hline 96 & \multicolumn{2}{|l|}{ Chips } & & & & & & & \\
\hline 97 & \multicolumn{2}{|l|}{ Nshima } & & & & & & & \\
\hline 98 & \multicolumn{2}{|l|}{ Rice } & & & & & & & \\
\hline 99 & \multicolumn{2}{|l|}{ Prepared meat } & & & & & & & \\
\hline 100 & \multicolumn{2}{|l|}{ Prepared fish } & & & & & & & \\
\hline 101 & \multicolumn{2}{|l|}{ Prepared vegetables } & & & & & & & \\
\hline 102 & \multicolumn{2}{|l|}{ Mandazi } & & & & & & & \\
\hline 103 & \multicolumn{2}{|l|}{ Samosa } & & & & & & & \\
\hline 104 & \multicolumn{2}{|l|}{ Prepared pasta } & & & & & & & \\
\hline 105 & \multicolumn{2}{|l|}{ Prepared sausage } & & & & & & & \\
\hline 106 & \multicolumn{2}{|l|}{ Pizza (Fresh/Frozen) } & & & & & & & \\
\hline I & \multicolumn{2}{|l|}{$\begin{array}{l}\text { Partially prepared or } \\
\text { ready food (PPF) }\end{array}$} & & & & & & & \\
\hline 107 & \multicolumn{2}{|l|}{ Crips } & & & & & & & \\
\hline 108 & Puffed salted corn chi & hips & & & & & & & \\
\hline 109 & Salted nuts & & & & & & & & \\
\hline 110 & Popcorn & & & & & & & & \\
\hline 111 & Other ready meals & & & & & & & & \\
\hline 112 & Instant noodles & & & & & & & & \\
\hline 113 & Other PPF (Specify) & & & & & & & & \\
\hline $\mathbf{J}$ & Indigenous food (IF) & & & & & & & & \\
\hline 114 & Caterpillars/ants & & & & & & & & \\
\hline 115 & Masuku & & & & & & & & \\
\hline 116 & Masau & & & & & & & & \\
\hline 117 & Other IFs (Specify) & & & & & & & & \\
\hline $\begin{array}{l}\text { Codes } \\
\text { 1. Tea } \\
\text { 2. Tab } \\
\text { 3. Slic } \\
\text { 4. Piec } \\
\text { 5. Cof } \\
\text { 6. Cup } \\
\text { 7. Mil } \\
\text { 8. Lite } \\
\text { 9. Gra }\end{array}$ & $\begin{array}{l}\text { on } \\
\text { poon } \\
\text { f bread } \\
\text { /Tea cup } \\
\text { tandard) } \\
\text { ter }\end{array}$ & $\begin{array}{l}\text { Code } \\
\text { 10. K } \\
11 . \mathrm{N} \\
\text { 12. } \mathrm{L} \\
\text { 13. R } \\
\text { 14. P } \\
\text { 15. C } \\
\text { 16. L } \\
\text { 17. P } \\
\text { 18. } \mathrm{H} \\
\text { 19. B } \\
\text { 20. N } \\
\text { 21. B } \\
\text { 22. B } \\
\text { 23. C }\end{array}$ & $\begin{array}{l}\text { A } \\
\text { logram } \\
\text { mber } \\
\text { mp of Nshima } \\
\text { gular (Small) Chips } \\
\text { za box (Small) } \\
\text { ne (Ice cream) } \\
\text { af of Bread } \\
\text { cket (Sugar,...etc) } \\
\text { ap } \\
\text { inch } \\
\text { eda } \\
\text { ttle/can } \\
\text { ate }\end{array}$ & & & $\begin{array}{l}\text { B } \\
\text { erstore/Hyper s } \\
\text { ermarket } \\
\text { venience store } \\
\text { cery store } \\
\text { (Soweto) mark } \\
\text { litional/Compo } \\
\text { hborhood kios } \\
\text { dside market } \\
\text { kly market } \\
\text { t food restaura } \\
\text { sual/Ordinary r } \\
\text { in production } \\
\text { t/Free food } \\
\text { ters, specify... }\end{array}$ & market & 0. Almos & $\begin{array}{r}\text { Codes } \mathbf{C} \\
\text { the same in a year } \\
\text { 1. January } \\
\text { 2. February } \\
\text { 3. March } \\
\text { 4. April } \\
\text { 5. May } \\
\text { 6. June } \\
7 . \text { July } \\
\text { 8. August } \\
\text { 9. September } \\
\text { 10. October } \\
\text { 11. November } \\
\text { 12. December } \\
\text { 13. Not applicable }\end{array}$ \\
\hline
\end{tabular}


Here, wife and/or the person involved in purchases should be the principal respondent/s

Notes: Please include all foods consumed by all household members.

\begin{tabular}{|c|c|c|c|c|c|c|c|c|c|}
\hline \multirow[b]{2}{*}{ 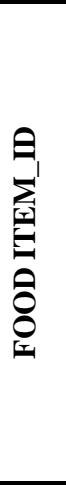 } & \multirow{2}{*}{\multicolumn{2}{|c|}{ FOOD ITEM }} & \multirow[b]{2}{*}{ 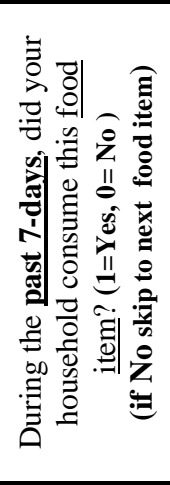 } & \multicolumn{6}{|c|}{ Food consumption expenditure in the past 7-days } \\
\hline & & & & $\begin{array}{c}\text { How } \\
\text { much in } \\
\text { total was } \\
\text { consumed } \\
\text { by the HH } \\
\text { during last } \\
7 \text { days? } \\
\text { (Qty) }\end{array}$ & 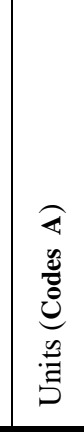 & $\begin{array}{c}\text { Average } \\
\text { price per } \\
\text { unit } \\
(\mathbf{Z M K})\end{array}$ & $\begin{array}{l}\text { Total cost of } \\
\text { food item } \\
(\mathbf{Z M K})\end{array}$ & $\begin{array}{c}\text { Where } \\
\text { exactly did } \\
\text { your } \\
\text { household } \\
\text { purchase or } \\
\text { source this } \\
\text { food item? } \\
\text { ( Codes B) }\end{array}$ & $\begin{array}{l}\text { Which } \\
\text { month is } \\
\text { this food } \\
\text { item } \\
\text { cheapest/ } \\
\text { plenty? } \\
\text { (Codes C) }\end{array}$ \\
\hline 1 & \multicolumn{2}{|l|}{2} & 3 & 4 & 5 & 6 & 7 & 8 & 9 \\
\hline $\mathbf{K}$ & \multicolumn{9}{|c|}{$\begin{array}{l}\text { Fats, Oils, Sweeteners, } \\
\text { Snacks and Others }\end{array}$} \\
\hline 118 & \multicolumn{9}{|c|}{ Cooking oil/fat } \\
\hline 119 & \multicolumn{9}{|l|}{ Groundnut (Flour) } \\
\hline 120 & \multicolumn{9}{|l|}{ Coconut oil } \\
\hline 121 & \multicolumn{9}{|l|}{ Margarine/Butter } \\
\hline 122 & \multicolumn{9}{|l|}{ Jam } \\
\hline 123 & \multicolumn{9}{|l|}{ Salt } \\
\hline 124 & \multicolumn{9}{|l|}{ Sugar } \\
\hline 125 & \multicolumn{9}{|l|}{ Biscuits/Cookies } \\
\hline 126 & \multicolumn{9}{|l|}{ Cake } \\
\hline 127 & \multicolumn{9}{|l|}{ Chocolate } \\
\hline 128 & \multicolumn{9}{|l|}{ Curry } \\
\hline 129 & \multicolumn{9}{|l|}{ Ginger } \\
\hline 130 & \multicolumn{9}{|l|}{ Cashew nuts } \\
\hline 131 & \multicolumn{9}{|l|}{ Macadamia nuts } \\
\hline 132 & \multicolumn{9}{|l|}{ Popcorn } \\
\hline 133 & \multicolumn{9}{|c|}{ Sugarcane } \\
\hline $\mathbf{L}$ & \multicolumn{9}{|c|}{ Beverages and Drinks } \\
\hline 134 & \multicolumn{9}{|c|}{ Tea (Leaves) } \\
\hline 135 & \multicolumn{9}{|l|}{ Coffee (Powder) } \\
\hline 136 & \multicolumn{9}{|l|}{ Ricoffy (Powder) } \\
\hline 137 & \multicolumn{9}{|c|}{ Soft drinks (Cococola, } \\
\hline 138 & \multicolumn{9}{|c|}{$\begin{array}{l}\text { Juices (Apple, } \\
\text { Orange etc) }\end{array}$} \\
\hline 139 & Bottled/Clear beer & & & & & & & & \\
\hline 140 & Opaque beer ( $\mathrm{Chil}$ & ku) & & & & & & & \\
\hline 141 & Local beer & & & & & & & & \\
\hline 142 & Wine & & & & & & & & \\
\hline 143 & Drinking water & & & & & & & & \\
\hline $\begin{array}{l}\text { Codes } \\
\text { 1. Tea } \\
\text { 2. Tab } \\
\text { 3. Slic } \\
\text { 4. Piec } \\
\text { 5. Cof } \\
\text { 6. Cup } \\
\text { 7. Mil } \\
\text { 8. Lite } \\
\text { 9. Gra }\end{array}$ & $\begin{array}{l}\text { oon } \\
\text { spoon } \\
\text { of bread } \\
\text { e/Tea cup } \\
\text { Standard) } \\
\text { iter }\end{array}$ & $\begin{array}{l}\text { Cod } \\
\text { 10. I } \\
11 . \text { I } \\
\text { 12. I } \\
\text { 13. I } \\
\text { 14. I } \\
\text { 15. . } \\
\text { 16. I } \\
\text { 17. I } \\
\text { 18. I } \\
\text { 19. I } \\
20.1 \\
21 . \text { I } \\
22 . \text { I } \\
23 .\end{array}$ & $\begin{array}{l}\text { ram } \\
\text { er } \\
\text { of Nshima } \\
\text { ar (Small) Chips } \\
\text { box (Small) } \\
\text { (Ice cream) } \\
\text { of Bread } \\
\text { ( (Sugar,...etc) } \\
\\
\text { /can }\end{array}$ & & $\begin{array}{l}\text { Codes I } \\
\text { 1. Super } \\
\text { 2. Super } \\
\text { 3. Conv } \\
\text { 4. Groce } \\
\text { 5. Wet ( } \\
\text { 6. Tradi } \\
\text { 7. Neigh } \\
\text { 8. Road } \\
\text { 9. Week } \\
\text { 10. Fast } \\
\text { 11. Cast } \\
\text { 12. Own } \\
\text { 13. Gift } \\
\text { 14. Othe }\end{array}$ & $\begin{array}{l}\text { ore/Hyper sto } \\
\text { arket } \\
\text { ience store } \\
\text { y store } \\
\text { oweto) marke } \\
\text { onal/Compou } \\
\text { orhood kiosk } \\
\text { de market } \\
\text { market } \\
\text { ood restaurant } \\
\text { 1/Ordinary res } \\
\text { roduction } \\
\text { ree food } \\
\text { s, specify..... }\end{array}$ & market & 0. Alm & $\begin{array}{r}\text { Codes C } \\
\text { the same in a year } \\
\text { 1. January } \\
\text { 2. February } \\
\text { 3. March } \\
\text { 4. April } \\
\text { 5. May } \\
\text { 6. June } \\
\text { 7. July } \\
\text { 8. August } \\
\text { 9. September } \\
\text { 10. October } \\
\text { 11. November } \\
\text { 12. December } \\
\text { 13. Not applicable }\end{array}$ \\
\hline
\end{tabular}


PART 3: ACCESS TO FOOD OUTLETS, HEALTH FACILTIES AND SHOPPING BEHAVIOUR

\begin{tabular}{|c|c|c|c|c|c|c|c|c|c|c|c|c|}
\hline 罯 & $\begin{array}{c}\text { FACILITY } \\
\text { Did you use this facility during } \\
\text { the last } 30 \text { days? } \\
(\mathbf{1 = Y e s , ~} \mathbf{0 =} \mathbf{N o}) \\
\text { (if No skip to next facility) }\end{array}$ & 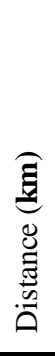 & $\begin{array}{l}\text { What is } \\
\text { the } \\
\text { common } \\
\text { mode of } \\
\text { transport } \\
\text { you use to } \\
\text { the } \\
\text { facility? } \\
\text { (Codes A) }\end{array}$ & $\begin{array}{l}\text { Travelling } \\
\text { time to } \\
\text { this } \\
\text { facility } \\
\text { (one way) } \\
\text { (min) }\end{array}$ & $\begin{array}{l}\text { Travelling } \\
\text { cost to } \\
\text { this } \\
\text { facility } \\
\text { (one way) } \\
\text { (ZMK) }\end{array}$ & $\begin{array}{l}\text { Is the most } \\
\text { food bought } \\
\text { on the way } \\
\text { to home } \\
\text { from work? } \\
\text { (1=Yes, } \\
\mathbf{0 = N o})\end{array}$ & $\begin{array}{c}\text { How } \\
\text { many } \\
\text { times did } \\
\text { you buy } \\
\text { food from } \\
\text { this outlet } \\
\text { during the } \\
\text { last } 30 \\
\text { days? }\end{array}$ & $\begin{array}{l}\text { What are the } \\
\text { most important } \\
\text { reasons you } \\
\text { prefer } \\
\text { shopping/eating } \\
\text { at this outlet? } \\
\text { (Codes B) }\end{array}$ & $\begin{array}{c}\text { How do you } \\
\text { learn about } \\
\text { new/ } \\
\text { promotion on } \\
\text { food } \\
\text { products? } \\
\text { (Codes C) }\end{array}$ & $\begin{array}{l}\text { Which food } \\
\text { products } \\
\text { would you } \\
\text { consider are } \\
\text { cheapest from } \\
\text { this food } \\
\text { outlet? } \\
\text { (Codes D) }\end{array}$ & $\begin{array}{l}\text { For how } \\
\text { long have } \\
\text { you been } \\
\text { using this } \\
\text { facility? } \\
\text { (Years) }\end{array}$ & $\begin{array}{l}\text { Number of } \\
\text { facilities in or } \\
\text { closer to the } \\
\text { compound }\end{array}$ \\
\hline 1 & 2 & 3 & 4 & 5 & 6 & 7 & 8 & 9 & 10 & 11 & 12 & 13 \\
\hline A & Food Outlet & & & & & & & & & & & \\
\hline 301 & Superstore/Hyper store & & & & & & & & & & & \\
\hline 302 & Supermarket & & & & & & & & & & & \\
\hline 303 & Convenience store & & & & & & & & & & & \\
\hline 304 & Grocery store & & & & & & & & & & & \\
\hline 305 & Wet (Soweto) market & & & & & & & & & & & \\
\hline 306 & Traditional/Compound market & & & & & & & & & & & \\
\hline 307 & Neighborhood kiosks & & & & & & & & & & & \\
\hline 308 & Roadside market & & & & & & & & & & & \\
\hline 309 & Weekly market & & & & & & & & & & & \\
\hline 310 & Fast food restaurant & & & & & & & & & & & \\
\hline 311 & Casual/ordinary restaurant & & & & & & & & & & & \\
\hline B & Health Facility & & & & & & & & & & & \\
\hline 312 & Nearest clinic & & & & & & & & & & & \\
\hline 313 & Health center/Central hospital & & & & & & & & & & & \\
\hline
\end{tabular}

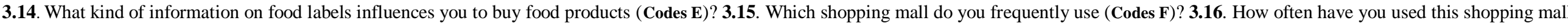
in the last 30 days?

\begin{tabular}{|c|c|c|}
\hline 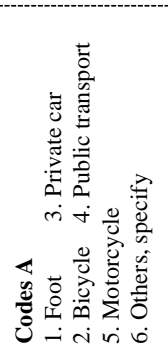 & $\begin{array}{l}\text { Codes B } \\
\text { 1. Lower prices } \\
\text { 2. Variety of food products } \\
\text { 3. Availability of food products } \\
\text { 4. Offer high quality food products } \\
\text { 5. Specialty of food products } \\
\text { 6. Closer to home } \\
\text { 7. Promotions/discounts } \\
\text { 8. Foods products under one roof } \\
\text { 9. Shopping atmosphere } \\
\text { 10. Social status } \\
\text { 11. Different packaging sizes }\end{array}$ & $\begin{array}{l}\text { 12.Variety of non-food products } \\
\text { 13. Time saving } \\
\text { 14. Higher perceived food safety } \\
\text { 15. Self service } \\
\text { 16. Long opening hours } \\
\text { 17. Customer service } \\
\text { 18. Others, specify }\end{array}$ \\
\hline
\end{tabular}

Codes C

1. TV adverts

2. Radio advert

4. Neighbors

5. Friends

6. Food retailer adverts

7. Medical adviser

8. Monthly promotion

9. Special offers
Codes D

1. Cereals
2. Roots \& tubers
3. Legumes

4. Meat \& Eggs

5. Milk products

6. Fruits

8. Bakery products

9. Oil products

129
Codes E

1. Expiry date
2. Serving size

3. Calories/Energy

$\begin{array}{ll}\text { 4. Total fat } & \text { 12. Iron } \\ \text { 13. ZABS laber }\end{array}$

5. Saturated fat 14. Brand name

6. Carbohydrates 15. List of ingredients

7. Added sugar 16. Date of manufacture

8. Fiber

9. Protein

10. Vitamins

11. Calcium
11. Vamins
Codes F

1. Arcades

1. Arcade
2. Cairo

3. Kabulonga

3. Kabulonga
4. Chawama

complex 16. Matero

6. Cosmopolitan 17. PHI

$\begin{array}{ll}\text { 7. Cross roads } & \text { 18. Changoz } \\ \text { 8. Down Town } & \text { 19. Chilenge }\end{array}$

$\begin{array}{ll}\text { 9. East Park 20. SOS } & \text { 21. Twin Palm }\end{array}$

$\begin{array}{ll}\text { 10. Embassy } & 22 \text {. Waterfalls }\end{array}$

$\begin{array}{ll}\text { 11. Garden city } & 23 \text {. Woodlands }\end{array}$ 


\section{PART 4: NON-FOOD EXPENDITURE (12 MONTHS RECALL)}

Here, wife and/or the person involved in purchases should be the principal respondent/s

Notes: Please include all non-food expenditure of the whole household (all members) and not only respondent.

\begin{tabular}{|c|c|c|c|}
\hline 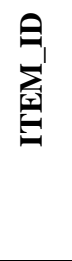 & EXPENSE ITEM OR SERVICE & $\begin{array}{l}\text { During the past 12-months } \\
\text { (Apr 17-Apr 18), did your } \\
\text { household spend on this } \\
\text { item or service? } \\
(1=\text { Yes, } 0=\text { No) } \\
\text { (if No skip to next item) }\end{array}$ & $\begin{array}{l}\text { How much did your household } \\
\text { spend on this item or service } \\
\text { during the last 12- months } \\
\text { (Apr 17-Apr 18) } \\
\text { (ZMK) }\end{array}$ \\
\hline 1 & 2 & 3 & 4 \\
\hline 401 & Soap/Washing products & & \\
\hline 402 & Personal care (Toothpaste, Nail, .........etc) & & \\
\hline 403 & House rent & & \\
\hline 404 & Electricity bills & & \\
\hline 405 & Candles & & \\
\hline 406 & Match boxes & & \\
\hline 407 & Fuel wood & & \\
\hline 408 & Charcoal & & \\
\hline 409 & Water bills & & \\
\hline 410 & Grain milling & & \\
\hline 411 & School fees & & \\
\hline 412 & School books and supplies & & \\
\hline 413 & School uniforms & & \\
\hline 414 & Newspapers, magazines, books, ....etc & & \\
\hline 415 & Fuel and engine oils for cars & & \\
\hline 416 & Car/Motorcycle insurance & & \\
\hline 417 & Public transport & & \\
\hline 418 & Other transport and travel expenses & & \\
\hline 419 & Air time (Mobile Phones, Landlines) & & \\
\hline 420 & DSTV/Gotv subscription & & \\
\hline 421 & Clothing & & \\
\hline 422 & Shoes & & \\
\hline 423 & Blankets & & \\
\hline 424 & Bed sheets & & \\
\hline 425 & Mosquito net & & \\
\hline 426 & Health care (Medical Care, Treatment, Insurance) & & \\
\hline 427 & Funeral payments (Costs, Policy, Life Insurance, ,...etc) & & \\
\hline 428 & Church contributions & & \\
\hline 429 & Dowry & & \\
\hline 430 & Contributions to associations/Cooperatives/Club & & \\
\hline 431 & Ceremony and other social activities & & \\
\hline 432 & Guard/security & & \\
\hline 433 & Kitchen utensils & & \\
\hline 434 & Furniture (Tables, Chairs, Beds, ... etc) & & \\
\hline 435 & Remittances paid & & \\
\hline 436 & Debt payments & & \\
\hline 437 & Home maintenance & & \\
\hline 438 & Payment for city rent in cash & & \\
\hline 439 & Purchase of agricultural inputs (Fertilizer, Seed,.....etc) & & \\
\hline 440 & Purchase of bicycle, motorcycle,,$\ldots .$. etc & & \\
\hline 441 & Purchase of cars & & \\
\hline 442 & House building/construction & & \\
\hline 443 & Other, specify............... & & \\
\hline
\end{tabular}




\section{PART 5: INCOME SOURCES, TRANSFERS AND HOUSEHOLD INCOME}

Notes: Please include all income sources including remittance for all members of the household if applicable and not only respondent.

\begin{tabular}{|c|c|c|c|c|c|}
\hline 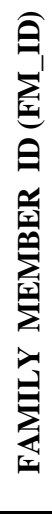 & $\begin{array}{c}\text { Name ( First Name, Last Name ) } \\
\text { of household member } \\
\text { (start with head of the household/respondent) }\end{array}$ & 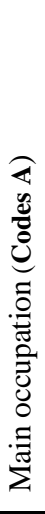 & $\begin{array}{c}\text { How } \\
\text { many months has } \\
\text { member working } \\
\text { in the last 12- } \\
\text { months } \\
\text { ( Apr 17-Apr 18) } \\
\text { (No/12) }\end{array}$ & $\begin{array}{c}\text { Average } \\
\text { monthly income } \\
\text { (ZMK/Month) }\end{array}$ & $\begin{array}{l}\text { Annual income } \\
\text { (ZMK) }\end{array}$ \\
\hline 1 & 2 & 3 & 4 & 5 & $6=4 \times 5$ \\
\hline 01 & & & & & \\
\hline 02 & & & & & \\
\hline 03 & & & & & \\
\hline 04 & & & & & \\
\hline 05 & & & & & \\
\hline 06 & & & & & \\
\hline 07 & & & & & \\
\hline 08 & & & & & \\
\hline 09 & & & & & \\
\hline 10 & & & & & \\
\hline
\end{tabular}

\begin{tabular}{|c|c|c|}
\hline Codes A & & \\
\hline 0. None & 4. Farming (Crop + Livestock) & 8. Non-school child \\
\hline 1. White- collar worker (Office job) & 5. Renting out houses & 9. Household chores. \\
\hline 2. Blue-collar worker (Manual labor) & 6. Casual laborer & 10. Pension fund. \\
\hline 3. Self-employed & 7. School/College child & 11. Others, specify... \\
\hline
\end{tabular}

5.7 Did your household receive money from relatives in the past six months $($ since October, 2017)? $(\mathbf{1}=$ Yes, $\mathbf{0 =}$ No)

5.8 If yes in 5.7, how many months did your household receive the money in past six months (since October, 2017)? (No/6)

5.9 On average, how much did your household receive per month?

5.10 What was the annual income from transfers or cash in kind? (ZMK/month) (ZMK) 
Notes: Respondents should exclude permanently broken items

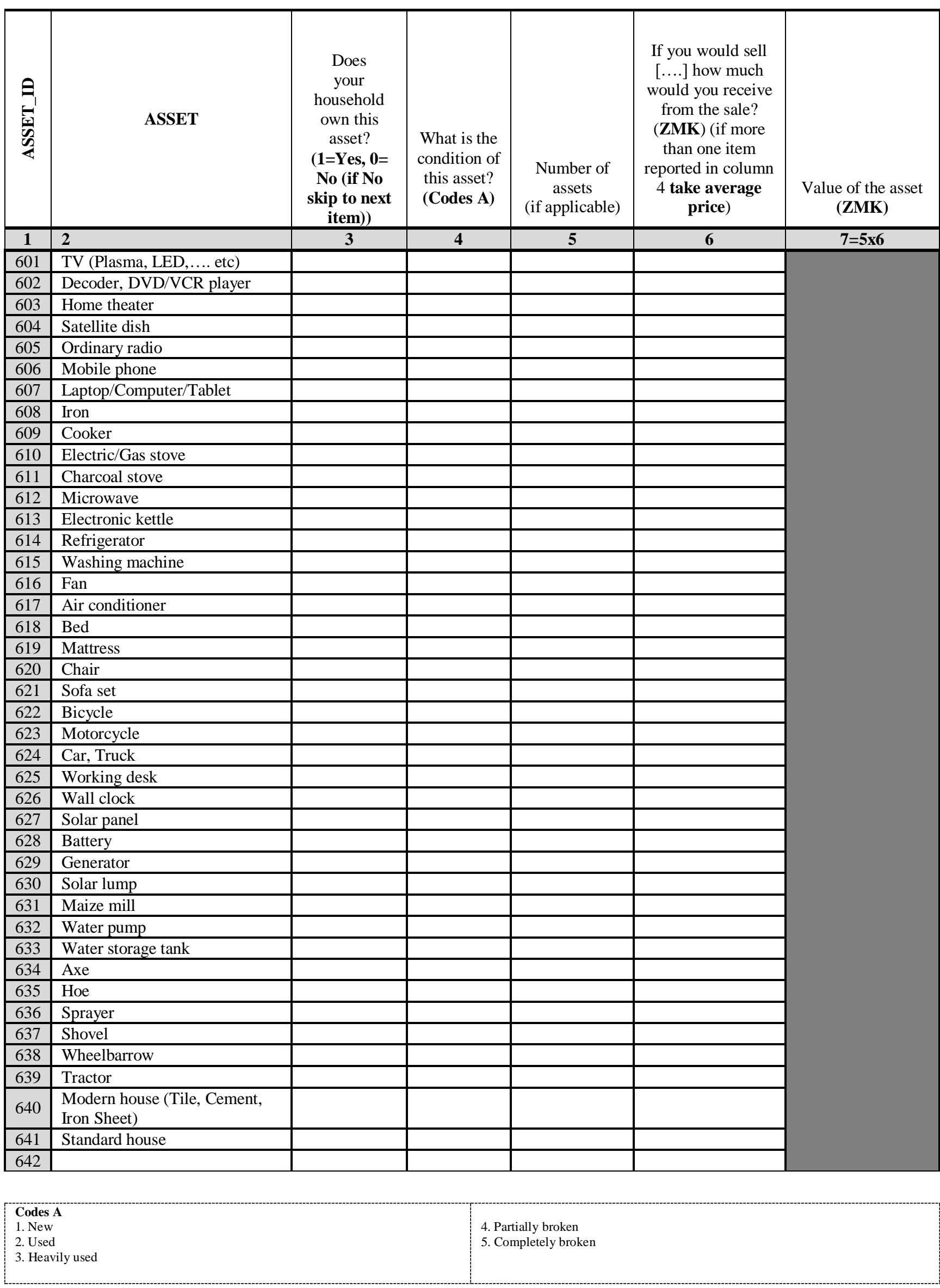


Notes: Interview at least FOUR individuals from the household including at least one child ( $<10$ years), one adolescent (10-18 years), and two adults (Male and Female) (>18 years) if applicable. For children ask their caretakers or parent (i.e., mothers).

\section{USE HARD COPY ON LISTING OF FAMILY MEMBER IDS AND FOOD DIARY BEFORE INTER VIEW IN THIS SECTION}

Probe for all food items consumed in the last 24 hours including food away from home, free food and food prepared at home. If the respondent had mixed dishes ask for ingredients that were used to prepare the food.

Family Member ID (from PART 1, Column 2): by this individual?

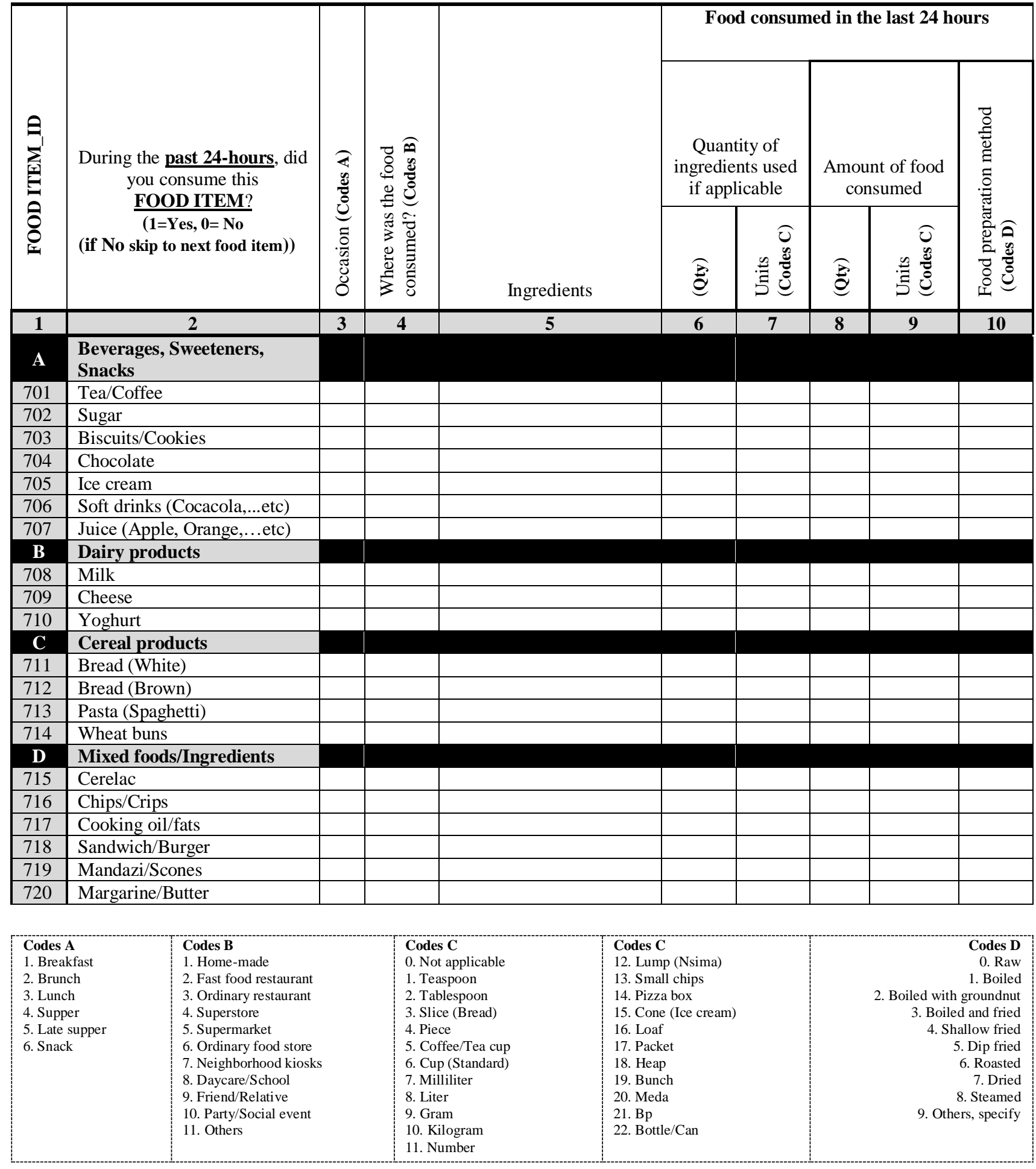




\begin{tabular}{|c|c|c|c|c|c|c|c|c|c|c|}
\hline \multirow{3}{*}{ 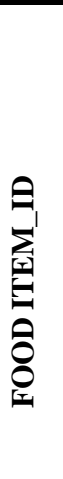 } & \multirow{3}{*}{\multicolumn{2}{|c|}{$\begin{array}{l}\text { During the past 24-hours, did } \\
\text { you consume this } \\
\text { FOOD ITEM? } \\
(1=\text { Yes, } 0=\text { No } \\
\text { (if No skip to next food item)) }\end{array}$}} & \multirow{3}{*}{ 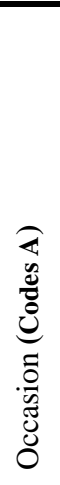 } & \multirow{3}{*}{ 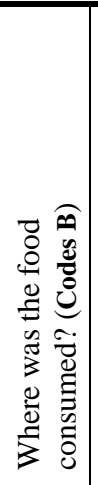 } & \multirow[b]{3}{*}{ Ingredients } & \multicolumn{5}{|c|}{ Food consumed in the last 24 hours } \\
\hline & & & & & & \multicolumn{2}{|c|}{$\begin{array}{l}\text { Quantity of } \\
\text { ingredients used } \\
\text { if applicable }\end{array}$} & \multicolumn{2}{|c|}{$\begin{array}{l}\text { Amount of food } \\
\text { consumed }\end{array}$} & \multirow{2}{*}{ 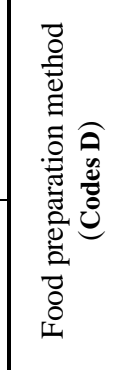 } \\
\hline & & & & & & ब્ڤ & 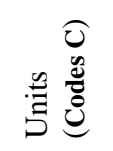 & $\widehat{E}$ & 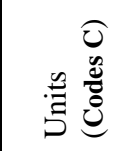 & \\
\hline 1 & & 2 & 3 & 4 & 5 & 6 & 7 & 8 & 9 & 10 \\
\hline 721 & \multicolumn{2}{|c|}{ Jam } & & & & & & & & \\
\hline 722 & \multicolumn{2}{|c|}{ Pizza (Fresh/Frozen) } & & & & & & & & \\
\hline 723 & \multicolumn{2}{|c|}{ Porridge } & & & & & & & & \\
\hline 724 & \multicolumn{2}{|c|}{ Samosa } & & & & & & & & \\
\hline 725 & \multicolumn{2}{|c|}{ Instant noodles } & & & & & & & & \\
\hline $\mathbf{E}$ & \multicolumn{2}{|c|}{ Roots and Tubers } & & & & & & & & \\
\hline 726 & \multicolumn{2}{|c|}{ Cassava } & & & & & & & & \\
\hline 727 & \multicolumn{2}{|c|}{ Pumpkins } & & & & & & & & \\
\hline 728 & \multicolumn{2}{|c|}{ Irish potatoes } & & & & & & & & \\
\hline 729 & \multicolumn{2}{|c|}{ Sweet potatoes (SP) } & & & & & & & & \\
\hline 730 & \multicolumn{2}{|c|}{ Orange fleshed (SP) } & & & & & & & & \\
\hline 731 & \multicolumn{2}{|c|}{ Yams } & & & & & & & & \\
\hline $\mathbf{F}$ & \multicolumn{2}{|c|}{ Cereal(s) products } & & & & & & & & \\
\hline 732 & Mai & & & & & & & & & \\
\hline 733 & Nsh & aize Mgaiwa) & & & & & & & & \\
\hline 734 & Nsh & iize Ufa Oyera) & & & & & & & & \\
\hline 735 & Nsh & ssava Flour) & & & & & & & & \\
\hline 736 & Rice & & & & & & & & & \\
\hline 737 & Sor & & & & & & & & & \\
\hline G & Leg & & & & & & & & & \\
\hline 738 & Con & ans & & & & & & & & \\
\hline 739 & Cov & y grain & & & & & & & & \\
\hline 740 & Gro & dry/flour & & & & & & & & \\
\hline 741 & Pige & dry grain & & & & & & & & \\
\hline 742 & Soy & & & & & & & & & \\
\hline 743 & Vel & s (Nzama) & & & & & & & & \\
\hline $\mathbf{H}$ & Me: & ish & & & & & & & & \\
\hline 744 & Bee & & & & & & & & & \\
\hline 745 & Chi & & & & & & & & & \\
\hline 746 & Duc & & & & & & & & & \\
\hline 747 & Sau & & & & & & & & & \\
\hline 748 & Sau & icken & & & & & & & & \\
\hline 749 & Sau & & & & & & & & & \\
\hline 750 & Egg & & & & & & & & & \\
\hline 751 & Fish & & & & & & & & & \\
\hline 752 & Goa & & & & & & & & & \\
\hline 753 & Pig & & & & & & & & & \\
\hline 754 & She & & & & & & & & & \\
\hline $\begin{array}{l}\text { Codes } \\
\text { 1. Bre } \\
\text { 2. Bru } \\
\text { 3. Lun } \\
\text { 4. Sup } \\
\text { 5. Late } \\
\text { 6. Sna }\end{array}$ & $\begin{array}{l}\text { fast } \\
\text { th } \\
\text { ar } \\
\text { upper }\end{array}$ & $\begin{array}{l}\text { Codes B } \\
\text { 1. Home-made } \\
\text { 2. Fast food restaurant } \\
\text { 3. Ordinary restaurant } \\
\text { 4. Superstore } \\
\text { 5. Supermarket } \\
\text { 6. Ordinary food store } \\
\text { 7. Neighborhood kiosks } \\
\text { 8. Daycare/School } \\
\text { 9. Friend/Relative } \\
\text { 10. Party/Social event } \\
\text { 11. Others }\end{array}$ & & & $\begin{array}{l}\text { des C } \\
\text { Not applicable } \\
\text { Teaspoon } \\
\text { Tablespoon } \\
\text { Slice (Bread) } \\
\text { Piece } \\
\text { Coffee/Tea cup } \\
\text { Cup (Standard) } \\
\text { Milliliter } \\
\text { Liter } \\
\text { Gram } \\
\text { Kilogram } \\
\text {. Number }\end{array}$ & $\begin{array}{l}\text { Codes C } \\
\text { 12. Lump (N } \\
\text { 13. Small ch } \\
\text { 14. Pizza bo } \\
\text { 15. Cone (Ic } \\
\text { 16. Loaf } \\
\text { 17. Packet } \\
\text { 18. Heap } \\
\text { 19. Bunch } \\
\text { 20. Meda } \\
\text { 21. Bp } \\
\text { 22. Bottle/C }\end{array}$ & & & $\begin{array}{r}\text { 2. Boiled wit } \\
\text { 3. Boil } \\
\text { 4. S } \\
\\
\text { 9. Ot }\end{array}$ & $\begin{array}{l}\text { Codes D } \\
0 . \text { Raw } \\
\text { 1. Boiled } \\
\text { groundnut } \\
\text { d and fried } \\
\text { allow fried } \\
\text { 5. Dip fried } \\
\text { 6. Roasted } \\
\text { 7. Dried } \\
\text { 8. Steamed } \\
\text { ers, specify }\end{array}$ \\
\hline
\end{tabular}




\begin{tabular}{|c|c|c|c|c|c|c|c|c|c|c|}
\hline \multirow{3}{*}{ 是 } & \multirow{3}{*}{\multicolumn{2}{|c|}{$\begin{array}{c}\text { During the past 24-hours, did } \\
\text { you consume this } \\
\text { FOOD ITEM? } \\
\begin{array}{c}(1=\text { Yes, } 0=\text { No } \\
\text { (if No skip to next food item)) }\end{array}\end{array}$}} & \multirow{3}{*}{ 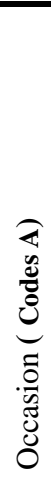 } & \multirow{3}{*}{ 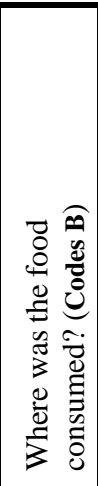 } & \multirow[b]{3}{*}{ Ingredients } & \multicolumn{5}{|c|}{ Food consumed in the last 24 hours } \\
\hline & & & & & & \multicolumn{2}{|c|}{$\begin{array}{l}\text { Quantity of } \\
\text { ingredients used } \\
\text { if applicable }\end{array}$} & \multicolumn{2}{|c|}{$\begin{array}{l}\text { Amount of food } \\
\text { consumed }\end{array}$} & \multirow{2}{*}{ 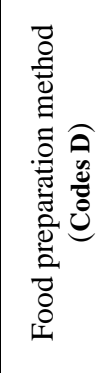 } \\
\hline & & & & & & $\widehat{\hat{\theta}}$ & 导 & $\widehat{\tilde{a}}$ & 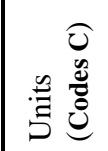 & \\
\hline 1 & \multicolumn{2}{|c|}{2} & 3 & 4 & 5 & 6 & 7 & 8 & 9 & 10 \\
\hline $\mathbf{I}$ & \multicolumn{2}{|c|}{ Fruits } & & & & & & & & \\
\hline 755 & \multicolumn{2}{|c|}{ Mixed fruits } & & & & & & & & \\
\hline 756 & \multicolumn{2}{|c|}{ Tinned fruits } & & & & & & & & \\
\hline 757 & \multicolumn{2}{|c|}{ Frozen fruits } & & & & & & & & \\
\hline 758 & \multicolumn{2}{|c|}{ Apples } & & & & & & & & \\
\hline 759 & \multicolumn{2}{|c|}{ Avocadoes } & & & & & & & & \\
\hline 760 & \multicolumn{2}{|c|}{ Bananas (Ripe) } & & & & & & & & \\
\hline 761 & \multicolumn{2}{|c|}{ Mangoes } & & & & & & & & \\
\hline 762 & \multicolumn{2}{|c|}{ Pawpaws } & & & & & & & & \\
\hline 763 & \multicolumn{2}{|c|}{ Pineapples } & & & & & & & & \\
\hline 764 & \multicolumn{2}{|c|}{ Oranges/Tangerines } & & & & & & & & \\
\hline 765 & \multicolumn{2}{|c|}{ Water melons } & & & & & & & & \\
\hline $\mathbf{J}$ & \multicolumn{2}{|c|}{ Vegetables and Mushroom } & & & & & & & & \\
\hline 766 & $\mathrm{Mix}$ & & & & & & & & & \\
\hline 767 & Tin & & & & & & & & & \\
\hline 768 & Fro & & & & & & & & & \\
\hline 769 & $\mathrm{Bac}$ & & & & & & & & & \\
\hline 770 & Bea & & & & & & & & & \\
\hline 771 & Bla & & & & & & & & & \\
\hline 772 & $\mathrm{Cab}$ & & & & & & & & & \\
\hline 773 & Cas & & & & & & & & & \\
\hline 774 & $\mathrm{Cov}$ & & & & & & & & & \\
\hline 775 & Let & & & & & & & & & \\
\hline 776 & Mo & & & & & & & & & \\
\hline 777 & Pun & & & & & & & & & \\
\hline 778 & Rap & & & & & & & & & \\
\hline 779 & Sw & ives & & & & & & & & \\
\hline 780 & $\mathrm{Okr}$ & & & & & & & & & \\
\hline 781 & Ton & & & & & & & & & \\
\hline 782 & Oni & & & & & & & & & \\
\hline 783 & Car & & & & & & & & & \\
\hline 784 & $\mathrm{Cuc}$ & & & & & & & & & \\
\hline 785 & Gre & & & & & & & & & \\
\hline 786 & Gre & & & & & & & & & \\
\hline 787 & $\mathrm{Mu}$ & & & & & & & & & \\
\hline $\mathbf{K}$ & Otl & & & & & & & & & \\
\hline 788 & Cat & & & & & & & & & \\
\hline $\begin{array}{l}\text { Codes } \\
\text { 1. Bre } \\
\text { 2. Bru } \\
\text { 3. Lun } \\
\text { 4. Sup } \\
\text { 5. Late } \\
\text { 6. Sna }\end{array}$ & $\begin{array}{l}\text { fast } \\
\text { h } \\
\text { upper }\end{array}$ & $\begin{array}{l}\text { Codes B } \\
\text { 1. Home-made } \\
\text { 2. Fast food restau } \\
\text { 3. Ordinary restau } \\
\text { 4. Superstore } \\
\text { 5. Supermarket } \\
\text { 6. Ordinary food s } \\
\text { 7. Neighborhood } 1 \\
\text { 8. Daycare/School } \\
\text { 9. Friend/Relative } \\
\text { 10. Party/Social e } \\
\text { 11. Others }\end{array}$ & $\begin{array}{l}\text { ant } \\
\text { ant } \\
\text { ore } \\
\text { iosks } \\
\text { ent }\end{array}$ & & $\begin{array}{l}\text { S C } \\
\text { t applicable } \\
\text { aspoon } \\
\text { olespoon } \\
\text { ce (Bread) } \\
\text { ce } \\
\text { ffee/Tea cup } \\
\text { p (Standard) } \\
\text { lliliter } \\
\text { er } \\
\text { am } \\
\text { ilogram } \\
\text { umber }\end{array}$ & $\begin{array}{l}\text { Codes } \\
\text { 12. Lur } \\
\text { 13. Sm } \\
\text { 14. Piz } \\
\text { 15. Cor } \\
\text { 16. Lor } \\
\text { 17. Pac } \\
\text { 18. He: } \\
\text { 19. Bur } \\
\text { 20. Me } \\
\text { 21. Bp } \\
\text { 22. Bot }\end{array}$ & $\begin{array}{l}\text { ima) } \\
\text { s } \\
\text { cream) } \\
\end{array}$ & & $\begin{array}{r} \\
\text { 2. Boiled } \\
\text { 3. } \mathrm{B} \\
4 \\
\\
\\
9 .\end{array}$ & $\begin{array}{r}\text { Codes D } \\
0 . \text { Raw } \\
\text { 1. Boiled } \\
\text { h groundnut } \\
\text { ed and fried } \\
\text { hallow fried } \\
\text { 5. Dip fried } \\
\text { 6. Roasted } \\
\text { 7. Dried } \\
\text { 8. Steamed } \\
\text { ers, specify }\end{array}$ \\
\hline
\end{tabular}


Here, wife and/or the person involved in purchases should be the principal respondent/s

Notes: Interview at least FOUR individuals SELECTED FOR PART 7.

\begin{tabular}{|c|c|c|c|c|c|c|c|c|c|c|c|c|c|c|c|}
\hline \multirow{2}{*}{ 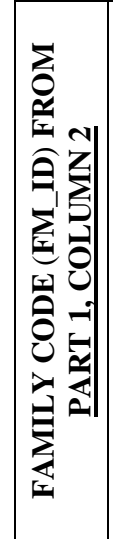 } & \multirow{2}{*}{\multicolumn{2}{|c|}{ Name of household member }} & \multirow{2}{*}{ 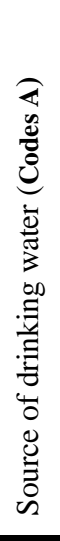 } & \multirow{2}{*}{ 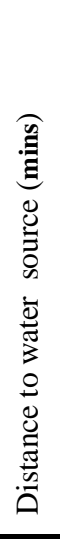 } & \multirow[b]{2}{*}{ 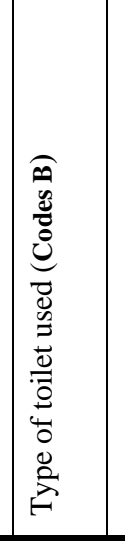 } & \multirow[b]{2}{*}{$\begin{array}{l}\text { Do you smoke? } \\
(\mathbf{1 = Y e s ,} \mathbf{0}=\mathbf{N o})\end{array}$} & \multirow[b]{2}{*}{$\begin{array}{c}\text { Has } \\
\text { anyone } \\
\text { suffered } \\
\text { from } \\
\text { chronic } \\
\text { illnesses? } \\
\text { (Codes C) }\end{array}$} & \multirow[b]{2}{*}{$\begin{array}{c}\text { Has } \\
\text { anyone suffered } \\
\text { from non-chronic } \\
\text { illnesses in the } \\
\text { last six months } \\
\text { (since Oct 17)? } \\
\text { (Codes D) }\end{array}$} & \multicolumn{2}{|c|}{$\begin{array}{c}\text { Annual } \\
\text { average } \\
\text { treatment cost } \\
\text { for } \\
(\mathbf{Z M K})\end{array}$} & \multicolumn{2}{|c|}{$\begin{array}{l}\text { Has your HH } \\
\text { experience any major } \\
\text { household shock in last } \\
\text { 12-months } \\
\text { (Apr 17-Apr 18)? }\end{array}$} & \multirow[b]{2}{*}{$\begin{array}{l}\text { How would } \\
\text { you rate the } \\
\text { overall } \\
\text { healthiness } \\
\text { of the diets } \\
\text { consumed } \\
\text { last } 30 \\
\text { days? } \\
\text { (Codes F) }\end{array}$} & \multirow[b]{2}{*}{$\begin{array}{l}\text { How would } \\
\text { you rate the } \\
\text { your } \\
\text { knowledge } \\
\text { about } \\
\text { nutrition } \\
\text { and health? } \\
\text { (Codes F) }\end{array}$} & \multirow[b]{2}{*}{$\begin{array}{c}\text { Are you on } \\
\text { special diets } \\
\text { due to } \\
\text { illness or } \\
\text { other } \\
\text { reasons? } \\
\text { (Codes G ) }\end{array}$} \\
\hline & & & & & & & & & 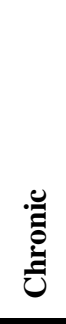 & 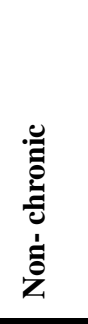 & \multicolumn{2}{|r|}{$\begin{array}{l}\text { Average } \\
\text { economic } \\
\text { loss to the } \\
\text { household } \\
\text { (ZMK) }\end{array}$} & & & \\
\hline 1 & \multicolumn{2}{|c|}{2} & 3 & 4 & 5 & 6 & 7 & 8 & 9 & 10 & 11 & 12 & 13 & 14 & 15 \\
\hline \multicolumn{16}{|l|}{01} \\
\hline \multicolumn{16}{|l|}{02} \\
\hline \multicolumn{16}{|l|}{03} \\
\hline \multicolumn{16}{|l|}{04} \\
\hline \multicolumn{16}{|l|}{05} \\
\hline \multicolumn{16}{|l|}{06} \\
\hline \multicolumn{16}{|l|}{07} \\
\hline $\begin{array}{l}\text { Codes A } \\
\text { 1. Piped } \\
\text { 2. Bottle } \\
\text { 3. Deep } \\
\text { 4. Deep } \\
\text { 5. Streal } \\
\text { 6. Boreh } \\
\text { 7. Other }\end{array}$ & $\begin{array}{l}\text { lap } \\
\text { water } \\
\text { ell protected } \\
\text { ell uncovered } \\
\text { le } \\
\text { specify }\end{array}$ & $\begin{array}{l}\text { Codes B } \\
\text { 1. Flash toilet pr } \\
\text { 2. Flash toilet sh } \\
\text { 3. Pit latrine priy } \\
\text { 4. Pit tatrine sha } \\
\text { 5. Other, specify }\end{array}$ & & & $\begin{array}{l}\text { odes C } \\
\text { No } \\
\text { Diabetes } \\
\text { Hypertension } \\
\text { Heart disease } \\
\text { Kwashiorkor } \\
\text { Cancel } \\
\text { Ananemia } \\
\text { Goiter } \\
\text { Bad teeth } \\
\text { Blindness }\end{array}$ & $\begin{array}{l}\text { 10. Ricketts } \\
\text { 11. Others, specify }\end{array}$ & $\begin{array}{l}\text { Codes D } \\
\text { 0. No } \\
\text { 1. Fever } \\
\text { 2. Diarrl } \\
\text { 3. Stom } \\
\text { 4. Vomi } \\
\text { 5. Flu/C } \\
\text { 6. Head } \\
\text { 7. Skin } 1 \\
\text { 8. Eye p } \\
\text { 9. Bad te }\end{array}$ & $\begin{array}{ll} & \text { 10.Tuberculosi } \\
\text { Malaria } & \text { 11. HIV/AIDS } \\
\text { a } & \text { 12. Pneumonia } \\
\text { th ache } & \text { 13. Typhoid } \\
\text { ng } & \text { 14. Intestinal } \\
\text { Id } & \text { 15. Others, spe } \\
\text { he } & \\
\text { oblems } & \\
\text { oblem } & \\
\text { th (Ache) } & \end{array}$ & $\begin{array}{l}\text { orms } \\
\text { ify... }\end{array}$ & $\begin{array}{l}\text { Codes } \\
\text { 0. No } \\
\text { 1. Deat } \\
\text { 2. Deat } \\
\text { 3. Theft } \\
\text { 4. Job 1 } \\
\text { 5. Acut } \\
\text { 6. Fire } \\
\text { 7. Natu } \\
\text { 8. Hous } \\
\text { 9. Conf }\end{array}$ & $\begin{array}{l}\text { of family meml } \\
\text { of close relativ } \\
\text { ss } \\
\text { illness } \\
\text { al calamities } \\
\text { damage }\end{array}$ & $\begin{array}{l}\text { Cod } \\
\text { 1. V } \\
\text { 2. G } \\
\text { 3. N } \\
\text { 4. A } \\
\text { 5. V } \\
\text { 6. N }\end{array}$ & $\begin{array}{l}\text { F } \\
\text { y good } \\
\text { d } \\
\text { mal } \\
\text { tle poor } \\
\text { y poor } \\
\text { sure }\end{array}$ & $\begin{array}{r}\text { 1. Food wi } \\
\text { 2. Food } \mathrm{w} \\
\text { 3. Foo } \\
\text { 5. A lot of } \mathrm{fi}_{\mathrm{i}} \\
7.0\end{array}$ & $\begin{array}{r}\text { Codes G } \\
0 . \text { No } \\
\text { less sugar or salt } \\
\text { th less oil and fats } \\
\text { rich in Vitamin A } \\
\text { Food rich in Iron } \\
\text { its and vegetables } \\
6 . \text { Child diet } \\
\text { hers, specify....... }\end{array}$ \\
\hline
\end{tabular}


Here, caretakers and/or parent (i.e. mother) should be the respondent/s on behalf of children

Notes: Interview at least FOUR individuals SELECTED IN PART 7. Take measurements for all individuals in Column 3 if applicable.

\begin{tabular}{|c|c|c|c|c|c|c|c|c|c|c|c|c|c|}
\hline \multirow[b]{2}{*}{$\begin{array}{c}\text { Sample } \\
\text { selected for } \\
\text { anthropometric } \\
\text { measurements } \\
\text { (Note: Do not take } \\
\text { measurements for pregnant } \\
\text { women) }\end{array}$} & \multirow{2}{*}{ 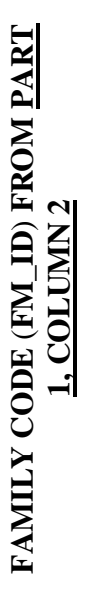 } & \multirow[b]{2}{*}{$\begin{array}{l}\text { Name of household } \\
\text { member }\end{array}$} & \multirow[b]{2}{*}{$\begin{array}{l}\text { How do you } \\
\text { mostly get to } \\
\text { work/school? } \\
\text { (Codes } \mathbf{A})\end{array}$} & \multicolumn{3}{|c|}{$\begin{array}{l}\text { What are the top } \underline{3} \\
\text { preferred leisure } \\
\frac{\text { activities? }}{(\text { Codes B })}\end{array}$} & \multirow[b]{2}{*}{$\begin{array}{l}\text { During the past } \\
\text { 7-days, how } \\
\text { many minutes } \\
\text { did you do } \\
\text { physical } \\
\text { activities during } \\
\text { leisure time? } \\
\text { (min/week) }\end{array}$} & \multirow{2}{*}{$\begin{array}{l}\text { How } \\
\text { would rate } \\
\text { your } \\
\text { physical } \\
\text { activities } \\
\text { during } \\
\text { work time? } \\
\text { (Codes C) }\end{array}$} & \multirow[b]{2}{*}{$\begin{array}{l}\text { What could } \\
\text { be the } \\
\text { reasons } \\
\text { you often } \\
\text { do physical } \\
\text { activities if } \\
\text { applicable? } \\
\text { (Codes D) }\end{array}$} & \multirow{2}{*}{$\begin{array}{l}\text { What could } \\
\text { be the } \\
\text { reasons you } \\
\text { rarely do } \\
\text { physical } \\
\text { activities if } \\
\text { applicable? } \\
\text { (Codes } \mathbf{E} \text { ) }\end{array}$} & \multicolumn{3}{|c|}{$\begin{array}{c}\text { Anthropometric measurements } \\
\text { (All members in column } 3 \text { without } \\
\text { heavy clothing and shoes if applicable) }\end{array}$} \\
\hline & & & & $1 \mathrm{st}$ & $2 \mathrm{nd}$ & $3 \mathrm{rd}$ & & & & & $\begin{array}{c}\text { Weight } \\
(\mathbf{k g})\end{array}$ & $\begin{array}{c}\text { Height } \\
(\mathbf{c m})\end{array}$ & $\begin{array}{l}\text { Waist } \\
(\mathbf{c m})\end{array}$ \\
\hline 1 & 2 & 3 & 4 & $5 \mathbf{a}$ & $5 \mathbf{b}$ & $5 c$ & 6 & 7 & 8 & 9 & 10 & 11 & 12 \\
\hline \multicolumn{14}{|l|}{ Male adult $(>18$ years $)$} \\
\hline \multicolumn{14}{|l|}{ Female adult ( $>18$ years) } \\
\hline \multicolumn{14}{|l|}{ Adolescent (10-18 years) } \\
\hline \multicolumn{14}{|l|}{ Children (6-9 years) } \\
\hline \multicolumn{14}{|l|}{ Children $(<6$ years $)$} \\
\hline $\begin{array}{l}\text { Codes A } \\
\text { 1. Public Bus/Minibus } \\
\text { 2. Private car } \\
\text { 3. Cycling } \\
\text { 4. Walking }\end{array}$ & $\begin{array}{l}\text { Codes B } \\
\text { 1. Watch } \\
\text { 2. Surfin } \\
\text { 3. Compu } \\
\text { 4. Housel } \\
\text { 5. GardeI } \\
\text { 6. Walki } \\
\text { 7. Basic } \\
\text { 8. Biking } \\
\text { 9. Basic } \\
\text { 10. Joggi } \\
\text { 11. Aerol }\end{array}$ & $\begin{array}{l}\text { g TV/Movies/Football } \\
\text { internet } \\
\text { er games } \\
\text { old chores } \\
\text { ng } \\
\text { for exercise } \\
\text { alking } \\
\text { or exercise } \\
\text { king } \\
\text { g/Running } \\
\text { atics }\end{array}$ & $\begin{array}{l}\text { 12. Weight lifting } \\
\text { 13. Football/Netball } \\
\text { 14. Volleyball } \\
\text { 15. Basket ball } \\
\text { 16. Tennis } \\
\text { 17. Drinking at home } \\
\text { 18. Drinking at public } \\
\text { 19. Reading (Newspap } \\
\text { 20. Meeting friends/So }\end{array}$ & $\begin{array}{l}\text { ace } \\
\text { Books) } \\
\text { al events }\end{array}$ & & & $\begin{array}{l}\text { Codes C- } \\
\text { 1. Hardly } \\
\text { 2. A bit } \\
\text { 3. Medium } \\
\text { 4. Strong } \\
\text { 5. Very strong }\end{array}$ & & $\begin{array}{l}\text { Hes D } \\
\text { Health advice by do } \\
\text { Beauty reasons (lose } \\
\text { Sport program for so } \\
\text { Weather reasons } \\
\text { Others, specify }\end{array}$ & $\begin{array}{l}\text { or/expert } \\
\text { weight) } \\
\text { ial event }\end{array}$ & & & $\begin{array}{l}\text { Codes E } \\
\text { 1. Lack of time } \\
\text { 2. Laziness } \\
\text { 3. Illness/Injury } \\
\text { 4. Bad weather } \\
\text { Gym is too costly } \\
\text { Thysical disability } \\
\text { There is no need } \\
\text { 8. Others, specify }\end{array}$ \\
\hline
\end{tabular}




\section{APPENDIX \\ Information for Study Participants}

\section{Dear participant:}

We would like to ask for your consent to participate in a research study to analyze possible effects of the modernization of food retailing on consumer food choices, diets, and nutrition in Zambia. The study is led by the University of Göttingen, Germany. The research depends on data to be collected from 475 households in Lusaka that are randomly selected for this purpose. Your household is one of these randomly selected households. The researchers involved in this study are Prof. Dr. Matin Qaim (Germany), Mr. Makaiko Khonje (Germany), and Ms. Patricia Sakala from the National Food and Nutrition Commission of Zambia.

Your participation in this research is voluntary. If you do not want to participate or would like to withdraw later on, there will be no negative consequences for you. You can drop out of the study at any time based on your preference. Please do not hesitate to ask additional questions if you need further details after reading (or listening to) the following information.

\section{Why are we doing this study?}

In Zambia, there is a change in the way people eat, drink and live their lives. In many cases, traditional diets are replaced by so called western-style diets with high intakes of highly processed food products and sugar, e.g., fast food, soft drinks, or other snacks. Concurrently, there is substantial growth in modern food retailers in Zambia, such as supermarkets, hypermarkets, and fast-food restaurants. Hence, we want to examine if people who buy or eat food from these modern food outlets make different food choices or have different diets and nutrition outcomes than people who primarily obtain their food from traditional markets. This research can help towards better understanding on how to improve nutrition and health in the context of modernizing food systems.

\section{What will be your part in our study?}

We want to explore how people eat and drink, and how their food choices and nutrition are possibly influenced by where they buy their food and other socioeconomic factors. Therefore, we will ask you and other family members questions about your food consumption habits and other aspects of your life and living conditions, including employment, education, and levels of physical activity. Furthermore, we need to measure your body height, body weight, and waist circumference. For these body measurements, you will have to stand on a height board and on a weighing scale without heavy clothing and shoes. We would like to collect such data from one male adult, one female adult, and one child or adolescent living in your household. The data collection does not involve any risk for you or your family members.

\section{What will be done with your data and measurements?}

All personal data collected will be seen only by the interviewers and the researchers directly involved in this study. Only these people will have access to the personal data. The data will be analyzed anonymously and the names of study participants will not be published or released to anyone not directly involved in the research study. We will treat the data with utmost confidentiality.

\section{What are advantages for you to participate in the study?}

If you participate in this study you will get to know your body size measurements. You will see if you are too thin or too thick for your height and age. The research team will advise you accordingly if you are overweight or obese. Further, at the end of the interview, we have a small gift as an appreciation for your participation in our study.

\section{What are your rights?}

You can withdraw from the study at any time. There are no negative consequences that you or your family will face if you decide not to participate or if you decide to drop out at any time. It is your free choice to participate in the measurements. If you withdraw, your data and information will be destroyed. Makaiko Khonje and his assistants will answer any questions you have regarding the study.

\section{What do you need to do if you decide to participate in the study?}

To participate in the study, you need to sign the "Declaration of Consent" form with your name and date. Parents and/or caretakers sign for children under the age of 18 years. If you have any questions, we will be happy to answer them. 


\section{Food Measurement Conversion Table}

Notes: To be used for PART 2 AND 7.

\begin{tabular}{|c|c|c|}
\hline MEASUREMENT_ID & Measurement & Metric conversion \\
\hline 1 & 2 & 3 \\
\hline 01 & 1 Teaspoon & $5 \mathrm{ml}$ \\
\hline 02 & 1 Tablespoon & $15 \mathrm{ml}$ \\
\hline 03 & 1 Slice of bread & $25 \mathrm{~g}$ \\
\hline 04 & 1 Piece (Tablespoon) & $20 \mathrm{~g}$ \\
\hline 05 & 1 Coffee/Tea cup & $150 \mathrm{ml}$ \\
\hline 06 & 1 Standard cup & $240 \mathrm{ml}$ \\
\hline 07 & 1 Milliliter & $1 \mathrm{ml}$ \\
\hline 08 & 1 Liter & $1000 \mathrm{ml}$ \\
\hline 09 & $1 \mathrm{Gram}$ & $1 \mathrm{~g}$ \\
\hline 10 & 1 Kilogram & $1000 \mathrm{~g}$ \\
\hline 11 & Number & \\
\hline 12 & 1 Lump of Nshima & $200 \mathrm{~g}$ \\
\hline 13 & 1 Regular (Small) Chips & $120 \mathrm{~g}$ \\
\hline 14 & 1 Pizza box (Small) & $300 \mathrm{~g}$ \\
\hline 15 & 1 Cone (Ice Cream) & $42 \mathrm{~g}$ \\
\hline 16 & 1 Loaf of bread & $500 \mathrm{~g}$ \\
\hline 17 & 1 Packet of Sugar/Salt & $1000 \mathrm{~g}$ \\
\hline 18 & 1 Heap & $250 \mathrm{ml}$ \\
\hline 19 & 1 Bunch & \\
\hline 20 & $1 \mathrm{Meda}$ & $5000 \mathrm{~g}$ \\
\hline 21 & $1 \mathrm{Bp}$ & \\
\hline 22 & 1 Bottle/Can & $330 \mathrm{ml}$ \\
\hline 23 & $1 / 2$ Crate & 10 bottles \\
\hline
\end{tabular}

\begin{tabular}{|l|c|c|}
\hline \multirow{2}{*}{ No. } & & \\
& Ingredient & Density (g/ml) \\
\hline $\mathbf{1}$ & & $\mathbf{3}$ \\
\hline 01 & $\mathbf{2}$ & 1.00 \\
\hline 02 & Water & 1.03 \\
\hline 03 & Milk & 0.92 \\
\hline 04 & Cooking oil & 1.20 \\
\hline 05 & Salt & 0.80 \\
\hline 06 & Sugar & 0.70 \\
\hline
\end{tabular}




\section{Food Diary Template for PART 7}

Household Identification Number (xxx):

Family Member Identification Number (FM_ID) (xx)

\begin{tabular}{|c|c|c|}
\hline Time/Meal & Food Item/Ingredients & Amount \\
\hline 1 & 2 & 3 \\
\hline \multirow{2}{*}{\multicolumn{3}{|c|}{ Break fast }} \\
\hline & & \\
\hline \multirow{2}{*}{\multicolumn{3}{|c|}{ Snack }} \\
\hline & & \\
\hline \multirow{2}{*}{\multicolumn{3}{|c|}{ Lunch }} \\
\hline & & \\
\hline \multirow{2}{*}{\multicolumn{3}{|c|}{ Snack }} \\
\hline & & \\
\hline \multicolumn{3}{|l|}{ Supper } \\
\hline & & \\
\hline Total & & \\
\hline
\end{tabular}

